\title{
2004 Annual Report
}

\section{Summer Research Institute Interfacial and Condensed Phase Chemical Physics}

Stephan Barlow, Director

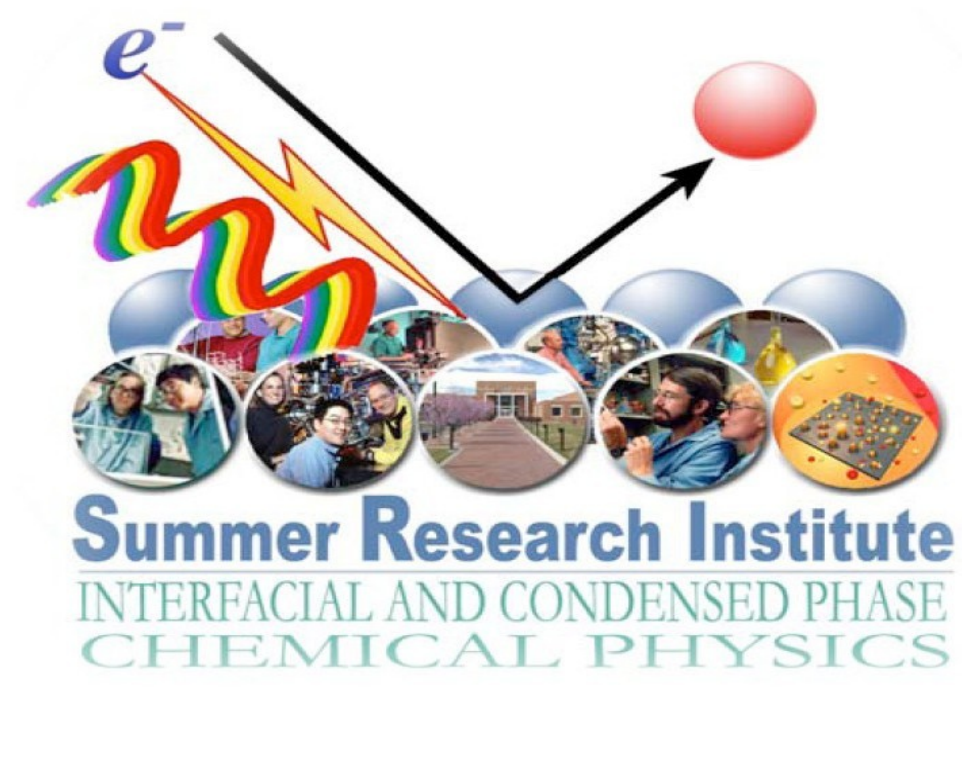




\title{
DISCLAIMER
}

This report was prepared as an account of work sponsored by an agency of the United States Government. Neither the United States Government nor any agency thereof, nor Battelle Memorial Institute, nor any of their employees, makes any warranty, express or implied, or assumes any legal liability or responsibility for the accuracy, completeness, or usefulness of any information, apparatus, product, or process disclosed, or represents that its use would not infringe privately owned rights. Reference herein to any specific commercial product, process, or service by trade name, trademark, manufacturer, or otherwise does not necessarily constitute or imply its endorsement, recommendation, or favoring by the United States Government or any agency thereof, or Battelle Memorial Institute. The views and opinions of authors expressed herein do not necessarily state or reflect those of the United States Government or any agency thereof.

\author{
PACIFIC NORTHWEST NATIONAL LABORATORY \\ operated by \\ BATTELLE \\ for the \\ UNITED STATES DEPARTMENT OF ENERGY
under Contract DE-AC06-76RL01830
}

Printed in the United States of America
Available to DOE and DOE contractors from the Office of Scientific and Technical Information,
P.O. Box 62, Oak Ridge, TN 37831-0062;
ph: (865) 576-8401
fax: (865) 576-5728
email: reports@adonis.osti.gov

\begin{abstract}
Available to the public from the National Technical Information Service, U.S. Department of Commerce, 5285 Port Royal Rd., Springfield, VA 22161 ph: (800) 553-6847 fax: (703) 605-6900

email: orders@ntis.fedworld.gov

online ordering: http://www.ntis.gov/ordering.htm
\end{abstract}

This document was printed on recycled paper. 


\section{Annual Report}

\section{Summer Research Institute Interfacial and Condensed Phase Chemical Physics}

October 2004

Prepared for

the U.S. Department of Energy

under Contract DE-AC06-76RL01830

Pacific Northwest National Laboratory

Richland, Washington 99352 


\section{Executive Summary}

Pacific Northwest National Laboratory (PNNL) hosted its first annual Summer Research Institute in Interfacial and Condensed Phase Chemical Physics from May through September 2004. During this period, fourteen PNNL scientists hosted sixteen young scientists from eleven universities. Of the sixteen participants, fourteen were graduate students, one was transitioning to graduate school, and one was a university faculty member. 
PNNL-14881

\section{Acknowledgments}

Even a modest-sized program such as this one could not happen without the assistance of many people. Dr. Steven D. Colson, Director of the Fundamental Science Directorate of Pacific Northwest National Laboratory (PNNL), led the initial organizing activities and has supported the institute since that time. Among the initial members of the organizing committee were Donald R. Baer, L. Rene Corrales, Stephan E. Barlow, and Roy E. Gephart from the PNNL scientific staff. Christine C. Montgomery, Administrator, played a key role in ensuring the institute ran smoothly. Cynthia A. Irwin, Operations Administrator for Chemical Structure and Dynamics, provided financial and budgetary support; and Jayme R. Crow provided invaluable administrative support.

We also acknowledge Rebecca J. Janosky and Lori L. Thompson of PNNL's Office of Fellowship Programs. They handled a myriad of details associated with fellowship appointments, including fellowship awards, agreements, and disbursement of stipends.

We also wish to acknowledge Amanda L. Kissire, Publications Design Group, Mark D. Bayless, Rich Interaction Environments, and Elaine C. Schneider, Graphics and Multimedia Design Group, respectively, for their expertise and timeliness in creating and developing the institute's website and application website and finalizing the institute's brochure.

Finally, we wish to acknowledge the U.S. Department of Energy's Office of Basic Energy Sciences, from whom we received the guidance to initiate the Summer Research Institute in Interfacial and Condensed Phase Chemical Physics and from whom support was ultimately derived.

The real stars of the Interfacial and Condensed Phase Chemical Physics Summer Research Institute were our participants. This document is largely devoted to them. 
PNNL-14881 


\section{Contents}

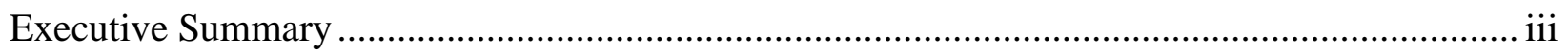

Acknowledgments.......................................................................................................... iv

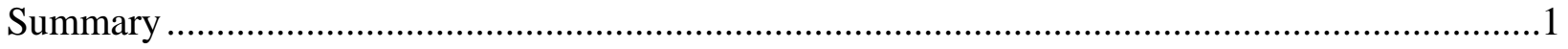

Participants and the Institute Structure ..................................................................................

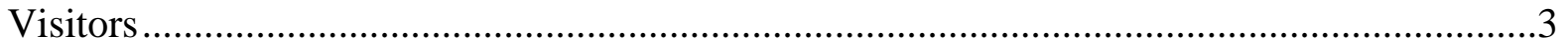

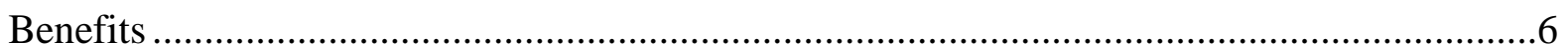

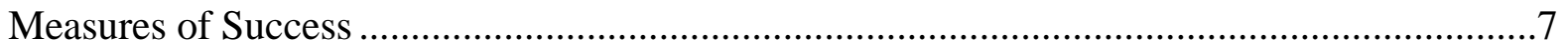

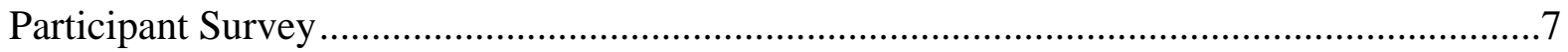

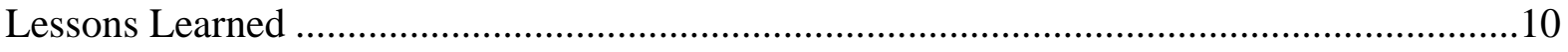

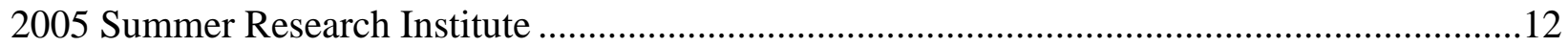

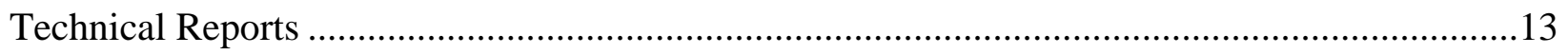

Alexandrova AN, A Boldyrev, and LS Wang, "Using GEGA Algorithm on the MPP2 Supercomputer" ..........................................................................................14

Alvarez J, RG Cooks, and J Laskin, "Soft-Landing of Peptide Ions onto Self-Assembled Monolayer Surfaces” ..................................................................................15

Bera D, S Seal, and DR Baer, "Growth and Characterization of Highly Oriented Pure and Gd2O3 Doped CeO2(111) on ZrO2(111)/Al2O3(0001) System”...........................41

Chang HS, GV Korshin, and Z Wang, "Time Resolved Laser Induced Fluorescence Spectroscopy Exploration of Uranyl Adsorption on Gibbsite in $\mathrm{CO}_{2}$

Free Environment”

Du Y, JF Groves, and IV Lyubinetsky, "FIB Directed Self-Assembly of Metal Oxide Nanostructures"

Elliott B, A Boldyrev, and LS Wang, "Computational Investigation of the Structures of the $\mathrm{Fe}_{2} \mathrm{~S}_{2}$ - Anion".

Han JJ, A Li, and HP Lu, "Holding Single Molecules in Solution Using

Magnetic Tweezers: A New Approach to Studying DNA Damage

Recognition by Proteins”.

Lagutschenkov A, G Niedner-Schatteburg, and S Xantheas, ““"The

Spectroscopic Signature of the Transition from "All-Surface" to

"Internally Solvated" Structures in Water Clusters"”,

Laman D and AG Joly, "Measurement of Charge Carrier Lifetimes in $\mathrm{Fe}_{2} \mathrm{O}_{3}$

Thin Films".

Le EA, L Brush, and CH Henegar, "Atomistic Modeling of Helium Clustering and Diffusion along Dislocations in Alpha-Iron"

Ou F, LV Sarat, and DR Baer, "Creating Site-Specitic Nanoporous Silicon

Surface by Using Hydrogen Bubble”. 
Spraggins JM, MV Johnston, and J Laskin, “Large Molecule Characterization via FT-ICR"

Tait SL, Z Dohnálek, CT Campbell, and BD Kay, "n-Alkanes on MgO(100) I:

Coverage-Dependent Desorption Kinetics of n-Butane"

Wang Y, L An, C Wang, and SD Burton, "Thermal Oxidation Behavior of

Polymer-Derived $\mathrm{Si}(\mathrm{Al}) \mathrm{CN}$ Ceramics”,

Wang Y, L An, C Wang, and SD Burton, "Structural Evolution of

Polymer-Derived Si(Al)CN Ceramics".

Yang Z, H Wang, and A Laskin, "Detailed Hygroscopic Study of Sodium

Nitrate Particles by SEM and FTIR"

Yang Z, A Laskin, BG Bunting, JP Cowin, ML Engelhard, PL Gassman, MJ Iedema,

C Wang, and H Wang, "Chemical Characterization of Phosphorous Containing

Soot Particles”

Zeitler TR, AN Cormack, and LR Corrales, "Understanding Chemical Processes of Bioactive Glasses”

Attachment A: Survey 
PNNL-14881

\section{Summary}

PNNL's Management and Operations Contract with the U.S. Department of Energy (DOE) ${ }^{(1)}$ identified an Office of Basic Energy Science (BES) Mission Stretch Goal: “To establish PNNL in a national leadership role in the area of theoretical and experimental condensed phase and interfacial chemical physics research.” Successful achievement of a national leadership role will be evidenced by: "Establishment of a summer school (at least two weeks in duration) to educate graduate students and young scientists in state-of-the-art theory, simulation, and experimental measurement, with a total attendance of at least 60 over the contract period.”

Interfacial and condensed phase chemical physics is not so much a field of study as it is a cross cutting concept. Important issues exist in nearly every scientific discipline, from astronomy to zoology, that fall within the purview of this concept. Likewise, all of the technology and engineering of the modern world and hopes for future development depend to one degree or another on our ability to understand and manipulate condensed phases and interfacial processes.

The guidance from DOE HQ Office of Science gave us considerable latitude in implementing the institute's advanced program that focuses on graduate students, postdoctoral fellows, and university faculty. The institute caters to more senior people than are generally accommodated by PNNL's summer internship programs. We believe that the best education people at this level can receive is actively engaging in research that is relevant to their own career development goals. To accomplish this objective, the institute has a different character and places different requirements on its participants than do either the standard internship programs or other institutes and workshops. Perhaps the best overall description of the institute is a "visiting scientist program for early career researchers." In this spirit, each young scientist's program was individually tailored. The descriptions given below are generally true for most of the young scientists, but may or may not apply to any particular individual.

\section{Participants and the Institute Structure}

Prospective visitors applied electronically at the Summer Research Institute's website, www.pnl.gov/si/. Submission of applications, screening, and correspondence were all handled online. This greatly facilitated the review and screening process as well as record keeping. Preliminary screening was handled by the Director of the Institute, who acknowledged receipt of the applications and informed the candidates of any additional information or actions that were necessary to complete the application process. Several candidates were found to be unsuited for participation in the institute, and they were informed as quickly as that determination could be made. Reasons for rejection at this stage included the following:

(1) Appendix H of Contract Number DE-AC06-76RL01830, Modification M375. 
- Candidate's area of interest was outside the scope of the program, e.g., high-energy physics or genetics.

- Prospective PNNL hosts were already committed or unavailable.

- Candidate was finishing a master's degree or PhD. and did not have a new position.

- Candidate failed to finish the application process.

Candidates who passed this first series of hurdles and who identified a willing PNNL host were then required to submit a summary of proposed research or activity that they would conduct at PNNL during the summer. Normally, this proposal was prepared with the assistance of both the PNNL host and the candidate's academic advisor (as appropriate). Once the application deadline had passed, all completed applications were reviewed by a PNNL science panel consisting of Dr. Donald R. Baer and Dr. L. Rene Corrales of PNNL's Fundamental Science Directorate and Dr. Stephan E. Barlow of PNNL's Environmental Molecular Sciences Laboratory (EMSL). All successful candidates received the unanimous approval of the panel. Individual acceptance and rejection letters were prepared and distributed by the Director. Interestingly, all of the candidates who were accepted agreed to come. However, two candidates were unable to attend the institute for personal reasons. Table 1 provides some statistics on applications and acceptances.

Table 1. 2004 Participant Numbers ${ }^{(a)}$

\begin{tabular}{|l|l|}
\hline Applications and inquiries & 36 \\
\hline Acceptances & 18 \\
\hline Participants & 16 \\
\hline \multicolumn{1}{|c|}{ Graduating Seniors } & $1(2)$ \\
\hline \multicolumn{1}{|c|}{ Graduate Students } & 14 \\
\hline \multicolumn{1}{|c|}{ Postdoctoral Fellows } & 0 \\
\hline \multicolumn{1}{|c|}{ Faculty } & $1(2)$ \\
\hline US Citizens/Foreign Nationals & $8 / 8(9 / 9)$ \\
\hline Academic Institutions & $11(12)$ \\
\hline PNNL Hosts & $14(15)$ \\
\hline (a) Numbers in parentheses refer to acceptances. \\
\hline
\end{tabular}

The application and acceptance procedure requires participation from all concerned parties: PNNL hosts, visitors, and visitors' supervisors at their home institution (if applicable). Further, PNNL hosts were expected to provide appropriate mentoring for the visitors as well as ensure all necessary resources were available to accomplish the proposed work. The visitors bear the bulk of the application responsibility to come to PNNL for an extended time away from home. The visitors' supervisors were expected to continue financial support during this visit to PNNL because the Summer Research Institute normally only covered travel to and from PNNL and partial living expenses. 
Each young scientist had a specific program tailored around his or her schedule and research goals. To accommodate individual schedules, the 2004 summer appointments began in early May, and the final appointment concluded in late September. Before their arrival, each participant was paired with a PNNL host and worked out a research program for the visit. All of this activity was coordinated with the visitor's supervisor. Most often, our visitor was playing a role in a collaboration or prospective collaboration between the PNNL host and his or her supervisor. (For the graduating seniors or faculty members, slightly different procedures applied.) Table 2 lists our visitors, PNNL hosts and home institution supervisors.

Table 2. Interfacial and Condensed Phase Chemical Physics Summer Research Institute PNNL visitors, hosts and academic advisors

\begin{tabular}{|l|l|l|}
\hline \multicolumn{2}{|c|}{ Visitors } & $\begin{array}{l}\text { PNNL Host: } \\
\text { Prof. Lai-Sheng Wang } \\
\text { Physics Dept. } \\
\text { Washington State University } \\
\text { Tri-Cities } \\
\text { Graduate Student } \\
\text { Physical Chemistry } \\
\text { Utah State Univ. } \\
\text { Logan, UT } \\
\text { July 1-30, 2004 } \\
\text { Alexandrova@cc.usu.edu }\end{array}$ \\
\hline Thesis Advisor: \\
Prof. Alexander I. Boldyrev \\
Chemistry Dept. \\
Utah State University \\
Boldyrev@cc.usu.edu
\end{tabular}


Graduate Student

Environmental Engineering

Univ. of Washington

Seattle, WA

June 1 - August 10, 2004

Hschang@u.washington.edu

\begin{tabular}{|l|l}
\hline & \\
\hline Graduate Student &
\end{tabular}

Material Science and

Engineering

Univ. of Virginia

Charlottesville, VA

May 18 - July 23, 2004

yd2f@virginia.edu

Graduate Studen

Physical Chemistry

Utah State University

Logan, UT

July 1 - 30, 2004

slh15@cc.usu.edu

\begin{tabular}{l}
\hline \\
\hline Graduate Student \\
Chemistry \\
Washington State University \\
Pullman, WA \\
June 1 - August 10, 2004 \\
jjh70@wsu.edu
\end{tabular}

Jason J. Han
Jan

Graduate Student

Physical Chemistry

Technical Univ. of

Kaiserslautern

Kaiserslautern, Germany

August 3 - October 4, 2004

Alagut@chemie.uni-kl.de

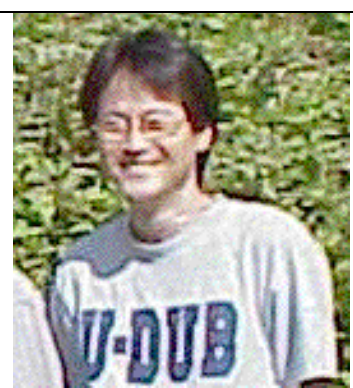
Hyun-shik Chang

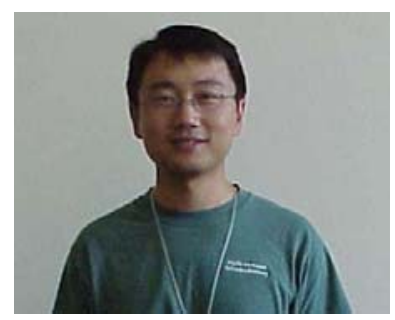

Yingge Du

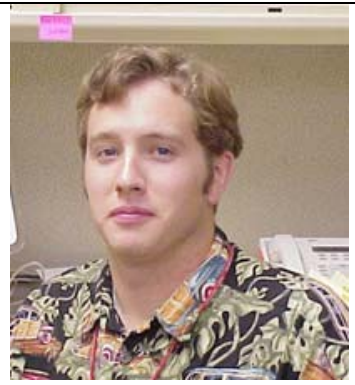

Benjamin M. Elliott
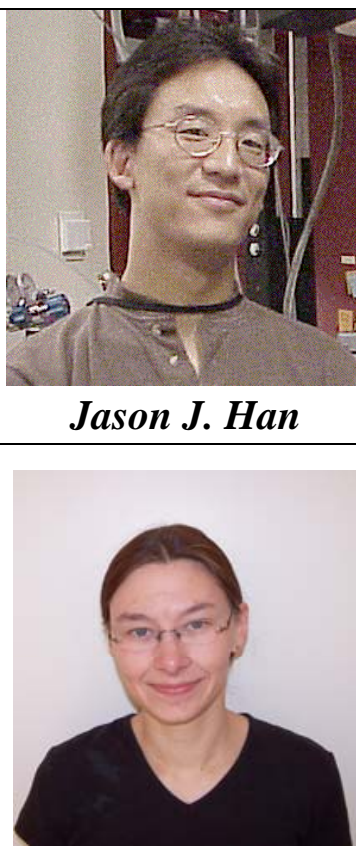

Anita Lagutschenkov
PNNL Host:

Dr. Zheming Wang

FSD, PNNL

zheming.wang@pnl.gov

Thesis Advisor:

Prof. Gregory V. Korshin

Dept of Civil Engineering

University of Washington

korshin@u.washington.edu

\section{PNNL Host:}

Dr. Igor V. Lyubinetsky

FDS, PNNL

Igor.Lyubinetsky@pnl.gov

Thesis Advisor:

Prof. James F. Groves

Materials Science and Engineering

University of Virginia

jfg6e@virginia.edu

PNNL Host:

Prof. Lai-Sheng Wang

Physics Dept.

Washington State University

Ls.wang@pnl.gov

Thesis Advisor:

Prof. Alexander I. Boldyrev

Chemistry Dept.

Utah State University

Boldyrev@cc.usu.edu

PNNL Host:

Dr. H. Peter Lu

FSD, PNNL

Peter.Lu@pnl.gov

Thesis Advisor:

Dr. Alex Li

Chemistry Dept.

Washington State University

dequan@wsu.edu

\section{PNNL Host:}

Dr. Sotiris S. Xantheas

FSD, PNNL

sotiris.xantheas@pnl.gov

Thesis Advisor:

Prof. Gereon Niedner-

Schatteburg

Dept of Chemistry

Technical University of

Kaiserlautern

gns@chemie.uni-kl.de 


\begin{tabular}{|c|c|c|}
\hline $\begin{array}{l}\text { Physics Dept. } \\
\text { Central Washington University } \\
\text { Ellensburg, WA } \\
\text { June 21 - August 29, } 2004 \\
\text { lamand@cwu.edu }\end{array}$ & Iaman & $\begin{array}{l}\text { PNNL Host: } \\
\text { Dr. Alan Joly } \\
\text { FSD, PNNL } \\
\text { agjoly@pnl.gov } \\
\\
\text { Thesis Advisor: } \\
\text { N/A }\end{array}$ \\
\hline $\begin{array}{l}\text { Graduate Student } \\
\text { Materials Science and } \\
\text { Engineering Dept. } \\
\text { University of Washington } \\
\text { Seattle, WA } \\
\text { June } 21 \text { - August 27, } 2004 \\
\text { hhtlle@u.washington.edu }\end{array}$ & & $\begin{array}{l}\text { PNNL Host: } \\
\text { Dr. Howard L. Heinish, Jr. } \\
\text { MSP, PNNL } \\
\text { hl.heinish@pnl.gov } \\
\text { Thesis Advisor: } \\
\text { Prof. Lucien Brush Materials } \\
\text { Science and Engineering } \\
\text { University of Washington } \\
\text { brush@u.washington.edu }\end{array}$ \\
\hline $\begin{array}{l}\text { Graduating Senior } \\
\text { Currently graduate student at } \\
\text { Rensselaer Polytechnic } \\
\text { University } \\
\text { May } 24 \text { - July 30, } 2004 \\
\text { Ouf@rpi.edu }\end{array}$ & & $\begin{array}{l}\text { PNNL Host: } \\
\text { Dr. Laxmikant V. Saraf } \\
\text { FSD, PNNL } \\
\text { Laxmikant.Saraf@pnl.gov } \\
\\
\text { Thesis Advisor: } \\
\text { N/A }\end{array}$ \\
\hline $\begin{array}{l}\text { Graduate Student } \\
\text { Analytical Chemistry } \\
\text { University of Delaware } \\
\text { Newark, DE } \\
\text { July 19 - August 30, } 2004 \\
\text { jms@udel.edu }\end{array}$ & Jeffrey M. Spraggins & $\begin{array}{l}\text { PNNL Host: } \\
\text { Dr. Julia Laskin } \\
\text { FSD, PNNL } \\
\text { Julia.Laskin@pnl.gov } \\
\text { Thesis Advisor: } \\
\text { Prof. Murray V. Johnston } \\
\text { Chemistry and Biochemistry Dept } \\
\text { University of Delaware } \\
\text { mvj@udel.edu }\end{array}$ \\
\hline $\begin{array}{l}\text { Graduate Student } \\
\text { Dept. of Physics } \\
\text { University of Washington } \\
\text { Seattle, WA } \\
\text { June } 16-25,2004 \text { and } \\
\text { August } 16 \text { - 27, } 2004 \\
\text { sltait@u.washington.edu }\end{array}$ & 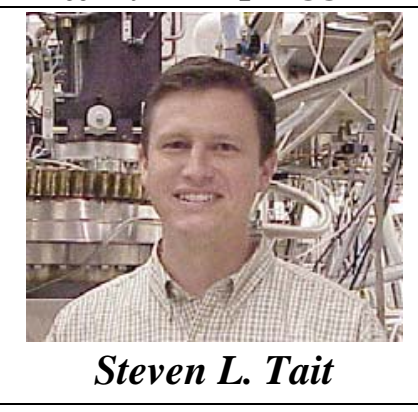 & $\begin{array}{l}\text { PNNL Host: } \\
\text { Dr. Zdenek Dohnalek } \\
\text { FSD, PNNL } \\
\text { Zdenek.Dohnalek@pnl.gov } \\
\text { Supervisor: } \\
\text { Prof. Charles T. Campbell } \\
\text { Dept. of Chemistry } \\
\text { University of Washington } \\
\text { Campbell@chem.washington.edu } \\
\end{array}$ \\
\hline
\end{tabular}




\begin{tabular}{|l|l|l|}
\hline $\begin{array}{l}\text { PNNL Host: } \\
\text { Dr. Chongmin Wang } \\
\text { EMSL, PNNL } \\
\text { Maduate Student } \\
\text { Univ. of Central Florida } \\
\text { Orlando, FL } \\
\text { June 1 - August 10, 2004 } \\
\text { yiguang@ucf.edu }\end{array}$ & $\begin{array}{l}\text { Thesis Advisor: } \\
\text { Prof. Linan An } \\
\text { Advanced Materials Processing } \\
\text { and Analysis Center } \\
\text { University of Central Florida } \\
\text { Lan@mail.ucf.edu }\end{array}$ \\
\hline $\begin{array}{l}\text { Graduate Student } \\
\text { Mechanical Engineering } \\
\text { University of Delaware } \\
\text { June 21 - September 17, 2004 } \\
\text { yangz@me.udel.edu }\end{array}$ & $\begin{array}{l}\text { PNNL Host: } \\
\text { Dr. Alexander Laskin } \\
\text { EMSL, PNNL } \\
\text { Alexander.Laskin@pnl.gov } \\
\text { Thesis Advisor: } \\
\text { Prof. Hai Wang } \\
\text { Mechanical Engineering Dept } \\
\text { University of Delaware } \\
\text { hwang@me.udel.edu }\end{array}$ \\
\hline $\begin{array}{l}\text { Yiguang Wang } \\
\text { Graduate Student } \\
\text { Ceramic Science } \\
\text { Alfred University } \\
\text { Alfred, NY } \\
\text { May 17 - July 23, 204 } \\
\text { zeitletr@alfred.edu }\end{array}$ & $\begin{array}{l}\text { PNNL Host: } \\
\text { Dr. L Rene Corrales } \\
\text { FSD, PNNL } \\
\text { rene.corrales@pnl.gov } \\
\text { Thesis Advisor: } \\
\text { Dean Alastair N. Cormack School } \\
\text { of Engineering } \\
\text { Alfred University } \\
\text { cormack@alfred.edu }\end{array}$ \\
\hline
\end{tabular}

\section{Benefits}

As described above, participation in the Summer Research Institute required a significant investment on the part of all parties involved. The only reason that a young scientist, PNNL host, or academic advisor would be involved is if they believed that their investment would be of benefit. We believe that the institute's structure promoted the likelihood of that success. In addition, there is a less tangible benefit for PNNL that cannot be easily measured, and that is the presence of these energetic, intelligent, well-educated, and highly motivated young scientists. PNNL is in a rather remote location, far from major urban centers and large academic institutions, and these young scientists were an absolute delight to have at the laboratory. Their inquisitiveness, creativity, and naivety helped stimulate PNNL staff to think in new, creative ways. 
PNNL-14881

\section{Measures of Success}

This is a research enterprise. As such, its real success will only become apparent in the coming years and decades. Further, the relatively short time that the young scientists were here can only be a small part of the story. We can already point, however, to a few early successes. First, although the initial applicant pool was limited, we did receive eighteen outstanding applications. Sixteen of the eighteen candidates actually came to PNNL to participate in the institute; therefore, our goal of twelve summer appointments for 2004 was exceeded. Second, all of the candidates, PNNL mentors, and academic advisors expressed enthusiasm about the institute at the outset and were generally more enthusiastic by the end of the respective summer appointments. In one case, the result of this summer's research has already been presented at a national meeting. Several other participants are preparing presentations, and at least six manuscripts are being readied for submission to peer-reviewed journals.

We intend to monitor the progress of the 2004 young scientists and provide an update in the 2005 Annual Report. Among the things we will be monitoring are:

- Establishment of new collaborations

- New research directions and programs

- Publications and presentations

- Intellectual Property.

\section{Participant Survey}

The survey that was sent to all of the summer program participants (visitors, hosts, and advisors) is appended as Attachment A.

\section{Survey Results}

- I received seventeen (17) replies. This group consisted of five PNNL hosts, two academic supervisors, and ten visitors.

- In reviewing these responses, all were enthusiastic about the experience provided by the Summer Research Institute.

- Most of the problems that were encountered are captured by the list of issues and approaches to solving them that we address in the next section. The major complaint was with the red tape and delays associated with foreign nationals and in particular with computer access. This problem was made much worse with the introduction of the VPN EMSL enclave, a computer access program. Interestingly, all of the PNNL staff commented on how smoothly everything seemed to run.

- One visitor did not like the local weather. 
- Approximately half of the participants were already engaged in collaboration and found the Summer Research Institute helpful in furthering their projects.

- The most commonly voiced comment was praise for the availability of resources at PNNL, including instrumentation, computers, and people.

- Another common comment was praise for the friendliness and helpfulness of PNNL staff, particularly those associated with the Summer Research Institute.

- Judging by the surveys and research reports, more that half of the participants are preparing at least one paper for publication — some are actually working on two! Note: No one came to the summer program to write papers per se.

- Several participants report that intellectual property may eventually follow from the work this summer, but no invention disclosures were filed.

- It seems likely that at least two or three new collaborations were established and are likely to continue without additional resources from the institute.

- Most visitors reported that they learned a great deal about instrumentation and computation. In several cases, the breadth of our capabilities allowed combinations of techniques to be brought to bear on a problem in ways not available elsewhere.

\section{Select Comments}

"This was the high-point of my graduate career.” Jormarie Alverez, visitor

"Friendly and smart people, great opportunities to learn a lot from PNNL staff and to establish some network.” Anastassia Alexandrova, visitor

"We already sent this paper for the MRS conference for presentation at Boston Fall Meeting, Nov $29^{\text {th }}$, 2004. Session: Solid State Ionics (K). We are planning to write a journal paper on it in the near future.” Debasis Bera, visitor

Improvement needed: "Miscommunication between the buildings. After finishing the training, my badge wasn't working properly for a long time.” Hyun-shik (Chris) Chang, visitor

"The working hours was 24/7 for me first, but later it was changed to extended hours. Some of us would want 24/7 to make research going well.” Yinnge Du, visitor

“The instrumentation and equipment available were superb.” Ben Elliott, visitor

"The close connection between organizers, mentors and participants. This really helped to solve upcoming problems immediately." Anita Lagutshenkov, visitor 
“Our work has already been accepted by MRS for its fall Meeting at Boston, it will therefore be published as a proceeding paper. It will also be presented on Dec 2nd, 2004 at the meeting in Boston.” Fung Ou, visitor

"I thought the program was very well run-thanks especially to Chris Montgomery. I enjoy working at PNNL because of the opportunities for active discussion with very skilled professional scientists. It was a good opportunity to learn from several scientists. I think an important aspect of this was that I was treated as and expected to perform as a professional scientist. It was good to feel that way and learn about how I need to end up to be an independent professional. I thought it was good that the program was open as far as what project my mentor and I pursued. The program itself got us together to work, then was generally low-key. We were able to work without interruption from the program (i.e., we weren't asked to do a lot of reports or interviews during the summer).” Steven Tait, visitor

"This Summer Institute gave me an invaluable chance to learn many advanced techniques such as SEM, TEM, XPS, FTIR and FT-ICR-MS.” Zhiwei Yang, visitor

“...quantum mechanical calculations involving DFT are now available to me, where they are not available at my school.” Todd Zeitler, visitor

"It is a very good starting point and we are looking forward to more future interactions between WSU and PNNL.” Prof. Alexander Li (thesis supervisor for Jason Han)

"The measurements made during the summer internship helped our research effort out a lot and we anticipate follow up measurements either next winter or summer.” Prof. Murray Johnston (thesis supervisor for Jeff Spraggins)

"This program is a valuable supplement for the research of PNNL staff members and, from what I have been told from the visiting students, a great opportunity for them to be exposed to very diverse aspects of research equipment and groups." Sotiris Xantheas (PNNL host to Anita Lagutshenkov)

"It seemed very well organized. All of the badging, money issues, etc., were handled seamlessly.” Alan Joly (PNNL host to Prof. David Laman)

"Having students on campus for an extended period of time is much better than short visits." Zheming Wang (PNNL host to Chris Chang) 
PNNL-14881

\section{Lessons Learned}

In spite of the general success of the institute, there were a few glitches that we will endeavor to remedy. The most important task is to recognize and preserve the things we did right and build upon them. Further, we must acknowledge that in choosing a particular approach, we necessarily limit other options.

1. Perhaps the most important thing we did right was to recognize that the candidates are not summer students, but rather professional colleagues and collaborators. The entire program is built on this concept.

2. The fact that all of the young scientists were required to make a significant commitment to the program helped ensure that the Summer Research Institute's resources were well utilized.

3. The institute's insistence that each individual summer appointment was well planned in advance also helped to ensure that the duration of the appointment was valuable.

4. The very flexible scheduling of summer appointments maximized the number of candidates who were able to participate.

5. We minimized non-research activities_-perhaps too much, as noted below.

We have identified a number of weaknesses and problems that will be addressed prior to the commencement of the 2005 Summer Research Institute.

1. In the institute's initial publicity, it was unclear that the Summer Research Institute is not a Limited Term Employment Program. With the exception of the graduating seniors who are heading to graduate school, very few candidates will receive full support.

2. The institute is not suited to people finishing a master's degree, Ph.D., or postdoctoral position. The 2005 brochure will be revised to clarify this point.

3. We need to ensure the publicity is available earlier, and we need to get through the application and selection process sooner-preferably by the end of February of each new appointment year.

4. We need to better assess our candidates' probable tax status. Several young scientists were assessed with 30\% tax withholding that made covering local expenses difficult. Additional questions will be added to the application to help in determining this issue.

5. Computer access problems arose as the laboratory was transitioning to a separation of the PNNL and EMSL Network Enclaves. Necessary steps will be taken to ensure computer access problems are resolved prior to the 2005 institute.

6. We should arrange for access to Hanford Technical Library’s electronic resources. 
7. For most of the young scientists, four weeks is too short a duration; this is particularly true for more junior candidates. In most instances, appointments of less than eight weeks should be discouraged.

8. Additional effort must be made to acquaint participants with local social and recreational opportunities. This includes additional institute-sponsored, after-hours activities. Two well-received and successful barbeques were hosted by and held at the home of Steve and Janice Barlow.

9. Establish a lectureship/seminar program.

10. All young scientists were required to write a report of their research in the form of an extended abstract or more formal document. Apparently, many of the young scientists were unfamiliar with this format. More explicit instructions to both the young scientists and PNNL mentors need to be given.

11. Some young scientists found the local transportation system to be inadequate while others were quite pleased. This seems to be a function of expectations and experience. This is a difficult issue that impacts all PNNL visitors. We are working with PNNL's Office of Fellowship Programs to find ways to improve transportation for the young scientists. 
PNNL-14881

\section{Summer Research Institute}

With the knowledge base established in 2004, we will begin planning and preparing for 2005 in October 2004. Publicity for next year's institute will be ready to be distributed by midDecember. Major activities during this period will be updating the brochure to include the changes mentioned above. The application webpage will be updated to incorporate helpful information such as income tax issues. The institute's website and brochure will be reviewed for other improvement opportunities.

In the fall or early-winter, we will make arrangements for two-week seminar programs for well-recognized researchers from outside of PNNL. These researchers would offer four or fivehour tutorial presentations to institute participants, focusing on "Issues and Methods" in the select fields of expertise. These presentations will also be open to the public.

Assuming that our budget remains unchanged, the vetting and selection process in 2005 will be more difficult than 2004 because the pool of highly qualified applicants should expand. This will probably require a prioritization of applicants in addition to whatever scientific merit their proposed activity has. Individuals who participated this year will generally have a lower priority than new applicants - this extends as well to PNNL staff and academic advisors. Further, applicants participating in an already established collaboration will receive lower priority. In 2004, two PNNL mentors each hosted two young scientists. It is unlikely that we will have the resources to repeat this commitment. Matters of prioritization will be formalized with the selection committee before any evaluations are made.

Throughout 2005 and hopefully beyond, we will continue to monitor this year's young scientists and their progress. In particular, we will accumulate data for the "measures of success” listed above.

One of the major changes to the 2005 Summer Research Institute is that Christine Montgomery will no longer be the Administrator. Because Chris accepted another position within PNNL, Nikki Avery will assume her duties. We will miss working with Chris; however, we can be assured that Nikki will serve admirably as the new Administrator. 
PNNL-14881

\section{Technical Reports}

The remainder of this document is devoted to the technical reports provided by each young scientist. As noted above, some of these are single paragraph abstracts and therefore have rather limited content. Other reports, however, are full papers and dissertation chapters. These documents are "as received," apart from minor formatting changes in some cases. We have made no effort to edit or evaluate these reports. Given the limited distribution intended for this report, none of the results in these reports should be considered "published" by virtue of appearing here. 


\title{
Using GEGA algorithm on the MPP2 Supercomputer
}

\author{
Anastassia N. Alexandrova and Alexander Boldyrev \\ Department of Chemistry \\ Utah State University \\ Logan, UT \\ And \\ Lai-Sheng Wang \\ Department of Physics \\ Washington State University, Tri-cities \\ \& Pacific Northwest National Laboratory \\ Richland, WA
}

Worked at the Summer Research Institute July $1^{\text {st }}$ till July $31^{\text {st }}, 2004$.

The primary purpose of the work was to study multiply-aromatic boron clusters potential for the storage of hydrogen. The plan of the work was the following:

1. To find the global minima for boron clusters and corresponding boranes. Development of the automatic method, performing such a search.

2. To clarify the chemical bonding within the species and identify them as aromatic or antiaromatic. Aromatic species should possess special stability, while antiaromatic should be less stable.

3. Using the properties of aromaticity and antiaromaticity of boron clusters and corresponding boranes, to find out when boron clusters can be used as storage devices for hydrogen gas. The proposed driving force would be the loss of aromatic character of the species at the absorption of hydrogen, and gaining of aromaticity, while releasing hydrogen gas.

During the month of July, 2004 the ab initio Gradient Embedded Genetic Algorithm (GEGA) program for finding global minima of clusters, preliminary developed at Utah State University, has been adapted to the NWChem quantum chemistry computational package, available at PNNL. Further adjustments of the code were done in order to use the GEGA on the supercomputer MPP2. Initial testing of the program has been held, and the global minima of $\mathrm{B}_{3}, \mathrm{~B}_{3}^{-}, \mathrm{B}_{4}, \mathrm{~B}_{4}^{-}, \mathrm{B}_{5}, \mathrm{~B}_{5}{ }^{-}, \mathrm{B}_{6}$, $\mathrm{B}_{6}^{-}, \mathrm{B}_{6}{ }^{2-}, \mathrm{B}_{7}, \mathrm{~B}_{7}^{-}, \mathrm{B}_{8}, \mathrm{~B}_{8}^{-}, \mathrm{B}_{9}, \mathrm{~B}_{9}^{-}$boron clusters have been confirmed by means of using GEGA on the MPP2. The global minima were previously identified by means of other methods, and aromaticity and antiaromaticity in them have been detected by molecular orbital analysis. The global minima of boron hydrides, corresponding to the found most stable forms of boron clusters are to be found. Further computational analysis of the possibility to use these compounds for the storage of hydrogen is to be performed. The work is in progress. The major achievement of my work at PNNL is the extensive modification of the GEGA program for the environment available on the supercomputer at PNNL and the NWChem computational package. 


\title{
SOFT-LANDING OF PEPTIDE IONS ONTO SELF-ASSEMBLED MONOLAYER SURFACES
}

\author{
Jormarie Alvarez, ${ }^{a}$ Julia Laskin ${ }^{b}$ and R. Graham Cooks ${ }^{a}$ \\ ${ }^{a}$ Purdue University, Department of Chemistry, West Lafayette, IN \\ ${ }^{b}$ Environmental Molecular Science Laboratory, Pacific Northwest National \\ Laboratories, Richland, WA
}

\section{Introduction}

Soft-landing (SL) is the intact capture of mass-selected polyatomic ions at solid or liquid surfaces. It was introduced in $1977^{[1]}$ and shown unambiguously in the case of small organic ions colliding at self-assembled monolayer (SAM) surfaces. ${ }^{[2]}$ Subsequent demonstration experiments used various types of mass spectrometers including sector analyzers, ${ }^{[3,4]}$ and quadrupoles. ${ }^{[5]}$ The types of ions successfully landed intact were closed-shell small organic ions including those derived from organic dyes. In the biological arena, oligonucleotides have been successfully soft landed by mass spectrometry and detected by PCR. ${ }^{[6]}$ Even ionized viruses have been soft landed with retention of viability. The first such experiments were performed without mass analysis ${ }^{[7]}$ but time-of-flight (TOF) mass analysis ${ }^{[8]}$ has since been used. Most recently, soft landing of proteins has been demonstrated and introduced as a method with potential application in generating protein microarrays. ${ }^{[5]}$ In these experiments, mass spectrometry serves as the method of separation, providing a separation procedure based on physical principles which are very different from those encountered in more conventional separation methods. Experimental conditions have been found under which the proteins retained their biological activity when soft-landed.

The studies of soft landing of small ions have yielded considerable information on the process. There is an optimum collision energy for a given ion and surface: if the energy is too low the efficiency of landing is low presumably because scattering from the surface dominates; if it is too high, surface-induced dissociation (SID) or molecular rearrangement occurs. In the case of dissociation the products may be retained at the surface ("crash landing") or scattered into the gas-phase. The nature of the surface is very important because of its effect on the efficiency of energy transfer, ${ }^{[9-12]}$ well-known from studies of inelastic collisions. The choice of surface also affects whether the landed ion is or is not neutralized. ${ }^{[13,14]}$ Evidence has been provided ${ }^{[2,5,15,16]}$ that a fluorinated selfassembled monolayer (F-SAM) surface can allow soft landing with retention of the charge state of the gas phase ion. There is also evidence ${ }^{[17]}$ that this more demanding phenomenon - intact soft landing of the molecular ion - can occur with some proteins, again using inert (hydrophobic) F-SAM surfaces. The efficiency of soft landing, defined as the ratio of ions released to number of ions dosed, is not well characterized since it is hard to separate this from the efficiency of ionization which is always extremely low and the efficiency of the method used to analyze the soft-landed ions. Nevertheless, landing 
efficiencies of $0.5 \%$ and $5 \%$ have been estimated for small organic ions ${ }^{[15]}$ and proteins, ${ }^{[5]}$ respectively.

Here we report extension of the soft-landing experiment to the case of peptides. Since both small organic ions and proteins are known to soft land successfully, it was expected that peptides would do so too. In fact, a phenomenon observed during systematic studies of peptide ion collisions with surfaces suggested that soft-landing was occurring. This observation was the lack of any scattered ions over a wide range of collision energies near threshold. These experiments are done using an ion cyclotron resonance instrument (FT-ICR) which has been used extensively to study the collisions of mass-selected ions with specific surfaces, especially SAM surfaces. For the present study the FTICR instrument was further modified to allow in situ SIMS analysis of the surface after soft landing. The SIMS source was a Cs ion gun with $2 \mathrm{keV}$ primary ion energy. Independent high-resolution $25 \mathrm{kV} \mathrm{Ga}{ }^{+}$ToF-SIMS analysis of soft-landed samples was also performed for comparison with SIMS analysis performed using the modified FTICR.

\section{Experiments and Methodology}

\section{Soft-Landing Experiments}

\section{Fourier Transform Ion Cyclotron Resonance Mass Spectrometer (FTICR-MS)}

Soft-landing experiments were performed in a custom built 6-T FT-ICR mass spectrometer (Pacific Northwest National Laboratory, Richland, WA) described elsewhere (Figure 2.1). ${ }^{[18]}$ A syringe pump (Cole Parmer, Vernon Hills, IL) was used for direct infusion of the electrospray sample at a flow rate of $0.3 \mu \mathrm{L} / \mathrm{min}$. Protonated peptides are formed in an external electrospray ionization (ESI) source illustrated in Figure 2.2. Efficient transmission of ions into the vacuum system is achieved using an electrodynamic ion funnel. After the ion funnel, ions undergo collisional relaxation in a collisional quadrupole (CQ) followed by mass selection using a commercial Extrel quadrupole mass filter (resolving quadrupole, RQ). The mass-selected ions are transmitted through a third quadrupole (accumulation quadrupole, AQ) operated in the rfonly mode into an electrostatic ion guide that consists of a series of five tube lenses which allow for precise positioning and shaping of the ion beam. An electrostatic quadrupole bender is located after the second tube lens to avoid contamination of the surface by preventing neutral molecules from reaching the surface. After exiting the last tube lens selected ions are transported into the ICR cell through a long flight tube. Ions are decelerated into the strong magnetic field by two deceleration plates located at the entrance of the ICR cell. Soft-landing occurs when selected ions are made to collide at various energies with a surface positioned by a vacuum interlock assembly at the rear trapping plate of the ICR cell. During soft-landing experiments static dc potentials are applied to the front trapping plate, the ring electrode and the rear trapping plate of the ICR cell. A potential diagram of the ion soft-landing mode of the FTICR is shown in Figure 2.3. The collision energy of multiply-charged ions was adjusted by changing the dc offset applied to the rear trapping plate of the ICR cell. The soft-landing spot size was determine to be about $4 \mathrm{~mm}^{2}$ by ToF-SIMS imaging of the surface after soft-landing. The surface current was monitored using a picoammeter. 


\section{Surface Analysis after Soft-Landing}

Secondary Ion Mass Spectrometry - Fourier Transform Ion Cyclotron Resonance Mass Spectrometer (SIMS-FTICR)

After ion deposition, the surface was subjected to in situ $\mathrm{Cs}^{+}$ion desorption analysis within 5 minutes of ion soft-landing. The last of the five tube lenses (Lens 5) of the FTICR electrostatic ion guide is mounted in a custom built moving stage and can be interchanged with a Cesium ion gun for SIMS analysis of the surface after soft-landing. $\mathrm{Cs}^{+}$ions are generated with a cesium ion gun, transported into the ICR cell through a long flight tube and collided with the newly modified surface, positioned at the rear trapping plate of the ICR cell. Static SIMS conditions with a ion flux of about $1.5 \times 10^{10}$ ions $/ \mathrm{cm}^{2}$ per cycle ( 25 shots) were achieved when the following potentials were applied to various focusing elements: $\mathrm{Cs}^{+}$gun floating voltage, $+2000 \mathrm{~V} ; \mathrm{Cs}^{+}$ion gun shield, $+1909 \mathrm{~V}$; short flight tube, $+850 \mathrm{~V}$; long flight tube, $-650 \mathrm{~V}$; and deceleration plates, $0 \mathrm{~V}$. A potential diagram of the SIMS analysis mode on the FTICR is shown in Figure 2.5. The potential applied to the $\mathrm{Cs}^{+}$gun was provided by a power supply that was floated to the desired voltage. Data acquisition was accomplished with a MIDAS data station. The $\mathrm{Cs}^{+}$ion beam was pulsed by alternating the potential applied to the flight tube between $650 \mathrm{~V}$ to $+2300 \mathrm{~V}$, the latter serving to block the $\mathrm{Cs}^{+}$beam from reaching the surface. The pulsed width was $100 \mu$ s and the surface was exposed for 25 shots $(1$ cycle). Scattered ions are captured by raising the potentials on the front and rear trapping plates of the ICR cell by 30 volts. In order to increase the sensitivity excitation events were used - to expel $\mathrm{Cs}^{+}(133 \mathrm{Th})$ and $\mathrm{Au}^{+}(197 \mathrm{Th})$ ions from the ICR cell - prior to the final excitation/detection event. The time elapse between the trapping event and the excitation/detection event was about $0.4 \mathrm{~s}$.

Time of Flight- Secondary Ion Mass Spectrometry (ToF-SIMS)

Ex situ of analysis of the surfaces modified using the FTICR was performed using a PHI TRIFT ToF-SIMS mass spectrometer (Physical Electronics, Eden Prairie, MN), Figure 2.6. High-resolution $25 \mathrm{kV} \mathrm{Ga}^{+}$ToF-SIMS analysis was performed using a beam current of $60 \mathrm{pA}$ for 120 seconds at a frequency of $10 \mathrm{kHz}$ and a primary pulse width of $1 \mathrm{~ns}$. The experimental conditions were set for static SIMS analysis at an ion flux of about $4.5 \times$ $10^{9}$ ions $/ \mathrm{cm}^{2}$ and a spot size of $100 \times 100 \mu \mathrm{m}^{2}$. In order to confirm static SIMS conditions a modified surface was bombarded for a period of 30 minutes. The signal of the peptide was monitored during this time and only a $30 \%$ decrease in the signal was observed. 


\section{Results and Discussion}

\section{Separation of Peptides by Ion Soft-Landing}

The ability to selectively deposit peptides onto organic monolayers using a mass spectrometer was investigated by peptide ion soft-landing using a Fourier transform ion cyclotron resonance mass spectrometer. The desired peptide ion was mass-selected from a mixture of peptide ions produced by electrospray ionization and deposited into an FSAM surface. Successful deposition of the peptide onto the surface was determined by static SIMS analysis of the surface after ion soft-landing. Figure 2.7 shows the FTCIR mass spectrum of a tertiary mixture of peptides ionized by electrospray ionization. The spectrum shows doubly-protonated peptide ions of bradykinin $(\mathrm{m} / \mathrm{z} 530)$, gramicidin $\mathrm{s}$ $(\mathrm{m} / \mathrm{z} 571)$ and substance $\mathrm{p}\left(\mathrm{m} / \mathrm{z}\right.$ 674). Figure 2.8 shows the $2 \mathrm{keV} \mathrm{Cs}{ }^{+}$SIMS mass spectrum obtained after isolation of doubly-charged gramicidin $\mathrm{s}(\mathrm{m} / \mathrm{z}$ 571.6) from the mixture using the resolving quadrupole and then onto an FSAM surface. Mass-selection of the peptide was performed with a 4 Th isolation window. The SIMS mass spectrum shows singly-protonated gramicidin $\mathrm{s}(\mathrm{m} / \mathrm{z} 1141)$, the gold cationized gramicidin $\mathrm{s}$ $[\mathrm{M}+\mathrm{Au}]^{+}$as well as some peptide fragment ions labeled with $(*)$. Notice that molecular ions of bradykinin $(\mathrm{m} / \mathrm{z} 1060)$ or substance $\mathrm{p}(\mathrm{m} / \mathrm{z} 1347)$ are not observed in the mass spectrum in Figure 2.8. In a second experiment, the doubly-charged molecular ion of substance $\mathrm{p}(\mathrm{m} / \mathrm{z}$ 674.3) was independently mass-selected from the peptide mixture and deposited onto an FSAM surface. Figure 2.9 shows the $2 \mathrm{keV} \mathrm{Cs}^{+}$SIMS mass spectrum obtained after selective deposition of doubly-charged substance $p$ onto the surface. Again, the SIMS mass spectrum shows mostly the singly-protonated peptide $(\mathrm{m} / \mathrm{z}$ 1347.7) and the gold molecular cation of the peptide $[\mathrm{M}+\mathrm{Au}]^{+}$. Peptide fragments of substance $\mathrm{p}$ with small relative intensities were also observed in addition to a modestly abundant intact ion, $[\mathrm{M}+2 \mathrm{H}]^{2+}$. However, no molecular ions of bradykinin or gramicidin $\mathrm{s}$ are observed in the SIMS spectrum of deposited substance $\mathrm{p}$. The results show that one can selectively deposit a peptide onto a surface using a mass spectrometer without contamination by other ions provided that the ions in the mixture have different mass-tocharge ratios and are completely resolved in the mixture mass spectrum.

In addition to peptide fragment ions, the SIMS mass spectrum of the FSAM surface also shows gold cluster ions $\left(\mathrm{Au}_{2}{ }^{+}\right.$and $\left.\mathrm{Au}_{3}{ }^{+}\right)$and gold/sulfur cluster ions $\left(\mathrm{Au}_{2} \mathrm{SH}^{+}\right.$ and $\mathrm{Au}_{3} \mathrm{~S}^{+}$). The presence of this ion is evidence of the strong Au-S bond at the metalthiol interface. A large variety of gold containing ions such as $\mathrm{AuCF}_{2}{ }^{+}$and $\mathrm{Au}_{2} \mathrm{~F}^{+}$are also observed but with small relative intensities upon $\mathrm{Cs}^{+}$desorption ionization of the self assembled monolayer. This agrees with previous static SIMS studies of fluorinated SAMs surfaces performed in a quadrupole-based instrument with $\mathrm{Xe}^{+}$at a primary beam energy of $7.0 \mathrm{keV}$. The static negative ions SIMS mass spectrum shows prominent fluorinecontaining fragments such as $\mathrm{AuF}_{2}^{-}, \mathrm{Au}_{2} \mathrm{~F}^{-}$and $\mathrm{HAu}_{2} \mathrm{CF}_{2}^{-}$, however no molecular ions are present in the spectrum in contrast to the case for alkanethiol $\left(\mathrm{M}=\mathrm{C}_{\mathrm{n}} \mathrm{H}_{\mathrm{n}+1} \mathrm{SH}\right)$ surfaces on gold ${ }^{[20]}$ SIMS spectra of SAM surfaces of alkanethiol $\left(\mathrm{M}=\mathrm{C}_{\mathrm{n}} \mathrm{H}_{\mathrm{n}+1} \mathrm{SH}\right)$ on gold show a rich variety of Au-molecular cluster ions such as $\mathrm{Au}[\mathrm{M}-\mathrm{H}]_{2}^{-}, \mathrm{AuM}^{-}, \mathrm{AuSM}^{-}$and $\mathrm{Au}_{2}[\mathrm{M}-\mathrm{H}]^{-}$in addition to gold-sulfur cluster ions such as $\mathrm{AuS}^{-}, \mathrm{AuS}_{2} \mathrm{H}^{-}$, and $\mathrm{Au}_{2} \mathrm{~S}^{-}{ }^{[20]}$ 


\section{In situ SIMS-FTICR Analysis of FSAM Surfaces after Peptide Deposition}

The peptides investigated by ion-soft-landing and subsequently analyzed by SIMS-FTICR were singly-charged leucine enkephalin $(\mathrm{m} / \mathrm{z} 556)$, angiotensin III $(\mathrm{m} / \mathrm{z}$ 932), RVYIFPF (m/z 942), des-arg ${ }^{1}$-bradykinin (m/z 904), and des-arg ${ }^{9}$-bradykinin $(\mathrm{m} / \mathrm{z}$ 904); doubly-charged bradykinin (m/z 530), gramicidin $\mathrm{s}(\mathrm{m} / \mathrm{z} 571)$ and substance $\mathrm{p}(\mathrm{m} / \mathrm{z}$ 674); and triply-charged renin substrate porcine $(\mathrm{m} / \mathrm{z} 587)$ and melittin $(\mathrm{m} / \mathrm{z}$ 950). The nomenclature used to identify the peptide fragment ions is based in that proposed by Roespstorff and Fohlmann ${ }^{[21]}$ and later modified by Biemann ${ }^{[22]}: N$-terminal ions $\left(a_{n}, b_{n}\right.$, $c_{n}$, and $\left.d_{n}\right) ; C$-terminal sequence ions $\left(x_{n}, y_{n}, z_{n}, v_{n}\right.$, and $\left.w_{n}\right)$; immonium ions (e.g. $R{ }^{+} \mathrm{NH}_{2}$ $=\mathrm{CH}[$ arginine side chain $]$ ) and internal ions (e.g. $P F$ [H-proline-NH-CH(phenylalanine side chain) $\left.-\mathrm{C} \equiv \mathrm{O}^{+}\right]$).

Bradykinin: Figure 2.10 shows a mass spectrum after deposition of doublycharged bradykinin $[\mathrm{M}+2 \mathrm{H}]^{2+}(\mathrm{m} / \mathrm{z}$ 530.7) on an FSAM surface as analyzed in situ by 2 $\mathrm{kV} \mathrm{Cs}^{+}$SIMS-FTICR. The peaks characteristic of the surface are omitted for simplicity. Soft-landing was performed at $30 \mathrm{eV}$ collision energy for 16 minutes at an average current of $30 \mathrm{pA}$ measured at the surface. An average of the ten lowest energy structures of doubly-charged bradykinin as investigated by molecular calculations results in a crossection of about $241 \AA^{2} .^{[23,24]}$ Under these conditions and with a soft-landing spot size of $4 \mathrm{~mm}^{2}$ the surface was exposed to a total of $2.3 \times 10^{10}$ ions $/ \mathrm{mm}^{2}$ or the equivalent to about $1.3 \%$ of a monolayer. A peak corresponding to the protonated peptide $[\mathrm{M}+\mathrm{H}]^{+}$ $(\mathrm{m} / \mathrm{z} 1060.5)$ and a series of peaks corresponding to fragment ions of the peptide are observed.

The SIMS-FTICR spectrum of soft-landed bradykinin reveals unambiguously the nature of all fragment ions and confirms the amino acid sequence of the peptide as ions are observed from both the $\mathrm{C}$ - and the $\mathrm{N}$-termini. By far, the major product ion in the SIMS-FTICR spectrum shown in Figure 2.10 is $\mathrm{b}_{2}$. This fragment ion is also distinctively observed during BIRD dissociation of singly-protonated bradykinin at $200^{\circ} \mathrm{C}$ with $30 \mathrm{~s}$ reaction delay. BIRD dissociation of singly-charged bradykinin results primarily in the loss of $\mathrm{NH}_{3}$ and some production of $\mathrm{b}_{2} / \mathrm{y}_{7}{ }^{\left[{ }^{[25]}\right.}$ In BIRD studies with $30 \mathrm{~s}$ reaction delay the singly protonated peptide $[\mathrm{M}+\mathrm{H}]^{+}$which produces $\mathrm{b}_{2}$ has dissociated significantly by the loss of ammonia $\left[\mathrm{M}-\mathrm{NH}_{3}+\mathrm{H}\right]^{+}$and thus $\mathrm{b}_{2}$ cannot be produced in high abundance. However, during the SIMS-FTICR experiments described here the singly-protonated ion $[\mathrm{M}+\mathrm{H}]^{+}$does not have enough time to loose ammonia in significant amounts and thus the overwhelming presence of $b_{2}$ in the spectrum. However, $y_{7}-$ the ion complementary to $b_{2}$ - is not as abundant as $b_{2}$ in the SIMS-FTICR spectrum. This suggests that further fragmentation of $\mathrm{y}_{7}$ may occur and the presence of internal fragment ions such as PG and PGF in the SIMS-FTICR mass spectrum points in that direction.

Substance p: Another peptide investigated through soft-landing onto an FSAM surface was substance p. Figure 2.11 shows a mass spectrum of an FSAM surface after deposition of doubly-charged substance $\mathrm{p}[\mathrm{M}+2 \mathrm{H}]^{2+}$ as analyzed in situ by $2 \mathrm{keV} \mathrm{Cs}{ }^{+}$ SIMS-FTICR. Notice that the difference between Figure 2.9 and Figure 2.11 is that in the latter the peaks characterstic of the surface are omitted for simplicity. For the case of substance $\mathrm{p}$, soft-landing was performed at $30 \mathrm{eV}$ collision energy for 48 minutes at an average current of $10 \mathrm{pA}$ measured at the surface. An average of the ten lowest energy structures of doubly-charged substance $\mathrm{p}$ as investigated by molecular calculations results 
in a crossection of about $299 \AA^{2}$. Under these conditions with a soft-landing spot of 4 $\mathrm{mm}^{2}$ the surface was exposed to a total of $2.3 \times 10^{10}$ ions $/ \mathrm{mm}^{2}$ or the equivalent of about $1.7 \%$ of a monolayer. Again, the singly-protonated peptide ion $[\mathrm{M}+\mathrm{H}]^{+}$is observed in the SIMS-FTICR mass spectrum of the modified FSAM surface in addition to peptide fragment ions. The SIMS-FTICR spectrum contain primarily internal fragment ions and some minor production of $a$ and $b$ ions. The latter is expected from the position of an arginine in the N-terminus of the peptide. However, internal fragmentation indicates that a significant amount of internal energy is deposited into the peptide ion upon $2 \mathrm{keV} \mathrm{Cs}^{+}$ desorption resulting in multiple bond cleavages most likely through a consecutive decomposition pathway.

Notice that SIMS analysis of the FSAM surfaces modified by landing the doublyprotonated peptide $[\mathrm{M}+\mathrm{H}]^{+}$reveals the presence of mostly the singly-protonated peptide. Only a small peak corresponding to the doubly-protonated ion is observed in the SIMS mass spectrum of soft-landed substance $p$ Figure 2.11. This indicates that the peptide is being oxidized either by the surface or during the sputtering process; both possibilities will be discussed later on this chapter.

\section{Effect of the Landing Energy of the Peptide}

Questions arise as to whether the fragmentation observed for these peptides are primarily a result of "crash landing" - projectile ion dissociation upon impact with some of the fragments retained by the organic monolayer - as observed for small organic ions ${ }^{[15]}$ or the result of internal energy deposition onto the intact peptide ion by $\mathrm{keV}$ ion desorption during the SIMS analysis step. To test if the fragmentation observed is the result of "crash-landing" the effect of the kinetic energy of the peptide upon deposition onto the surface was investigated. Soft-landing of peptides at collision energies ranging from 0 to $150 \mathrm{eV}$ was performed and the modified surface analyzed by static SIMS. Figure 2.12 shows SIMS mass spectra of bradykinin deposited onto a fluorinated SAM surface at $20 \mathrm{eV}$ (Figure 2.12a) and at $150 \mathrm{eV}$ (Figure 2.12b). If dissociation is occurring upon impact at the surface we would expect to observe an increase in fragment ion abundance with soft-landing energy. However, soft-landing of doubly-charged bradykinin at $150 \mathrm{eV}$ collision energy results in similar amount of fragmentation than that observed at $20 \mathrm{eV}$. In fact, for two peptides investigated, bradykinin and substance $\mathrm{p}$, the amount of fragmentation observed was independent of the energy of the soft-landed peptide ion beam. Hence no evidence of "crash landing" was observed upon static SIMS analysis of modified surfaces when the collision energy of the deposited peptide was varied. The lack of change in peptide fragmentation abundance with increasing energy of the primary peptide ion beam upon deposition confirmed that the peptide fragments observed are the result of the method used to sputter the peptide from the surface - $\mathrm{Cs}^{+}$ static SIMS - and not due to "crash landing" of the peptide upon deposition onto the surface. However, the "crash landing" phenomenon has been observed previously during soft landing of $\left(\mathrm{CH}_{3}\right)_{3} \mathrm{SiOSi}\left(\mathrm{CH}_{3}\right)_{2}{ }^{+}\left(\mathrm{m} / \mathrm{z}\right.$ 147) on FSAM surfaces. $\mathrm{Xe}^{+}$sputtering spectrum not only revealed the intact parent ion but also fragment ions $\left(\mathrm{CH}_{3}\right)_{3} \mathrm{Si}^{+}(\mathrm{m} / \mathrm{z}$ 73), $\mathrm{CH}_{3} \mathrm{SiH}_{2}{ }^{+}\left(\mathrm{m} / \mathrm{z}\right.$ 45) and $\mathrm{C}_{3} \mathrm{H}_{9} \mathrm{OSi}_{2}{ }^{+}\left(\mathrm{m} / \mathrm{z}\right.$ 117) ${ }^{[15]}$ Studies at different sputtering energies confirmed that fragmentation of the deposited ion in this case occurred upon initial impact and not at the $\mathrm{Xe}^{+}$sputtering stage of the experiment. 
Although no significant differences in fragmentation of deposited peptides at various soft-landing energies was observed upon static SIMS analysis the amount of singly-protonated peptide sputtered from the surface upon $2 \mathrm{kV} \mathrm{Cs}^{+}$SIMS analysis varied significantly with soft-landing energy. Substance $\mathrm{p}[\mathrm{M}+2 \mathrm{H}]^{2+}$ was accumulated at 5 minutes intervals with a measured surface current of $6 \mathrm{pA}\left(6 \times 10^{9}\right.$ ions per 5 minutes interval) onto an FSAM surface. The soft-landing energy was increased in $25 \mathrm{eV}$ intervals from $0 \mathrm{eV}$ to $150 \mathrm{eV}$. The results are shown in Figure 2.13a, the number of ions accumulated on the surface was controlled so as to not approach saturation levels of the surface which would have made it impossible to distinguish soft-landing energy effects from saturation effects.

The first derivative of the plot shown in Figure 2.13a is shown in Figure 2.13b, this plot represents the change in the total amount of peptide deposited as a function of soft-landing energy. If the soft-landing energy plays no role in the amount of peptide that can be deposited onto an FSAM surface we would expect Figure 2.13a to be a linear plot and its derivative to be a constant. However, Figure 2.13b shows that higher amounts of intact peptide are sputtered from the surface when low energies $(<50 \mathrm{eV})$ are used for ion soft-landing. The amount of sputtered intact peptide decreases with increasing energy of ion soft-landing. This behavior was also observed in the case of bradykinin and gramicidin s. Soft-landing studies of small organic molecules also demonstrated that at very low energies $(<10 \mathrm{eV})$ the soft-landing process is favored since other ion/surface collision channels are suppressed and as the energy increases the possibility of softlanding decreases. ${ }^{[15]}$

\section{Cesium Desorption: Kinetic Energy Effects}

The $\mathrm{Cs}^{+}$ion collision energy used for SIMS analysis of the modified surface was also varied and the fragmentation of the peptide was monitored, the results are summarized in Table 2.1. SIMS analysis with $500 \mathrm{eV} \mathrm{Cs}^{+}$and $1 \mathrm{keV} \mathrm{Cs}^{+}$results in total dissociation of the protonated peptide. Analysis with $2 \mathrm{keV} \mathrm{Cs}^{+}$shows a peak that corresponds to the protonated peptide in addition to some peptide fragment ions. This is indicative of a change in sputtering mechanism as the $\mathrm{Cs}^{+}$energy increases; at low energies $(500 \mathrm{eV}$ and $1 \mathrm{keV})$ enough internal energy is deposited into the soft-landed peptide to cause its complete dissociation. On the other hand, at $2 \mathrm{keV}$ the soft-landed peptide is ejected from the surface with much less internal energy so that it can be detected. The kinetic energy effect described here has been previously observed for the case of peptides and small molecules ionized by static SIMS using $\mathrm{Cs}^{+}$primary ions. ${ }^{[26,27]}$ It was observed that the extent of fragmentation increased as the translational energy of the bombarding primary ion is decreased. These results are interpreted as a consequence of changes in the internal energy deposition by the bombarding particle. At higher primary ion energies, less fragmentation is observed due to desorption of a greater number of low-energy molecular ions than of higher-energy ions. ${ }^{[28]}$ This has been explained as a result of the penetration depth of the primary ion increasing approximately linearly with collision energy while the affected volume increases cubically. ${ }^{[26]}$ 


\section{SIMS Sputtering Yield of Soft-Landed Peptides}

Various peptides of different sizes and amino acid composition were soft-landed on FSAM surfaces and analyzed by in situ $2 \mathrm{keV} \mathrm{Cs}^{+}$static SIMS on the FTICR. SIMS mass spectra of each of the modified surfaces show mostly singly-protonated peptide ions in addition to some peptides fragments. If one assumes that all peptides investigated have the same soft-landing efficiency under the given experimental conditions then differences in the amounts of sputtered peptide during SIMS analysis are attributed to the ability of 2 $\mathrm{keV}$ Cs primary ion beam to successfully sputter the peptide ion from the surface. Table 2.2 shows the relative amounts of singly-charged peptide sputtered from modified FSAM surfaces under the same experimental conditions. The amount of sputtered peptide was calculated as the ratio between the ion intensity of the peak corresponding to the singlyprotonated peptide $[\mathrm{M}+\mathrm{H}]^{+}$and the peak intensity of an ion characteristic of the surface, $\mathrm{Au}_{3}{ }^{+}(\mathrm{m} / \mathrm{z}$ 591). It is well-known that secondary ion yields of organic molecules during SIMS analysis mainly depend on molecular weight, chemical structure and the nature of the matrix from which these molecules are desorbed. For the peptides investigated here the matrix is the same - FSAM surface - so any differences in ion sputtering yield can be attributed to the chemical structure or molecular weight of the peptide. Benninghoven studied the influence of primary structure on the sputtering yields of a series of peptides in the mass range between 500 and 1900 Da prepared as monolayers on various metallic surfaces. In his studies Benninghoven correlated the emission of molecular ions $[\mathrm{M}+\mathrm{H}]^{+}$ with the presence of free amino groups at the $\mathrm{N}$-terminus or in the side chain of basic amino acids Arginine (R) and Lysine (K). A comparison of peptides with varying numbers of basic amino acids (Arg and Lys) shows that the yield of molecular ions decreases with increasing the number of basic residues on the peptide. For the peptides investigated here there is no obvious correlation between the sputtering yield and the presence of free amino groups. For example, Substance $p$ an undecapeptide containing three amino groups (a terminal amino group, one arginine and one lysine) has much higher sputtering yields than leucine enkephalin, a pentapepide with only one amino group on the N-termini. Moreover, an almost exponential drop of molecular ion yields with mass has been observed for peptides electrosprayed on surfaces. ${ }^{[29]}$ However, substance $p$ is among the larger peptides studied here. Another surprising case is that of gramicidin $\mathrm{s}$, Bennighoven found no protonated molecular ions for gramicidin $\mathrm{s}$ electrosprayed on various surfaces which he attributed to the blocked N-terminus of the cyclic peptide and the absence of basic residues, instead he observed cationized molecular ions $\left([\mathrm{M}+\mathrm{Na}, \mathrm{K}]^{+}\right.$and $\left.[\mathrm{M}+\mathrm{Ag}]^{+}\right)$of gramicidin s. However in the case of softlanding experiments on FSAM surfaces, a strong peak corresponding to singlyprotonated gramicidin $\mathrm{s}$ is observed upon SIMS analysis. We believe that this is due to the inert nature of the FSAM surface which allows the ion to retain its charge and thus ionization of the peptide is not necessary in order to be observed upon SIMS analysis. Finally, SIMS analysis of a surface modified with Renin Substrate Porcine results in the lowest molecular sputtering yield of the peptides investigated even though it contains only one basic amino acid residue. However, the low sputtering yield obtained for Renin Substrate Porcine can be a direct result of its high molecular weight.

The relative amount of sputtered peptides was also investigated for HSAM, HOOC-SAM and gold surfaces. The results show that SIMS sputtering yields of soft- 
landed peptides are strongly dependent on the kind of surface used for deposition. For example, angiotensin III shows a relatively high sputtering yield from FSAM surfaces, however the sputtering yield of angiotensin III was reduced to about $25 \%$ when the analysis was performed on a HSAM surface. Further reduction to about $4 \%$ was observed on bare gold surfaces and only about $1 \%$ relative yield was obtained upon analysis from a HOOC-terminated SAM surface. As mentioned above, we believe that intrinsic properties of the FSAM surfaces are responsible for allowing the peptide to retain its charge. Moreover, for the case of small organic ions it was observed that intact ions maintain their charge in FSAM surfaces for long periods of time. ${ }^{[2,15,16]}$ This observation was attributed to the highly inert nature of these hydrophobic surfaces. ${ }^{[30-32]}$

The decrease in ion yields observed for HSAM surfaces can be attributed to the less hydrophilic and not so organized assembly of these surfaces when compared to FSAM surfaces. The somewhat weaker assembly might allow impurities to be retained on the organic web that can in principle neutralize the soft-landed ion reducing the ion yields observed upon SIMS sputtering from these surfaces. In the case of bare gold surfaces neutralization is very facile through electron transfer between the metallic substrate and the soft-landed ion.

\section{Amount of Peptide Deposited upon Soft-Landing: Surface Saturation}

If in fact ions retain their charge upon deposition into FSAM surfaces one can expect that saturation due to Coulombic repulsion will occur long before deposition of one monolayer of the peptide. Experiments were performed in which bradykinin $[\mathrm{M}+2 \mathrm{H}]^{2+}$ was accumulated on an FSAM surface at deposition intervals of $10 \%$ of a monolayer, the results are shown in Figure 2.14a. For the case of bradykinin saturation occurred upon deposition of about $60 \%$ of a monolayer. This result supports the hypothesis that the ions retain some of their charge upon soft-landing on FSAM surfaces. Substance $p$ accumulation on an FSAM surface was also investigated; the results are shown in Figure 2.14b. Notice that in the case of substance $\mathrm{p}$ saturation of the surface occurred within deposition of only about $20 \%$ of a monolayer, much earlier than in the case of bradykinin. One can try to explain this observation by differences in Coulombic repulsion exerted by each peptide based on their ability to be neutralized on the FSAM matrix. The late saturation observed for bradykinin could be the result of this peptide being neutralized more readily than substance $\mathrm{p}$ on the FSAM surface; this would result in less Coulombic repulsion and thus later saturation. If bradykinin is neutralized more promptly on the FSAM surface than substance $p$ this phenomenon would also help to explain the relatively low SIMS sputtering ion yields obtained for bradykinin - among the smallest from the series of peptides that were investigated (Table 2.2). However, according to Cassidy and coworkers bradykinin ${ }^{[33]}$ and substance $\mathrm{p}^{[34]}$ have very similar gas-phase acidities, $225.8 \pm 4.2 \mathrm{kcal} \mathrm{mol}^{-1}$ and $226.4 \pm 3.6 \mathrm{kcal} \mathrm{mol}^{-1}$, respectively; and one would expect that if neutralization is taking place it would affect both peptides to the same extent. Another possibility is simply that substance $\mathrm{p}$ can accumulate better than bradykinin on the FSAM surface and thus saturation appears much earlier. Innumerable studies on the dynamics of ion-surface interactions point to the fact that various processes compete during ion surface collisions, soft-landing is one of those processes. Earlier in this report we showed results that indicate that the soft-landing process is favored at 
collision energies $<50 \mathrm{eV}$. However, at these relatively low energies soft-landing is not the only process taking place, intact scattering of the peptide ion as well as dissociation are among other processes that can compete with the efficiency of soft-landing upon ion surface interactions. The amount of peptide accumulated on the surface is expected to increase with a decrease in the efficiency of other opposing processes. For example, SID studies of these peptides at $30 \mathrm{eV}$ collision energy - same energy at which soft-landing was performed to obtain the plots shown in Figure 2.14 - show a fragmentation efficiency of about $30 \%$ for substance $\mathrm{p}[\mathrm{M}+2 \mathrm{H}]^{2+}$ and about $45 \%$ for bradykinin $[\mathrm{M}+2 \mathrm{H}]^{2+}$. This result indicates that for bradykinin at $30 \mathrm{eV}$ soft-landing/collision energy, peptide ion fragmentation upon ion-surface interaction competes with the softlanding process to a higher extent than in the case of substance $p$. Thus better accumulation of substance $\mathrm{p}$ on the FSAM surface upon soft-landing at $30 \mathrm{eV}$ and earlier saturation as observed in Figure 2.14.

A significant challenge in the study presented here was to accurately determine the amount of material deposited into the surface, mostly because the sputtering yields obtained for soft-landed material were much higher than those obtained for standard solutions of known concentration electrosprayed onto FSAM surfaces, making any comparisons impractical, see Figure 2.15. After the equivalent to $25 \%$ of a monolayer of substance $\mathrm{p}$ was deposited onto an FSAM surface by ion soft-landing, $25 \mathrm{kV} \mathrm{Ga}{ }^{+}$ToFSIMS analysis of the surface revealed the mass spectrum in Figure 2.16a. Figure 2.16b shows a $25 \mathrm{kV} \mathrm{Ga}{ }^{+}$ToF-SIMS analysis of an FSAM surface after deposition of about $25 \%$ of a monolayer of substance $p$ by direct electrospray onto the surface. The peptide ion intensity on the soft-landing mass spectrum is about 50 times higher than the intensity observed upon direct electrospray onto the FSAM surface.

Even though direct quantitation of the peptide ion upon soft-landing could not be attained by SIMS analysis we estimated that we can deposit at least $1 \mathrm{ng}(\leq 1.0 \mathrm{ng})$ of peptide onto the surface. Under the given experimental conditions a monolayer contains about $1 \times 10^{12}$ ions. Upon deposition of $60 \%$ of a monolayer - the point at which saturation occurs for the case of bradykinin - we have successfully deposited at least $1 \mathrm{ng}$ $(\leq 1.0 \mathrm{ng}$ ) of peptide on the surface. Similarly after only $10 \%$ of a monolayer has been deposited the amount of material on the surface is $\leq 170 \mathrm{pg}$.

\section{Ex situ ToF-SIMS Analysis of FSAM Surfaces after Soft-Landing}

Figure 2.16 shows the mass spectrum of a fluorinated self-assembled monolayer after soft-landing of doubly-charged gramicidin s as analyzed ex situ by $\mathrm{Ga}^{+} 15 \mathrm{eV}$ ToFSIMS about 15 minutes after soft-landing on the FTICR. The intact singly-protonated peptide ion of gramicidin s is observed upon ex situ ToF-SIMS analysis, missing in the mass spectrum are peptide fragments normally observed upon in situ $2 \mathrm{keV} \mathrm{Cs}{ }^{+}$analysis on the FTICR. This difference in fragmentation can be attributed to the energy of the primary ions used for in situ and ex situ analysis, $2 \mathrm{keV} \mathrm{Cs}^{+}$and $25 \mathrm{keVGa}^{+}$, respectively. During SIMS analysis the fragmentation observed for sputtered species is known to decrease with primary ion energy. As explained above, at higher primary ion energies, less fragmentation is observed due to desorption of a greater number of low-energy molecular ions than higher-energy ions. ${ }^{[28]}$ This is because the penetration depth of the primary ion increases approximately linearly with collision energy while the affected 
volume increases cubically. ${ }^{[26]}$ In addition, cesium primary ions for SIMS analysis are generated from surface ionization while gallium is a liquid metal ion field emission. Liquid metal ion guns (LMIGs) such as Ga provide small spot sizes $(>30 \mathrm{~nm})$ and high brightness $\left(10^{6} \mathrm{~A} \mathrm{~cm}^{-2} \mathrm{Sr}^{-2}\right)$ which is essential to make a sufficient number of secondary ions available for MS analysis from a small spot. ${ }^{[35]}$ On the other hand surface ionization guns such as $\mathrm{Cs}^{+}$provide slightly larger spot sizes ( $\left.>1 \mathrm{um}\right)$ and much less brightness $\left(500 \mathrm{~A} \mathrm{~cm}^{-2} \mathrm{Sr}^{-2}\right.$ ) than LMICs. Smaller spots and higher brightness during static SIMS analysis o surfaces translates into high amounts of molecules sputtered from the surface with lower internal energies and thus less probability of dissociation. In addition, the time frame of the ToF-SIMS experiment is about 4 orders of magnitude lower than the time frame provided by the SIMS-FTICR experiment. The smaller amount of time for dissociation provided by the ToF-SIMS experiment (ex situ analysis) might also explain the small amount of internal ions observed.

Successful ex situ analysis of the modified surface demonstrates the stability of the soft-landed material on air. The time elapsed between ion deposition by soft-landing and ex situ SIMS analysis was on average 15 minutes, during this period of time the surface was exposed to laboratory air and ambient temperature. The decrease in peptide ion signal as a function of time under vacuum pressure $\left(2.0 \times 10^{9}\right.$ Torr $)$ and under atmospheric pressure $\left(7.6 \times 10^{2}\right.$ Torr $)$ was also investigated, results are shown in Figure 2.17. Under vacuum pressure we observed a decrease on the peptide ion signal of about $30 \%$ over a period of two hours. On the other hand, under atmospheric pressure we observed a decrease on peptide ion signal of about $80 \%$ over the same period of time. If desorption of the peptide from the organic monolayer was taking place then one would expect the opposite result, lower pressures would better assist on a desorption process. If the peptide is not being desorbed from the surface an alternative explanation would be that the ion is being neutralized and thus the ion yields upon SIMS analysis decrease with time. This is consistent with our results since neutralization should be strongly favored upon atmospheric conditions and one would expect lower ion yields. Notice that even though the peptide ion signal decreases significantly under atmospheric pressure it reaches a plateau in which, one can say, that equilibrium of the neutralization reaction has been reached.

In order to gain insights into the nature of the interaction between the soft-landed peptide and the organic monolayer we simply rinsed the surface with EtOH after softlanding, the results are shown in Figure 2.18. Notice that after rinsing of the surface the all the ions corresponding to the soft-landed peptide are missing and those ions that correspond to the surface remain intact on the mass spectrum. This indicates that the interaction between the peptide and the organic monolayer is definitely not covalent in nature but much weaker.

\section{Conclusions}

The ability to selectively deposit ions onto surfaces is a topic of growing interest for its potential applications in technology, material science and much recently preparative mass spectrometry. Here we identified some of the factors that govern and/or affect the soft-landing of peptides into organic monolayers. The energy at which softlanding occurs proved one more time to be a crucial factor for the successful deposition 
of ions onto surfaces. This was also the case for small organic ions soft-landed on FSAM surfaces. However one major difference between small ion and peptide soft-landing is that no "crash landing" was observed for the case of peptides.

Significant differences in the amount of peptide sputtered after soft-landing indicated that the peptides studied here have distinct interactions with the organic monolayer. The results also suggest that the inert nature of the surface is responsible for the retention of charge of the soft-landed peptide. The retention of charge of the peptide is very significant in that ionization of the peptide is not necessary to successfully sputtered ions from the surface. This phenomenon was found to dramatically increase the ion yield normally obtained upon SIMS analysis.

It was also determined that soft-landed peptides can remain embedded into selfassembled monolayers for long periods of time without significant desorption of the ion into the gas-phase. In addition, the amount of peptide deposited into the surface can be controlled by choice of total ion dose, the projectile and its energy. The interactions between the peptide ion and the surface are such that the material deposited on a FSAM surface can be easily wash out from the surface. This alludes to the non-destructive nature of the soft-landing process, something not normally attributed to mass spectrometric techniques.

\section{Acknowledgments}

Jormarie Alvarez acknowledges participation in Pacific Northwest National Laboratory Interfacial and Condensed Phase Summer Research Institute. We acknowledge helpful discussions with Stephan E. Barlow. Thanks to Daniel Gaspar for SIMS experiments. 


\section{List of References}

[1] V. Franchetti, B. H. Solka, W. E. Baitinger, J. W. Amy, R. G. Cooks, Int. J. Mass Spectrom. Ion Physics 1977, 23, 29.

[2] S. A. Miller, H. Luo, S. Pachuta, R. G. Cooks, Science 1997, 275, 1447.

[3] R. J. Geiger, M. C. Melnyk, K. L. Busch, M. G. Bartlett, Int. J. Mass Spectrom. 1999, $183,415$.

[4] M. A. LaPack, S. J. Pachuta, K. L. Busch, R. G. Cooks, Int. J. Mass Spectrom. Ion Phys. 1983, 53, 323.

[5] Z. Ouyang, Z. Takats, T. A. Blake, B. Gologan, A. J. Guymon, J. M. Wiseman, J. C. Oliver, V. J. Davisson, R. G. Cooks, Science 2003, 301, 1351.

[6] B. Feng, D. S. Wunschel, C. D. Masselon, L. Pasa-Tolic, R. D. Smith, J. Am. Chem. Soc. 1999, 121, 8961.

[7] G. Siuzdak, B. Bothner, Angew. Chem. Int. Ed. 1995, 34, 2053.

[8] S. D. Fuerstenau, W. H. Benner, J. J. Thomas, C. Brugidou, B. Bothner, G. Siuzdak, Angew. Chem. Int. Ed. 2001, 40, 542.

[9] M. R. Morris, D. E. J. Riederer, B. E. Winger, R. G. Cooks, T. Ast, C. E. D. Chidsey, Int. J. Mass Spectrom. Ion Processes 1992, 122, 181.

[10] R. G. Cooks, T. Ast, M. A. Mabud, Int. J. Mass Spectrom. Ion Processes 1990, $100,209$.

[11] D. G. Schultz, H. Lim, S. Garbis, L. Hanley, J. Mass Spectrom. 1999, 34, 217.

[12] J. Laskin, J. H. Futrell, J. Chem. Phys. 2002, 116, 4302.

[13] B. Willerding, W. Heiland, K. J. Snowdon, Phys. Rev. Lett. 1984, 53, 2031.

[14] A. Somogyi, T. E. Kane, J. M. Ding, V. H. Wysocki, J. Am. Chem. Soc. 1993, 115,5275 .

[15] H. Luo, S. Miller, R. G. Cooks, S. Pachuta, Int. J. Mass Spectrom. Ion Processes 1998, 174, 193.

[16] J. Shen, Y. H. Yim, B. Feng, V. Grill, C. Evans, R. G. Cooks, Int. J. Mass Spectrom. 1999, 182/183, 423. 
[17] B. Gologan, Z. Takáts, J. Alvarez, J. M. Wiseman, N. Talaty, Z. Ouyang, R. G. Cooks, J. Am. Soc. Mass Spectrom. 2004, in press.

[18] J. Laskin, E. V. Denisov, A. K. Shukla, S. E. Barlow, J. H. Futrell, Anal. Chem. 2002, 74, 3255 .

[19] M. W. Senko, J. D. Canterbury, S. Guan, A. G. Marshall, Rapid Commun. Mass Spectrom. 1996, 10, 1839.

[20] M. J. Tarlov, J. G. Newman, Langmuir 1992, 8, 1398.

[21] P. Roepstorff, J. Fohlmann, Biomed. Mass Spectrom. 1984, 11, 601.

[22] K. Biemann, Methods Enzymol. 1990, 193, 886.

[23] T. Wyttenbach, G. V. Helden, M. T. Bowers, J. Am. Chem. Soc. 1996, 18, 8355.

[24] A. C. Gill, K. R. Jennings, T. Wyttenbach, M. T. Bowers, Int. J. Mass Spectrom. 2000, 195-196, 685 .

[25] D. J. Butcher, K. G. Asano, D. E. Goeringer, S. M. McLuckey, J. Phys. Chem. A 1999, 103, 8664

[26] B. E. Winger, O. W. Hand, R. G. Cooks, Int. J. Mass Spectrom. Ion Processes 1988, $84,89$.

[27] J. Amster, J. A. Loo, J. J. P. Furlong, F. W. McLafferty, Anal. Chem. 1987, 59, 313.

[28] M. Barber, J. C. Vickerman, J. Wolstenholme, J. Chem. Soc. Faraday Trans. I 1980, 76, 549.

[29] W. Ens, R. Beavis, D. Main, X. Tang, B. T. Chait, in 34th ASMS Conference on Mass Spectrometry and Allied Topics, Cincinnati, 1986.

[30] C. E. D. Chidsey, D. M. Loiacono, Langmuir 1990, 6, 682.

[31] Y. F. Miura, M. Takenaga, T. Koini, M. Graupe, N. Garg, R. L. Graham Jr., T. R. Lee, Langmuir 1998, 14, 5821.

[32] G. M. Whitesides, P. E. Laibinis, Langmuir 1990, 6, 87.

[33] N. P. Ewing, G. A. Pallante, X. Zhang, C. J. Cassady, J. Mass. Spectrom. 2001, 36,875 .

[34] S. R. Carr, C. J. Cassady, J. Mass. Spectrom. 1997, 32, 959. 
[35] L. Van Vaeck, A. Adriaens, R. Gijbels, Mass Spectrom. Rev. 1999, 18, 1.

Table 2.1 Peptide fragments obtained upon $\mathrm{Cs}^{+}$sputtering analysis at various kinetic energies of an FSAM surface modified with bradykinin $[\mathrm{M}+2 \mathrm{H}]^{2+}$.

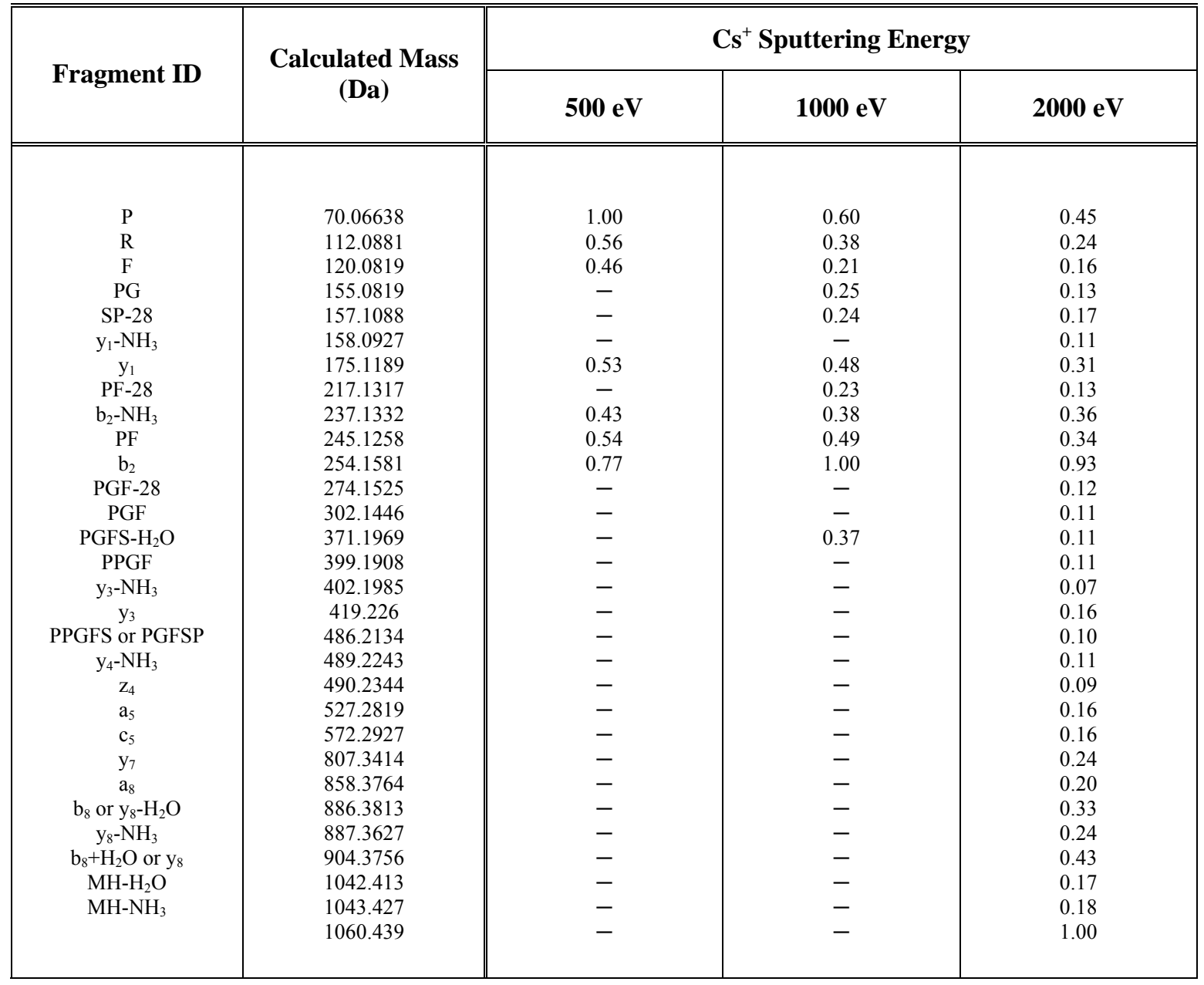


Table 2.2 Relative amount of peptide sputtered from fluorinated organic monolayers (FSAMs) after $30 \mathrm{eV}$ soft-landing.

\begin{tabular}{|c|c|c|c|c|c|}
\hline Peptide & $\begin{array}{c}{[M+H]^{+} \text {Mass }} \\
(D a)\end{array}$ & $n$ & $\begin{array}{l}\text { Charge } \\
\text { State }\end{array}$ & $\begin{array}{c}\text { Relative Amount } \\
\text { of }[M+H]^{+} \text {Sputtered }\end{array}$ & AA Sequence \\
\hline Gramicidin S & 1141 & 0 & 2 & 1.16 & LFPVOLFPVO \\
\hline RVYIFPF & 941.5 & 2 & 1 & 1.07 & RVYIFPF \\
\hline Substance P & 1347.7 & 3 & 2 & 1.00 & RPKPQQFFGLM \\
\hline $\begin{array}{c}\text { (Angiotensin III) } \\
\text { RVYIHPF }\end{array}$ & 931.5 & 2 & 1 & 0.96 & RVYIHPF \\
\hline Leucine Enkephalin & 556.2 & 1 & 1 & 0.90 & YGGFL \\
\hline des-Arg ${ }^{9}$ Bradykinin & 904.4 & 2 & 1 & 0.84 & RPPGFSPF \\
\hline des-Arg ${ }^{1}$ Bradykinin & 904.4 & 2 & 1 & 0.63 & PPGFSPFR \\
\hline Bradykinin & 1060.5 & 3 & 2 & 0.40 & RPPGFSPFR \\
\hline $\begin{array}{l}\text { Renin Substrate } \\
\text { Porcine }\end{array}$ & 1758.9 & 2 & 3 & 0.15 & DRVYIHPFHLLVYS \\
\hline
\end{tabular}

$\mathrm{n}$ : number of free amino groups. Include amino groups found at the $\mathrm{N}$-terminus or in the side chain of basic amino acids Arginine (R) and Lysine (K). 


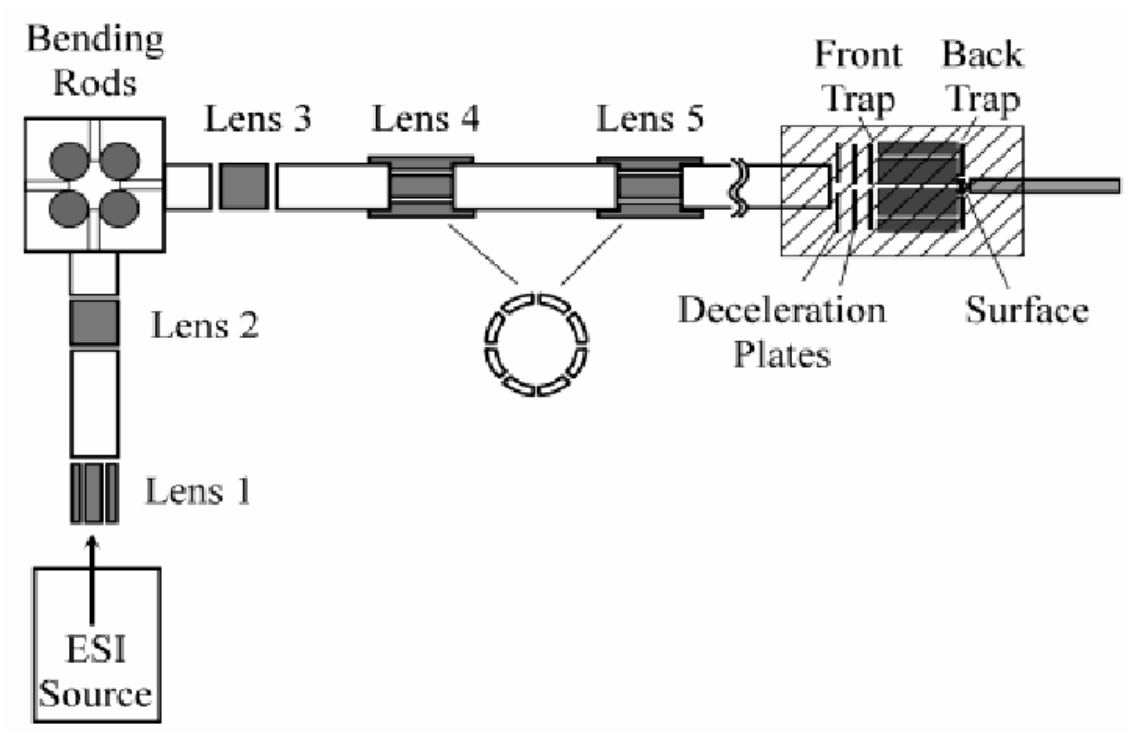

Figure 2.1 Schematic view of Fourier Transform Ion Cyclotron Mass Spectrometer available at Pacific Northwest National Laboratory.

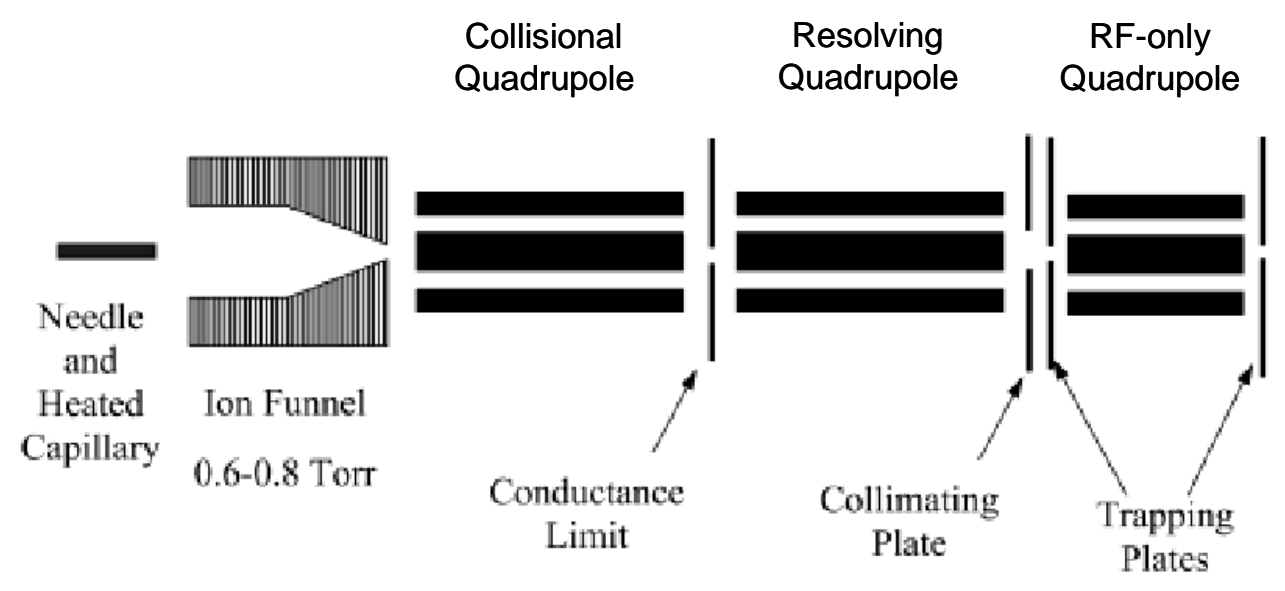

Figure 2.2 Schematic view of the electrospray source of the Fourier Transform Ion Cyclotron Mass Spectrometer available at Pacific Northwest National Laboratory. 

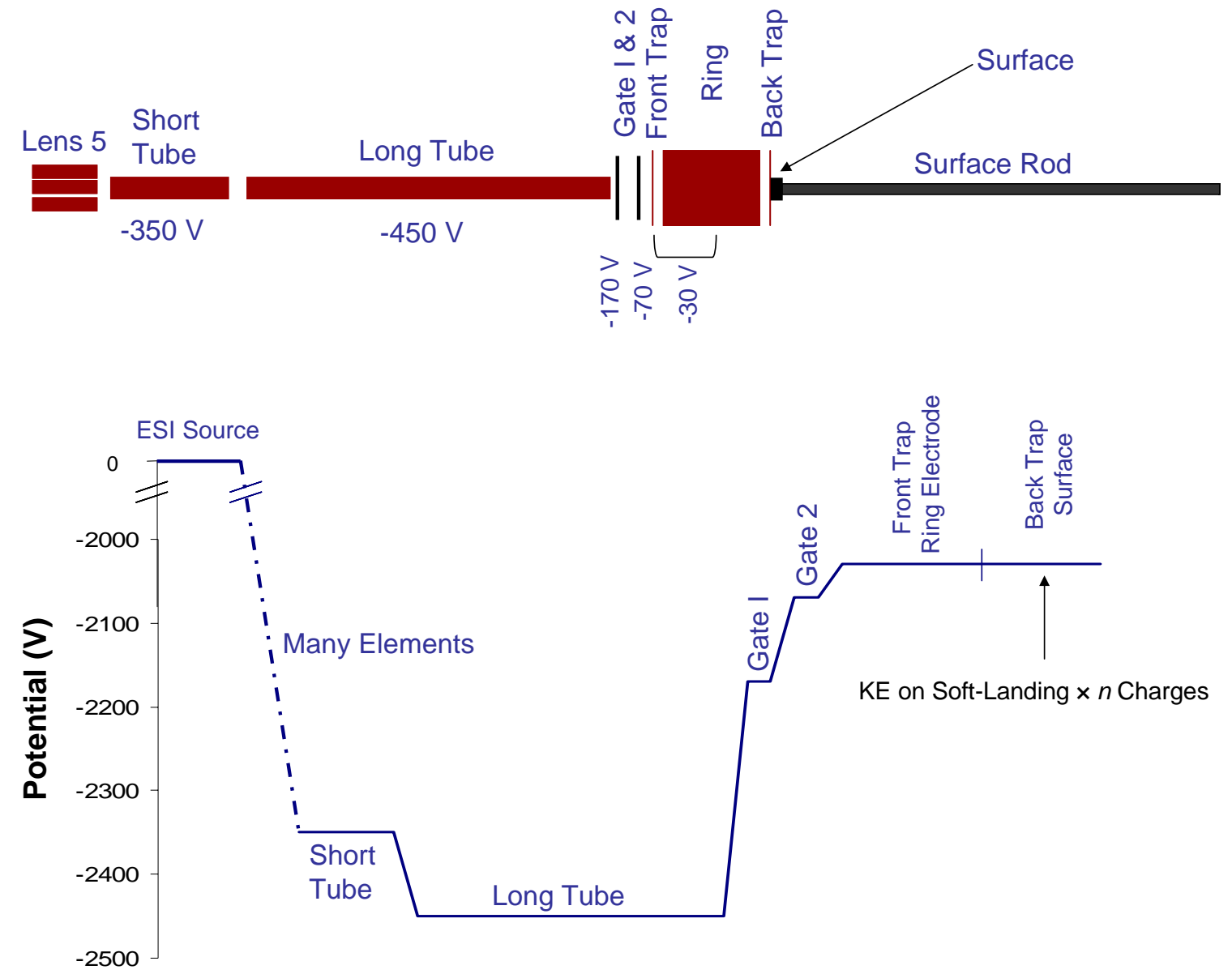

Figure 2.3 Potential diagram for the soft-landing mode in the FTICR mass spectrometer. Voltages shown are relative to the ESI source at ground potential. 

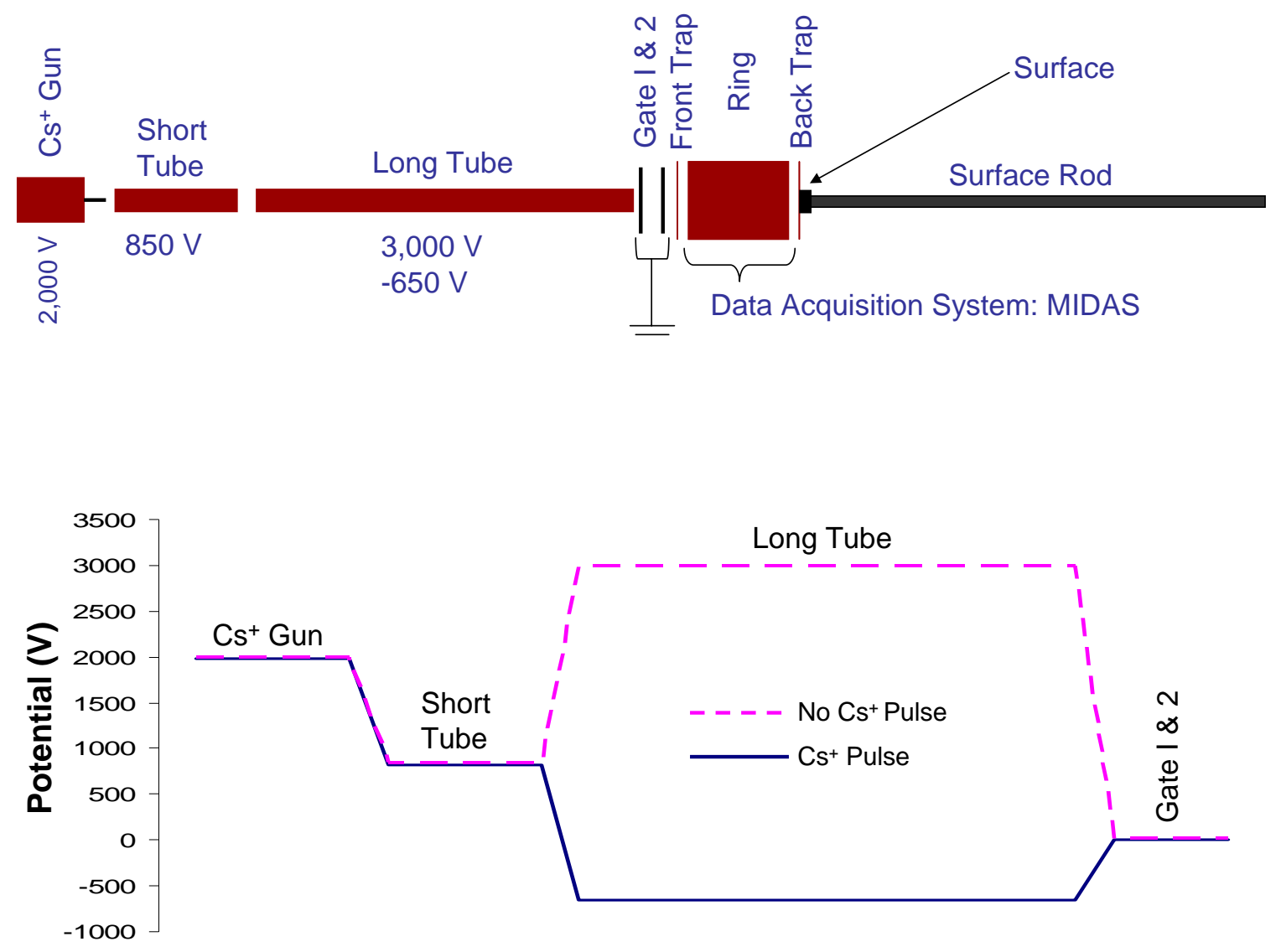

Figure 2.5 Potential diagram of the SIMS analysis mode in the FTICR mass spectrometer. 


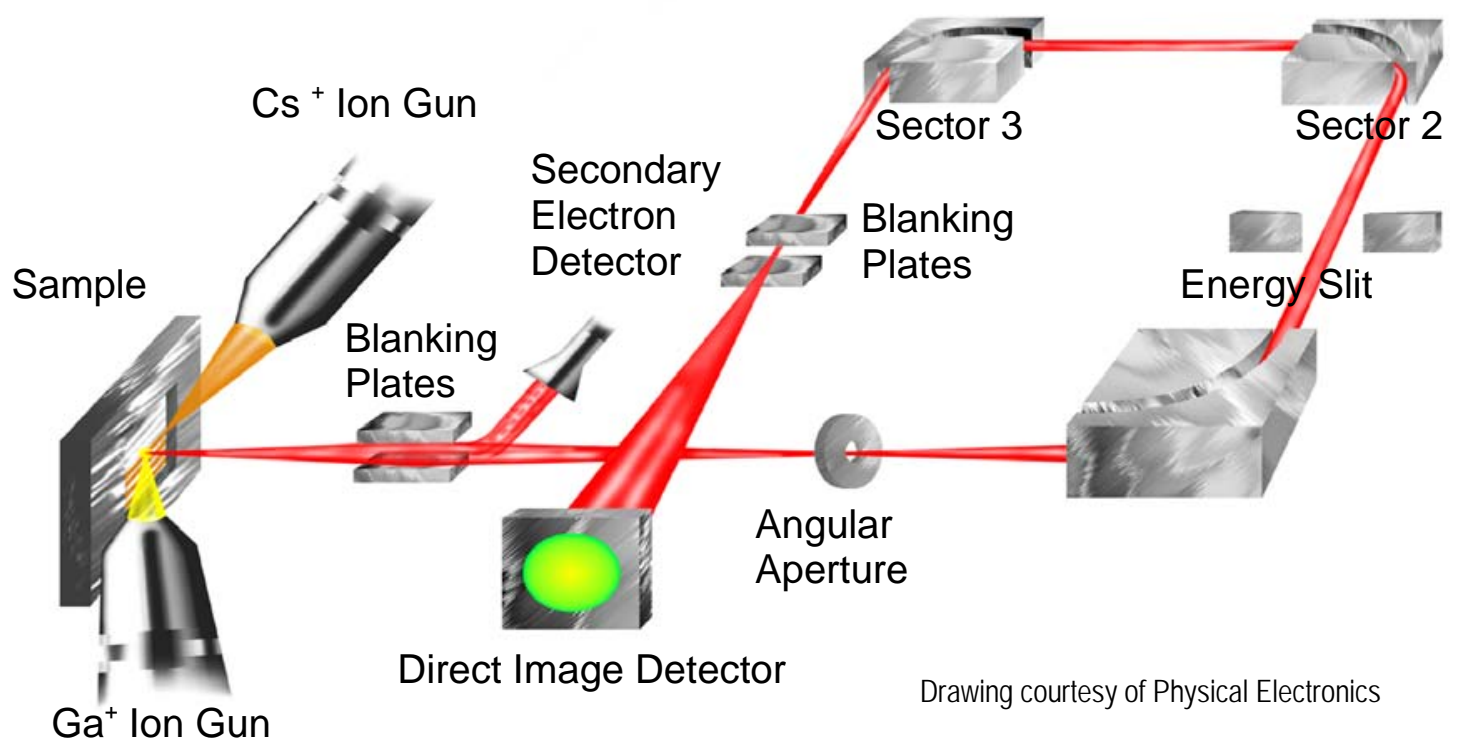

Figure 2.6 PHI TRIFT time of flight-secondary son mass spectrometer at the Pacific Northwest National Laboratory.

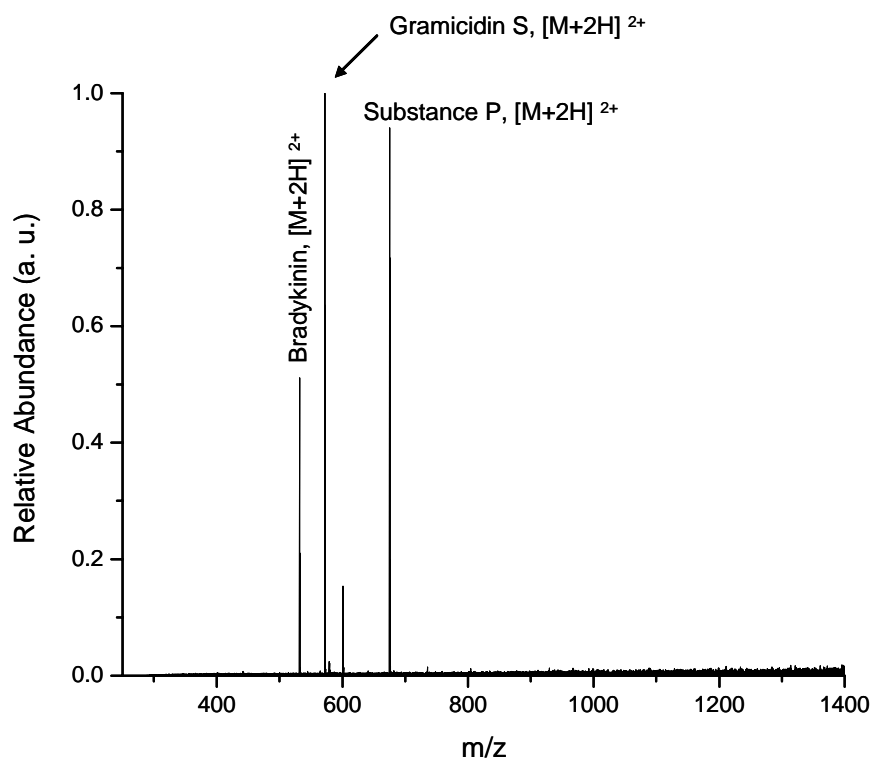

Figure 2.7 ESI-FTICR mass spectrum of a tertiary mixture solution with a concentration of $0.1 \mathrm{mg} / \mathrm{mL}$ in each peptide: bradykinin, gramicidin $\mathrm{s}$ and substance p. Samples were dissolved in a 70:30 (v/v) methanol:water solution with $1 \%$ acetic acid. ESI conditions: flow rate of $0.3 \mathrm{~mL} / \mathrm{min}$ and spray voltage of $2 \mathrm{kV}$. 


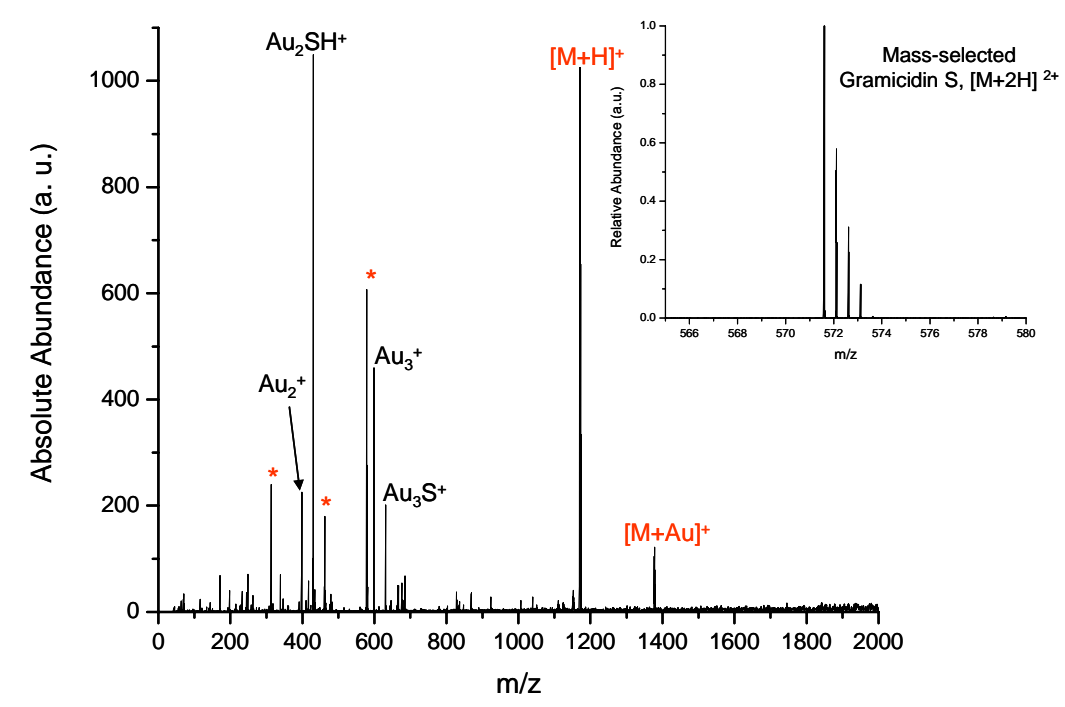

Figure 2.8 SIMS-FTICR $\left(2 \mathrm{keV} \mathrm{Cs}{ }^{+}\right)$mass spectrum of doubly-charged $[\mathrm{M}+2 \mathrm{H}]^{2+}$ gramicidin s deposited onto an FSAM surface after mass selection from a mixture solution. Peptide fragment ions are labeled with $(*)$. The insert shows the isolation of the isotopic distribution of doubly-charged gramicidin $\mathrm{s}$ with a $4 \mathrm{Th}$ window width.

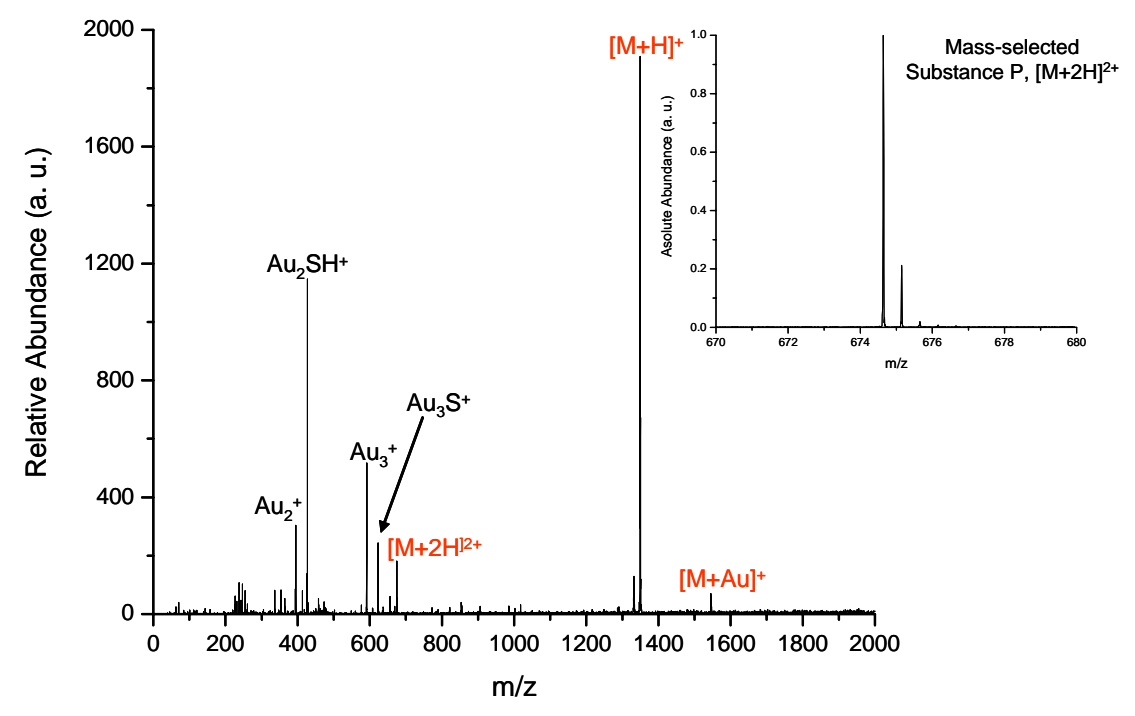

Figure 2.9 SIMS-FTICR $\left(2 \mathrm{keV} \mathrm{Cs}{ }^{+}\right)$mass spectrum of doubly-charged $[\mathrm{M}+2 \mathrm{H}]^{2+}$ substance $\mathrm{p}$ deposited onto an FSAM surface after mass selection from a mixture solution. The insert shows the isolation of the isotopic distribution of doubly-charged substance $\mathrm{p}$ with a 4 Th window width. 


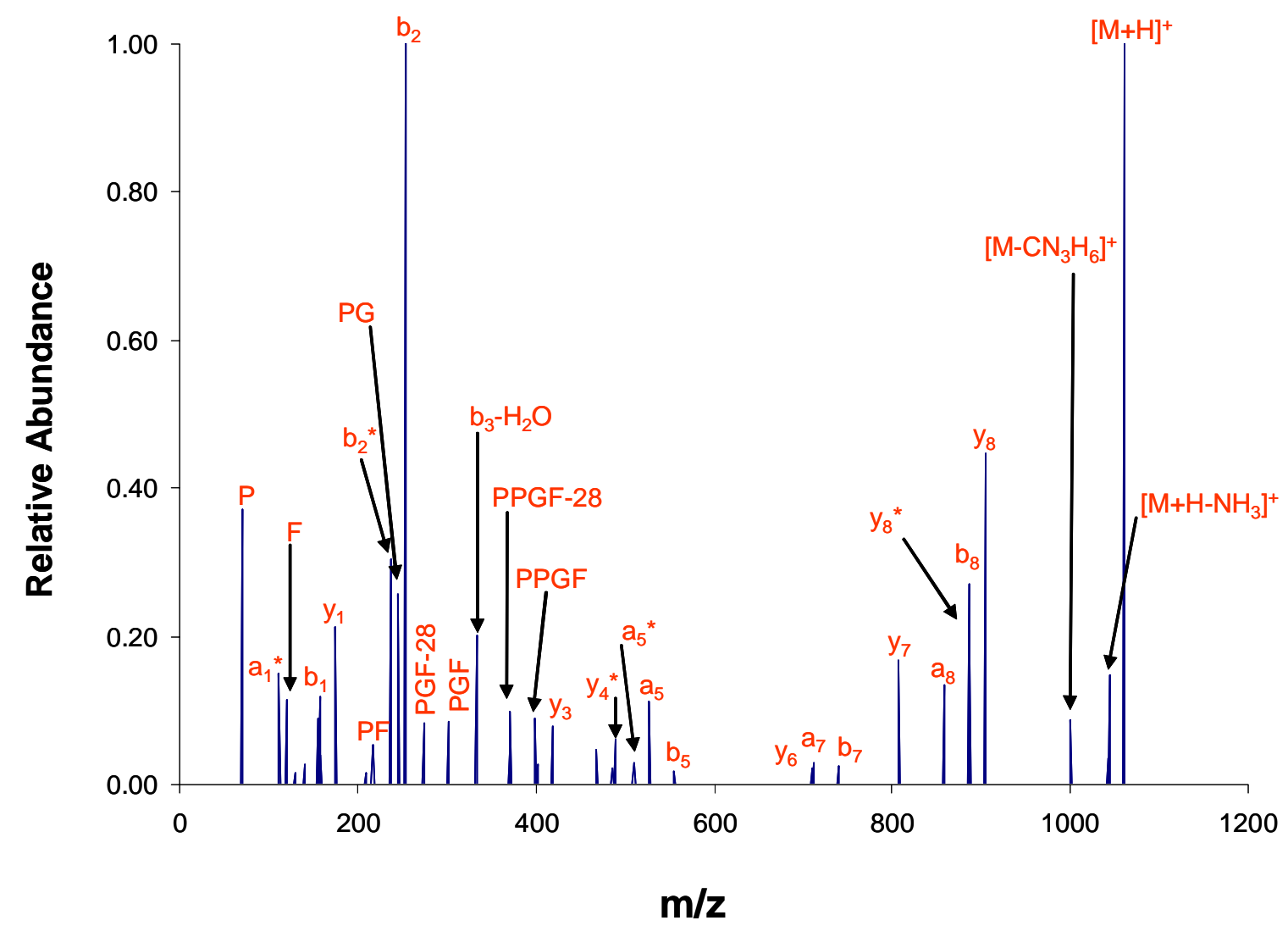

Figure 2.10 SIMS-FTICR $\left(2 \mathrm{keV} \mathrm{Cs}{ }^{+}\right)$mass spectrum of doubly-charged $[\mathrm{M}+2 \mathrm{H}]^{2+}$, $\mathrm{m} / \mathrm{z}$ 530, bradykinin deposited onto an FSAM surface. All the peaks shown are fragment ions of the peptide. The peaks characteristic of the surface are omitted for simplicity. 


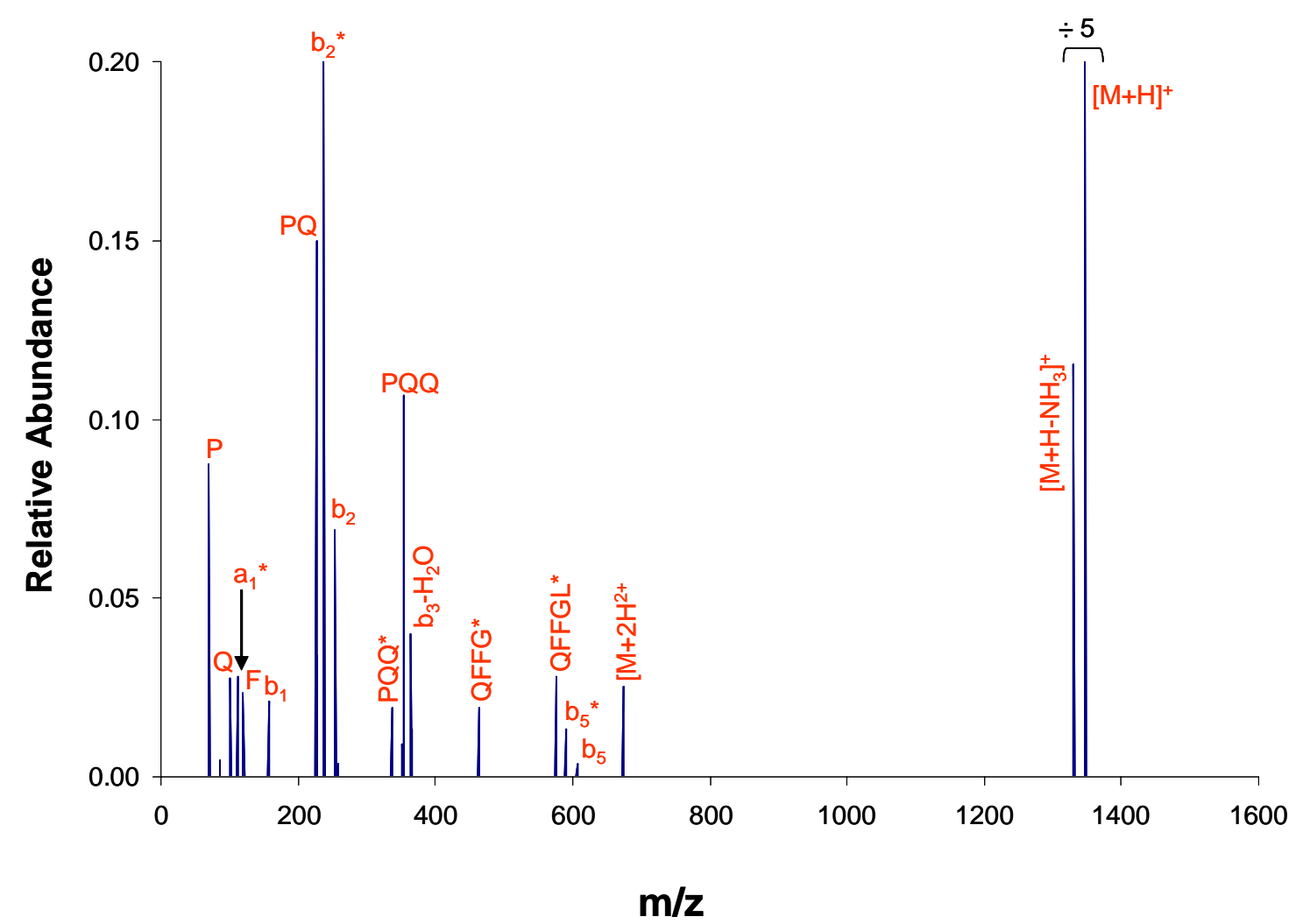

Figure 2.11 SIMS-FTICR $\left(2 \mathrm{keV} \mathrm{Cs}^{+}\right)$mass spectrum of doubly-charged $[\mathrm{M}+2 \mathrm{H}]^{2+}$ substance $\mathrm{p}$ deposited onto an FSAM surface. All the peaks shown are fragment ions of the peptide. The peaks characteristic of the surface are omitted for simplicity.
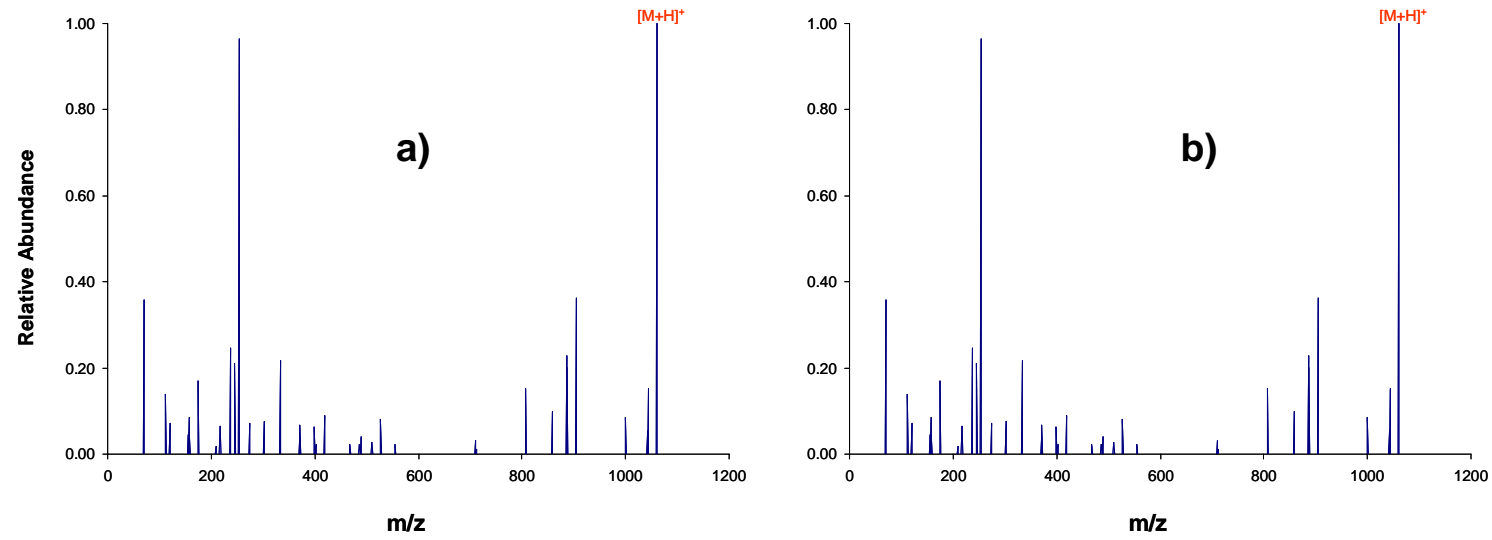

Figure 2.12 SIMS-FTICR $\left(2 \mathrm{kV} \mathrm{Cs}^{+}\right)$mass spectrum doubly-charged $[\mathrm{M}+2 \mathrm{H}]^{2+}$ bradykinin deposited onto an FSAM surface at a) $20 \mathrm{eV}$ and b) $150 \mathrm{eV}$ soft-landing energy. All the peaks shown are fragment ions of the peptide. The peaks characteristic of the surface are omitted for simplicity. 

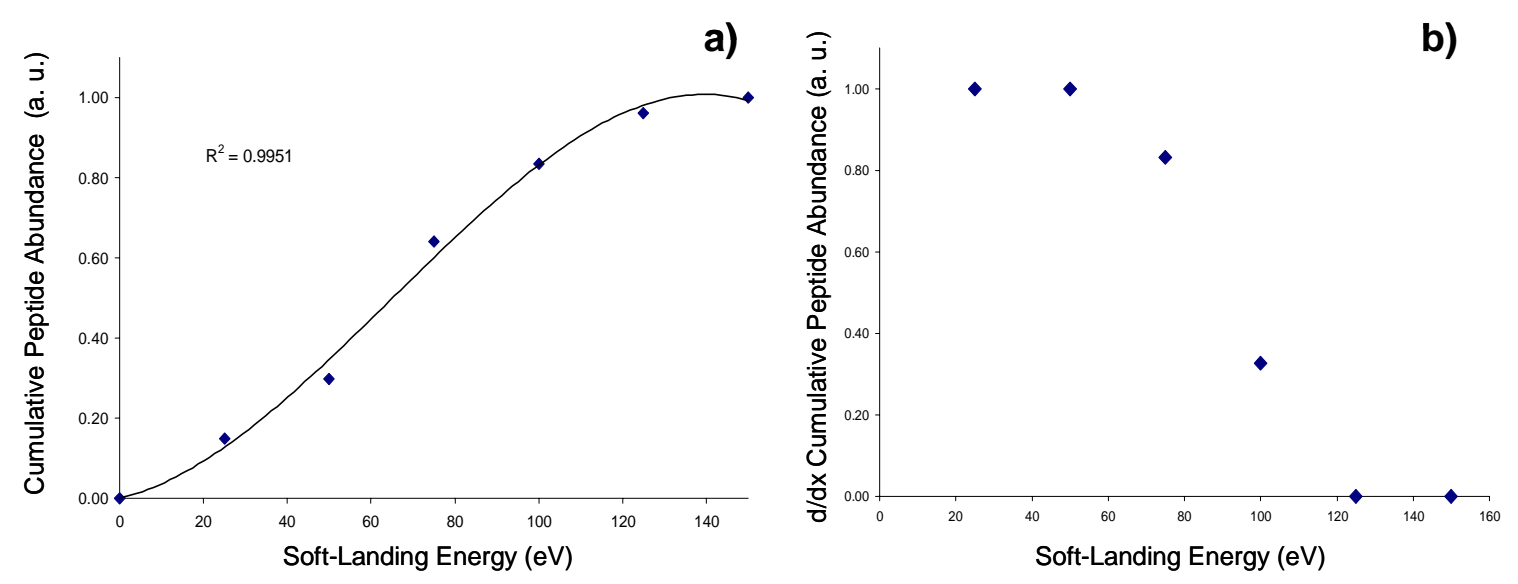

Figure 2.13 a) Cumulative peptide ion abundance (substance $p$ ) as a function of softlanding energy. b) First derivative of the plot shown in a.
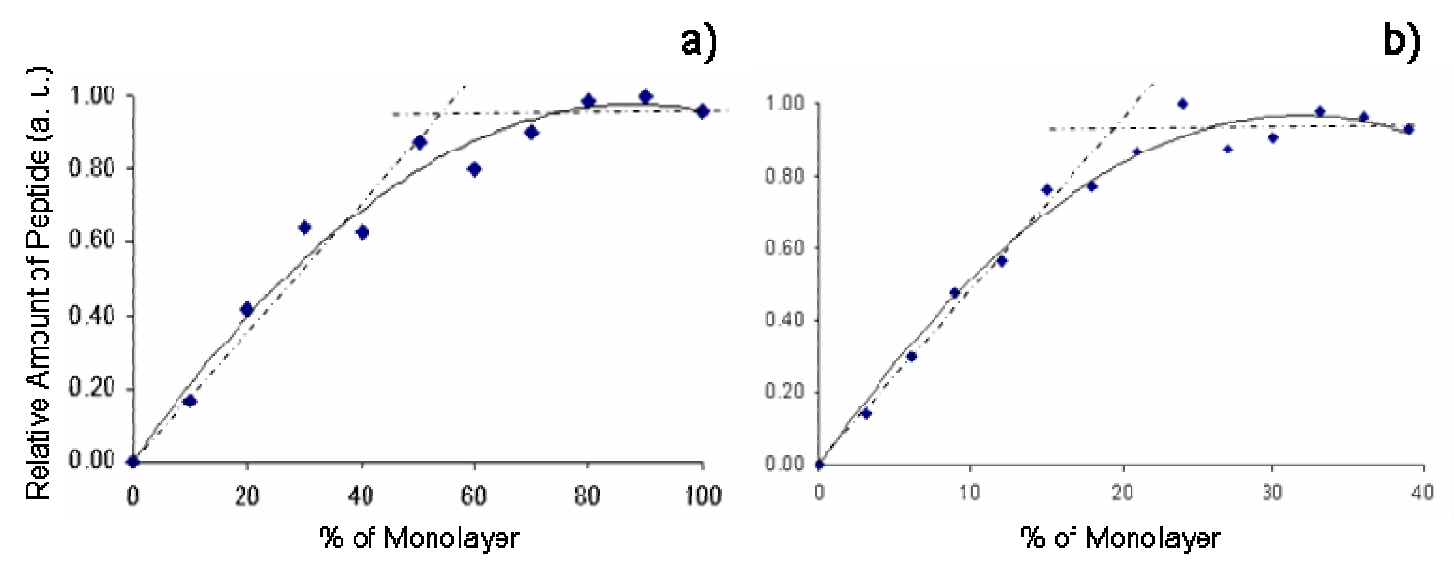

Figure 2.14 Saturation plots showing the relative amount of peptide ion sputtered from the surface as a function of amount of monolayer deposited of a) bradykinin and b) substance $p$. 
a)
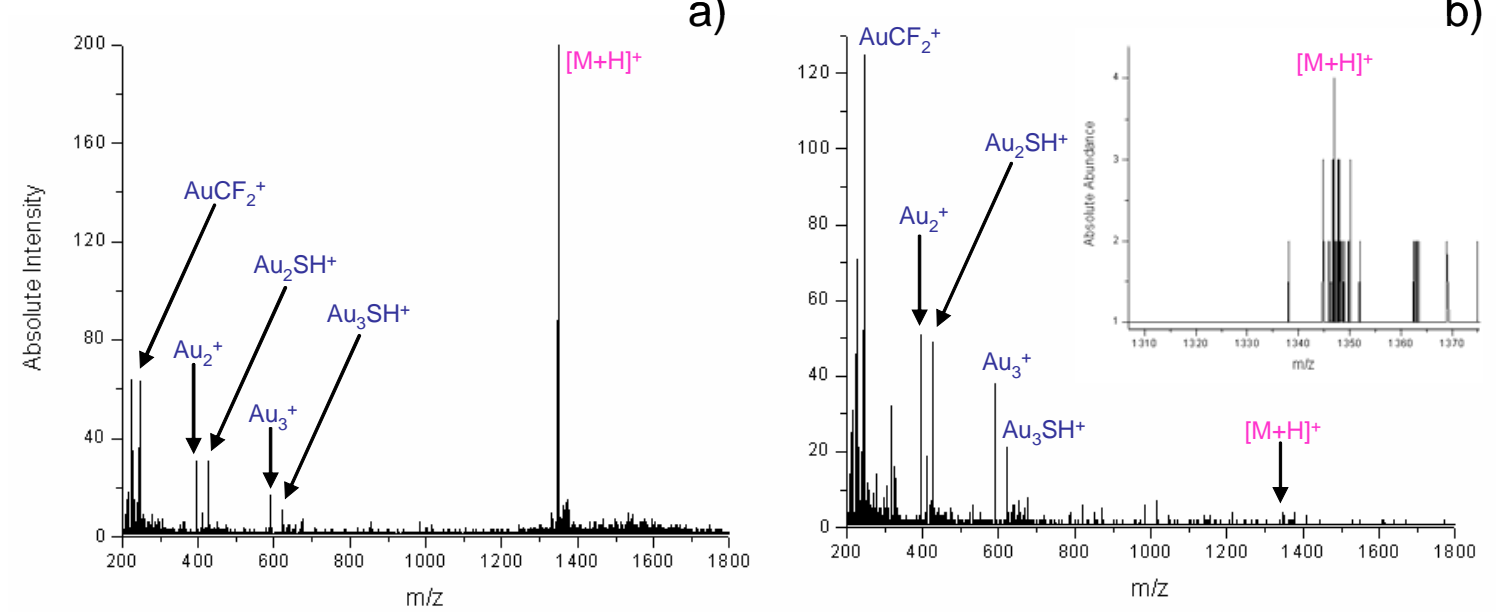

Figure 2.15 ToF-SIMS $\left(25 \mathrm{keV} \mathrm{Cs}{ }^{+}\right)$mass spectrum of FSAM surface modified with substance $\mathrm{p}$ by a) ion soft-landing at $30 \mathrm{eV}$ and b) direct electrospray of the equivalent to $25 \%$ of a monolayer.
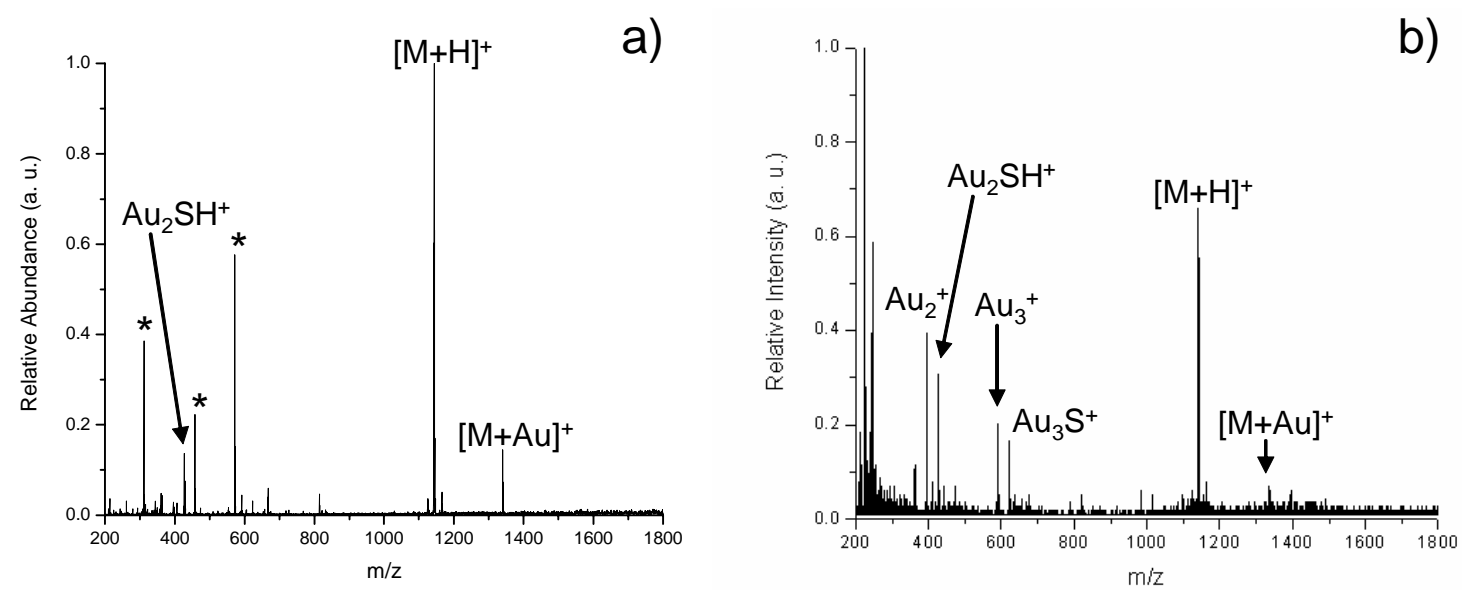

Figure 2.16 Mass spectrum gramicidin s deposited onto an FSAM surface as analyzed by a) $2 \mathrm{keV} \mathrm{Cs}^{+}$SIMS-FTICR and b) $25 \mathrm{keV} \mathrm{Ga}^{+}$ToF-SIMS. Peptide fragment ions are labeled with $(*)$. 
a)

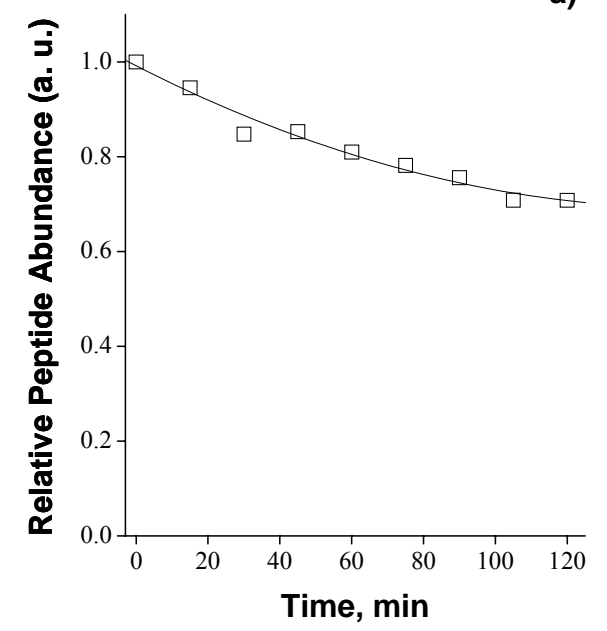

b)

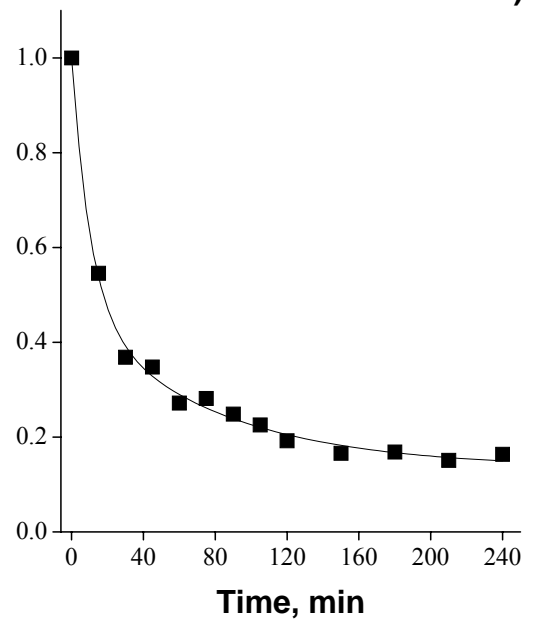

Figure 2.17 Loss of peptide signal as a function of time a) under vacuum pressure (2.0 $\times 10^{9}$ Torr) and b) under atmospheric conditions $\left(7.6 \times 10^{2}\right.$ Torr $)$ after soft-landing on FSAM surface. SIMS analysis of soft-landed substance $p$ at $30 \mathrm{eV}$.
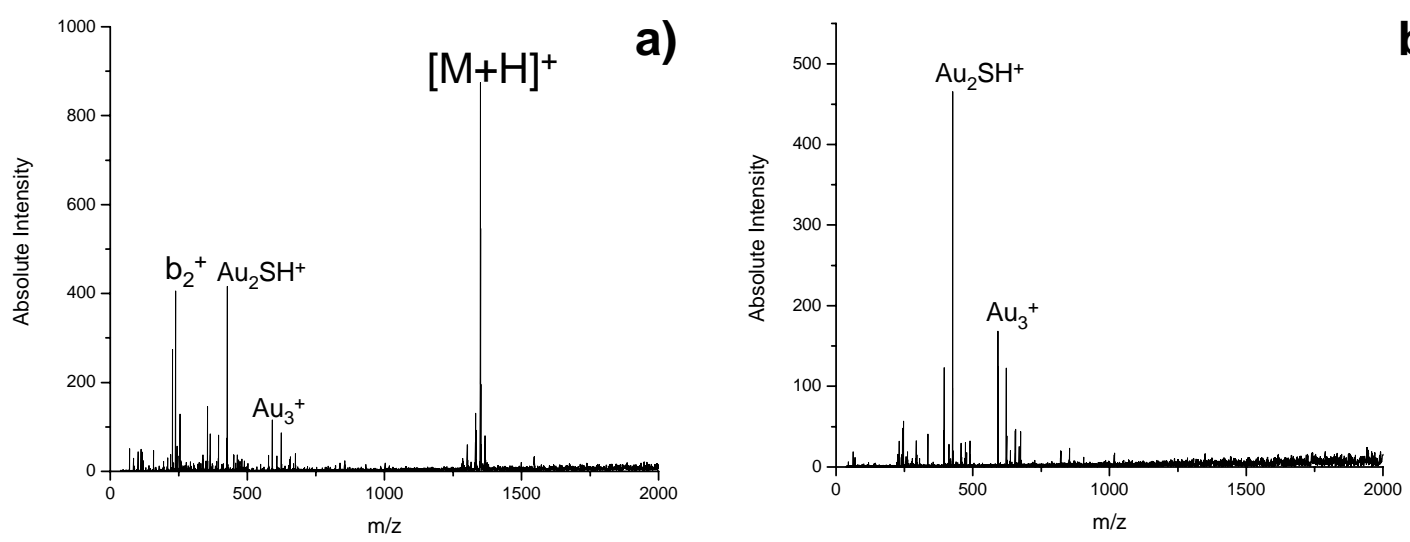

b)

Figure 2.18 Mass spectrum of substance $\mathrm{p}$ soft-landed on FSAM a) before and $\mathrm{b}$ ) after rinsing the surface with ethanol. 


\title{
Growth and Characterization of Highly Oriented Pure and $\mathrm{Gd}_{2} \mathrm{O}_{3}$ doped $\mathrm{CeO}_{2}(111)$ on $\mathrm{ZrO}_{2}(111) / \mathrm{Al}_{2} \mathrm{O}_{3}(0001)$ system
}

\author{
Debasis Bera and S. Seal \\ Materials Science and Engineering \\ University of Central Florida \\ Orlando, FL \\ And \\ Donald Baer \\ Pacific Northwest National Laboratory \\ Richland, WA
}

Aim of the Work: Electrolyte materials with exclusive oxygen ion-conductivity are being considered for modern solid-state fuel cell devices, such as sensors, solid oxide fuel cells, batteries and three-way catalysts of automobiles. Yttria-stabilized zirconia (YSZ), a potential candidate, exhibits adequate oxygen-ion conductivity only at higher temperature $(0.1 \mathrm{~S} / \mathrm{cm}$ at $1275 \mathrm{~K})$. In order to improve the efficiency of solid-state electrochemical devices at lower temperature, a new electrolyte material is needed with higher oxygen ionic conductivity. A considerable attention has been given on the research of epitaxial growth of $\mathrm{CeO}_{2}$ on insulator substrates in the recent years for such technologically important applications. Keeping such application in view, highly oriented pure and $\mathrm{Gd}_{2} \mathrm{O}_{3}$ doped ceria films have been grown on pure and $\mathrm{ZrO}_{2}(111)$ buffered $\mathrm{Al}_{2} \mathrm{O}_{3}(0001)$ substrates using oxygen plasma-assisted molecular beam epitaxy (OPA-MBE). A set of multilayer nanofilms with various doping percentage of $\mathrm{Gd}_{2} \mathrm{O}_{3}$ in ceria was synthesized using OPA-MBE. The growth of these nanofilms was monitored using in-situ reflection high energy electron diffraction (RHEED). An in-situ x-ray photoelectron spectroscopy (XPS) was carried out to monitor the chemical state information of $\mathrm{Gd}_{2} \mathrm{O}_{3}$ doped ceria samples. In addition, these films were characterized by several ex-situ surface and bulk sensitive capabilities including x-ray diffraction (XRD), Rutherford backscattering spectrometry (RBS) and atomic force microscopy (AFM). Inter-diffusion of elements at the $\mathrm{CeO}_{2} / \mathrm{ZrO}_{2}$ interface has been studied using high resolution transmission electron microscopy (HRTEM) and x-ray photoelectron spectroscopy (XPS) depth profiling. The $150 \mathrm{~nm}$ of $\mathrm{CeO}_{2}$ films grown on pure $\mathrm{Al}_{2} \mathrm{O}_{3}(0001)$ substrate show polycrystalline features due to structural deformations resulting from the large lattice mismatch between the $\mathrm{Al}_{2} \mathrm{O}_{3}(0001)$ substrate and the ceria film. However, the ceria films grown on a $3 \mathrm{~nm}$ layer of $\mathrm{ZrO}_{2}(111)$, which is grown as a buffer layer on top of $\mathrm{Al}_{2} \mathrm{O}_{3}(0001)$, appears to be epitaxial and highly oriented. This is presumably due to the much smaller lattice mismatch between cubic zirconia and ceria compared to the substantial differences between the lattice parameters of $\mathrm{Al}_{2} \mathrm{O}_{3}(0001)$ and ceria. Oxygen ionic conductivity in $\mathrm{Gd}_{2} \mathrm{O}_{3}$ doped ceria films has been measured as a function of $\mathrm{Gd}$ concentration and these results will be compared with the ion conductance of the other films.

Deposition of Multilayer Nanofilms: Growth of nanofilms and insitu characterizations were carried out in a dual chamber ultrahigh

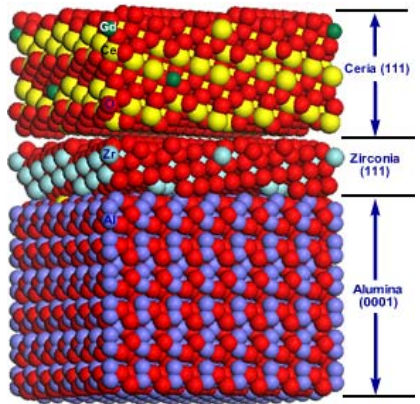


vacuum (UHV) system. The MBE chamber consists of three metal evaporation sources and an UHV compatible electron cyclotron resonance (ECR) oxygen plasma source as well as RHEED for real time characterization of epitaxial growth. High purity $\mathrm{Ce}$ and $\mathrm{Zr}$ rod were used as the source materials in separate electron beam evaporators. Gd was evaporated from effusion cell. Growth rates of these films were monitored by means of quartz crystal oscillators. Polished $\mathrm{Al}_{2} \mathrm{O}_{3}(0001)$ single crystal were used for epitaxial growth zirconia buffered $\mathrm{Gd}_{2} \mathrm{O}_{3}$-doped $\mathrm{CeO}_{2}$ as shown in Fig.1. After introducing the substrate into the MBE chamber, it was cleaned once more at a temperature of $875 \mathrm{~K}$ for $10 \mathrm{~min}$ by exposing activated oxygen plasma from ECR plasma source at an oxygen pressure of $1.5 \times 10^{-5}$ Torr. During growth of multilayer nanofilms, temperature and oxygen partial pressure were optimized to $925 \mathrm{~K}$ and $2.0 \times 10^{-5}$ Torr. $\mathrm{ZrO}_{2}$ layer was deposited at a rate of $0.02 \mathrm{~nm} / \mathrm{s}$. Deposition rate of $\mathrm{CeO}_{2}$ was varied keeping $\mathrm{Gd}_{2} \mathrm{O}_{3}$ constant deposition rate at $0.01 \mathrm{~nm} / \mathrm{s}$ to synthesize $6 \%, 9 \%, 12 \%, 15 \%, 18 \%, 21 \%$ and 24 $\% \mathrm{Gd}_{2} \mathrm{O}_{3}$ doped $\mathrm{CeO}_{2}$. Fig. 1 represents a schematic diagram of the all three layers of nanofilm.

Results: The XRD data in Fig. 2 reveals that the orientation of zirconia on alumina substrate is preferred in one particular direction. The peak positions corresponding to the $\mathrm{Gd}_{2} \mathrm{O}_{3}$-doped $\mathrm{CeO}_{2}$ and $\mathrm{ZrO}_{2}$ in the spectrum also matched the reference data from the cubic fluorite $\mathrm{CeO}_{2}$ and $\mathrm{ZrO}_{2}$ structure.

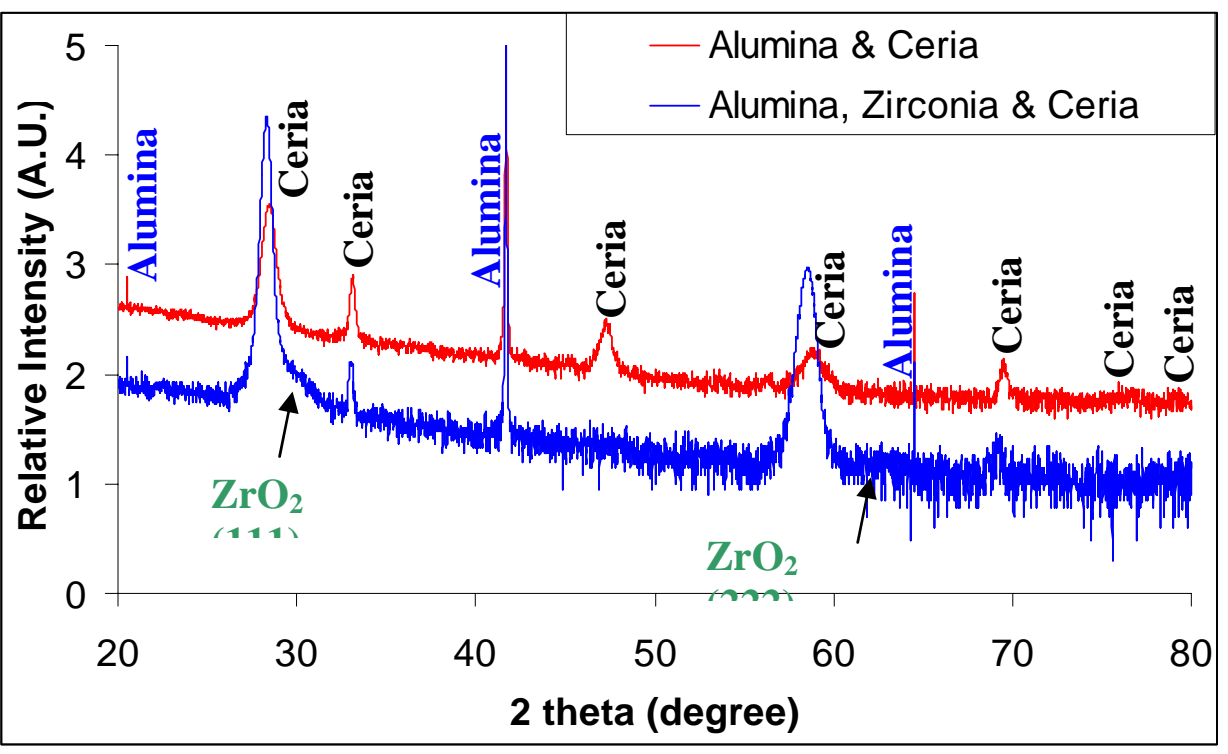

Fig. 2: The XRD data for heterolayers of (a) Gd2O3-doped ceria on the alumina (0001) [red line], (b) Gd2O3-doped ceria on the zirconia buffered alumina (0001) substrate. Extra peak of ceria in (a) shows polycrystalline

RBS and channeling measurements were carried out at room temperature using $2.04 \mathrm{MeV} \mathrm{He}{ }^{+}$ions at normal incidences. In Fig.3, a RBS spectrum is shown along with simulated results using SIMNRA software. The simulated results agreed well with experimental data. The simulated results of ceria samples doped with different percentage of $\mathrm{Gd}_{2} \mathrm{O}_{3}$ show that the thickness of ceria and zirconia are to be approximately $150 \mathrm{~nm}$ and $3 \mathrm{~nm}$. The atomic concentrations of $\mathrm{Gd}$ of different samples were same as the calculated values from QCO. The electrical conductivity of these films was measured using a four-probe Van der Pauw technique. Oxygen ionic conductivity results from samples with different concentration of $\mathrm{Gd} 2 \mathrm{O} 3$ have been presented in Fig. 4. For 
comparison the oxygen ionic conductivity data from polycrystalline and single crystal yttria stabilized zirconia are also shown in the figure. In general, the higher conductivities compared to the bulk polycrystalline YSZ and single crystal YSZ. The ionic conductivity data from three samples, 3, 4 and 5, have shown in Fig 4. Atomic concentration of Gd in samples 3, 4 and 5 are 12 , 6and $24 \%$. Detailed investigations are underway to study the influence of doping concentration on ionic conductivity.

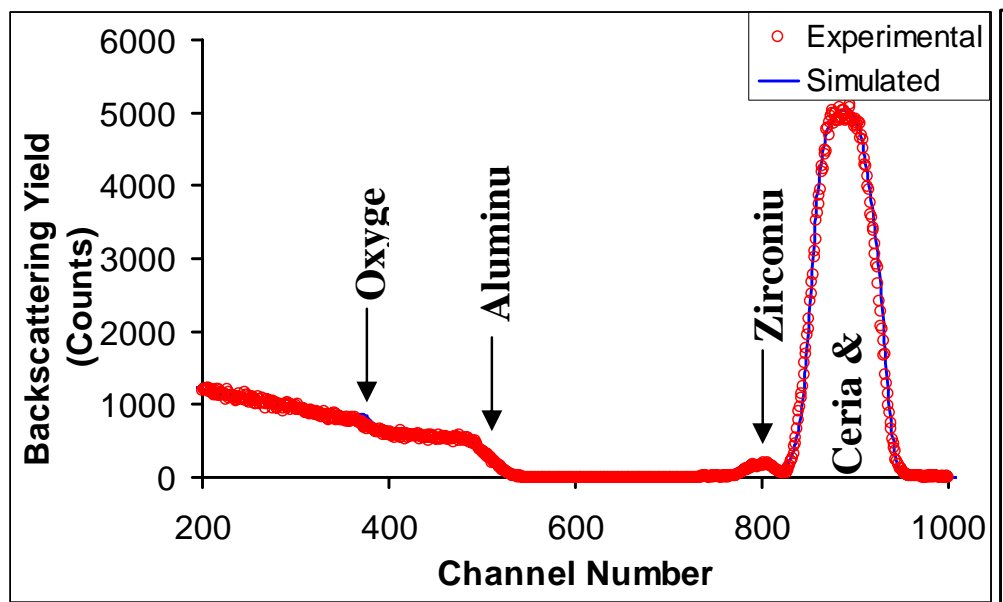

Fig 3: RBS spectrum of 12 at $\%$ Gd doped Ceria on Zirconia buffered Alumina layer

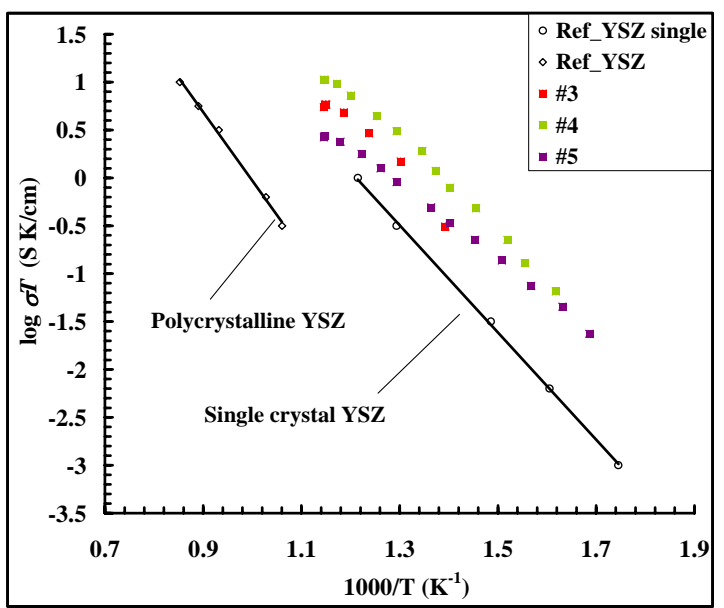

Fig 4: Arrhenius plots of oxygen ionic conductivity of various $\mathrm{Gd}_{2} \mathrm{O}_{3}$ doped ceria 


\title{
Time Resolved Laser Induced Fluorescence Spectroscopy (TRILFS) Exploration of Uranyl Adsorption on Gibbsite in $\mathrm{CO}_{2}$ free environment
}

\author{
Hyun-shik Chang and Gregory V. Korshin \\ Department of Civil Engineering \\ University of Washington \\ Seattle, WA \\ And \\ Zheming Wang \\ Pacific Northwest National Laboratory \\ Richland, WA
}

\section{Introduction}

Hyun-shik Chang acknowledges participation in PNNL's Interfacial and Condensed Phase Summer Research Institute. During the summer of 2004, several series of TRLIFS experiments with gibbsite samples containing adsorbed uranyl were carried out at PNNL. The goals of these experiments can be outlined as follows:

- To determine main features of the emission spectra of uranyl adsorbed on gibbsite.

- To quantify the effects of variations of $\mathrm{pH}$ and ionic strength on these spectra in $\mathrm{CO}_{2}$ free condition.

- Based on the global analysis of the relevant spectroscopic data, to determine the number of independent components in the emission of uranyl and obtain their "fingerprints".

- To establish, at least tentatively, relationships between the occurrence and properties of distinct components in the emission spectra of adsorbed uranyl species and, on the other hand, possible identities of adsorbed species whose presence is hypothesized by the currently existing adsorption models.

The above list of goals is quite ambitious while the amount of time available to carry out all needed experimental activities and to troubleshoot sample preparation, handling etc. during the summer of 2004 was a limitation. Nonetheless, some fairly intriguing results concerning the spectroscopic characteristic of adsorbed uranyl have been obtained. They will be discussed in the sections that follow. A description of procedures employed to prepare uranyl-containing samples for TRLIFS experiments is given in the follow as well.

\section{Experimental}

The uranyl dose for the adsorption on gibbsite was determined by the previous experimental results conducted by Dr. Gregory V. Korshin. According to his results, 
uranyl dose $200 \mu \mathrm{g} / \mathrm{kg}$ of suspension showed excellent adsorption behavior on the gibbsite in ambient environment. The same dose was applied to all of experiment conducted this summer. Three different ionic strengths were selected by Dr. Zheming Wang, 0.001, 0.01, and 0.4M, respectively. Those ionic strengths were achieved with $\mathrm{NaOCl}$. The applied $\mathrm{pH}$ range was from 4.0 to 9.0 with 0.5 of increment. To adjust the $\mathrm{pH}, \mathrm{NaOH}$ and $\mathrm{HOCl}$ were used.

The surface area per one liter of solution was always $3.5 \mathrm{~m}^{2}$. All the experiments carried out inside of the glove box with $\mathrm{N}_{2}$ purging to maintain $\mathrm{CO}_{2}$ free condition. To avoid the contact with ambient atmosphere when those samples were outside of glove box, all samples were tightly capped, and sealed with Para film ${ }^{\circledR}$.

Sample preparation Uranyl-containing samples were prepared for spectroscopic measurements using the following sequence of procedures. A desired amount $(21 \mathrm{~g}$ in the experiments described in this document) of gibbsite (Alkan SF4 lot \#00617111) was placed in $200 \mathrm{~mL} 0.01 \mathrm{M} \mathrm{NaOH}$ and shaken for 30 minutes on a shaker table. Following that, the suspension was centrifuged for 1 hour at 11,000 rpm. The supernatant was then decanted and the suspension was shaken for $30 \mathrm{~min}$. in $200 \mathrm{~mL} 0.01 \mathrm{NaClO}_{4}$ and centrifuged at 11,000 rpm for 30 minutes. The supernatant was again decanted. Washing with $0.01 \mathrm{M} \mathrm{NaClO}_{4}$ followed by centrifugation was repeated five more times.

The $\mathrm{pH}$ of the supernatant after these procedures was 8.55 . The remaining hydroxide was then neutralized to $\mathrm{pH} 7.1$ with $\mathrm{HClO}_{4}$. The resulting gibbsite suspension was finally centrifuged at $11,000 \mathrm{rpm}$, washed with $0.01 \mathrm{M} \mathrm{NaClO}$. The stock suspension of purified gibbsite (in $0.01 \mathrm{M} \mathrm{NaClO}_{4}$ ) contained $105 \mathrm{~g} / \mathrm{L}$ gibbsite. The $\mathrm{pH}$ of that solution was 7.1 .

As per Steve Smith, specific surface areas of the gibbsite were $3.5 \mathrm{~m}^{2} / \mathrm{g}$. In all cases, the surface area exposed to uranyl-containing solutions was $3.5 \mathrm{~m}^{2} / \mathrm{L}$. The $\mathrm{pH}$ was adjusted with $0.1 \mathrm{M} \mathrm{NaOH}$ or $0.1 \mathrm{M} \mathrm{HClO}_{4}$. Experiments were carried our in $\mathrm{CO}_{2}$ free conditions. $\mathrm{CO}_{2}$ free water was prepared by boiling it for 2 or 3 hours, following which the water was transferred (while still hot) to a gas chamber. All stock solutions utilized were prepared with $\mathrm{CO}_{2}$ free water.

The uranyl-containing samples were allowed to equilibrate for at least a week. The $\mathrm{pH}$ of the supernatant was continuously monitored and adjusted every day. At the end of exposure, the samples were centrifuged at 11,000 rpm for an hour, and aliquots of supernatant were taken for analyses for remaining uranyl. Desired amounts of the uranylcontaining solid phases (gibbsite) were transferred to quartz cuvettes for further experimentation using TRLIFS.

Cryogenic U(VI) Fluorescence measurements Fluorescence spectroscopic and lifetime measurements were performed in a Cryoindustries $\mathrm{RC}-152$ cryostat at near liquid Hetemperature $(5.0 \pm 0.5 \mathrm{~K})$. Individual uranyl-containing solid samples in quartz cuvettes were mounted in custom-built sealed copper sample holders fitted with sapphire optical windows. The sample cell holder was directly exposed to the vapor flow of liquid helium. The sample temperature was controlled by tuning both the liquid helium flow rate and the 
electric current applied to the internal heater of the cryostat through a Lakeshore 330 auto-tuning temperature controller.

The fluorescence emission spectra were obtained by excitation into the first electronic absorption band of the uranyl. The resulting fluorescence was collected at $90^{\circ}$ to the excitation beam by a 2 in diameter $f / 3$ fused silica lens and focused by a 2 in f/4 fused silica lens into the entrance slit of a $0.3 \mathrm{~m}$ focal length Acton SpectroPro 300i double monochromator spectrograph. An Omega 460LP cutoff filter with a cutoff wavelength of $460 \mathrm{~nm}$ was placed in front of entrance slit of the spectrograph to reject the scattered laser light.

Time-resolved emission spectra were recorded using a thermoelectrically cooled Princeton Instruments PIMAX time-gated intensified CCD camera that was triggered by the delayed output of the laser pulse and controlled by the WinSpec ${ }^{\mathrm{TM}}$ data acquisition software. The fluorescence lifetime of U(VI) of the samples was measured using a Hamamatsu R928 photomultiplier tube (PMT) after dispersing through a CVI R110 monochromator. The fluorescence intensity signal from the PMT was amplified and recorded with a Tektronics TDS 754A digital oscilloscope.

\section{A Short Description of Time-Resolved Emission Spectra}

Emission spectra of adsorbed uranyl were recorded in two basic modes. Integrated spectra were recorded using a $204.5 \mu$ s delay time and $1 \mathrm{~ms}$ gate time. Time resolved spectra were recorded for delay times varying from 204.5 to $1204.5 \mu$ s while the gate time was kept constant at $100 \mu \mathrm{s}$. Increase of the delay time was always accompanied by consistent, albeit sometimes subtle, changes of the shape of emission spectra. Many examples of this can be given but in most cases essentially the similar behavior was exhibited as shown in Figure 1.

Spectra represented in these figures demonstrate that the integrated emission for a $1 \mathrm{~ms}$ gate time comprised several spectroscopically distinct components. Their number and properties will be discussed in the next section. The increase of the delay time was accompanied by the appearance of distinct vibronic lines that were blue-shifted compared to the position of the component most prominent at short delay times (Figure 1).

The contributions of the long-lived components tended to increase with the $\mathrm{pH}$. However, this observation does not allow per se quantifying the "hidden" structure of the emission spectra of adsorbed uranyl and trends associated with varying conditions. The actual contributions and number of distinct emitting species of uranyl adsorbed on gibbsite were determined based on the global analysis of the time-resolved spectra obtained for varying delay times, pHs, and ionic strengths. The relevant results are present in the next section. 


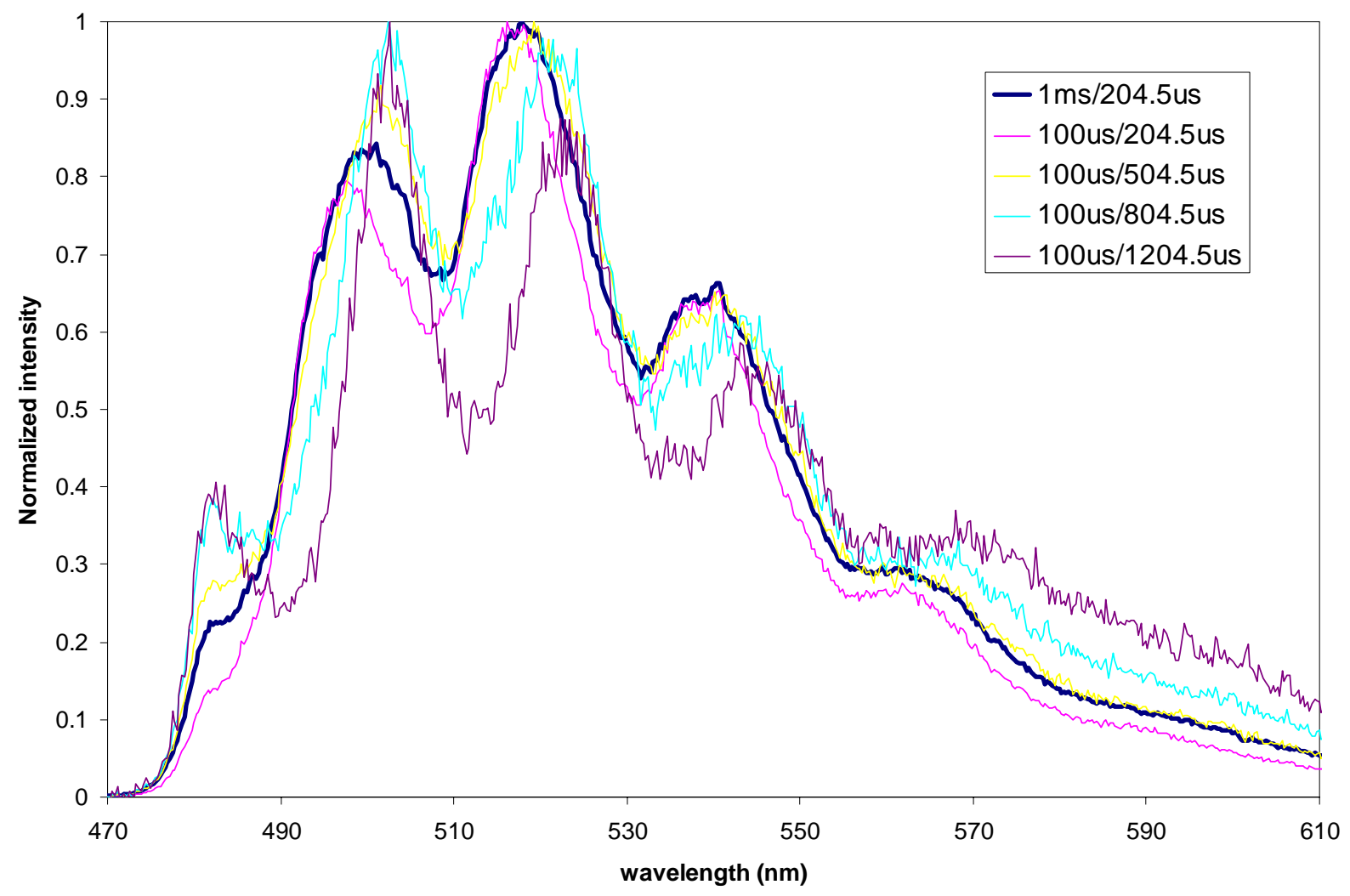

Figure 1 Series of time-resolved normalized emission spectra for uranyl adsorbed on gibbsite. Data for the uranyl dose $200 \mu \mathrm{g} / \mathrm{kg}, \mathrm{pH}$ 8.0, I.S. $0.001 \mathrm{M}$, and varying time delays

\section{Deconvolution of the Emission Spectra of Adsorbed Uranyl}

The goal of numerical deconvolutions and allied procedures was to use the time-resolved spectra for either of the substrates and achieve a global fitting that would adequately and hopefully unambiguously determine the number of components and their relative contributions.

To do that, evolving factor analysis (EFA), an applied mathematical technique, was used. The fundamentals of EFA can be outlined as follows. When the measured spectrum matrix is described as $\mathbf{X}(\mathrm{Mx} \mathrm{N})$, it can be decomposed into two matrixes such as $\mathbf{X}=$ C.S. The dimension of $\mathbf{C}$ is $\mathrm{M} \times \mathrm{K}$, and that of $\mathbf{S}$ is $\mathrm{K} \times \mathrm{N}$. $\mathrm{K}$ is the number of components, and it is determined by EFA. Using iterations combined with fit quality examinations by the least square method, the actual $\mathbf{C}$ and $\mathbf{S}$ matrixes can be calculated.

After then, the deconvoluted spectra (S) would be further processed using Gaussian peak analysis (GPA) if time allowed. Through these steps the spectroscopic characteristics of each "signature" spectrum could be examined to determine its characteristics (e.g., positions, width and relative intensities of each Gaussian-shaped line). 
The basic sequence of numerical data processing was to determine first the number of components in the spectra through EFA and, second, to separate the spectra using multivariate curve resolution (MCR). To do so, evovfa function for EFA and $\mathrm{mcr}$ function for MCR in PLS toolbox ${ }^{\circledR}$ of Matlab ${ }^{\circledR}$ were employed. The evolvfa function performs forward and reverse EFA, and calculates the singular values (square roots of the eigenvalues of the covariance matrix) of sub-matrices of input data $(\mathbf{X})$, and returns results of the forward analysis and reverse analysis. The $\mathrm{mcr}$ function executes iteration to find proper $\mathbf{C}$ and $\mathbf{S}$ matrixes using least square method.

EFA demonstrate the presence of three major components (Figure 2). All three components have 4 or 5 prominent vibronic lines. The properties of each vibronic line in these sequences and spacing between them can be discussed after finishing the GPA later. It can be mentioned here that components $\# 1$ and $\# 2$ obtained via EFA procedures are notably blue-shifted compared to component \#3.

\section{Conclusions and future study}

The results briefly presented above support the following conclusions:

1. EFA is efficient and promising approaches to process complex series of timeresolved emission uranyl spectra and attain a global fitting for data sets generated at varying $\mathrm{pH}$, ionic strength, and, potentially, other variable system characteristics.

2. For gibbsite, EFA indicates the presence of three major components operationally referred to as \#1, \#2, and \#3.

3. Components \#1 and \#2 are blue shifted compared to component \#3.

Those data and results shown above are only preliminary part of analysis to accomplish the goal of this research. Next step should include the effect of $\mathrm{pH}$ on contributions of spectroscopically distinct components, initial comparison of the emission spectra of adsorbed uranyl species and individual dissolved uranyl complexes, and so on.

I want to acknowledge, one more time, participation in PNNL's Interfacial and Condensed Phase Summer Research Institute, and thank Zheming Wang for his advices. 


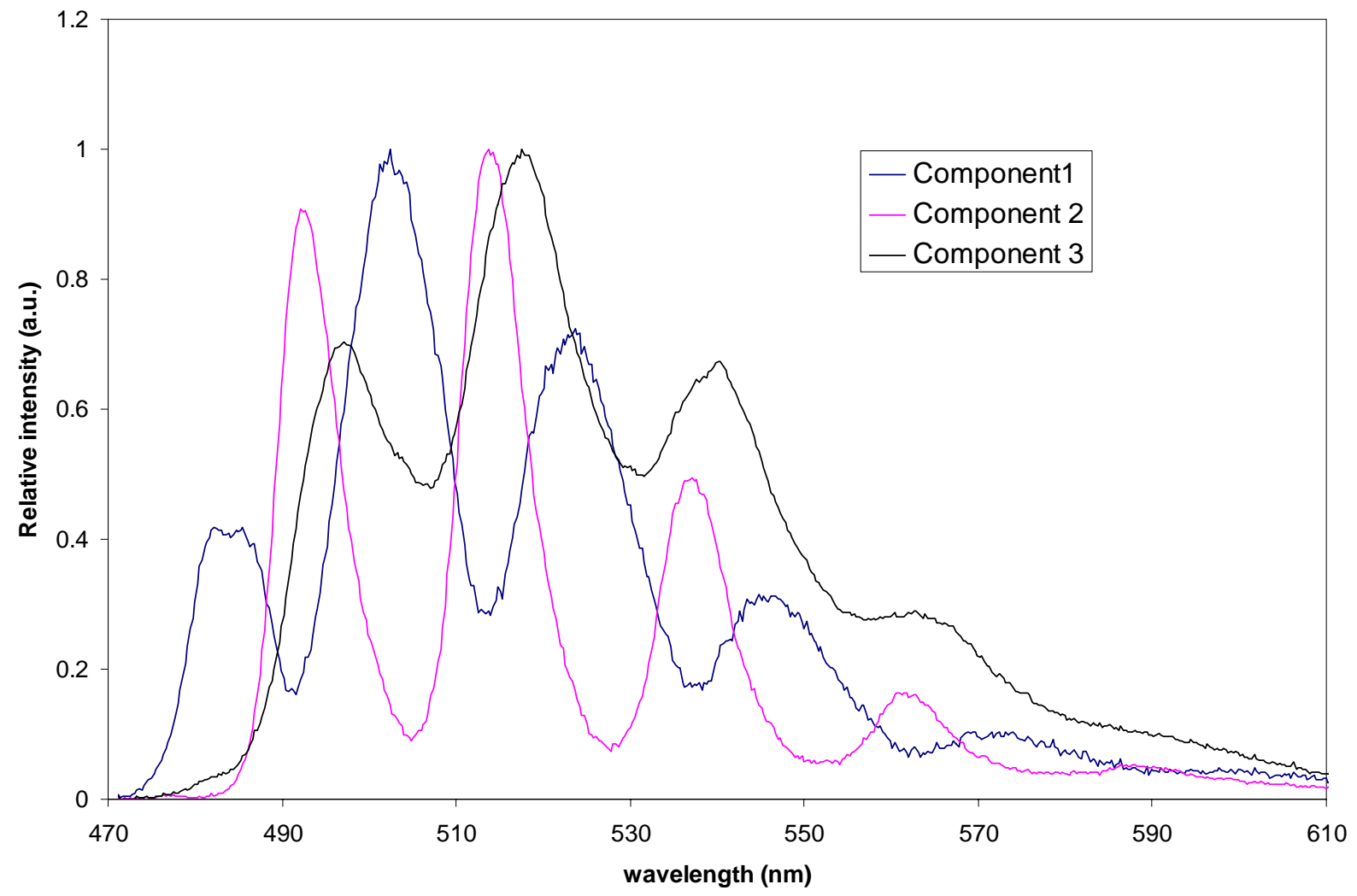

Figure 2 Normalized spectra of three major emitting uranyl species adsorbed on gibbsite. The data are obtained by deconvolution of series of uranyl emission data at varying ionic strengths, $\mathrm{pHs}$, and delay times. 


\title{
FIB Directed Self-assembly of Metal Oxide Nanostructures
}

\author{
Yingge Du and James F. Groves \\ Materials Science and Engineering Dept. \\ University of Virginia \\ Charlottesville, VA \\ and \\ Igor Lyubinetsky \\ Pacific Northwest National Laboratory \\ Richland, WA
}

\section{(a) The aim of the work and its relationship to the status of the field}

As the dimensions of electronic devices continue to scale down, traditional lithography is challenged to enable ever higher levels of feature integration. Focused ion beams (FIB) are being investigated as versatile maskless lithography tools that enable nano-scale feature definition. Our experiments were designed to explore FIB lithography techniques and their ability to guide the self-assembly of metal oxide islands on single crystal metal oxide substrates. We are interested in metal oxide systems because of their broad range of useful properties, including electrical functionality that covers the entire range from conductors to semiconductors and insulators. Additionally, many metal oxide materials are stable in open air environments. An FEI series 200 focused ion beam was used to implant localized dosages of $\mathrm{Ga}^{+}$into a $\mathrm{SrTiO}_{3}(100)$ substrate at a beam energy of $30 \mathrm{keV}$. The FIB implants generated an ordered array of surface topography, and these defined templates controlled quantum dot growth (Figs. 1 and 2). Our demonstrated ability to direct the self-assembly of $\mathrm{Cu}_{2} \mathrm{O}$ islands on $\mathrm{SrTiO}_{3}$ substrates has never been reported in metal oxides and could be extended to other metal oxide systems useful in a host of applications.

\section{(b) The work I have done during the Interfacial and Condensed Phase Chemical Physics Summer Research Institute}

Recent studies within the University of Virginia's Center for Nanoscopic Materials Design demonstrated guided self-assembly of Si-Ge quantum dots by FIB patterning. My advisor James Groves and I were stimulated by this idea and thought it might work for metal oxide systems exhibiting similar quantum dot formation phenomena. Through a collaborative research effort involving the University of Virginia (UVa) and Pacific Northwest National Laboratory (PNNL), I have demonstrated for the first time the synthesis of nanoscale arrays of cuprous oxide quantum dots on FIB patterned $\mathrm{SrTiO}_{3}(100)$ surfaces. I used the FIB system at UVa for initial patterning and the oxygen-plasma-assisted molecular beam epitaxy system at PNNL for cuprous oxide quantum dot synthesis. I worked with PNNL researchers on this project during the summer of 2003. Based on that initial success, I was funded by Battelle's Office of Fellowship Programs in the summer of 2004 to continue work on this project.

One of the most challenging parts of the work is associated with the FIB lithography. What implant dosages and spacings should I use? To address this problem, I first studied island distributions on non-patterned surfaces for an estimate of lithographic spacing. The ultimate metal oxide growth mechanism appears sensitive to both spacing and dosage of the local $\mathrm{Ga}^{+}$ implant. On each sample, I eventually made 15 different patterns. My studies have found that, to get one quantum dot for each FIB modified spot (and no dots in unmodified regions), the optimum spacing is related to the amount of material deposited, the size of the FIB-induced surface feature, and the diffusion length of $\mathrm{Cu}_{2} \mathrm{O}$ on $\mathrm{SrTiO}_{3}$, information not readily available in the literature. In the summer of 2003, I also observed another fascinating phenomenon, that for high dosage implant zones with relatively large spacing, it was possible to motivate the growth of 
multiple nanodots in repeatable locations around each implant. During 2004 I refined the FIB process, putting different implant dosages on different spots. Analyzing these samples required quite a bit of work. For example, on each sample I took atomic force microscopy (AFM) images of all 15 patterned regions after FIB modification, surface preparation and annealing, and after deposition. This took about 20 hours for each sample! Under one selected implant condition, oneto-one correspondence growth occurred as shown in Figure 2. Through a set of experiments, I was able to observe how material filled the holes first before islands formed.

\section{(c) Significant results of the work}

The research has demonstrated that the location of $\mathrm{Cu}_{2} \mathrm{O}$ quantum dot growth can be controlled in two ways. For high $\mathrm{Ga}^{+}$implantation $\left(\sim 4.4 \times 10^{8} \mathrm{Ga}^{+}\right.$per spot), the quantum dots can be grown at the edges of the implant zones (Fig. 1). For low implantation, $\left(\sim 6.3 \times 10^{3} \mathrm{Ga}^{+}\right.$per spot), almost all the dots have grown on top of the implant zones (Fig. 2). This basic research, studying and developing highly ordered nanoarrays of cuprous oxide quantum dots on $\mathrm{SrTiO}_{3}$ substrates, serves as a useful model system for study of guided self-assembly in metal oxides. This system and related metal oxide systems could hold the prospect for delivering hydrogen generation via direct water splitting (i.e., photocatalysis). More broadly, the techniques developed through my work should be applicable to other metal oxide systems. For instance, $\mathrm{NbO}_{2}$ islands on $\mathrm{TiO}_{2}$ have the potential for use as the foundation of bionano scaffolds with commercial potential in the areas of medicine, the environment, and security. Epitaxial growth of $\{100\}$ faceted ferromagnetic $\mathrm{CoCr}_{2} \mathrm{O}_{4}$ clusters on $\mathrm{MgAlO}_{4}(001)$ surface has been reported recently in the literature. If we can arrange such clusters in a defined way, they could be used as high capacity memory storage. Additionally, the ability to define metal oxide nanodot growth location has broad implications for incorporating such nanostructures into next generation nanoelectronic and spintronic devices. In sum, the FIB lithography technique demonstrated by my research opens up a new realm of materials opportunities - guided self-assembly of metal oxides. As other researchers apply my technique to other metal oxide systems, even more useful applications could be realized.

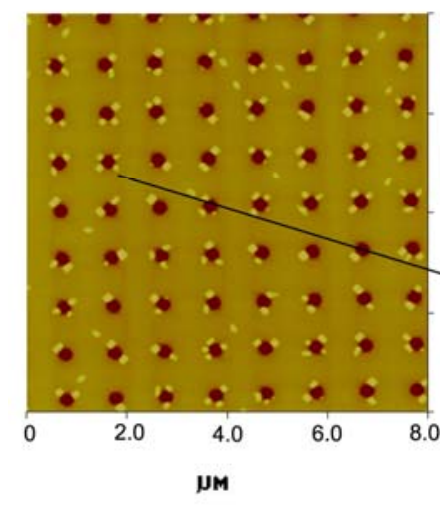

(a)

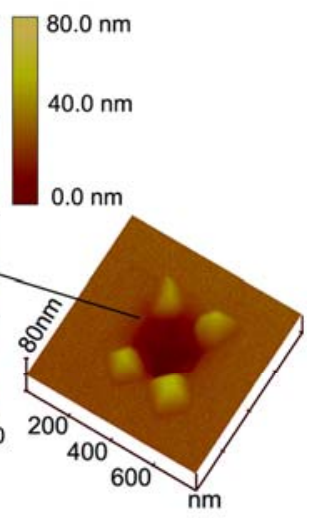

(b)

FIG. 1. (a) AFM image showing preferential growth of $\mathrm{Cu}_{2} \mathrm{O}$ quantum dots on edges of FIB implant zones, (b) a high resolution scan of one FIB implant zone. $\mathrm{Cu}_{2} \mathrm{O}$ islands are light spots while the FIB implant zones are dark spots. 


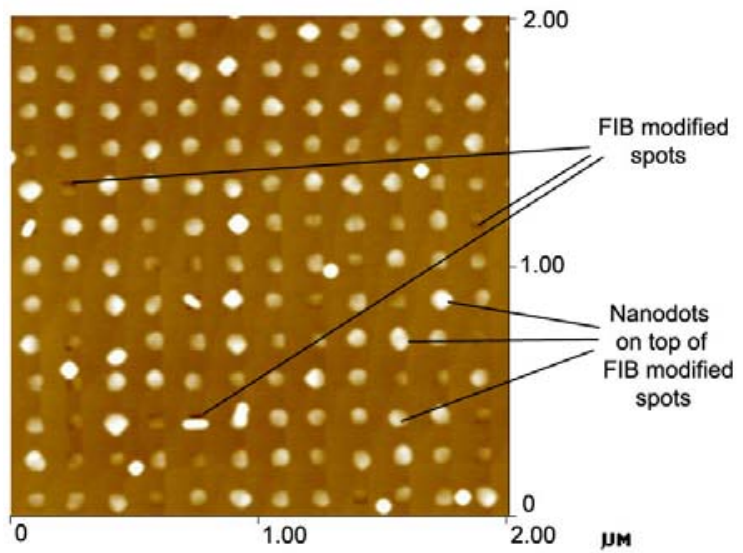

FIG. 2. AFM image showing the preferential growth of $\mathrm{Cu}_{2} \mathrm{O}$ quantum dots on the top of FIB implant zones. The quantum dots are light spots on the $\mathrm{SrTiO}_{3}$ single crystal surface. Quantum dot growth location has been defined through FIB pre-patterning of the growth surface. defined through FIB pre-patterning of the growth surface.

\section{Publication or presentation generated:}

MRS Fall Meeting at Boston

CONTACT ( NAME ONLY): Yingge Du

Abstract Details

PRESENTATI ON TYPE: Oral Presentation Preferred

SYMPOSI UM: C: Innovations for Sub-100nm Lithography-Materials and Processes

KEYWORDS: Focused ion beam, templates, maskless lithography.

\section{Abstract}

TITLE:

Fabrication of Ordered Quantum Dot Arrays by Focused I on Beam Lithography

AUTHORS (ALL): Du, Yingge ${ }^{1}$; Groves, James $\mathrm{F}^{1}$; Lyubinetsky, Igor ${ }^{2}$; Thevuthasan, Suntharampillai ${ }^{2}$.

I NSTI TUTI ONS (ALL): 1. University of Virginia, Charlottesville, VA, USA.

2. Pacific Northwest National Laboratory, Richland, WA, USA.

\section{ABSTRACT BODY:}

As the dimensions of electronic devices continue to scale down, traditional lithography is challenged to enable ever higher levels of nanoscale feature integration. In recent years focused ion beams have begun to be used as versatile maskless lithography tools that enable nano-scale feature definition. In this work, an FEI series 200 focused ion beam was used to implant localized dosages of $\mathrm{Ga}^{+}$into a $\mathrm{SrTiO}_{3}(100)$ substrate at a beam energy of $30 \mathrm{keV}$. The FIB implants generated surface topography with lateral dimensions of 30-70 $\mathrm{nm}$ and implant spacings of 83 $\mathrm{nm}$ to $170 \mathrm{~nm}$. Carefully controlled ion dosages were implanted into different regions of the substrate, and the morphology changes were characterized by atomic force microscopy. During subsequent substrate annealing, the implanted spots evolved to well-defined, square-based holes because of recrystallization.

Following substrate patterning, annealing, and cleaning, the FIB template served as the guide for fabrication of ordered quantum dot arrays. Oxygen plasma assisted molecular beam epitaxy was used to grow $\mathrm{Cu}_{2} \mathrm{O}$ quantum dots on the FIB patterned 
$\mathrm{SrTiO}_{3}$ substrates. In-situ X-ray photoelectron spectroscopy and Auger electron spectroscopy analysis demonstrated that the final phase of the nanodots was $\mathrm{Cu}_{2} \mathrm{O}$. Under selected conditions, the nanodots preferentially nucleate inside the holes and then grow in size on top of the FIB induced topography. Observation reveals that one quantum dot grows on each FIB nanoscale topographic feature. Initial analysis has been performed on the fabricated samples to quantify quantum dot size, size distribution and inter-dot spacing, important characteristics for next generation nanoelectronic applications. 


\title{
Computational Investigation of the structures of the $\mathrm{Fe}_{2} \mathrm{~S}_{2}{ }^{-}$anion
}

\author{
Benjamin M. Elliot and Alexander Boldyrev \\ Chemistry Department \\ Utah State University \\ Logan, UT \\ And \\ Lai-Sheng Wang \\ Physic Department \\ Washington State University, Tri-Cities \\ Richland, WA
}

The project was based upon the work done in the fellowship during the summer of 2003. The premise was the collaboration of experiment and theory in the elucidation of the electronic structure of iron-sulfur clusters. Photoelectron spectra were run on the species of interest by the group of Lai-Sheng Wang at EMSL. The structure under question was the $\mathrm{Fe}_{2} \mathrm{~S}_{2}{ }^{-}$anion in various forms.

The lowest energy states were elucidated using the ADF calculation program run on the mpp2 super computer in EMSL. The experimental spectra were compared to calculated spectra which were also run at EMSL. Using the "fingerprint" spectra the lowest energy states could be identified and shown to be the experimental result as well. The ground states of the $\mathrm{Fe}_{2} \mathrm{~S}_{2}{ }^{-}$were found. The calculations are almost finished, and will soon be completed for final consolidation of data to be set for publication. 


\title{
Holding single molecules in solution using magnetic tweezers: a new approach to studying DNA damage recognition by proteins.
}

\author{
Jason J. Han and Alex Li \\ Chemistry Department \\ Washington State University \\ Pullman, WA \\ And \\ H Peter Lu \\ Pacific Northwest National Laboratory \\ Richland, WA
}

\section{Research summary}

The research objective for the 10-week summer internship at PNNL was to demonstrate the technical feasibility of coupling single molecule spectroscopy (SMS) with magnetic tweezers as a new method for studying DNA-protein interaction on single DNA molecules in solution. This new method would combine SMS, which would provide dynamic and conformational information, with magnetic tweezers, which would be used to exert various forces (e.g.- tension, torsion) on a single DNA molecule, or to hold the DNA molecule in a specified orientation in solution space. As such, three distinct goals were identified as a major focus of the 10-week internship: 1) modify existing methodologies from the literature to prepare a sample suitable for SMS; 2) use magnetic fields to demonstrate the "magnetic tweezers" effect; 3) perform fluorescence spectroscopy on a single molecule of stained DNA under physical control by magnetic tweezers. Goals 1 and 2 were achieved; however, a few experimental parameters remain in need of optimization. Goal 3 was attempted but not attained due to time constraints and experimental procedures that led to levels of background fluorescence unacceptable for single molecule experiments.

\section{Background}

Single molecule fluorescence spectroscopy is a technique that probes a single molecule with laser excitation and examines the molecule's resulting emission properties, which serve as a "reporter" of various types of information. The technique has been used extensively in recent years to gain insight into, amongst other areas, single biomolecular conformational dynamics. Numerous peer-reviewed articles on SMS can be found via common scientific search engines, where applications ranging from studies on photon-antibunching and electronic structure of organic dyes and quantum dots, to surface structure of thin films, to biomolecular conformation and dynamics can be seen. While no attempt is being made here to detail the field of SMS, it is important to note that single-molecule DNA-protein and protein-protein interaction dynamics studies have been demonstrated by Peter Lu at Pacific Northwest National Lab $(\mathrm{PNNL})^{1,2}$. 
In the well-developed magnetic tweezers technique for DNA micromanipulation ${ }^{3-}$ ${ }^{5}$, one end of a linear, end-functionalized DNA molecule is linked to a superparamagnetic microsphere, most commonly through the well-known biotin-streptavidin interaction, while the other end is tethered to a glass cover slip or capillary tube. Magnetic fields are then used to manipulate the magnetic particle so as to partially immobilize, stretch, compress, twist, or otherwise distort the DNA molecule (Figure 1) while exposing the DNA to proteins or enzymes in solution.

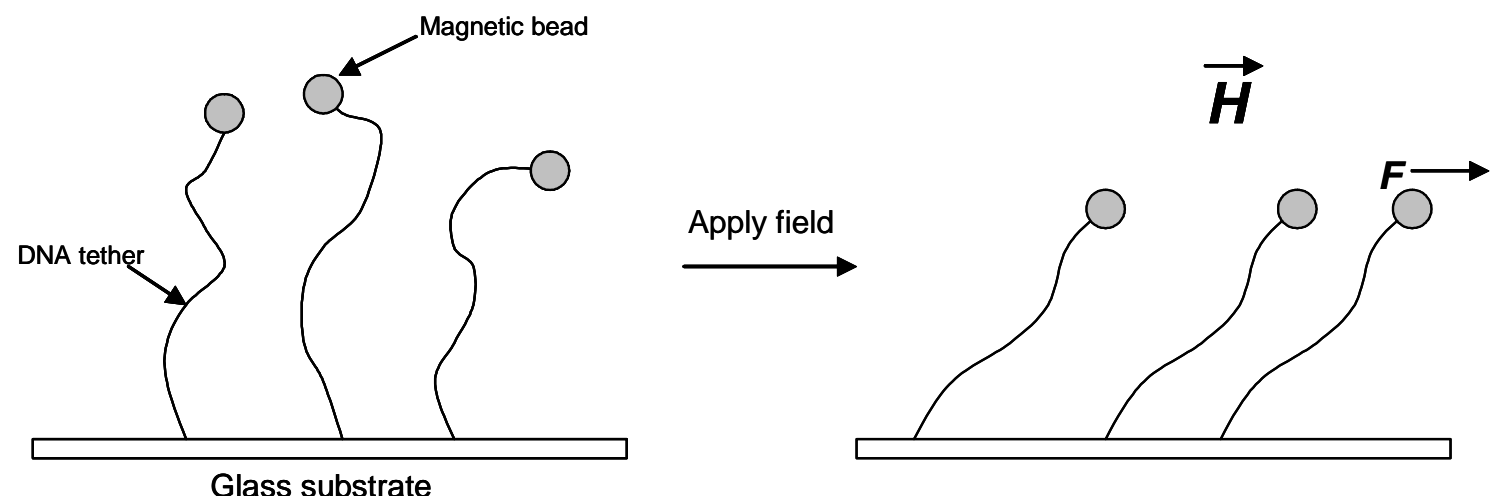

Figure 1. Illustration of magnetic tweezers effect in solution.

Such micromanipulation has been dubbed "magnetic tweezers." A number of magnetic tweezers studies have focused on quantifying forces responsible for holding DNA together in its native state, while others have focused on how these forces change as a result of interaction with another biomolecule such as a protein or enzyme.

Both SMS and magnetic tweezers techniques are well-developed to date. Yet, although their simultaneous integration has been suggested ${ }^{6}$, such realization remains unreported in the literature. The scientific interest in combining the two methods lies in the ability to impose varying degrees of physical control over a molecule's motion in solution while subjecting the molecule to chemical perturbations. Physical control can include, for example, applying tension or torsion to a linear, or linearizable, polymer or biomolecule by applying a magnetic field that causes the magnetic particle to move in a way that stretches, or linearizes, the molecule. Chemical perturbations can include, for example, exposing the subject molecule to an interacting chemical species, such as an enzyme, or changing the solvent environment to change the solubility properties of the subject molecule in solution. By combining SMS with magnetic tweezers, one will be able to probe the fluorescence properties (e.g. - FRET) of a molecule while imposing a level of physical motion control that has yet to be reported in the SMS field. Such control will open new doors to the study of single molecule interactions and folding. 


\section{Methods and materials}

\section{General overview.}

The procedure for tethering magnetic beads to a glass cover slip was adapted from published methods $s^{4,7,8}$. This modified procedure utilizes the cohesive ends of linearized $\lambda$-DNA and the well-known biotin-streptavidin interaction to tether a superparamagnetic micron-sized bead to a glass cover slip, where $\lambda$-DNA serves as the tether.

Lambda DNA is a $48 \mathrm{~kb}(48,502$ base pairs) duplex DNA that can be partially denatured with heat from its circular form to yield the linear form, which has a contour length of $\sim 17 \mu \mathrm{m}$ and contains cohesive ends (Figure 2). Each cohesive end is $12 \mathrm{bp}$ (base pairs) long, and can be chemically modified by a variety of methods.

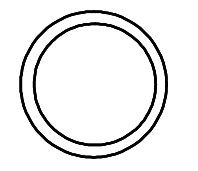

Circular $\lambda$-DNA
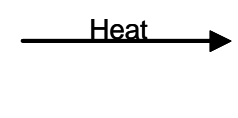

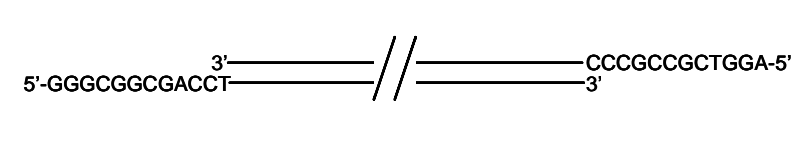

Linear $\lambda$-DNA

Figure 2. Denaturation of circular $\lambda$-DNA to linear $\lambda$-DNA showing resulting 12 bp long cohesive ends, called 3 ' overhangs.

In this procedure, custom-synthesized oligo(nucleotides) complementary to the cohesive ends were purchased and used to chemically modify each end of $\lambda$-DNA. One end was modified with thiol, the other with biotin. The thiol moiety was used to chemically link via disulfide formation to mercaptosilane, which was subsequently used to tether the modified $\lambda$-DNA to a glass cover slip via siloxane bonds. The free biotinmodified end of $\lambda$-DNA was then exposed to streptavidin-coated superparamagnetic microbeads, resulting in attachment of the bead to the DNA tether by the strong biotinstreptavidin interaction. Magnetic fields were imposed on the final construct using both a permanent magnet and a custom built magnetic tweezers apparatus. After confirming that beads were indeed tethered, the DNA sample was stained and subsequently inspected under laser excitation.

\section{Materials.}

Phage $\lambda$-DNA and T4 DNA ligase were purchased from New England Biolabs and used as received or diluted prior to use. Mercaptosilane (3mercaptopropyltrimethoxysilane), dithiothreitol (DTT), and Tween 20 were purchased from Aldrich and used without further purification. POPO-3-iodide was purchased from Molecular Probes and diluted in water prior to use.

Streptavidin-coated magnetic beads $\sim 1.5 \mu \mathrm{m}$ in diameter were purchased as a kit from Biomag (Biomag Plus Streptavidin Particle Biotin Binding Starter Kit). Bead purification was performed by washing $0.5 \mathrm{~mL}$ of bead concentrate three times in Coupling Buffer per instructions, then diluting to $6 \mathrm{~mL}$ (12X dilution) with Coupling Buffer, and finally adding $6 \mu \mathrm{L}$ of Tween 20 plus $300 \mu \mathrm{L} 1 \mathrm{M} \mathrm{NaCl}$. Suspensions were checked for undesirable aggregation under a light microscope. If necessary, suspensions were sonicated for a few minutes and rechecked under the microscope. 
Sodium acetate (NaOAc, $30 \mathrm{mM}, \mathrm{pH}$ 4.3), TE (1X, $10 \mathrm{mM}$ Tris-HCl, $1 \mathrm{mM}$ EDTA, $\mathrm{pH}$ 8.0), and TETw (TE with $0.1 \%$ Tween 20 ) buffers were freshly prepared from chemicals purchased from Aldrich and were stored at $4^{\circ} \mathrm{C}$

Glass cover slips (Gold Seal) were purchased from Fisher and soaked in 10\% $\mathrm{NaOH}$ for $>30$ minutes, rinsed thoroughly in DI water, then Millipore water, and dried at $>100^{\circ} \mathrm{C}$ for at least 2 hours prior to use.

\section{Oligonucleotides.}

Two batches of single stranded oligos were purchased from W.M. Keck Facility at Yale University and supplied as $200 \mathrm{nmole}$ dried form. The sequences of each oligo are complementary to the cohesive ends of $\lambda$-DNA. The 3 ' end is terminated with either 3' Biotin-TEG or 3'-Thiol-Modifier C3 S-S modifiers. Each oligo is terminated by a phosphate at the 5 ' end for ligation.

1) Biotin-terminated oligo sequence: 5'phosphate-GGG-CGG-CGA-CCT-3'biotinTEG

Structure of biotin modifier:

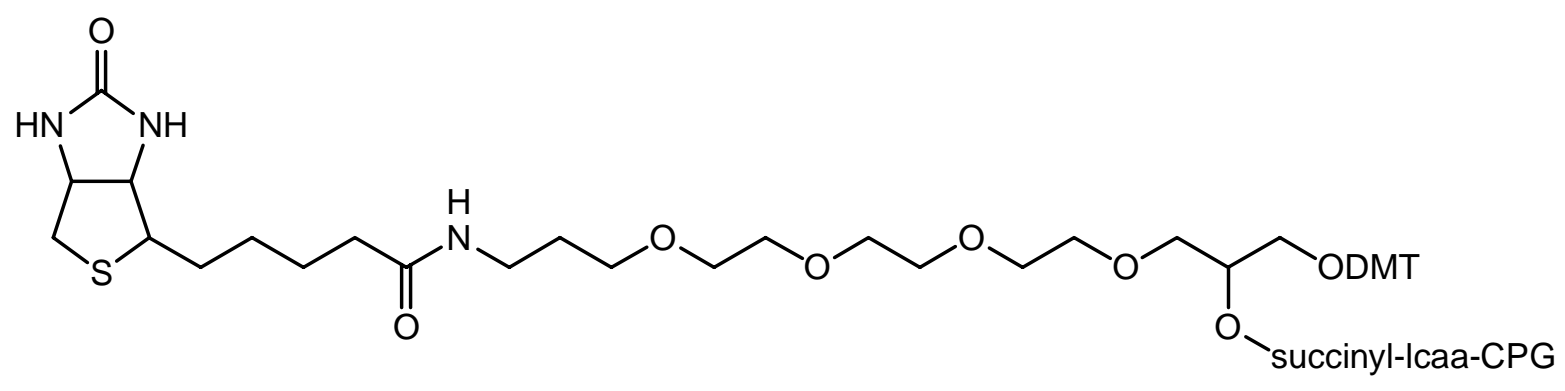

1-Dimethoxytrityloxy-3-O-(N-biotinyl-3-aminopropyl)-triethyleneglycolyl-glyceryl2-O-succinyl-long chain alkylamino-CPG

2) Disulfide-terminated oligo sequence: 5'phosphate-AGG-TCG-CCG-CCC-3'-S-SC3

Structure of 3'-Thiol-Modifier C3 S-S modifier:

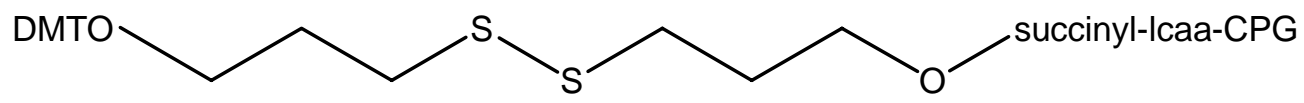

1-O-Dimethoxytrityl-propyl-disulfide,1'-succinyl-Icaa-CPG 
Each oligo batch was reconstituted by adding $1 \mathrm{~mL}$ TE buffer. Insoluble salts were removed by centrifugation. The resulting stock concentration for each oligo was $200 \mu \mathrm{M}$ (200 pmol/ $\mu \mathrm{L}$ ). A small aliquot of biotin-terminated oligo was further diluted with TE buffer to a working concentration of $0.1 \mu \mathrm{M}(0.1 \mathrm{pmol} / \mu \mathrm{L})$. The disulfide (thiol) oligo was used undiluted as the $200 \mu \mathrm{M}$ stock concentration.

\section{Sample preparation}

Sample preparation consisted of 5 major parts:

1) Deprotection and salinization of thiol oligo

2) Conjugation of salinized oligo to cover slip

3) Biotinylation of $\lambda$-DNA

4) Coupling of biotinylated $\lambda$-DNA to modified cover slip

5) Attachment of magnetic beads to tethered $\lambda$-DNA

1. Deprotection and silanization of disulfide-terminated oligonucleotide. Disulfide oligo was first deprotected (reduced) with dithiothreitol (DTT) to yield the thiol oligo. After purification with size-exclusion chromatography, the thiol oligo was coupled to mercaptosilane via disulfide formation to form the disulfidepropyltrimethoxysilaneterminated oligo, which was ready to conjugate to a glass surface (Scheme 1).

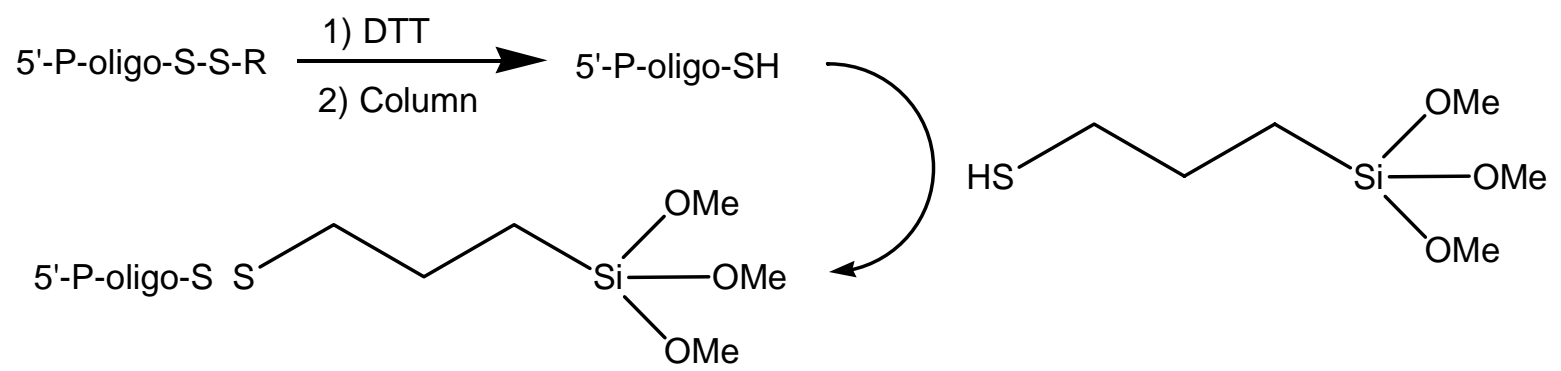

Scheme 1. Deprotection and salinization of thiol oligo.

In a microcentrifuge tube, $30 \mu \mathrm{L}$ disulfide oligo stock solution ( 6 nmoles) was mixed with $10 \mu \mathrm{L} 100 \mathrm{mM}$ aqueous DTT and reacted for $>30$ minutes at RT. The purified deprotected oligo was obtained by removing DTT on a homemade pre-equilibrated Sephadex G-25 column using NaOAc buffer as eluent. At least four, but no more than six $200 \mu \mathrm{L}$ fractions were collected and quickly analyzed on a UV-vis spectrometer to identify location of oligo as indicated by absorption at $260 \mathrm{~nm}$. After estimating mole quantities of oligo in each fraction (typically there were only two containing oligo), 10 equiv. of diluted mercaptosilane ( $5 \mathrm{mM}$ in $\mathrm{NaOAc}$ ) was added and the solutions were allowed to react for 2 hours at RT. After this period, the unpurified solutions were either used immediately for the next step, or stored in a $-20^{\circ} \mathrm{C}$ freezer for later use. This step yielded enough unpurified silanized oligo to treat many cover slips, so it did not need to be performed often. 
2. Conjugation of silanized oligo to glass cover slip. Conjugation was performed by "spotting" the unpurified silane/oligo reaction solution on the dry cover slip, and letting react at RT for $\sim 1$ hour in a covered Petri dish (Scheme 2).

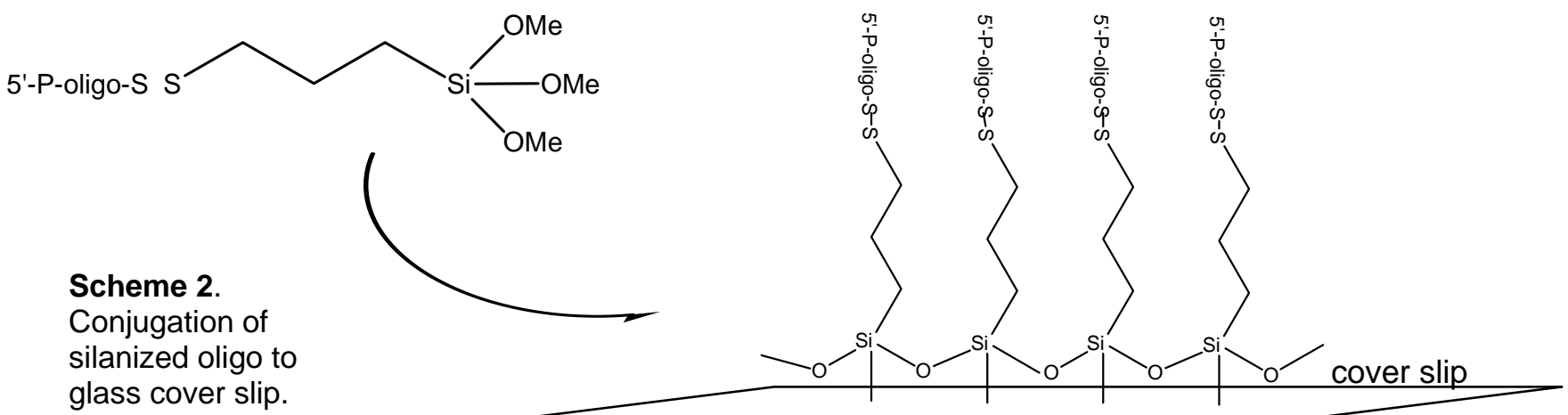

The reaction solution was spotted as both an array of small spots $(\sim 5 \mu \mathrm{L} / \mathrm{spot})$ and as
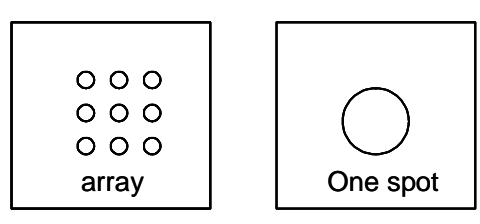
one large drop $(50-100 \mu \mathrm{L})$ in the center of the cover slip as shown in drawing. In the latter case, a circle was drawn in the center of the cover slip with a fine point permanent marker to aid in keeping track of the treated area of the cover slip in addition to serving as a hydrophobic barrier to help keep the reaction solution confined to within the circle.

After reacting, the Petri dish cover was removed and the reaction solution was allowed to evaporate at RT to dryness, using a gentle stream of $\mathrm{N}_{2}$ to facilitate complete evaporation. Salt deposits from the buffer were dissolved away by very gently rinsing the cover slips with Millipore water, after which the samples were placed in a $50^{\circ} \mathrm{C}$ oven for 5-10 minutes to dry and cure. Modified cover slips were either used immediately for the next step, or stored in Millipore water or TE buffer at $4^{\circ} \mathrm{C}$ for later use.

3. Biotinylation of $\lambda$-DNA. In a microcentrifuge tube, $50 \mu \mathrm{L}$ diluted $\lambda$-DNA $(10 \mu \mathrm{g} / \mathrm{mL}$ in TE buffer) was mixed with $3.3 \mu \mathrm{L}$ biotin oligo $\left(0.1 \mu \mathrm{M}\right.$ in TE buffer) and heated in a $65^{\circ} \mathrm{C}$ bath for 15-30 minutes to denature the cohesive ends. The oligos were annealed to cohesive ends by slowly cooling to RT and reacting for another 1 hour after reaching RT. After this period, $6 \mu \mathrm{L} 10 \mathrm{X}$ ligase buffer plus $1.5 \mu \mathrm{L}$ T4 DNA ligase were added. The solution was mixed with a pipet and reacted at RT for 2-4 hours. Higher concentrations of ligase required less reaction time (Scheme 3). 


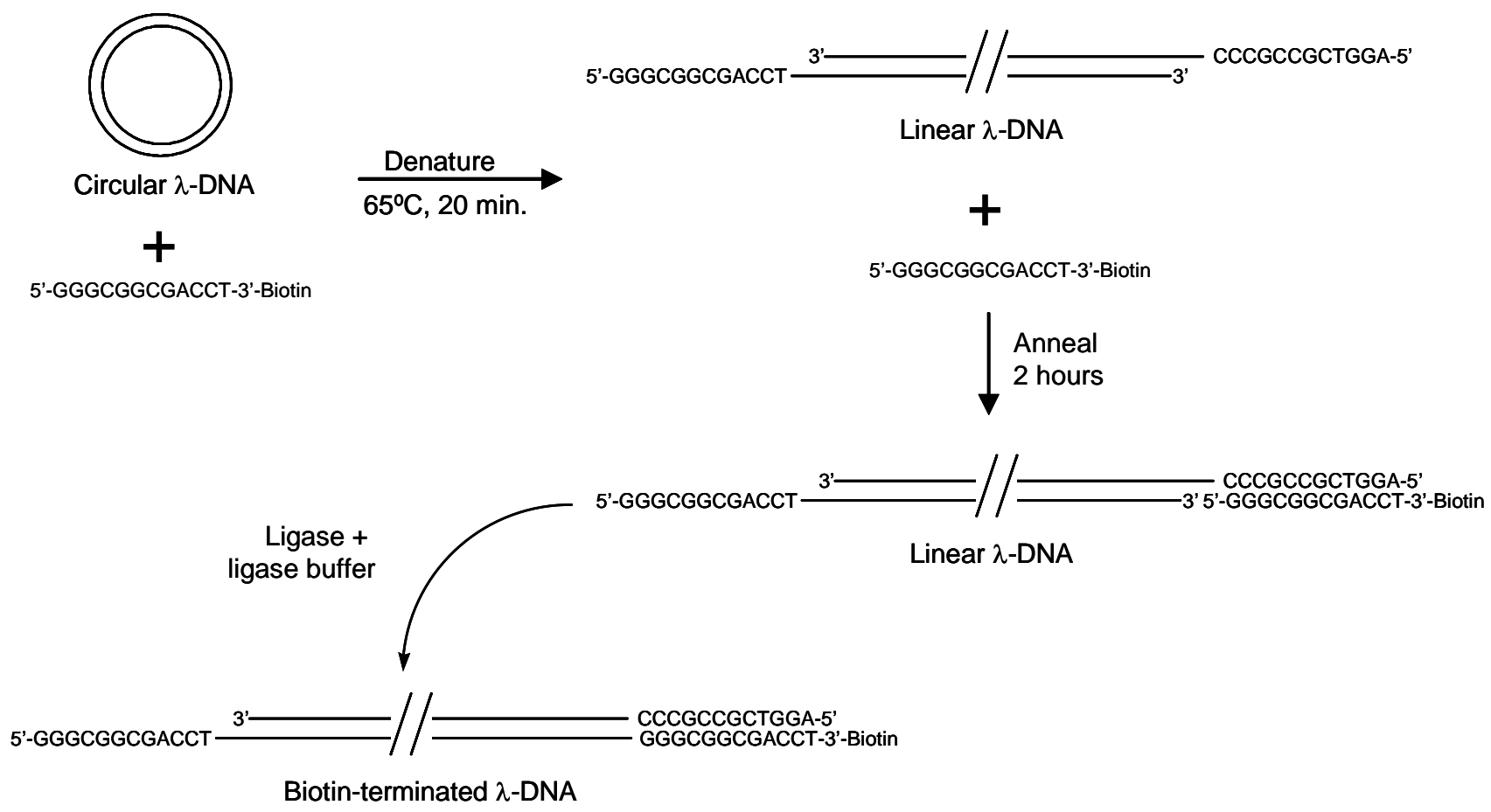

Scheme 3. Biotinylation of $\lambda$-DNA

4. Coupling of biotinylated $\lambda$-DNA to oligo-modified cover slip. After the biotinylation reaction period, enough of the reaction mixture was spread onto the cover slip so that the oligo-modified areas were covered. After 4-5 hours reaction time in a covered Petri dish, the samples were rinsed very gently with TETw buffer and used immediately in the next step or stored in TETw buffer at $4^{\circ} \mathrm{C}$ for no longer than two days until ready for next step (Scheme 4). 


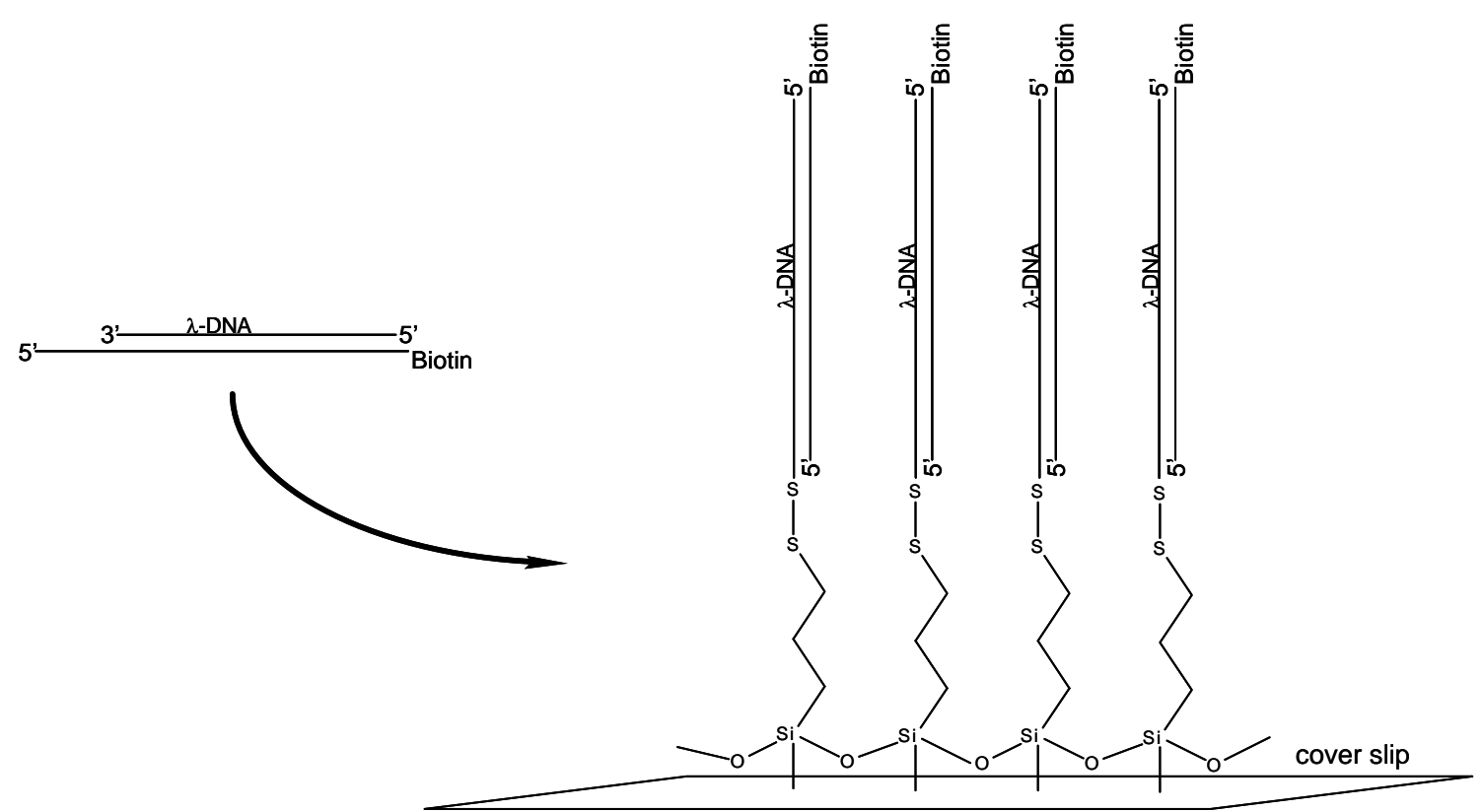

Scheme 4. Coupling of biotinylated $\lambda$-DNA to oligo-modified cover slip.

5. Attachment of magnetic beads. Bead suspensions were checked for aggregation on a microscope before using. After removing buffer from the modified area of the cover slip, enough (typically 50-100 $\mu \mathrm{L}$ ) of the diluted bead suspension was transferred so as to cover the modified area containing tethered biotinlyated $\lambda$-DNA. After 3-5 minutes, the unbound beads were gently rinsed away with TETw buffer, and the "active" area was replenished by pipetting enough buffer to form a stable liquid bead that covered the modified area. If not enough tethered beads were present under microscope inspection, the cover slip was retreated with magnetic beads in the same manner described. 


\section{Magnetic tweezers apparatus}

Two magnetic tweezers devices were custom built in the Instrument Development Laboratory (IDL) located in the Environmental Molecular Sciences Laboratory (EMSL) facility of PNNL. The first generation device is a two-pole device constructed from two small coils and sharpened metal rods as probes (Figure 3A). The coils used are rated as being able to withstand a constant drive voltage of up to $20 \mathrm{~V}$, though higher voltages can be, and were, used for short periods of time. The second generation magnetic tweezers device is similar to the first generation, except it contains four poles and larger coils, allowing it to be driven continuously at a minimum of $50 \mathrm{~V}$ (Figure 3B).
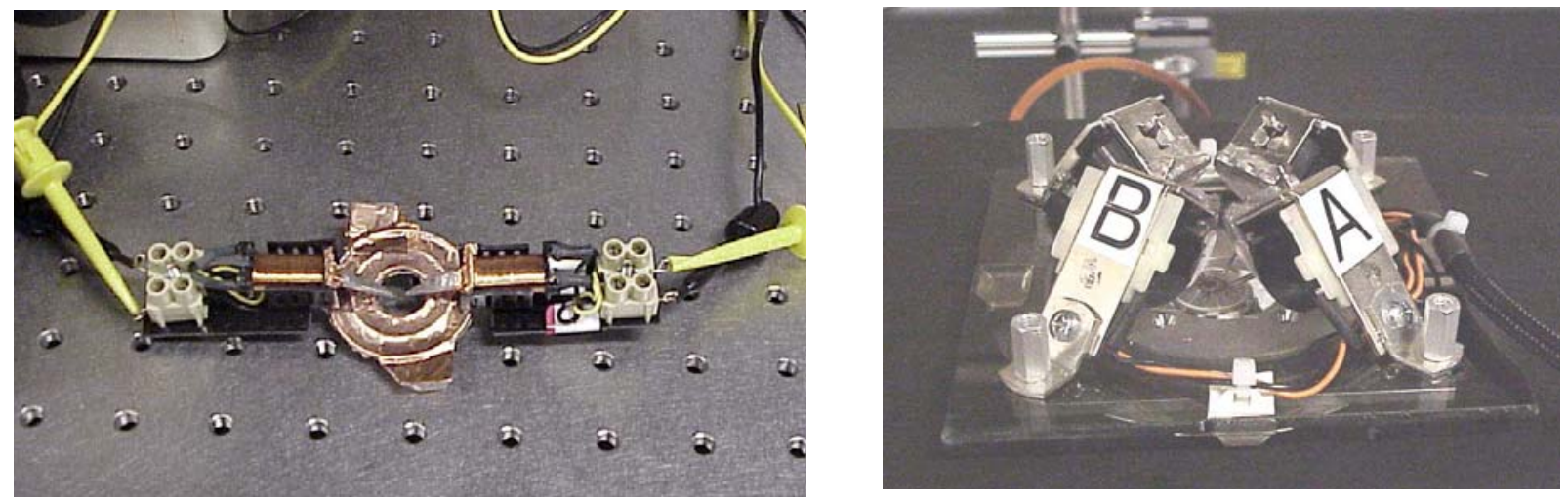

Figure 3. Two pole (A) and four pole (B) magnetic tweezers devices.

The devices were placed directly on top of the sample cover slip, which was resting on the translation stage of the microscope. Flux density and direction produced by the probes were not characterized prior to use on the sample.

Samples were also subjected to magnetic fields using a small but strong permanent magnet attached to an aluminum block for easy and stable manual manipulation around the translation stage. Because the voltage driven apparatus was not well characterized, this permanent magnet proved the easiest and most consistent and reproducible means to obtaining a well-defined response from the tethered beads. Figure 4, while grossly out of scale, shows a diagram depicting the relative orientation of the permanent magnet to the sample. As shown, the magnet is angled above the sample, and hence beads are drawn towards the magnet in an upwards diagonal trajectory. 


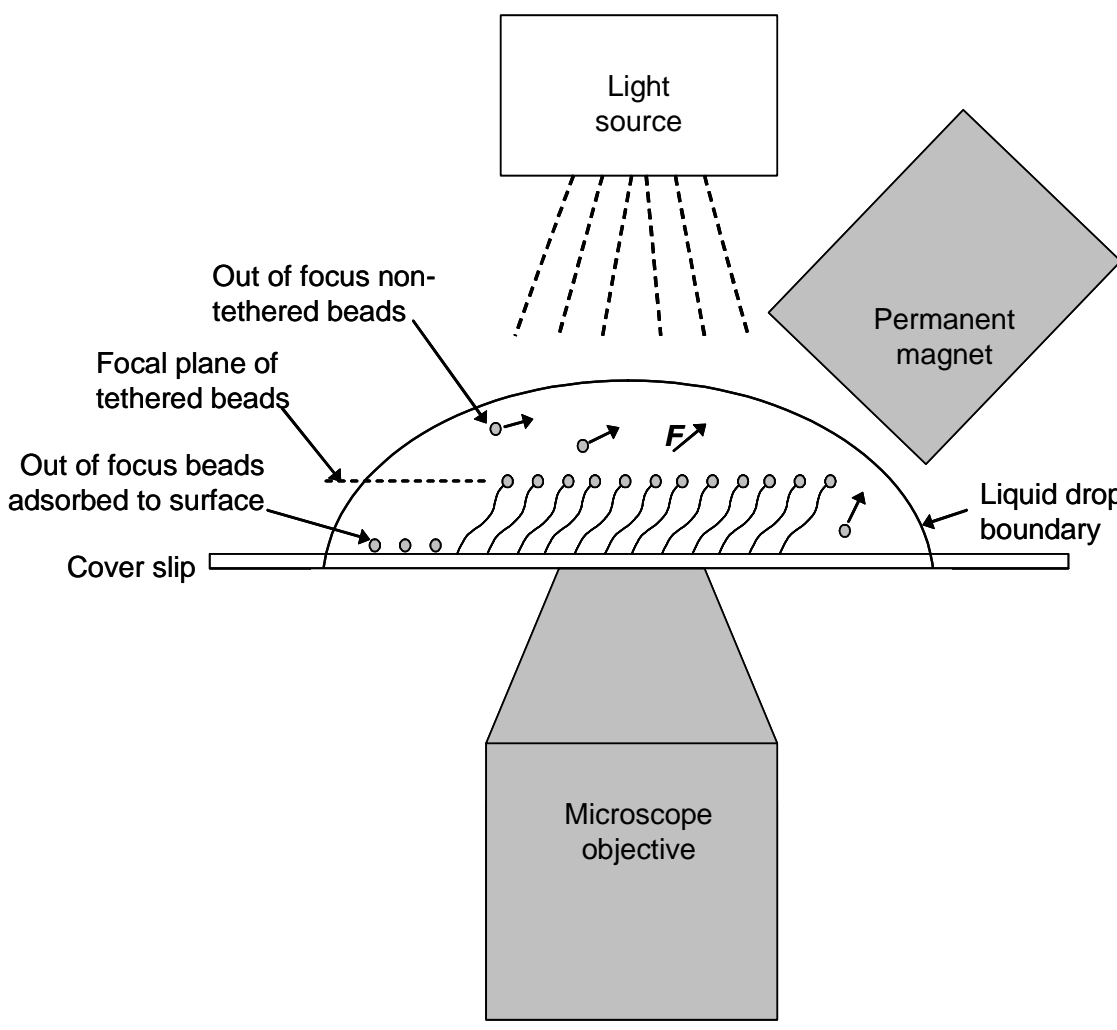

Figure 4. Illustration showing both tethered and non-tethered beads responding to a permanent magnet. Note that "free floating" beads may float into and out of the focal plane as they migrate towards the magnet (or diffuse in the absence of a magnet). The relative angle of the permanent magnet to the sample drop was approximately as depicted. Beads non-specifically adsorbed to glass surface often served as points of aggregation if strong enough fields were imposed.

\section{Optical and fluorescence microscopy}

Samples were inspected by light microscopy on a Nikon DIAPHOT 300 inverted microscope equipped with a 60X, 1.3 NA oil immersion lens. Color digital videos of tethered beads responding to magnetic fields were recorded with a World Precision Instruments eyepiece camera model DEC-18, which allowed easy capture at an acceptable frame capture rate simply by attaching it to the eyepiece of the microscope.

After confirming that beads were tethered to the cover slip, samples were stained with a dilute solution of POPO-3-iodide (534/570) by spreading a small drop of the dye solution on the active region of the cover slip. Staining was performed for no more than 1 hour, followed by gentle but thorough rinsing of the cover slip with Millipore water. Samples were replenished with TETw buffer prior to microscope investigation. Excitation light came from a solid state SUWTECH LDC-1500 laser with 532 emission. Laser and fluorescence emission were directed through an appropriate filter set.

\section{Results and discussion}

Tethered beads. The modified procedure proved successful in tethering beads to the glass cover slip via $\lambda$-DNA. Evidence for this was seen when examining the sample in the absence and presence of a permanent magnet. Without the magnet, beads exhibited Brownian motion that was similar to, but characteristically different from, non-tethered beads; Brownian motion from non-tethered beads is typically more chaotic and usually results in bead diffusion out of the field of view within a few minutes. In the presence of the magnet, beads unambiguously responded by migrating towards the magnet, while becoming more "fixed" in a particular focal plane, as illustrated in Figure 4. Interesting to note is that often there were multiple focal planes separated by a few 
microns that each contained a number of tethered beads. This is most likely due to partial adsorption of the DNA tether to the modified glass substrate, a hypothesis supported by several microscope accounts of watching tethered beads suddenly "pop" out of focus into a higher focal plane, while remaining tethered.

A few experimental parameters still need to be worked out. In particular, density of tethered beads must be reduced to avoid physical "cross talk" between adjacent DNA tethers and beads. Often during microscope inspection, the Brownian motion of two or more beads would randomly become localized in a very small region of the cover slip, suggesting that DNA tethers were entangled. Additionally, when a strong enough field was applied, adjacent beads would quickly aggregate if they were close enough. These issues should be easy to solve simply by determining a more appropriate dilution of reagents.

Video footage. Figure 5 contains three snapshots from a video showing a region of tethered beads and aggregates responding to a permanent magnet. As is evident, too dense coverage of tethered beads results in formation of large chain-like aggregates in the presence of a magnetic field. This undesirable phenomenon is not completely reversible upon removal of magnet. Rather, the aggregates only partially break up. These images illustrate the need to control the density of tethered beads as well as minimize aggregation of beads before attachment to tethers.

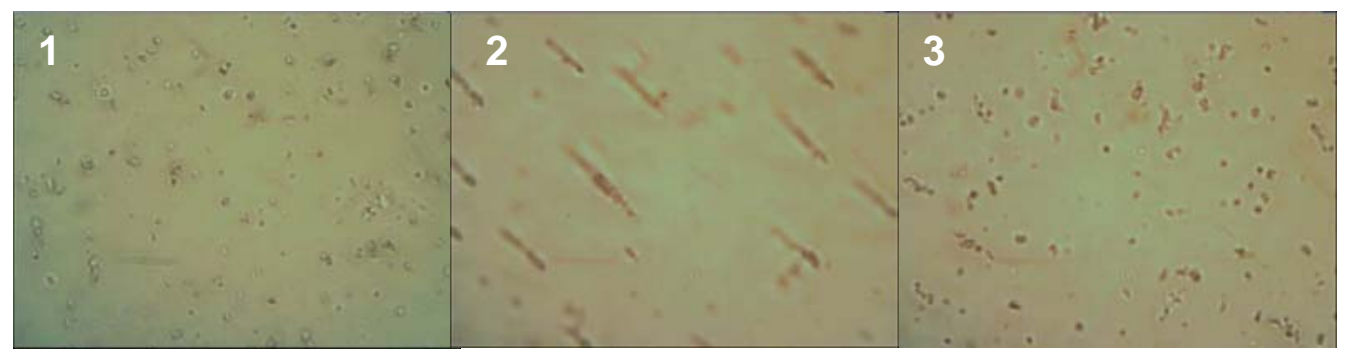

Figure 5. Snapshots of a region of tethered beads and aggregates responding to a permanent magnet. The broad size distribution of beads seen in (1) is attributed to aggregation of beads prior to bead attachment. (1) No magnet; (2) with magnet, beads and aggregates form further chain-like aggregates as they align with and migrate in the direction of the magnetic field; (3) After removing the magnet, aggregates only partially break up. 
Figure 6 shows a representative series of video snapshots of a region on a cover slip where only one tethered bead is present. These particular snapshots were obtained by cropping the full scale video to show only the desired area, and rescaling to fit in the figure. The video clearly shows a single bead migrating towards the permanent magnet as the magnet is moved to various locations around the cover slip sample.

Also shown in Figure 6 are the limits of migration imposed by the $\lambda$-DNA tether. However, it is important to note that the magnet is positioned above at an angle relative to the sample (Figure 4). Consequently, the full length of the DNA tether $(\sim 17 \mu \mathrm{m})$ is not observed as the bead moves from east to west, north to south, etc. A more controlled study is needed to quantify actual distanced traveled by the migrating bead. In particular, stronger and more well-defined magnetic fields need to be used to both subdue the beads against Brownian motion as well as allow more flexibility over the direction of the applied field.

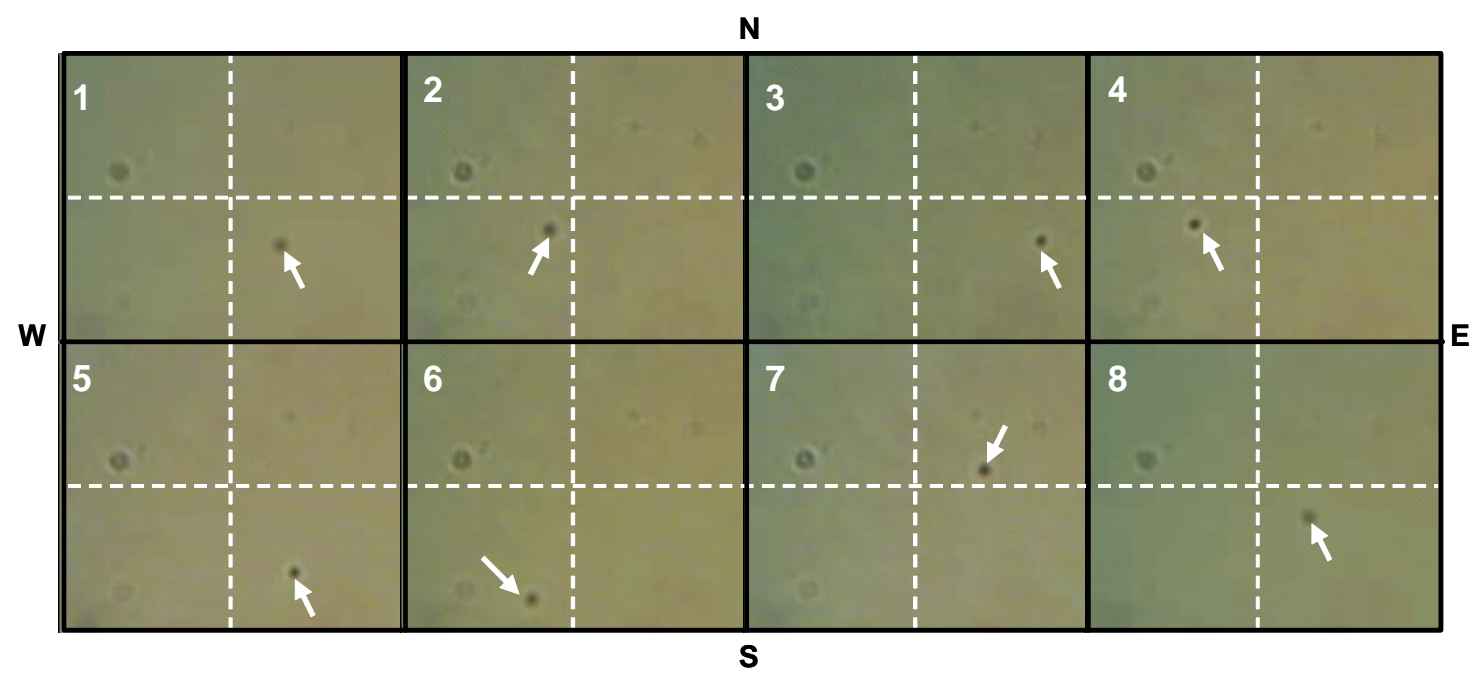

Figure 6. Magnified video snapshots of a single tethered bead (white arrows) responding to a permanent magnet at various locations around the sample. The superimposed white dotted lines divide each frame into four quadrants to aid in following bead location. N, S, $\mathrm{E}, \mathrm{W}$ refer to north, south, east, and west and indicate where the magnet was placed relative to the sample. (1) No magnet; (2) W; (3) E; (4) W again; (5); SE; (6) SW; (7) NE; (8) magnet removed.

Magnetic tweezers. The two custom-built magnetic tweezers devices shown in Figure 3 were initial efforts at constructing low voltage $(<50 \mathrm{~V})$ driven devices, the interest of which arises from safety and heat issues. Higher voltage devices reported in the literature are typically operated at hundreds of volts, and are obviously more dangerous, and generate substantially more heat during operation. Consequently, a "high" voltage device needs to have an effective integrated cooling system and proper safety features to eliminate potential shock hazards.

The two-pole device (Figure 3A) was built with coils designed to withstand $20 \mathrm{~V}$ sustained operating voltage, but could handle brief periods of up to $\sim 85-90 \mathrm{~V}$. Operating at 20-30 V produced negligible heat, in contrast to operating at $85 \mathrm{~V}$, which produced substantial heat and coil damage if driven for too long. Low operating voltages were insufficient at inducing a significant response from a stock suspension of magnetic beads. However, when the voltage was increased to $>80 \mathrm{~V}$, bead suspensions 
responded by aligning and simultaneously forming chain-like aggregates very similar to those shown in the second snapshot of Figure 5. These aggregates grew longer in length with increasing voltage, and could be flipped back and forth as one of the probe voltages was varied while keeping the other constant. In addition to aggregate formation, Brownian motion was significantly attenuated at higher voltages.

An important observation to note is that, in contrast to the permanent magnet, no net migration of the aggregates in the suspension was observed. On the contrary, the aggregates behaved as if being "held" in suspension by a pair of tweezers. As in the case of the permanent magnet, aggregates would nearly completely break up by turning off the voltage. When used on actual samples containing tethered beads, there was minimal bead response to the magnetic field at low voltages, and it was difficult to distinguish this response from Brownian motion. At higher voltages, bead response was almost entirely a function of bead density; at high density where adjacent beads were close enough to touch, aggregation and alignment was observed; at low density, no noticeable response was observed. Again, no net migration of the tethered beads was observed.

The next generation magnetic tweezers device (Figure 3B) addressed the need for higher voltages and greater control over the flux density and direction of the magnetic field by incorporating four poles and larger coils able to withstand a sustained operating voltage of $50 \mathrm{~V}$, though voltages of up to $120 \mathrm{~V}$ were easily withstood for short periods. Bead suspensions and tethered bead samples responded similar to the two-pole case; alignment and chain-like aggregation at high bead density; or no noticeable response at low bead density. As with the two-pole case, no net migration of beads was observed.

When comparing bead response to a permanent magnet versus the multi-pole magnetic tweezers devices, two major differences are clear. 1) A permanent magnet results in a net force vector on the beads towards the magnet, causing migration towards the magnet and eventual tension in the DNA tether. In contrast, the voltage driven device tends to subdue, or "hold" the beads in place, not necessarily applying tension to the DNA tether, and not resulting in a net migration. 2) The voltage driven devices significantly attenuate Brownian motion at higher voltages, while the permanent magnet results in very little, if any, attenuation. It is likely the case that if much higher voltages are used, net migration of beads could be imposed by varying voltages of poles.

Based on these preliminary observations, it appears feasible that with proper probe design considerations (e.g. - probe orientation, spacing between the probes, distance of probes from sample) as well as sufficient characterization of magnetic fields, the use of devices operating below $100 \mathrm{~V}$ will suffice in producing bead motion suitable for SMS experiments.

Fluorescence measurements. The method used for staining DNA tethers resulted in levels of background fluorescence unacceptable for single molecule studies, which require critically low background for acceptable signal to noise ratio. A typical post-staining observation showed a faint, uniform, visually observable fluorescence emission across the cover slip surface focal plane, with fluorescing DNA tethers and magnetic beads dispersed across the field of view at higher focal planes. Cover slips modified only with oligo (no DNA or beads) also showed the same uniform fluorescence at the cover slip surface focal plane when stained in an identical manner to the actual sample. Furthermore, unmodified cover slips showed no fluorescence upon staining. From these control studies, it was obvious that the fluorescent stain was adsorbing or chemically binding to DNA tethers, bead surfaces, as well as areas of the modified glass surface with no DNA tethers. There was insufficient time in the 10-week period to 
address this undesirable issue. However, it should not be difficult to prepare a sample where only the DNA tether is stained.

It is important to note that if salt deposits left during evaporation of sample in Step 2 of sample preparation are not sufficiently washed away, they will serve as a significant source of background fluorescence as well. In a previous method, samples were evaporated to dryness at $50^{\circ} \mathrm{C}$, cured for 5 minutes at the same temperature, and subsequently rinsed in hot water. As a result, salt deposits became "etched" into the glass cover slip, much as hard water spots can etch into glass surfaces, and would not dissolve or otherwise wash away in the hot water wash. Microscope inspection showed bright red salt crystals under laser excitation, both with and without sample staining.

\section{Concluding remarks}

The integration of single molecule fluorescence measurements on a molecule under physical control by magnetic tweezers would represent a significant step in the field of single molecule spectroscopy. Coupling these two techniques is ideal since magnetic fields do not produce perturbations that will interfere with fluorescence measurements. Furthermore, the experimental details of each technique are well developed and well suited for the study of biomolecular conformational dynamics, a core area of interest in Peter Lu's research agenda.

The 10 week summer internship afforded sufficient time to make notable progress towards the research goal. After trialing through a number of sample prep methods, a working procedure that is both consistent and well suited for future single molecule experiments materialized, though a few experimental details are in need of revision. Despite not achieving all three goals as described in the Research Summary, all indications are that coupling SMS with magnetic tweezers is feasible at PNNL.

Lastly, I wish to express my personal satisfaction with the summer program, the research, the mentoring, and the overall experience. Everyone involved made me feel welcome and valuable. I was treated with the highest level of respect and professionalism, while at the same time welcomed into informal discussions and conversations on everything from science to socialism. From a career standpoint, this program resulted in personal contacts that will certainly be of value after graduating with my Ph.D. As there is potential for collaborations between my advisor, Alex Li, and Peter Lu, I look forward to the opportunity to visit PNNL again. 


\section{Acknowledgements}

I would like to thank the following people at PNNL:

Dr. Steve Barlow, Chris Montgomery, and Rebecca Janosky for making this program possible.

Dr. Peter Lu for providing an opportunity to work in his lab, and his excellent mentorship, guidance, and support.

Dr. Vasudevan Pillai Biju, Dr. DeHong Hu, Dr. Duohai Pan, Dr. Serdar Ozcelik and Dr. $\quad$ Gary Holton for technical help and stimulating discussions and conversations.

Beverly in IDL for building magnetic tweezers

Dr. Wayne Hess for sanity through cycling.

\section{References}

1) X. Tan, P. Nalbant, A. Toutchkine, D. Hu, E. R. Vorpagel, K. M. Hahn, and H. P. $\mathrm{Lu}$, "Single-Molecule Study of Protein-Protein Interaction Dynamics in a Cell Signaling System," J. Phys. Chem. B., 108, 737-744 (2004).

2) H. P. Lu, L. M. lakoucheva, and E. J. Ackerman, "Single-Molecule Conformational Dynamics of Fluctuating Noncovalent DNA-Protein Interactions in DNA Damage Recognition," J. Am. Chem. Soc., 123, 9184-9185 (2001).

3) Zlatanova, J.; Leuba, S.H., "Magnetic tweezers; a sensitive tool to study DNA and chromatin at the single-molecule level," Biochem. Cell Biol., 81, 151-159 (2003)

4) Haber, C.; Wirtz, D., "Magnetic tweezers for DNA manipulation," Rev. Sci. Instrum., 71(12), 4561-4570 (2000)

5) Strick, T.R.; Croquette, V.; Bensimon, D., "Homolgous pairing in stretched supercoiled DNA," Proc. Natl, Acad. Sci USA, 95, 10579-10583 (1998)

6) Weiss, S., "Fluorescence Spectroscopy of Single Biomolecules," Science, 283, 1676-1683 (1999)

7) Kumar, A.; Larsson, O.; Parodi, D.; Liang, Z., "Silanized nucleic acids: a general platform for DNA immobilization," Nucleic Acids Res., 28(14), e71 (2000)

8) Rogers, Y-H.; Jiang-Baucom, P.; Huang, Z-J.; Bogdanov, V.; Anderson, S.; Boyce-Jacino, M.T., "Immobilization of Oligonucleotides onto a Glass Support via Disulfide Bonds: A Method for Preparation of DNA Microarrays," Anal. Biochem., 266, 23-30 (1999) 


\title{
The spectroscopic signature of the transition from "all- surface" to "internally solvated" structures in water clusters
}

\author{
Anita Lagutschenkov and Gereon Niedner- Schatteburg \\ Department of Chemistry \\ Technical University of Kaiserlautern \\ Kaiserlautern, Germany \\ and \\ Sotiris S. Xantheas \\ Pacific Northwest National Laboratory \\ Richland, WA
}

The structural patterns adopted by the clusters of the first few molecules of water are determined from the maximization of hydrogen bonding, the minimization of dangling bonds and the formation of local networks, which exhibit large cooperative effects. An informative probing of these hydrogen bonding networks is achieved by infrared (IR) spectroscopy in the $3000-4000 \mathrm{~cm}^{-1}$ range, a part of the spectra that corresponds to the "fingerprint" of the underlying network and its connectivity. Theory does provide the missing link in the correspondence between experimental spectra and underlying structure. It is of interest to investigate the structural transition between "allsurface" and "internally solvated" structures since this will provide the cluster regime which mimics the fully solvated environment of a water molecule in condensed phase. Earlier studies ${ }^{1}$ based on evolutionary algorithms using the TIP4P (Ref. 2) and TTM2-F (Ref. 3) empirical interaction potentials for water identified a size-dependent transition from "all-surface" (all molecules on the surface of the cluster) to "internally solvated" (one water molecule at the center, fully solvated) structures starting at cluster size $n=17$. At the same time the two empirical potentials used (TIP4P and TTM2-F) produced different qualitative results as regards both the onset and the structural motif of the clusters involved. In particular, for $n=17$ the TIP4P potential produced the "all surface" structure as the lowest energy whereas the TTM2-F yielded the "interior" structure as more stable. For $n=21$ TIP4P produced an "all surface" global minimum structure whereas the TTM2-F an "interior" structure as the most stable one. The TTM2-F interaction potential has been parametrized from high level ab-initio electronic structure calculations on small $(n=2-6)$ water clusters ${ }^{4}$ and was found to reproduce the MP2/CBS (Complete Basis Set) limit binding energies of larger clusters such as the $\operatorname{octamer}^{5}(n=8)$ and various isomers of the ecosamer ${ }^{6}(n=20)$ to within an impressive $(<1 \mathrm{kcal} / \mathrm{mol}$ or $<0.5 \%$ ) accuracy, far better than any other interaction potential for water. Given the fact that it produces a different trend than TIP4P for this structural transition, it is of interest to investigate this effect from first principles electronic structure calculations.

We have performed geometry optimizations at the density functional ${ }^{7}$ (DFT) and second order Møller-Plesset perturbation ${ }^{8}$ (MP2) levels of theory starting from the minimum energy structures with the TIP4P and TTM2-F interaction potentials ${ }^{1}$ for the cluster regime $n=17-21$. These structures are shown in Figure 1. We employed the B3LYP functional ${ }^{9}$ and the Ahlrichs TZVP orbital basis set ${ }^{10}$ in the DFT calculations and the aug-cc-pVDZ and aug-cc-pVTZ basis sets ${ }^{11}$ for the MP2 calculations. All structures were reoptimized in both the DFT and MP2 calculations with all basis sets. Harmonic 
frequency analysis was performed at the optimal B3LYP/TZVP and selected MP2/augcc-pVDZ geometries. All calculations were carried out using the NWCHEM suite of codes on the newly acquired $1900+$ processor massively parallel Hewlett-Packard supercomputer at the Molecular Science Computing Facility in the William R. Wiley Environmental Molecular Sciences Laboratory at Pacific Northwest National Laboratory. The 11.4 Teraflop supercomputer consists of $19001.5 \mathrm{GHz}$ Intel Itanium 2 processors coupled together with Quadrics interconnect. The system has 7 Terabytes of memory and over $1 / 2$ Petabyte of total disk storage, making it a uniquely balanced resource for computational chemistry.

Figure 1 suggests that the TIP4P and TTM2-F potentials both yield "all-surface" global minima for $n=18$ and 20 and "internally solvated" global minima for $n=19$. For $n=17$ and 21 the TIP4P potential produces the "all-surface" minimum as the lowest energy one where the situation is reversed with the TTM2-F potential which suggests that the "internally solvated" minimum is the one of lowest energy. The results with the 2 potentials as well as with the ab-initio calculations are summarized in Table 1. It is readily seen that both the B3LYP and MP2 with the various basis sets produce results that are consistent with the predictions of the TTM2-F potential for $n=17$ and 21, i.e. suggesting that the "internally solvated" minimum is lower in energy than the "allsurface" one. They furthermore suggest that the energy difference between the two configurations is well reproduced with the TTM2-F potential.

It is of further interest to investigate the difference in the IR spectra between the two differently distinct networks in order to provide valuable information that can be used for the experimental assignment for the onset of this structural transition. The harmonic frequency analysis for the global minima of the $n=17-21$ clusters has been performed at the DFT (B3LYP/TZVP) level of theory. Figure 2 shows the comparison between the MP2/aug-cc-pVDZ and B3LYP/TZVP calculated spectra (produced using a Lorentzian line shape with a uniform $10 \mathrm{~cm}^{-1}$ half-width at half-maximum) for the global minimum of $n=20$ in the $3000-4000 \mathrm{~cm}^{-1}$ range. We note that the spectral pattern (relative positions and intensities) remains the same between the 2 methods with DFT yielding IR active bands that are in general more red-shifted than MP2. For the most redshifted $\mathrm{OH}$ stretching band this difference is ca. $80 \mathrm{~cm}^{-1}$. The IR spectra of the global minima for the clusters $n=17-21$ are shown in Figure 3(a)-(e). It is readily seen that in general "internally-solvated" configurations for $n=17,19,21$ (Figure 2, panels (a), (c), (e)) are associated with spectral patterns that exhibit more red shifted $\mathrm{OH}$ stretching bands than "all-surface" configurations for $n=18,20$ (Figure 2 panels (b) and (d)). The alternation between "internally-solvated" and "all-surface" configurations from $n=17-21$ is also reflected in the alternation of spectral patterns in the corresponding IR spectra.

In conclusion we have used electronic structure calculations in order to confirm that the structural transition between "all-surface" and "internally-solvated" structures in water clusters occurs at cluster size $n=17$ as predicted by the TTM2-F empirical interaction potential. The calculated IR spectra in the $3000-4000 \mathrm{~cm}^{-1}$ range can offer useful guidance for the experimental verification of this transition using vibrational spectroscopy. 


\section{References}

1. B. Hartke, Phys. Chem. Chem. Phys. 5, 275 (2003).

2. W. L. Jorgensen, J. Chandrasekhar, J. D. Madura, R. W. Impey and M. L. Klein, J. Chem. Phys. 79, 926 (1983).

3. C. J. Burnham and S. S. Xantheas, J. Chem. Phys. 116, 5115 (2002).

4. S. S. Xantheas, C. J. Burnham, R. J. Harrison, J. Chem. Phys. 116, 1493 (2002).

5. S. S. Xantheas, E. Apra, J. Chem. Phys. 120, 823 (2004).

6. G. S. Fanourgakis, E. Apra and S. S. Xantheas, J. Chem. Phys. 121, 2655 (2004).

7. P. Hohenberg and W. Kohn, Phys. Rev. 136, B864 (1964).

8. C. Møller and M. S. Plesset, Phys. Rev. 46, 618 (1934).

9. A. D. Becke, J. Chem. Phys. 98, 5648 (1993).

10. A. Schäfer, C. Huber, and R. Ahlrichs, J. Chem. Phys. 100, 5829 (1994).

11. T. H. Dunning, Jr., J. Chem. Phys. 90, 1007 (1989); R. A. Kendall, T. H. Dunning, Jr., and R. J. Harrison, J. Chem. Phys. 96, 6796 (1992).

12. T. P. Straatsma, E. Aprà, T. L. Windus, M. Dupuis, E. J. Bylaska, W. de Jong, S. Hirata, D. M. A Smith, M. Hackler, L. Pollack, R. Harrison, J. Nieplocha, V. Tipparaju, M. Krishnan, E. Brown, G. Cisneros, G. Fann, H. Fruchtl, J. Garza, K. Hirao, R. Kendall, J. Nichols, K. Tsemekhman, M. Valiev, K. Wolinski, J. Anchell, D. Bernholdt, P. Borowski, T. Clark, D. Clerc, H. Dachsel, M. Deegan, K. Dyall, D. Elwood, E. Glendening, M. Gutowski, A. Hess, J. Jaffe, B. Johnson, J. Ju, R. Kobayashi, R. Kutteh, Z. Lin, R. Littlefield, X. Long, B. Meng, T. Nakajima, S. Niu, M. Rosing, G. Sandrone, M. Stave, H. Taylor, G. Thomas, J. van Lenthe, A. Wong, Z. Zhang, "NWChem, A Computational Chemistry Package for Parallel Computers, Version 4.5" (2003), Pacific Northwest National Laboratory, Richland, Washington 99352-0999, USA; R. A. Kendall, E. Aprà, D. E. Bernholdt, E. J. Bylaska, M.

Dupuis, G. I. Fann, R. J. Harrison, J. Ju, J. A. Nichols, J. Nieplocha, T. P. Straatsma, T.L. Windus, A.T. Wong, Computer Phys. Comm. 128, 260 (2000).

13. For more information refer to http://www.pnl.gov/news/2002/computer.htm and http://www.emsl.pnl.gov/capabs/mscf/. 
Table 1. Energy separation between "all-surface" and "interior" configurations for $n=17$ and 21 with the various empirical potentials and electronic structure methods.

\begin{tabular}{ccccccc}
\hline \hline \multirow{2}{*}{$\begin{array}{c}\text { Cluster } \\
\text { size }\end{array}$} & Configuration & \multicolumn{5}{c}{$\Delta \Delta E)(\mathrm{kcal} / \mathrm{mol})$} \\
\cline { 3 - 7 }$N$ & & TIP4P & TTM2-F & $\begin{array}{c}\text { B3LYP/ } \\
\text { TZVP }\end{array}$ & $\begin{array}{c}\text { MP2/aug-cc- MP2/aug-cc- } \\
\text { pVDZ }\end{array}$ & pVTZ \\
\hline \multirow{2}{*}{17} & all-surface & 0 & 1.11 & 1.76 & 1.73 & 1.43 \\
& interior & 0.5 & 0 & 0 & 0 & 0 \\
21 & all-surface & 0 & 1.81 & 6.92 & 4.27 & 3.71 \\
\hline \hline
\end{tabular}




\section{"all-surface"}
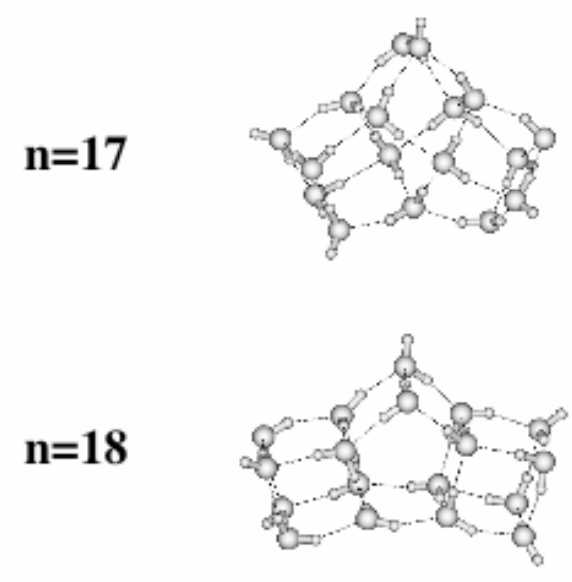

$n=19$
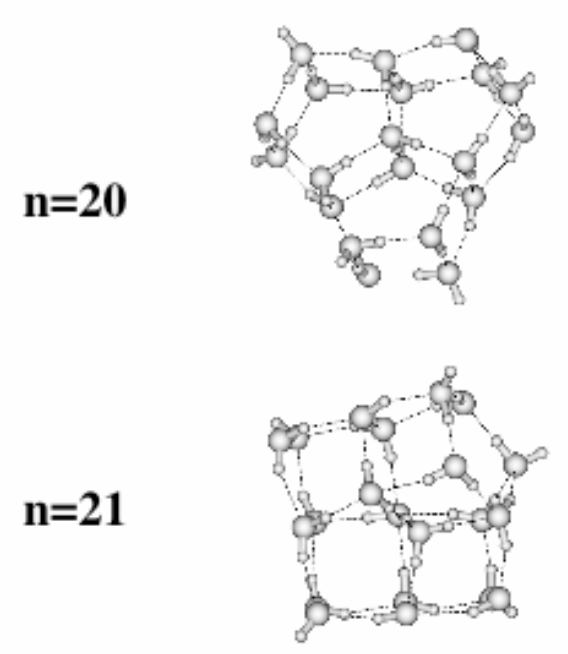

\section{"interior"}
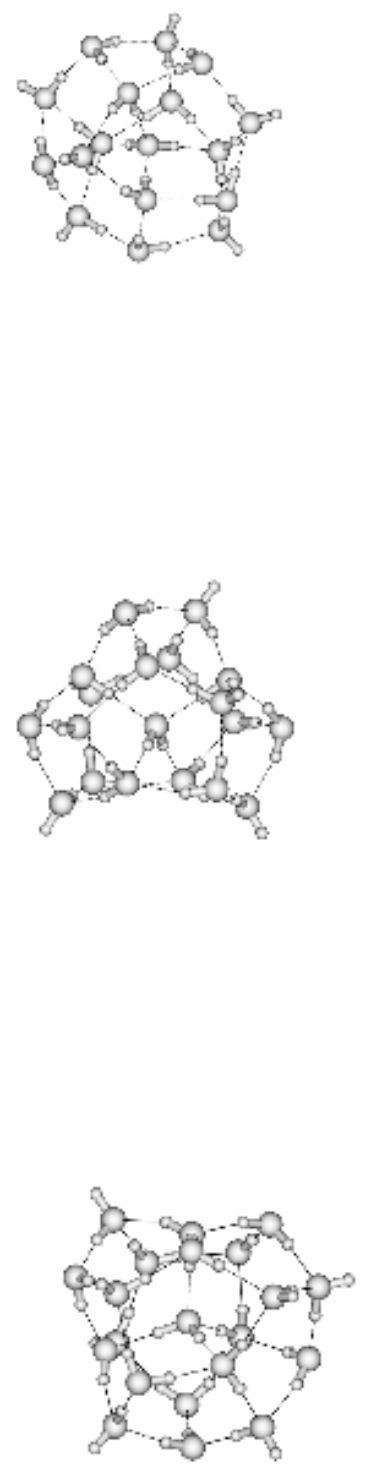

Figure 1. Minimum energy structures for the "all-surface" and "internally solvated" configurations for $n=17,21$. For $n=18-20$ the global minimum structures are indicated. 


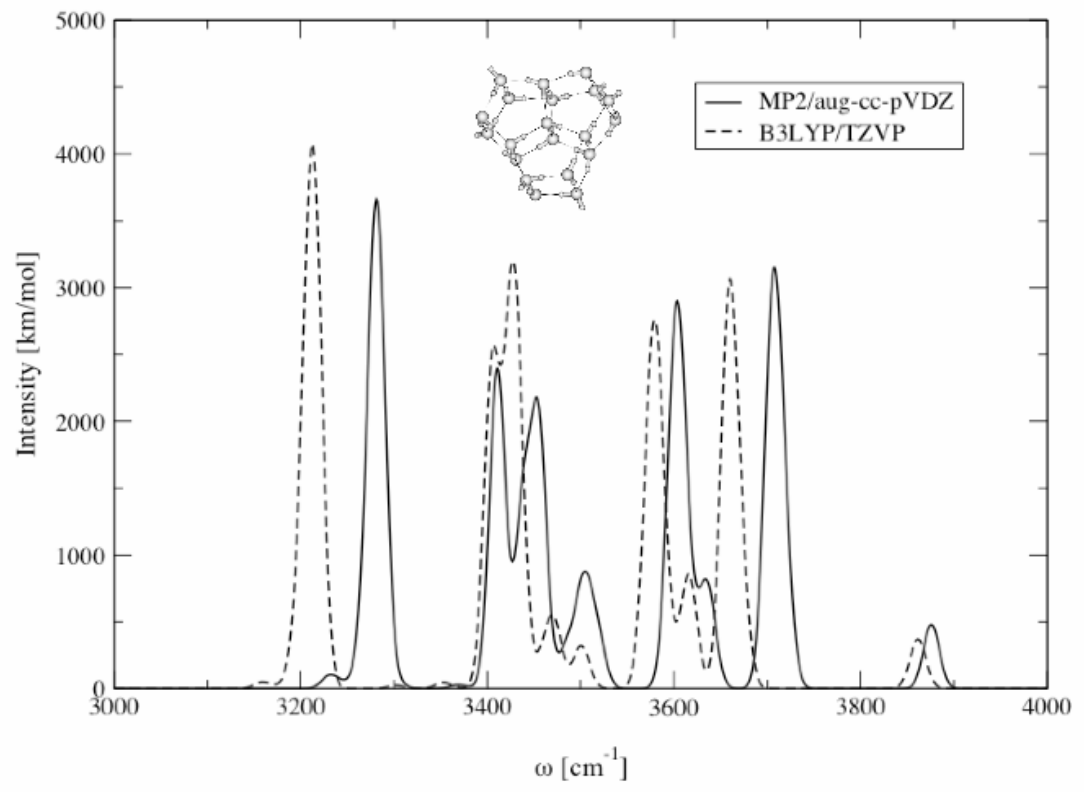

Figure 2. Comparison of the B3LYP and MP2 IR spectra in the $3000-4000 \mathrm{~cm}^{-1}$ range for the global minimum of $\left(\mathrm{H}_{2} \mathrm{O}\right)_{20}$. 


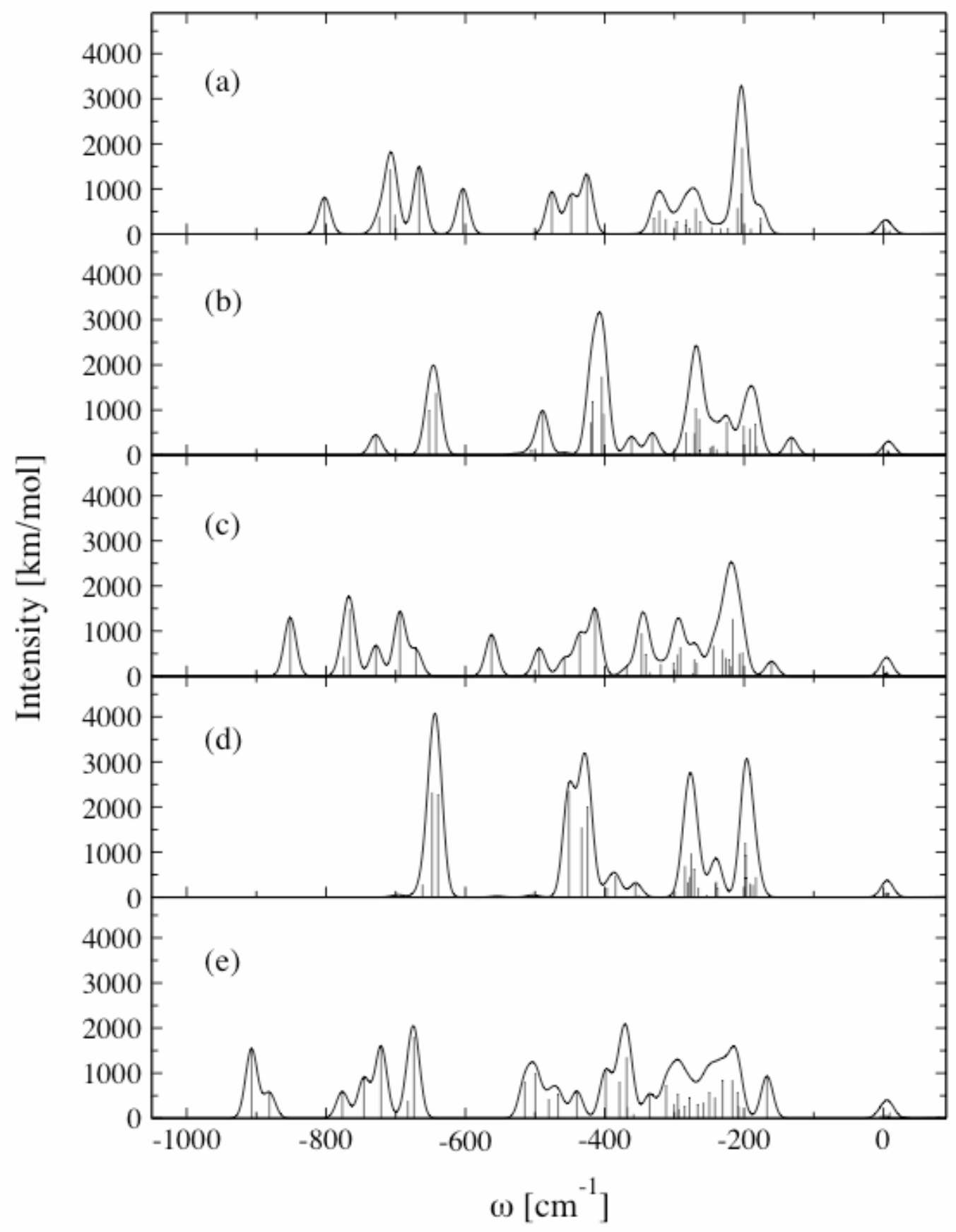

Figure 3. Calculated infrared (IR) vibrational spectra for the global minima for $n=17-21$, (a)-(e). 


\title{
Measurement of charge carrier lifetimes in $\mathrm{Fe}_{2} \mathrm{O}_{3}$ thin films
}

\author{
David M. Laman \\ Physics Department \\ University of Central Washington \\ Ellensburg, WA \\ And \\ Alan Joly \\ Pacific Northwest Laboratory \\ Richland, WA
}

\begin{abstract}
Charge carrier lifetime measurements in $\mathrm{Fe}_{2} \mathrm{O}_{3}$ thin films were carried out via pumpprobe transient absorption and transient reflectivity. Excitation into the conduction band of the film was provided by the 150 fs pulses of a frequency doubled Ti-Sapphire laser, and the charge carrier population was probed with a wavelength selected white light source created by focusing the fs near-IR pulses of the Ti:Sapphire laser into a cell of water. Charge carrier decay curves were obtained for a variety of probe beam wavelengths in order to aid in determining the contribution of ground state absorption to the observed decay curves. Decay curves were also obtained at different points on the films in order to determine if spatial inhomogeneities might affect the observed decay kinetics. The decay curves obtained appear to rapidly decay at early times $(<1 \mathrm{ps})$ and slowly decay at later times ( $>1 \mathrm{ps})$, and thus we are currently fitting a bi-exponential kinetic model to the decay curves in order to extract decay rate constants.
\end{abstract}




\title{
Atomistic Modeling of Helium Clustering and Diffusion along Dislocations in Alpha-Iron
}

\author{
Edward A. Le and Lucien Brush \\ Material Science \& Engineering \\ University of Washington \\ Seattle, WA \\ And \\ Charles Henager \\ Pacific Northwest National Laboratory \\ Richland, WA
}

\begin{abstract}
Molecular statics and molecular dynamics are being used to study the fate of helium in the vicinity of $a / 2<111>\{110\}$ edge dislocations and $a / 2<111>$ screw dislocations in alpha-iron. In the present study binding energies of interstitial He atoms, substitutional He atoms and He-divacancy complexes, respectively, to the dislocations were determined. Interstitial He atoms can migrate and be trapped near the center of dislocations with binding energies of $\sim 0.5 \mathrm{eV}$ to the screw dislocation and $\sim 1.2 \mathrm{eV}$ to the edge dislocation. Clustering of interstitial $\mathrm{He}$ atoms and substitutional $\mathrm{He}$ atoms in the vicinity of the dislocations was also examined. Interstitial and substitutional $\mathrm{He}$ atoms can form stable clusters depending on their positions relative to the dislocations.
\end{abstract}

\section{Introduction}

During the operation of fusion reactors, He is directly implanted or generated internally by nuclear transmutation reactions in materials, concurrently with energetic displacement damage [3]. The creation of helium atoms in metals is considered with particular concern since their precipitation into bubbles can substantially deteriorate mechanical properties, particularly in metals at high temperature, $\mathrm{T} \geq 0.5 \mathrm{~T}_{\mathrm{m}}$, where drastic embrittlement of metals due to helium bubbles formation at grain boundaries and dislocation lines has been found to occur even at very low overall helium concentrations [2]. In the present study, molecular statics and molecular dynamics are employed to study the binding of $\mathrm{He}$ interstitials and substitutional atoms to dislocations, and the interaction of $\mathrm{He}$ atoms to form clusters in $\alpha$-Fe near both edge and screw dislocations. The Ackland potential [5], the Wilson-Johson potential [6] and the Beck potential [7] are employed to describe the interactions of $\mathrm{Fe}-\mathrm{Fe}, \mathrm{Fe}-\mathrm{He}$ and $\mathrm{He}-\mathrm{He}$, respectively.

\section{Dislocation Structure and Simulation Method}

In this research we study the dislocation using a cell with cylindrical geometry. Figure 1 a) shows the orientation of the computational cell and the relative positions of the compressive and tensile strain fields of the edge dislocation. Similarly, in screw 
dislocation the Burgers vector is located along the z-axis and there are none of the compression and tension region at Figure $1 \mathrm{~b}$ ).

Calculations were performed for He atoms placed at crystallographically equivalent interstitial positions along a line normal to the dislocation slip plane. This was done along a vertical line running through the center of the dislocation for edge dislocation calculations and horizontally for screw dislocation calculation. Calculations also were performed for He-divacancy complexes in and near dislocations and clusters formation for both screw and edge dislocation.

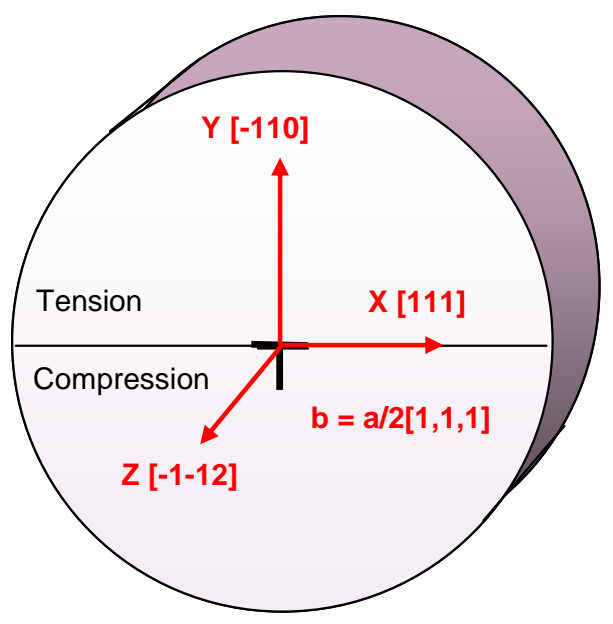

a) a/2[111][-1-12] Edge Dislocation

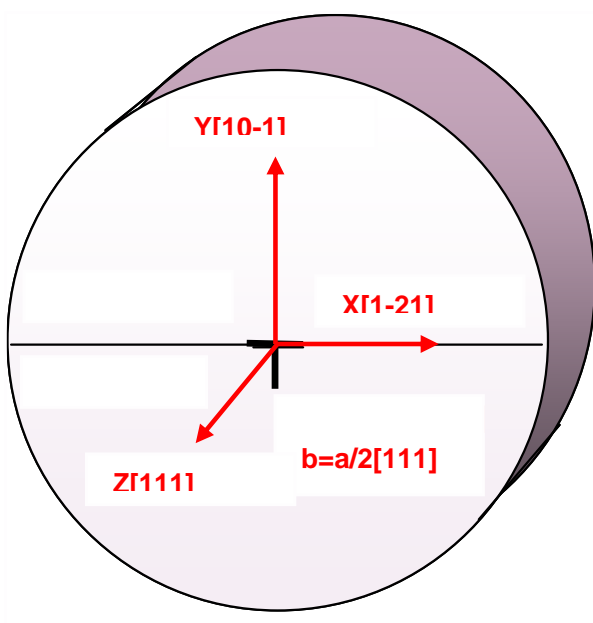

b) a/2[111] Screw Dislocation

Figure 1. Cylindrical computational cell for an a) a/2 [111] [-1-12] edge dislocation and b) a/2[111] screw dislocation in $\alpha$-Fe.

\section{Results}

\subsection{He-divacancy complex and crowdion}

In order to investigate the core energy and the structure of both the edge and screw dislocation in the presence of $\mathrm{He}$ in Fe, we build a spherical cylindrical cell and impose fix boundary conditions. The model contain of a main region, which is the movable region that we consider and there also non-moveable region that account for the fixed boundary condition. 


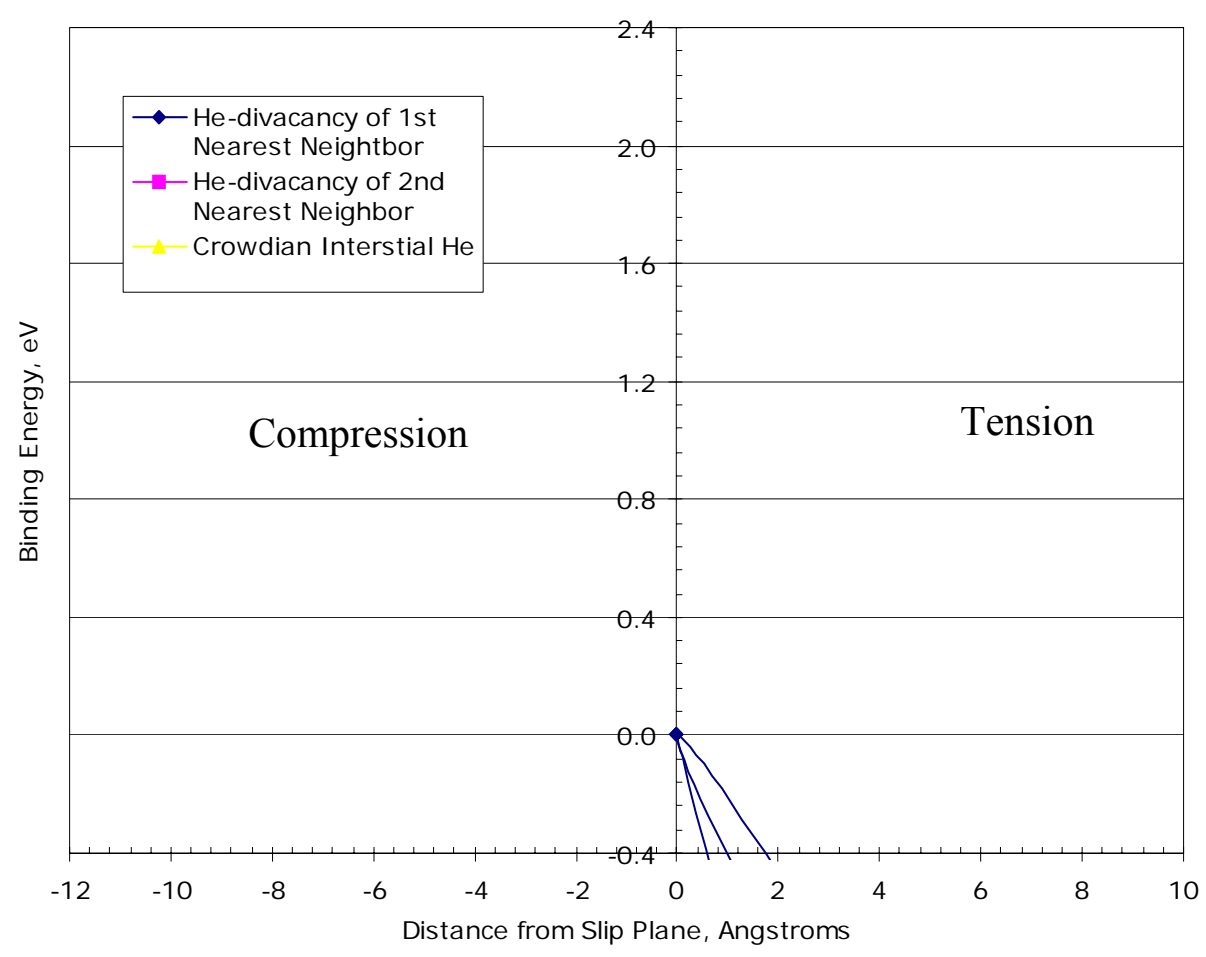

Figure 2. Binding energy of He-divacancy complex and crowdion interstitial.

As shown in Figure 2, relaxation calculations have also been performed to determine binding energy of He-divacancy complexes near the dislocation. The binding energy is the difference between the formation energy of the He-divacancy complexes in the perfect lattice and in its relaxed position near the dislocation. The He-divacancy complexes contain a He substitution and a neighbor vacancy. This defect is essentially a divacancy with a He atom trapped in it. This complex migrates by a mechanism similar to single vacancy migration while dragging the He along. The calculations have been performed for the first nearest neighbor vacancy and second nearest neighbor vacancy. The binding energy is higher for the first nearest neighbor vacancy for the He-divacancy complex. The binding energy is also higher in the tension side of the cylinder in edge dislocation for this He-divacancy complex for both first and second nearest neighbor. As expected, the binding energy is negative (repulsive) in the compressive field of the dislocation and positive (attractive) in the tensile file. This is shown that the He interstitial is easier to get trap and move toward the center core when placed in the tension side. On the other hand, the compression side is not energy stable for He- 
divacancy complex to get trap or move toward dislocation line when placed on the compression side.

Calculations have also been performed to determine binding energy for the crowdion interstitial He across the dislocation. As shown in Figure 2, the binding energy is higher when interstitial $\mathrm{He}$ is placed at $2 \mathrm{~A}^{0}$ or closer to the dislocation. This also implied that all the interstitial He when placed close to the dislocation is become crowdion interstitial.

\subsection{Clusters Formation}

Calculations have been performed to determine formation and binding energies of $\mathrm{He}$ clusters formation for both edge and screw dislocation. In edge dislocation, 4-interstitial He atoms where as two atoms are placed above atom row and two atoms are placed below atom row is relaxed for cluster formation. The distance between two atoms in horizontal direction are $\sim 1.4 \mathrm{~A}^{0}$ apart. The distance between tow atoms in vertical direction are $\sim 2.03 \mathrm{~A}^{0}$ apart. These four atoms are located on the same plane. The 4interstitial $\mathrm{He}$ in screw dislocation is also located on the same plane but $\sim 2.39 \mathrm{~A}^{0}$ apart in vertical direction and $\sim 2.4 \mathrm{~A}^{0}$ apart in horizontal direction. Starting with the same increment and go through the dislocation line the formation of cluster is studied for edge and screw dislocation. As shown in Figure 3, the binding energy is $\sim 4 \mathrm{eV}$ higher for edge dislocation than screw dislocation. Energy also is higher for interstitial cluster that is located near the dislocation line.

Cluster formations also have been studied for the substitution He. In Figure 4, three substitutional $\mathrm{He}$ atoms are also relaxed for the cluster formation. As shown in Figure $4 \mathrm{~b}$ ), there is no cluster formation for the 3 -substitution He atom when there is $\sim 6.1 \mathrm{~A}^{0}$ away from the dislocation. However, cluster is formed at Figure 4 a) when they place $\sim 4.04 \mathrm{~A}^{0}$ away from the dislocation. The energy is $\sim 2.24 \mathrm{eV}$ lower for the case of cluster formed. The same configuration is also studied at the compression side. The results shown that there is no cluster formation when placed in the compression region of the dislocation. 


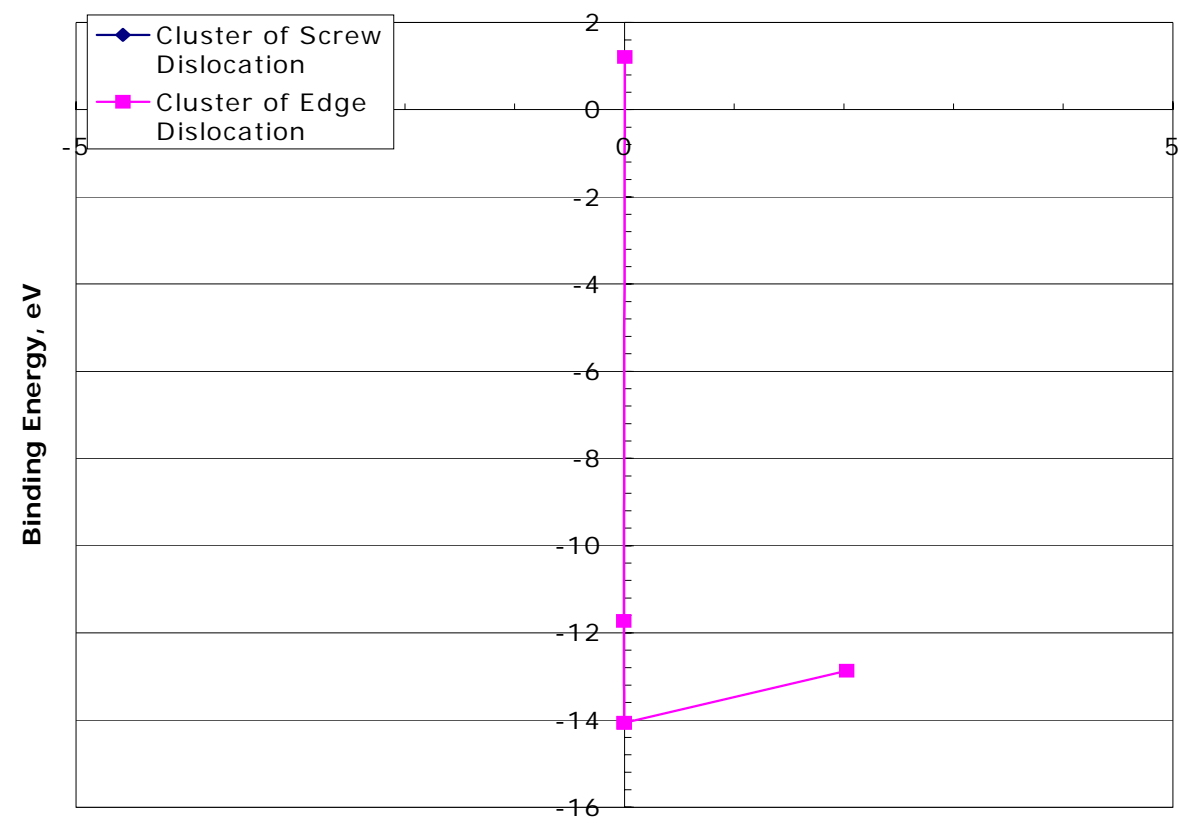

Distance from Slip Plane, Angstroms

Figure 3. Binding energy of clusters of 4-insterstitial He in edge and screw dislocation.

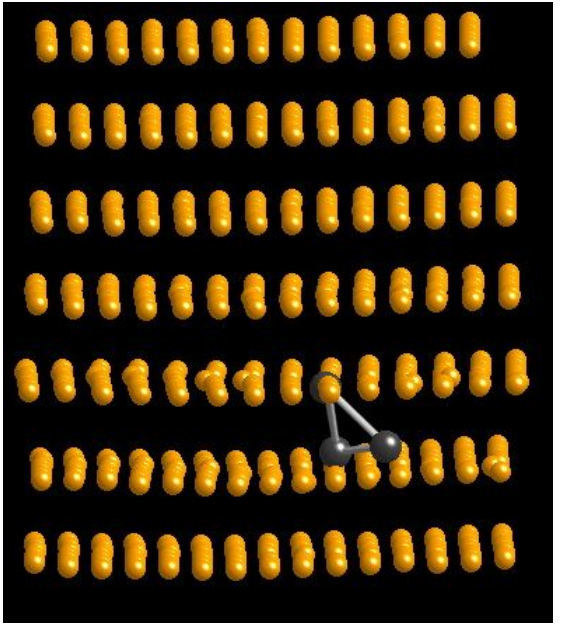

a)

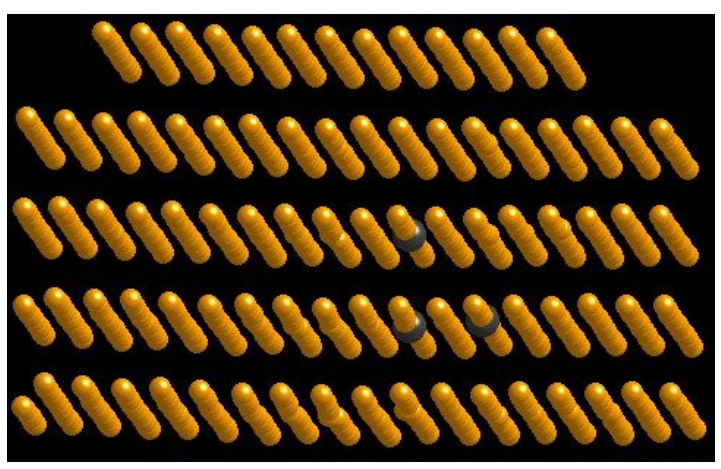

b)

Figure 4. Cluster formation for substitution He at location near dislocation line for edge dislocation when a) 4.04 $\mathrm{A}^{0}$ away from the dislocation line, and b) substitution He atoms at $\sim 6.1 \mathrm{~A}^{0}$ away from the dislocation line. 


\subsection{He interstitial and He-divacancy complex in screw dislocation}

In Figure 5, binding energy is plotted versus distance in $\mathrm{A}^{0}$ from split plane for tetrahedral interstitial $\mathrm{He}$ and octahedral interstitial He. It had been shown that, tetrahedral interstitial He having higher binding energy. Interstitial He with initial tetrahedral position stayed in tetrahedral position. Interstitial He with initial octahedral position go to the tetrahedral position when located $\sim 4.1 \mathrm{~A}^{0}$ away or closer to the dislocation. This indicated that near the dislocation, the tetrahedral position is the more stable configuration. Similar to the edge dislocation, binding energy is lower for atom that is located farther from the dislocation. As results, the farther from the dislocation line the lower the binding energy for substitutional and interstitial $\mathrm{He}$ atoms.

Calculations have also been performed to determine formation and binding energies of He-divacancy complexes for screw dislocation. This defect is essentially a divacancy with a He atom trapped in it. Similar to the edge dislocation, this complex migrates by a mechanism similar to single vacancy migration while dragging the He along.

Calculations have been performed with this complex along a line through the dislocation. In Figure 6, the binding energy of He-divacancy is plotted versus distance from the dislocation plane. He-divacancy with the first nearest neighbor had a higher binding energy than He-divacancy with the second nearest neighbor.

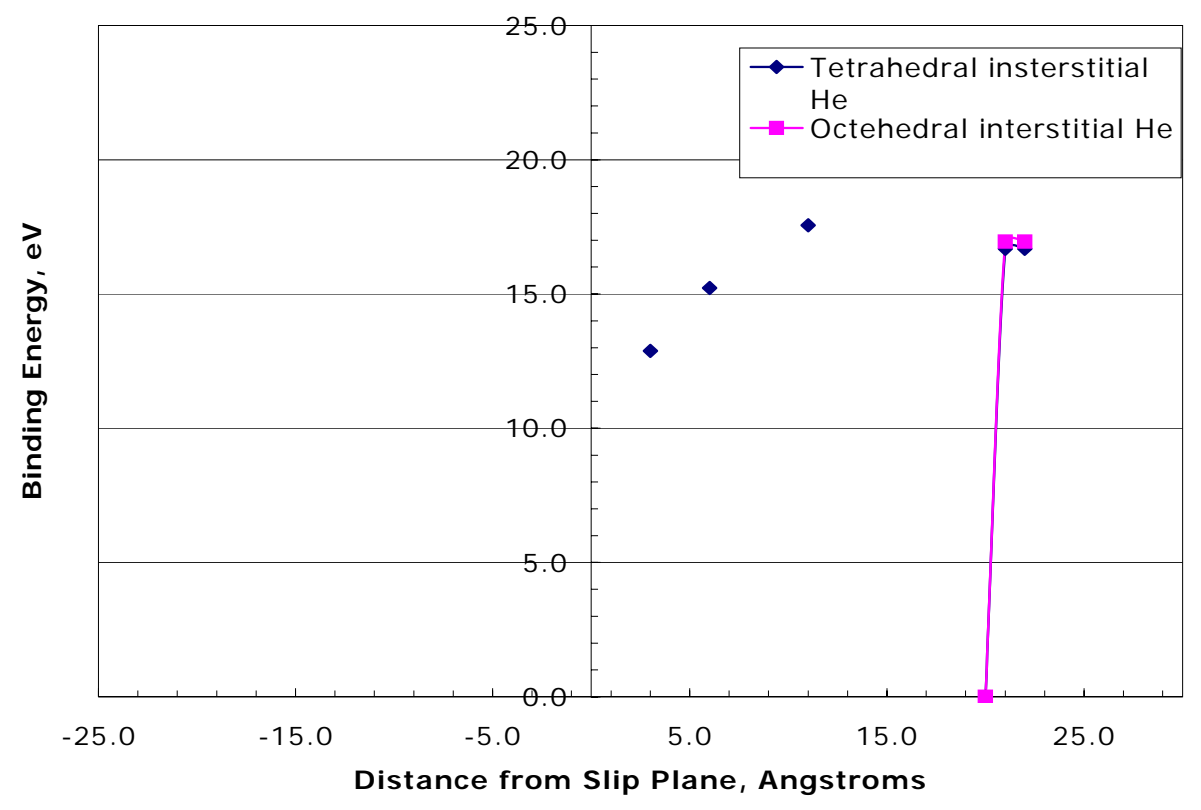

Figure 5. Binding energy of interstitial $\mathrm{He}$ at tetrahedral and octahedral position for screw dislocation. 


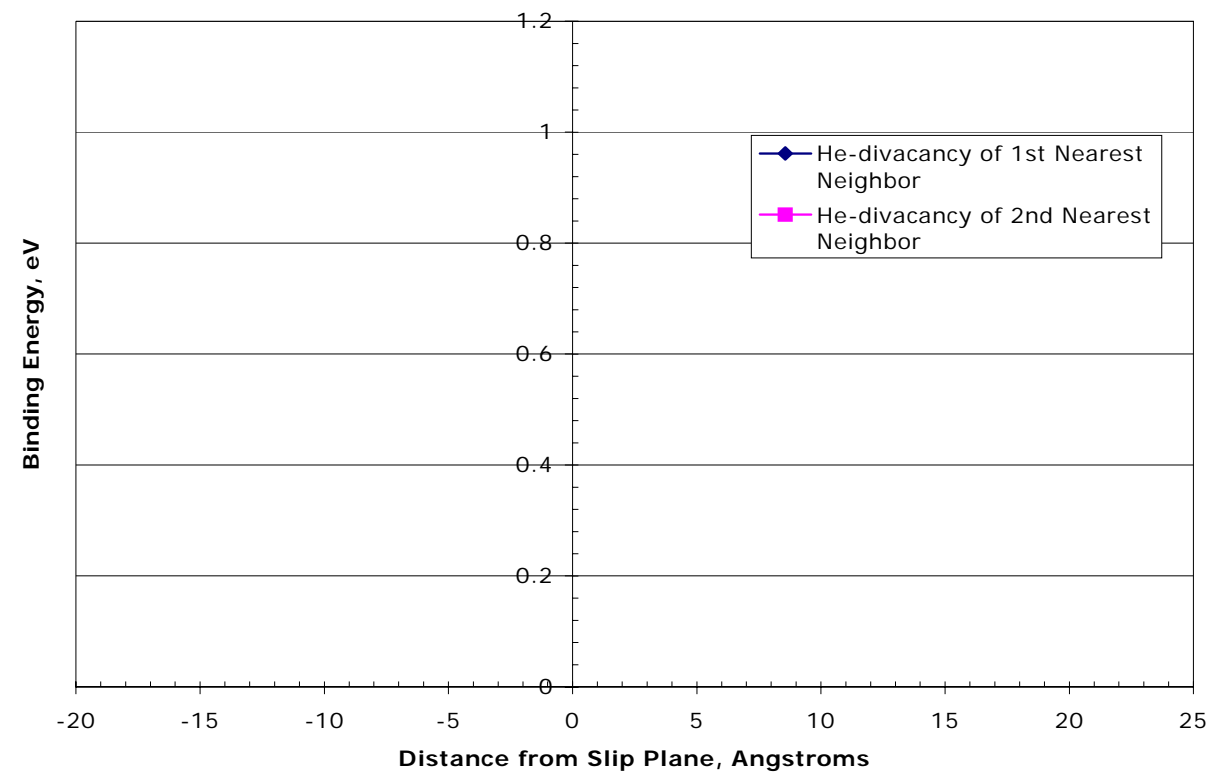

Figure 6. Binding energy of He-divacancy of $1^{\text {st }}$ and $2^{\text {nd }}$ nearest neighbor for screw dislocation.

\section{Summary}

Helium-dislocation interactions in $\alpha$-Fe have been studied for substitutional and interstitial He atoms as well as a He-divacancy complex for both screw and edge dislocations. Cluster formation of both interstitial He and substitutional He near dislocations was also studied. An interstitial He with initial tetrahedral position stayed in tetrahedral position. Interstitial He with initial octahedral position relaxed to the tetrahedral position when located $\sim 4.1 \mathrm{~A}^{0}$ away or closer to the edge dislocation. The binding energy is negative in the compressive field of the dislocation and positive in the tensile file for edge dislocation. He-divacancy complexes with the first nearest neighbor had a higher binding energy than He-divacancy with the second nearest neighbor in both screw and edge dislocation.

In cluster formation, interstitial He atoms always become cluster when distance between each interstitial He atoms are equal or less that the distance of nearest neighbor distance of Fe $\left(2.49 \mathrm{~A}^{0}\right)$ for screw dislocation. This happens whether interstitial He atoms closer or father away from the dislocation. The He interstitial atoms is easier to become cluster than substitution He atoms. Binding energy depends on the near neighbor atom and neighbor distance. In both edge and screw dislocations, the He-divacancy that near the dislocation relaxes closer to the dislocation. 


\section{Future}

Molecular dynamic simulation of $1 / 2 \mathrm{a}[111]$ screw dislocation in Ta was carried by Goddard II at al. [7]. They found that the equilibrium dislocation core has three-fold symmetry and spread out in three [112] direction on $\{110\}$ plane and the core energy per Burgers vector $\mathrm{b}$ is $1.36 \mathrm{eV} / \mathrm{b}$. As results, this symmetry will either induce or stop the migration of He atoms. Therefore, molecular statics, molecular dynamics and the dimer calculation of potential surface mapping can be used to study this effect. In addition, the rate of creation of He atoms in metals is substantially deteriorate mechanical properties, particular in iron at high temperature, $\mathrm{T} \geq 0.5 \mathrm{~T}_{\mathrm{m}}$, therefore, the molecular dynamics and the dimer calculation is a useful tools for study these effects for the present of $\mathrm{He}$ in alpha-iron. From these study, the migration of He along the dislocation, vacancy diffusion mechanism could be further study as well the diffusivity coefficient versus temperature and time.

\section{Acknowledgement}

This work was supported by the US Department of Energy, Office of Fusion Energy Science under contract DE-Aco6-76RLO 1830 and acknowledges participation in PNNL's Interfacial and Condensed Phase Summer Research Institute.

\section{References}

1. M.J. Caturla, T. Diaz de la Rubia, M. Fluss, J. Nucl. Mater. 323 (2003) 163-168.

2. H. Trinkaus, B. N. Singh, J. Nucl. Mater. 323 (2003) 229-242.

3. K. Morishita, R. Sugano, B. D. Wirth, J. Nucl. Mater. 323 (2003) 243-250.

4. G. J. Ackland, D. J. Bacon, A. F. Calder and T. Harry, Philos. Mag. A75, 713 (1997).

5. W. D. Wilson and R. d. Johnson: Interatomic Potential and Simulation of Lattice Defects, Plenum, 1972, p. 375.

6. D. E. Beck, Mol. Phys. 14, 311 (1968).

7. G. Wang, A. Strachan, T. Cagin, W. Goddard III, Mater. Sci. Engr. A309-310 (2001) 133-137. 


\title{
Creating site-specific nanoporous silicon surface by using Hydrogen Bubble
}

\author{
F. Ou \\ Department of Material Science and Engineering, \\ Rensselaer Polytechnic Institute, Troy, NY \\ and \\ L. V. Saraf, D.R. Baer \\ Pacific Northwest National Laboratory \\ Richland, WA
}

\begin{abstract}
Nanoporous silicon has been of major research interest since the discovery of its lightemission property in 1990. In addition, porous silicon has been shown to be a suitable chemical and biological material due to its large surface area. Surface functionality is especially important in these "wet" applications. In this work, we demonstrated the ability to use engineered defect to tune the formation of nanopores with specific pattern at specific site. This ability to form site-specific porous silicon is likely to become a powerful tool in the current wet application of porous silicon including the use as sensor arrays and microreactor.
\end{abstract}

In this study, we are using micro-fabrication techniques combined with reactivity of hydrogen bubbles to produce nanopores at well defined sites in order to create patterned nanoporous silicon surfaces. Hydrogen evolution through bubble formation from silicon surface during chemical etching is a known process. In an electro-chemical etching reaction, etch-current density and etch-time can be useful variables that may allow tuning or adjustment of related etching properties. Since etch-current density and time are directly related to rate of hydrogen $\left(\mathrm{H}_{2}\right)$ evolution and hydrogen bubble residence time on silicon surface, low current densities can be used to enhance the residence times. We use arrays of micro-fabricated pits on n-doped silicon surface to localize hydrogen bubbles at lower current densities $\sim 5 \mathrm{~mA} / \mathrm{cm}^{2}$. The reactivity of hydrogen bubbles is then used to produce nanopores in the vicinity of pits. We studied the effects of etch-time and current density on the nanostructure morphology by using scanning electron and optical microscopy. 


\title{
Research Summary: Large molecule characterization via FT-ICR
}

\author{
Jeffrey M. Spraggins II \\ Advisor: Dr. Murray Johnston \\ The University of Delaware \\ Department of Chemistry and Biochemistry
}

\begin{abstract}
Learning the experimental procedures and data interpretation of Fourier transform ion cyclotron resonance (FT-ICR) mass spectroscopy was the central theme to the research that was done during my stay at EMSL. The instrument used was an ESI-FTICR mass spectrometer with SID capabilities designed and built by Dr. Barlow and Dr. Laskin. The instrument utilizes a 6-T actively shielded superconducting magnet. The source region is composed of a collisional quadruplole that is operated in the RF mode only, a resolving quadrupole used for mass selection, and an accumulation quadrupole that is used for trapping resolved ions. The electrostatic ion guide, which consists of five ion lenses and a quadrupole bender, not only focuses the ion beam, but it is used to reduce the footprint of the instrument and eliminates the flow of neutral molecules from the source to the UHV region. The SID (surface induced dissociation) surface is introduced to the rear trapping plate of the cylindrical ICR cell using a home-built probe and vacuum-lock system. The details of the instrument have been published previously.1 the research focused on applications of FT-ICR involving large molecule characterization with an emphasis on protein and peptide analysis. The succeeding projects involve the characterization of samples of current interest to our research group.
\end{abstract}

\section{Oligomer Analysis}

Significance: The physiscochemical properties of organic molecules in secondary organic aerosol (SOA) formation is of great importance on the impact of SOA on the environment in terms of visibility, climate and health effects. Volatile organic species can be released into the atmosphere via plants or man made emissions. Recent studies have provided direct evidence for the production of high molecular weight compounds as a driving force for SOA production.2

Results: Samples were obtained by collecting aerosols by filtration and extraction using methanol. Standard samples were taken from a smog chamber in which toluene was the primary component and ambient samples were air samples taken outside of LDL. The methanol solutions were analyzed using ESI-FT-ICR to obtain high mass accuracy data to determine the molecular formula of the analyte and MS/MS via SID was used to identify the species. The spectrum on the left (Figure 1) shows the distribution of ions present in the standard sample while the spectrum on the right (Figure 2) is an example of MS/MS providing structural information on the $341 \mathrm{~m} / \mathrm{z}$ parent ion. Data is currently being processed. 


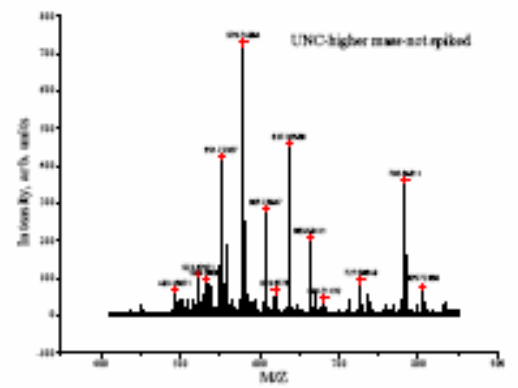

Figure 1: Distribution of species present in the standard sample.

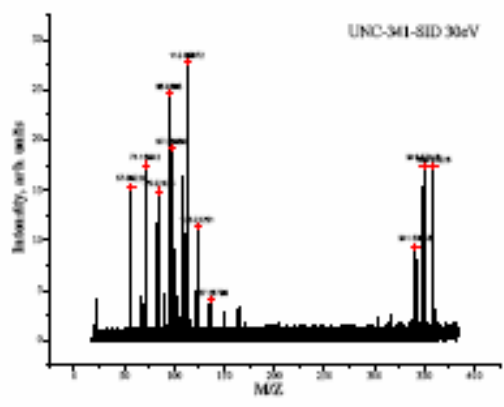

Figure 2: $30 \mathrm{eV}$ SID spectrum of the 341 $\mathrm{m} / \mathrm{z}$ parent ion of the standard sample.

\section{ZnS Clusters}

Significance: The study of $\mathrm{ZnS}$ clusters is of interest to both the environmental and material science communities. In terms of environmental studies, the understanding of the geochemical behavior and the bioavailability of trace metals is crucial to understanding the conditions of our natural water supplies. Metal sulfides ( $\mathrm{CuS}, \mathrm{FeS}$, and $\mathrm{ZnS}$ ) have been shown to make up over $90 \%$ of the dissolved sulfide concentrations in river waters. $3 \mathrm{ZnS}$ clusters are of interest to material scientists in terms of their applicability to quantum dots and high surface area catalysts.

Results: $\mathrm{ZnS}$ clusters were formed via the reaction of $\mathrm{Zn}(\mathrm{NO} 3) 2$ and Na2S under N2 pressure and analysis was done using ESI-FT-ICR. Results showed the definite formation of clusters containing both $\mathrm{Zn}$ and $\mathrm{S}$ as highlighted by the observed isotopic distributions (Figures 3) in relation to theoretical isotopic distributions (Figure 5) and the number of species at each nominal mass (Figure 4) in relation to theoretical masses (Table 1). The observed masses, however, do not match theoretical values and suggest the possibility of the addition of organics, biomolecules, methanol clusters or polymeric hydrogen. Further research is required to determine the cause of the observed mass defects. 


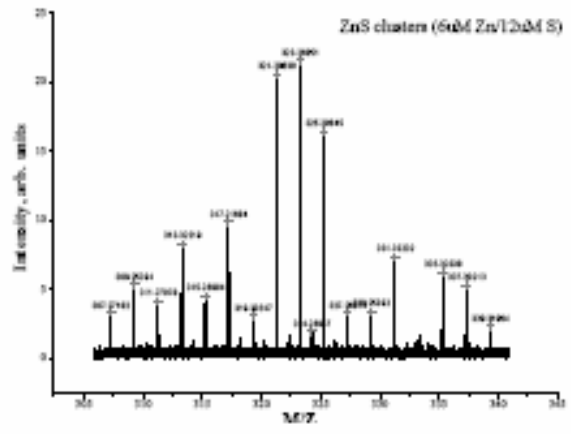

Figure 3: Distribution of clusters present

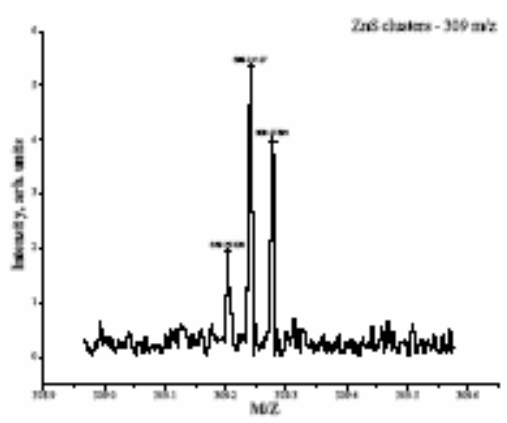

Figure 5: Peaks present at nominal mass $309 \mathrm{~m} / \mathrm{z}$

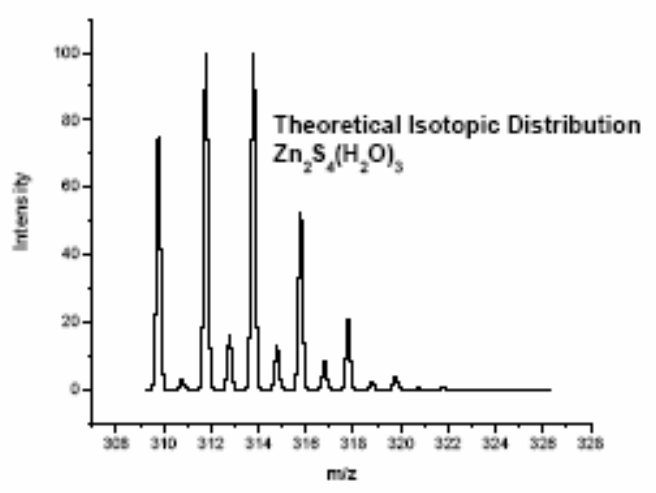

Figure 4: Theoretical isotopic distribution for $\mathrm{Zn}_{2} \mathrm{~S}_{4}\left(\mathrm{H}_{2} \mathrm{O}\right)_{3}$

Table 1: Theoretical ZnS Clusters in complex with three water molecules.

\begin{tabular}{|r|r|r|r|r|r|r|}
\hline & 1 & 2 & 3 & 4 & $\mathbf{Z n}$ \\
\hline 1 & 149.9329 & 213.8621 & 277.7912 & 341.7204 & \\
\hline 2 & 181.905 & 245.8341 & 309.7633 & 373.6924 & \\
\hline 3 & 213.877 & 277.8062 & 341.7353 & 405.6645 & \\
\hline 4 & 245.8491 & 309.7783 & 373.7074 & 437.6366 & \\
\hline 5 & 277.8212 & 341.7503 & 405.6795 & 469.6086 & \\
\hline 7 & 309.7933 & 373.7224 & 437.6516 & 501.5807 & \\
\hline 8 & 341.7653 & 405.6945 & 469.6236 & 533.5528 & \\
\hline 9 & 405.7374 & 437.6665 & 501.5957 & 565.5248 & \\
\hline 10 & 437.6815 & 469.6386 & 533.5678 & 597.4969 & \\
\hline $\mathbf{S}$ & & 501.6107 & 565.5396 & 629.469 & \\
\hline & & & & & $3 \mathrm{H} 2 \mathrm{O}$ \\
\hline
\end{tabular}




\section{Ozonated Peptides}

Significance: The analysis of ozonated peptides (angiotensin II and adrenocorticotropic hormone fragment 18-39) using ESI-FT-ICR is presented. The interaction of amino acids, peptides, and proteins with ozone has been studied in the past using spectrophotometric methods, and recently ozonolysis of free amino acids have been observed using ESI-MS and MS/MS techniques. Kinetic and mechanistic research efforts have shown the most reactive residues to be Met, Trp, Cys, His, and Phe.4 Additionally, studies have shown that the reactivity of individual amino acids is in the order Met $>\operatorname{Trp}>\operatorname{Tyr}>$ His while for the same residues, when part of a small peptide (2-4 amino acids), the reactivity is Met $>$ His $>\operatorname{Trp}>$ Tyr.5 This difference suggests that ozonation of larger peptides (8-10 residues) may also occur differently than free amino acids. The study of ozonated peptides has the potential to provide insight into the negative health effects of prolonged high levels of ozone exposure has on humans.

Experimental: Samples were prepared by allowing the peptides to react under flowing ozone for time periods up to 60 minutes using the apparatus seen here. Each peptide was purchased from Sigma-Aldrich and ozone was produced using a commercial ozone generator model PZ5 (Prozone International, Inc.). Analysis was done using ESI-FTICR. Oxygen Adduct Identification: The first aspect of this study was to obtain mass accuracy data of the parent ions and SID spectra at multiple collision energies. The objective was to show that the products due to ozonolysis are a result of oxygen addition and to use MS/MS to determine the location of the added oxygen atoms. Mass accuracy was able to determine that four oxygen adducts were produced due to the addition of 1,3 , 4 , and 5 oxygen atoms (Figure 6) and SID spectra suggest that addition takes place at the Tyr and His residues (Figures $7 \& 8$ ).

Structure Determination: Differences in fragmentation with addition of oxygen due to ozonolysis was also studied. The idea is to use energy-resolved surface-induced dissociation studies to determine fragmentation energetics as described in work done by Laskin, Bailey and Futrell.6 Figure 9 shows the loss of parent ion with increase of dissociation energy . Data is currently being analyzed, but initial results suggest ozonide formation on the His residue.

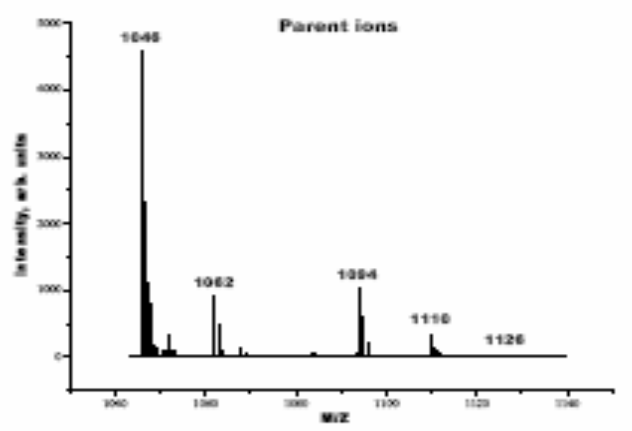

Figure 6: Spectrum of angiotensin II and four adducts due to the addition of oxygen

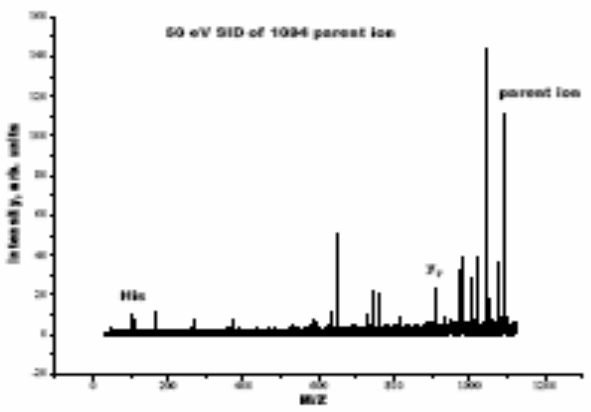

Figure 7: $50 \mathrm{eV}$ SID of 1094 parent ion 


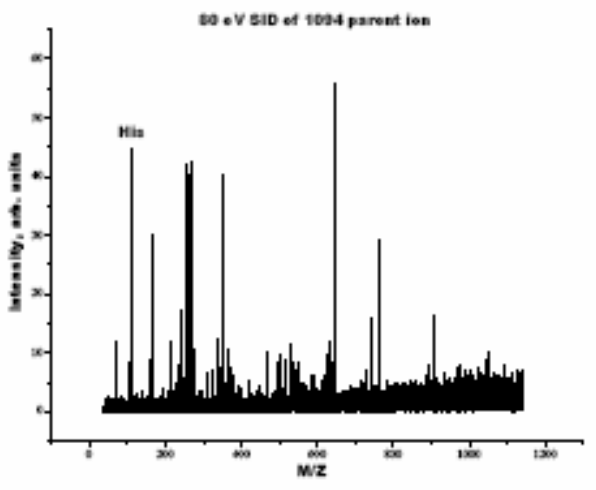

Figure 8: $80 \mathrm{eV}$ SID of 1094 parent ion

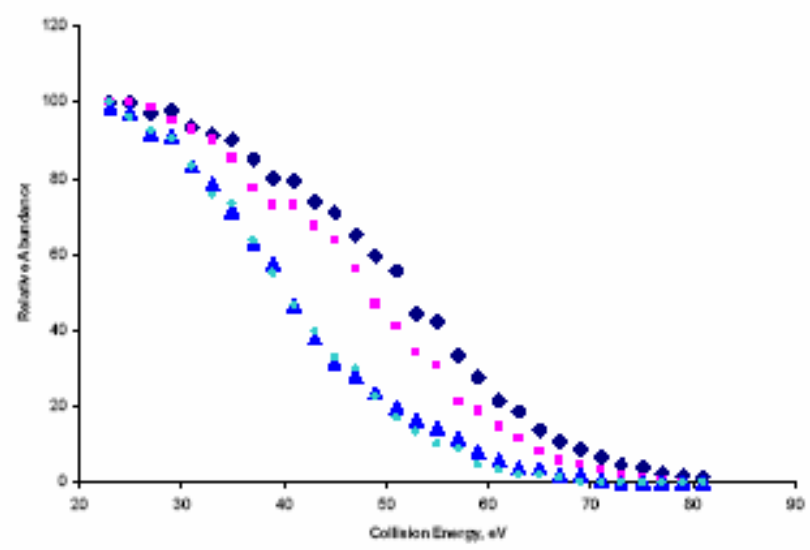

Figure 9: Plot showing the loss of parent ion with increasing collision energy in terms of number of oxygen atoms added. 


\section{References:}

(1) Laskin Julia; Denisov Eduard V; Shukla Anil K; Barlow Stephan E; Futrell Jean H Surface-induced dissociation in a Fourier transform ion cyclotron resonance mass spectrometer: instrument design and evaluation. Analytical chemistry (2002 Jul 15), 74(14), 3255-61.

(2) Tolocka, Michael P.; Jang, Myoseon; Ginter, Joy M.; Cox, Frederick J.; Kamens, Richard M.; Johnston, Murray V. Formation of Oligomers in Secondary Organic Aerosol. Environmental Science and Technology (2004), 38(5), 1428-1434.

(3) Rozan, Tim F.; Lassman, Michael E.; Ridge, Douglas P.; Luther, George W., III. Evidence for iron, copper and zinc complexation as multinuclear sulfide clusters in oxic rivers. Nature (London) (2000), 406(6798), 879-882.

(4) Pryor, W. A. G., D.H.; Church, D.F. J. Am. Chem. Soc. 1984, 106, 7094-7100.

(5) Kotiaho, T.; Eberlin, M.N.; Vainiotalo, P.; Kostiainen, R. J. Am. Soc. Mass Spectrom. 2000, 11, 526-535.

(6) Laskin, Julia; Bailey, Thomas H.; Futrell, Jean H. Fragmentation energetics for angiotensin II and its analogs from time- and energy-resolved surface-induced dissociation studies. International Journal of Mass Spectrometry (2004), 234(1-3), 89-99. 


\title{
n-Alkanes on $\mathrm{MgO}(100)$ I: |Coverage-dependent Desorption Kinetics of n-Butane
}

\author{
Steven L. Tait, \\ Department of Physics, Box 351560 \\ University of Washington, Seattle, WA 98195-1700 \\ Charles T. Campbell \\ Department of Chemistry, Box 351700, \\ University of Washington, Seattle, WA 98195-1700 \\ Zdenek Dohnálek, Bruce D. Kay", \\ Chemical Sciences Division Fundamental Sciences Directorate, \\ Pacific Northwest National Laboratory, Richland, WA 99352 \\ * Corresponding author. Telephone: (509)376-0028. Fax: (509)376-6066. \\ E-mail: bruce.kay@pnl.gov.
}

\begin{abstract}
$\underline{\text { Abstract }}$
High quality temperature programmed desorption (TPD) measurements of nbutane from $\mathrm{MgO}(100)$ have been made for a large number of initial butane coverages ( 0 $3.70 \mathrm{ML})$ and a wide range of heating ramp rates $(0.3-10 \mathrm{~K} / \mathrm{s})$. We present a TPD analysis technique which allows the coverage-dependent desorption energy to be accurately determined by mathematical inversion of a TPD spectrum, assuming only that the prefactor is coverage-independent. A variational method is used to determine the prefactor that minimizes the difference between a set of simulated TPD spectra and corresponding experimental data. The best fit for butane desorption from $\mathrm{MgO}$ is obtained with a prefactor of $10^{15.7 \pm 1.6} \mathrm{~s}^{-1}$. The desorption energy is $34.9 \pm 3.4 \mathrm{~kJ} / \mathrm{mol}$ at 0.5 ML coverage, and varies with coverage approximately as $E_{d}(\theta)=34.5+0.566 \theta+8.37 \exp (-\theta / 0.101)$. Simulations based on these results can accurately reproduce TPD experiments for submonolayer initial coverages over a wide range of heating ramp rates $(0.3-10 \mathrm{~K} / \mathrm{s})$. Advantages and limitations of this method are discussed.
\end{abstract}

\section{Introduction}

Alkane adsorption on oxide surfaces is of significant interest because of the wide use of oxides in heterogeneous catalysts as support materials and as active catalysts. For example, $\mathrm{MgO}$ catalysts are used for the oxidative coupling of methane to form $\mathrm{C}_{2}$ hydrocarbons or for methane oxidation to produce formaldehyde. ${ }^{1,2}$ The $\mathrm{MgO}(100)$ surface is one of the most thoroughly studied of all single crystal oxide surfaces from both experimental and theoretical approaches, ${ }^{3,4}$ and has also received significant attention as a support in model catalyst studies. ${ }^{5-7}$ Therefore the adsorption and desorption kinetics of alkane molecules on $\mathrm{MgO}$ and other oxide surfaces is of fundamental interest. Previous studies addressed the desorption of butane from metal surfaces such as $\mathrm{Cu}(111),{ }^{8} \mathrm{Pt}(111),{ }^{8-10} \mathrm{Ru}(001),{ }^{11}$ and $\mathrm{Au}(111) .{ }^{12}$ Few studies have been 
conducted on oxide surfaces; we note that Slayton et al. ${ }^{13}$ studied desorption of butane, hexane, and octane on aluminum oxide. Using Redhead peak temperature analysis and the variation of heating rate method, they obtained a desorption activation energy for butane from $\mathrm{Al}_{2} \mathrm{O}_{3}(0001)$ of $35.1 \pm 5.9 \mathrm{~kJ} / \mathrm{mol}$ with a prefactor of $4 \times 10^{10 \pm 2.5} \mathrm{~s}^{-1}$. Their data suggest that the relative magnitude of molecule-surface interactions compared to molecule-molecule interactions decreases with increasing alkane chain length. ${ }^{13}$

The desorption kinetics can yield significant information about the energy barrier to desorption and pre-exponential factor (prefactor) in the desorption rate equation. Recent studies of larger alkane molecules $\left(\mathrm{C}_{\mathrm{x}} \mathrm{H}_{2 \mathrm{x}+2}, \mathrm{x}=5-60\right)$ have elucidated their desorption kinetics on graphite. ${ }^{14-16}$ These results show a non-linear dependence of desorption energy on chain length due to partial detachment conformational entropy. ${ }^{14}$ This should be a negligible effect for the small alkane molecules and therefore a careful study is needed to form an accurate model of their interaction with the substrate. Experiments for small alkanes $(\mathrm{x}<5)$ have been elusive because of the low sample temperatures required for their adsorption.

This paper is the first in a two-article series regarding the desorption of small alkanes from $\mathrm{MgO}(100)$. In the present paper, we discuss the desorption kinetics of butane from $\mathrm{MgO}(100)$. We introduce a method to obtain coverage-dependent kinetic parameters from TPD experiments. We assume a coverage-independent prefactor and mathematically invert a TPD spectrum to obtain a coverage-dependent desorption energy. These data are used to simulate a set of TPD spectra with varying initial coverages. The simulated TPD set is compared to corresponding experimental data and the residual error is used as a measure of the quality of the kinetic parameters. The prefactor is treated as a variational parameter to minimize the difference between the experimental and simulated TPD. The present paper explains this method in detail and validates it so that it can be used as a general method for TPD analysis. This paper will also address the interactions of butane with $\mathrm{MgO}(100)$ and the coverage dependence of its desorption activation energy, thereby motivating an analysis which captures the coverage-dependence of the desorption kinetics.

In the accompanying paper, ${ }^{17}$ we use the analysis method presented here to study the desorption kinetics for a series of n-alkanes $\left(\mathrm{C}_{x} \mathrm{H}_{2 \mathrm{x}+2}, \mathrm{n}=1-10\right)$. We find that the desorption energy increases linearly with chain length, with a small y-intercept value. We also find that the prefactor increases by six orders of magnitude with increasing chain length, consistent with the increase in rotational entropy of the 2D-gas transition state.

\section{Experimental}

The experimental apparatus has been described in detail elsewhere. ${ }^{18}$ All experiments were conducted in an ultra-high vacuum chamber with a base pressure of $\sim 1 \times 10^{-10}$ Torr. High quality $\mathrm{MgO}(100)$ films were produced by vapor deposition of $\mathrm{Mg}$ from a directional source in a background of $1 \times 10^{-6}$ Torr $\mathrm{O}_{2}$ onto a $\mathrm{Mo}(100)$ substrate held at $600 \mathrm{~K}$. The sample is then annealed at $1000 \mathrm{~K}$ in the $\mathrm{O}_{2}$ background. For film thicknesses greater than 30 layers, as employed in the present work, a sharp low-energy electron diffraction (LEED) pattern is observed and temperature-programmed desorption (TPD) experiments demonstrate surface defect densities comparable to those obtained by UHV-cleavage of MgO crystals. ${ }^{18}$ The low electrical and thermal conductivity of 
magnesium oxide makes it advantageous to use a thin film of $\mathrm{MgO}$ rather than a cleaved bulk crystal.

The sample temperature can be controlled accurately from $20-2000 \mathrm{~K}$ by $\mathrm{He}$ cryostat cooling and resistive heating. The sample temperature is measured by a thermocouple spot-welded to the back of the sample. Calibration of the absolute temperature of the sample is done by multilayer desorption of $\mathrm{N}_{2}$ and $\mathrm{CH}_{4}$ at a moderate heating rate $(0.6 \mathrm{~K} / \mathrm{s}) .{ }^{19}$ The multilayer desorption peak position for about $2 \mathrm{ML}$ initial coverage for each molecule is referenced to previous measurements. This was simply a zero-offset correction for this sample and thermocouple mounting, assuming the proper slope of thermocouple voltage vs. temperature, which had been calibrated previously for this temperature control system.

A supersonic molecular beam was used to deposit n-butane, $\mathrm{C}_{4} \mathrm{H}_{10}$, at a rate of $0.13 \mathrm{ML} / \mathrm{s}$ at normal incidence to the sample. The beam nozzle was held at room temperature. During deposition, the sample was held at $24 \mathrm{~K}$, a temperature well below the onset of the butane multilayer desorption (see Figure 2). There was no dissociation of the butane on the $\mathrm{MgO}$ surface as confirmed by Auger electron spectroscopy (AES) and by the excellent reproducibility of subsequent TPDs upon repeated doses. The reflected flux of the molecular beam was measured by a quadrupole mass spectrometer (QMS) in a line-of-sight position, as a qualitative measure of the butane uptake by the sample and as a verification that molecular beam fluxes were the same from experiment to experiment.

TPD experiments were conducted by heating the sample at a constant rate and measuring the desorption rate of butane by QMS $(\mathrm{m} / \mathrm{e}=43)$ in a line-of-sight position at an acquisition rate of $22 \mathrm{~Hz}$. The quantitative analysis of desorption rates here assumes that the angular distribution of desorbing alkanes is independent of coverage and temperature. This was verified with methane by measuring TPD using mass spectrometers in both line-of-sight and background positions. Any errors associated with this assumption were minimized by locating the QMS at about 30 degrees from sample normal, near the so-called magic angle $(\sim 35$ degrees $) .{ }^{20}$ TPD measurements were made at a heating rate of $0.6 \mathrm{~K} / \mathrm{s}$ for 29 initial coverages of butane ranging from 0-3.70 ML. Every fourth experiment was conducted with the same dose time (10 s dose, 1.13 ML) to check that the deposition rate from the molecular beam was constant and to watch for slight drift in the temperature measurement. The deposition rate varied by no more that 3 $\%$ and showed no systematic drift. By comparing the desorption peak leading edges of this control set we concluded that the calibration of the temperature measurement varied by less than $0.25 \mathrm{~K}$ with no systematic trend.

Effect of temperature gradient on TPD line shape

We also made TPD measurements at 11 other temperature ramp rates in the range $0.05-10 \mathrm{~K} / \mathrm{s}$, each at seven initial coverages in the range $0.10-2.49 \mathrm{ML}$. We found the TPD signal-to-noise ratio at the lowest heating rates $(0.05-0.1 \mathrm{~K} / \mathrm{s})$ unacceptable for our detailed analysis. Although the absolute temperature of the sample had been calibrated as described above (at $0.6 \mathrm{~K} / \mathrm{s}$ ), it was found that a further additive correction to the thermocouple temperature was needed for heating rates of $1.0 \mathrm{~K} / \mathrm{s}$ and higher in order for the theromocouple to read the average sample temperature in each case. The thermocouple temperature lagged behind the average sample temperature by 0.3 to $4.5 \mathrm{~K}$ at heating rates of 1.0 to $10 \mathrm{~K} / \mathrm{s}$ due to the fast heating and finite thermal conductivity of 
the sample. This calibration was determined by monitoring the leading edges of the desorption peaks of the butane multilayer at several heating rates.

In Figure 1(a) we have plotted butane multilayer desorption peaks for $2.49 \mathrm{ML}$ initial coverage on a log scale. The leading edges of these peaks, acquired at various heating ramp rates, do not coincide as they should. As we discuss below, the desorption rate is a single-valued function of coverage and temperature. The leading edges of the TPD peaks for these various heating rates represent the limit where negligible desorption has occurred (each experiment has same initial butane coverage) and so we expect the peaks to be aligned in that region. As we will discuss below, the line shape of this multilayer desorption peak is characteristic of coverage-independent (zero-order) desorption kinetics in which case the leading edge should line up until the peak maximum. We observe that for the larger ramp rates $(1.0-10.0 \mathrm{~K} / \mathrm{s})$ there is a noticeable temperature shift in the data due to temperature gradients on the sample. In Fig. 1(b), the temperature axis was shifted to align this edge with that observed for a heating rates of $0.6 \mathrm{~K} / \mathrm{s}$, at which rate we had done the absolute temperature calibration described above. The applied shifts were $0.3,1.7,2.6,3.4$, and $4.5 \mathrm{~K}$ for heating rates of 1.0, 3.0, 5.0, 7.0, and $10.0 \mathrm{~K} / \mathrm{s}$, respectively. After correcting the temperature in this way, the leading edges of the multilayer desorption peaks line up to within $0.5 \mathrm{~K}$ at a desorption rate of 0.002 $\mathrm{ML} / \mathrm{s}$ and the positions of the peak maxima increase monotonically with increasing heating ramp rate.

Figure 1(c) is a log plot of the first-layer desorption peaks (at the same set of heating rates) for a submonolayer coverage $(0.42 \mathrm{ML})$ of butane. Without the above mentioned correction, the leading edges of these peaks for the various heating ramp rates do not coincide. Figure 1(d) shows these data corrected using the same temperature corrections applied to the multilayer peak in Fig. 1(b). We see that this correction aligns the leading edges of the first-layer peak in the limit where the coverage on the surface is approximately equal to the initial coverage (each curve has the same initial coverage). We note that the dynamic range of these data is three to four orders of magnitude above the background noise, making resolution of the leading edges in the limit $\theta \approx \theta_{0}$ possible.

Alignment of the leading edges of the multilayer peaks rather than the first-layer peaks was used to determine the temperature corrections needed for each heating rate because the first-layer peak leading edges may also be affected by intermolecular interactions which would prevent the adsorbed molecules from maintaining a dynamic equilibrium on the surface (i.e. inhomogeneous surface coverage of adsorbates). This effect was proven to be negligible in the present case, as the leading edges of the firstlayer peaks coincide to within $0.5 \mathrm{~K}$ after applying the temperature correction derived from the multilayer peaks [Fig. 1(d)].

This heating rate-dependent temperature correction is due to a temperature gradient which will exist on any sample of finite thermal conductivity with non-uniform heat input. In this case, heat transfer to the disk shaped sample occurs at the two contacts to the heating wires located on opposite edges of the back of the sample. The temperature is read at another point near the perimeter of the sample, but away from the heat inputs. The gradient across the sampled area depends on a number of factors: (a) the thermal conductivity of the sample, (b) the relative locations of the heat input to the sample and the thermocouple, and (c) the heating ramp rate. The temperature gradient leads to a convolved TPD peak due to different desorption rates from various regions on the sample 
which are at different temperatures. We have modeled the effect of temperature gradient on TPD using a simple finite element calculation based on the geometry of our sample. At heating rates in the range $1.0-10.0 \mathrm{~K} / \mathrm{s}$, the peak shape does not change significantly but there is a shift in the apparent TPD peak position, due to the difference between the average sample temperature and the temperature measured by the thermocouple. The effect at higher heating rates is a significant distortion of the TPD peak and so we did not conduct experiments at heating rates greater than $10 \mathrm{~K} / \mathrm{s}$.

The temperature shift required to align the leading edges of the multilayer peaks in Fig. 1(b) increases linearly with heating rate and is fit well by a line with a y-intercept of zero and a slope of $0.49 \mathrm{~s}$. A calculation done by Carter and Anton shows that a characteristic time for temperature gradients on a disk-shaped sample of radius $r$ is well estimated by calculating these gradients in a one-dimensional $\operatorname{rod}^{21}$ of half-length $l=r$. The thermal conductivity $\kappa$, density $\rho$, and specific heat $c_{p}$ of the sample material $(1.38$ $\mathrm{W} / \mathrm{cm} \cdot \mathrm{K}, 10.2 \mathrm{~g} / \mathrm{cm}^{3}$, and $0.251 \mathrm{~J} / \mathrm{g} \cdot \mathrm{K}$, respectively for $\left.\mathrm{Mo}\right)^{22}$ are used to calculate the thermal diffusivity of the substrate. The characteristic time can be calculated as:

$$
t_{c}=\frac{l^{2}}{\alpha}=\frac{l^{2} \rho c_{p}}{\kappa} .
$$

A detailed analysis of the thermal conductivity of our sample is beyond the scope of this paper, however, using Carter's model with the thermal properties of molybdenum, we estimate the time constant to be on the order of $t_{c}=0.5 \mathrm{~s}$ for a molybdenum rod with halflength $l=5 \mathrm{~mm}$, corresponding to the radius of our disk sample. This time constant can be used to estimate the magnitude of the temperature gradient in the sample where it is assumed that the two ends of the rod are at the same temperature. The gradients predicted by Carter's model ${ }^{21}$ are of magnitude $\Delta T=\beta t_{c} / 2$. The gradients on our sample are estimated to be 0.075 to $2.5 \mathrm{~K}$ for ramp rates of $0.3-10 \mathrm{~K} / \mathrm{s}$, comparable to the temperature shifts we observe (within the factor of order one that Carter quotes for the accuracy of his model). ${ }^{21}$

We will discuss below our method for analyzing the TPD results as well as two well-known methods, the Redhead peak temperature analysis and the complete analysis. We note here that the temperature correction described above is critical for each of these analysis techniques to provide reliable results. We do not elaborate on this point as it has been discussed previously regarding the complete analysis technique. ${ }^{21}$ The data presented and analyzed in the following discussion have all been temperature corrected according to the procedure outlined in this section.

\section{$\underline{\text { Results and Discussion }}$}

\section{TPD with varying initial coverage}

The TPD data for a series of initial coverages of butane on $\mathrm{MgO}(100)$ are plotted in Figure 2. The 16 initial coverages of butane are in the range 0-1.76 ML. Thirteen other initial coverages from $0.24-3.70 \mathrm{ML}$ were also studied, but are not shown for clarity of presentation. There are two prominent, well-resolved desorption peaks for butane: desorption from the multilayer $(99 \mathrm{~K})$ and desorption from the first layer $(110 \mathrm{~K})$ of butane.

The butane multilayer desorption peak has a shape characteristic of zero-order (coverage-independent) desorption kinetics, where the leading edges for various initial 
coverages line up until their maxima, at which the multilayer is exhausted and the desorption rate drops sharply. With increasing initial coverage the peak maximum increases in intensity and shifts to higher temperature. For a 2.0 ML initial coverage and a ramp rate of $0.6 \mathrm{~K} / \mathrm{s}$ the peak maximum is positioned at approximately $99 \mathrm{~K}$. The second and higher layers were not resolved in this multilayer desorption peak. The desorption peak from the first layer of butane on the surface is centered at $110 \mathrm{~K}$. It is characteristic of desorption kinetics that are first-order in butane coverage (i.e., nearly the same peak position for any initial coverage). There is a slight $(2 \mathrm{~K})$ shift of the first-layer peak to higher temperature with increasing coverage in the range 0.4-1.0 ML, indicating an attractive interaction between adsorbed butane molecules. One monolayer (ML) is defined to be the number of molecules per unit area required to saturate the first-layer peak. The initial coverage was determined for each spectrum by integration of the desorption rate with respect to time and normalizing the integrated area to the area of the saturated first-layer peak.

Also noticeable on this first-layer peak is a high-temperature tail. It is clear from the coverage evolution of Figure 2 that this tail is not due to a limitation in pumping speed ( $>1000 \mathrm{~L} / \mathrm{s}$ in this chamber), as the tail saturates in height at relatively low initial coverage and does not increase in size proportional to the first-layer peak. At the lowest initial coverages shown in Figure 2 it becomes apparent that this tail is a smaller desorption peak which we interpret to be due to desorption of butane from defect sites on the surface. The saturation of this peak before the filling of the first-layer peak indicates that the butane molecules have sufficient mobility on the $\mathrm{MgO}(100)$ surface to find the most energetically favorable adsorption site. The area of this "defect" peak is difficult to deconvolute from the peak at $110 \mathrm{~K}$, but it appears to have saturated at an initial coverage of $\sim 0.2 \mathrm{ML}$ in Fig. 2. We compare this to previous desorption studies of $\mathrm{D}_{2} \mathrm{O}^{23}$ and $\mathrm{CO}^{24}$ from single-crystal $\mathrm{MgO}(100)$ surfaces which show a similar high temperature tail. We estimate that the area of the tail in those studies corresponds to approximately $0.15 \mathrm{ML}$ of adsorbate. It has been remarked previously that the amount of the adsorbate that is influenced by defect sites cannot be equated to the density of surface defects, as the binding of an adsorbate molecule in the vicinity of a defect-bound adsorbate molecule may also be influenced by the defect or several adsorbate molecules could be stabilized by one defect. ${ }^{18}$

The presence of these defect sites (of which there may be several species with unique binding energies) as well as the shift in the first-layer peak due to intermolecular attraction make it clear that the effective desorption energy barrier must have some dependence on the coverage of butane. At low butane coverages it represents a convolution of the activation energies for desorption from the various defect sites with those for desorption from $\mathrm{MgO}$ terrace sites. The effect of this convolution on the desorption energy decreases with increasing coverage. Additionally, the energy shows some increase with coverage due to the adsorbate-adsorbate attraction. Therefore, an analysis of these data to yield coverage-dependent kinetic parameters is necessary to accurately characterize this system.

From the high-quality TPD data presented here we are able to extract the coverage-dependence of the desorption kinetics using an analysis which has been presented previously ${ }^{18}$ and which we will elaborate and develop further here. The coverage of butane, $\theta$, on the surface at any given temperature, $T$, is calculated by 
integrating the desorption rate signal with respect to temperature (time) from $T$ to the limit $T \rightarrow \infty$, i.e.

$$
\theta(T)=\frac{1}{\beta} \int_{T}^{\infty} \frac{d \theta}{d T^{\prime}} d T^{\prime}
$$

where $\lim _{T \rightarrow \infty} \theta(T)=0$ and $\beta=\frac{d T}{d t}$.

Each TPD spectrum provides the desorption rate $r(\theta, T)$ versus time for a given initial coverage and heating rate. The desorption rate is a single-valued function of temperature and coverage. The Polanyi-Wigner equation gives the desorption rate, $r$, as a function of $\theta$ and $T$ :

$$
r(\theta, T)=-\frac{d \theta}{d t}(\theta, T)=v_{n}(\theta, T) e^{-E_{d}(\theta) / R T} \theta^{n}
$$

where $\mathrm{R}$ is the universal gas constant, $n$ the order of desorption, $E_{d}$ the activation energy for desorption, and $v$ the pre-exponential factor. By mathematical inversion, the coverage-dependent desorption activation energy, $E_{d}(\theta)$, can be obtained from the Polanyi-Wigner equation:

$$
E_{d}(\theta)=-R T \ln \left[-\frac{d \theta / d t}{v \theta}\right]
$$

Here we assumed that desorption is a first-order process $(n=1)$ and that $v$ does not vary with coverage or temperature. The latter assumption is somewhat arbitrary as the coupling of $v(\theta, T)$ and $E(\theta)$ would allow the coverage dependence to be captured mathematically by either variable. Physically, a variation of the prefactor with coverage would occur if the partition function of the adsorbate varied significantly in different adsorption sites on the surface or due to adsorbate-adsorbate interactions. Energy will change with coverage as various adsorption sites (e.g. point defects, step defects, kink defects, terrace sites, etc.) will have different coordination numbers of the substrate ions to the adsorbate leading to different strengths of the physisorption interaction. Also it is clear that van der Waals forces (or any other interactions) between the adsorbates will lead to changes in the desorption activation energy with coverage. Therefore the coverage dependence of the energy term is generally expected and its interpretation in terms of energy variations due to various adsorption sites and adsorbate-adsorbate interactions is well established.

Figure 3 shows the coverage-dependent desorption energy of butane from the $\mathrm{MgO}(100)$ surface for each of five assumed values of the prefactor $\left(v=10^{14}, 10^{15}, 10^{16}\right.$, $10^{17}, 10^{18} \mathrm{~s}^{-1}$ ). Each energy curve in Fig. 3 was calculated using equation (4) and a TPD spectrum of 1.2 ML initial coverage of butane with a heating rate of $0.6 \mathrm{~K} / \mathrm{s}$. The general behavior (shape) of the coverage-dependent energy curve is the same for each choice of $v$. At low coverages, the energy is high, corresponding to the binding of butane molecules at the point and step defects on the oxide surface. The energy decreases as the coverage increases to about $0.3 \mathrm{ML}$ and the butane molecules begin to fill the $\mathrm{MgO}$ terraces. In the coverage range $0.3-0.8 \mathrm{ML}$ the desorption energy appears to be nearly constant. However, there is a gradual rise $(<1 \%)$ of the desorption energy with increasing coverage in this region due to attractive interactions between the molecules. This corresponds to the slight shift in the desorption peak toward higher temperature with increasing initial coverage. As the coverage increases above 1.0 ML, the desorption 
energy again decreases as the molecules compress the first layer and begin to fill the second layer. ${ }^{25}$ These characteristics of the desorption energy curve are independent of $v$. While the general shape of the curves are similar, it is clear that the absolute energy shifts to higher values of $E_{d}$ with increasing $v$.

For purposes of comparison, we show in Figure 4 experimental TPD data (circles) for four initial coverages $(0.42,0.58,0.84$, and $1.20 \mathrm{ML})$. The lines in Figure 4 are simulations of the experimental TPD data using the energy result shown in Figure 3. Each line style corresponds to a different assumed value for the prefactor, each varying by a factor of ten. The highest initial coverage data set shown in Figure 4 (1.2 ML) was used to obtain the desorption energy values in Fig. 3 for each of the five prefactors. The simulated TPD spectra for that initial coverage fit perfectly for any prefactor value, since these experimental data were mathematically inverted to yield the coverage-dependent desorption energy. For any choice of $v$, one can choose a desorption energy value that will yield a given desorption rate and therefore construct a function $E_{d}(\theta)$ which will perfectly simulate the same TPD data back again. Other constraints are necessary to determine an accurate value of the prefactor. We have developed a methodology which yields a reliable value for the prefactor (within the assumption that it does not vary with coverage) by using the calculated $E_{d}(\theta)$ to simulate a set of TPD spectra at different initial coverages.

We see that the quality of the fit for the three lower initial coverages in Fig. 4 depends strongly on the choice of prefactor. The correct combination of $v$ and $E_{d}(\theta)$ should be able to accurately reproduce TPDs for any set of initial coverages. Of the five prefactors used to generate the simulations in Figure 4, it appears that $10^{16} \mathrm{~s}^{-1}$ is the best choice. Simulations based on lower prefactors show less agreement with the experimental data as the desorption rate is too high on the leading edge of the peak then too low on the trailing edge of the peak, causing the desorption peak to be smaller in height and to be centered at a lower temperature compared to the experimental data. Higher choices of prefactor show the opposite trend: desorption rate is too low on the leading edge and too high on the trailing edge, causing the peak to be too tall and shifted to higher temperature. Thus, by treating the prefactor as a variational parameter to optimize the fit between experimental and simulated TPDs, we can find the prefactor that best matches the kinetics of the desorption process.

The quality of the fit of the simulation to the experimental data is quantified by calculating a $\chi^{2}$ error between simulated and experimental TPD curves for five initial coverages (not including the inverted experimental set), only three of which are shown in Figure 4 for clarity of presentation. The $\chi^{2}$ error is defined as the sum of the squares of the residuals of the simulation compared to the experiment at each data point on the experimental TPD spectrum. As shown in Figure 5 the magnitude of the $\chi^{2}$ error has a well-defined minimum with respect to the assumed value of prefactor. These error values have been fit with a polynomial function (solid line in Figure 5) and the minimum of that function is indicated by the open circle at $v=10^{15.7} \mathrm{~s}^{-1}$. The best value of $v$ from Fig. 5 is also very close to the best "fit by eye" one would obtain from Fig. 4. As seen in Fig. 4, an order of magnitude change in $v$ leads to noticeable decline of the quality of the fit, and therefore we estimate the error in $\log v$ to be less than $\pm 10 \%$. Using the best fit value for the prefactor, we calculate the coverage-dependent desorption energy of butane from 
$\mathrm{MgO}(100)$, which has a value of $34.9 \pm 3.4 \mathrm{~kJ} / \mathrm{mol}$ at $0.5 \mathrm{ML}$ coverage. The stated error here corresponds to the $10 \%$ uncertainty in the log of the prefactor.

This result, the "best fit" prefactor and corresponding coverage-dependent desorption energy, is used to simulate a set of TPDs for five initial coverages, shown as solid lines in Figure 6 . The solid points in Figure 6 are experimental data for initial coverages $0.19,0.42,0.58,0.84$, and $1.00 \mathrm{ML}$. The simulations capture the peak position (desorption temperature) of the experiment and they also reproduce very well the shape of the desorption curve, including the subtle shifts in temperature due to adsorbateadsorbate interactions as well as the high temperature shoulder due to the influence of the defect states on the desorption kinetics of butane.

To a good approximation, the coverage dependence of the desorption energy can be represented well over the coverage range $0-0.8 \mathrm{ML}$ by the analytic function:

$$
E_{d}(\theta)=E_{0}+\alpha \theta+E_{d e f} \exp \left(-\theta / \theta_{d e f}\right)=34.5+0.566 \theta+8.37 \exp (-\theta / 0.101),
$$

where all energies are in $\mathrm{kJ} / \mathrm{mol}$. This function has been constructed to express the general behavior of the desorption energy with respect to coverage without unnecessary complexity. This function is plotted in Figure 7 along with the result of the inversion analysis. We see that it matches the inversion result well and we note that using this analytic result to simulate TPD (not shown here) shows a very good fit to the experimental results. The first term $E_{0}$ represents the desorption energy one would obtain by extrapolating the linear region of the coverage-dependent energy curve back to the limit of zero coverage. This is the number that could most readily be interpreted as the activation energy for desorption of an isolated butane molecule from a $\mathrm{MgO}(100)$ terrace site (i.e. in the absence of defect sites and adsorbate - adsorbate interactions). The factor $\alpha$ is the increase in desorption energy per ML increase in coverage; the positive sign of this number indicates an attractive interaction between butane molecules on the $\mathrm{MgO}(100)$ surface. This interaction is weak, accounting for only $1.3 \%$ of the desorption activation energy at $0.8 \mathrm{ML}$ coverage. The strength of this interaction is smaller than the bulk sublimation energy by more than a factor of ten. This may be due to adsorbed butane molecules being separated by a distance that is far from their optimum bulk separation (either greater or less) due to their packing being determined to a much greater extent by their interaction with the $\mathrm{MgO}$ than by butane-butane interactions.

Alternatively, the factor $\alpha$ may be small because dipoles induced in the butane molecules by the $\mathrm{MgO}$ substrate may mitigate the inherent attraction between the molecules. $E_{\text {def }}$ is the energy difference between $E_{0}$ and the measured desorption energy at zero coverage, $E_{d}(0)$, and is related to the difference between the adsorption energy of a butane molecule adsorbed on an $\mathrm{MgO}$ terrace site compared to one adsorbed at a defect site. The factor $\theta_{d e f}$ corresponds to the rate at which the influence of defect sites on the energy result decays with increasing coverage. It is related to the density (coverage) of defect sites on the oxide surface as well as to the degree to which defect sites influence the adsorption of molecules that are near molecules adsorbed at defects. This term may give a more reliable insight into the actual density of defects on the surface rather than looking at the area under the defect shoulder in the TPD spectrum, but we do not wish to over-interpret this empirical fit function. 


\section{TPD with varying heating ramp rate}

Variation of the heating ramp rate over a set of TPD experiments allows one to extract more information about the desorption kinetics by recording the desorption rate for a given coverage at several different values of temperature. TPD experiments were conducted using a set of eleven different heating ramp rates $(0.05,0.07,0.1,0.3,0.5,0.7$, $1.0,3.0,5.0,7.0$, and $10.0 \mathrm{~K} / \mathrm{s})$ and seven initial coverages $(0.10,0.22,0.44,0.73,0.91$, 1.20 , and $2.49 \mathrm{ML})$.

Experimental data points for butane desorption ( $0.44 \mathrm{ML}$ initial coverage) from $\mathrm{MgO}$ at heating rates of $0.3,0.5,0.6,0.7,1.0,3.0,5.0,7.0$, and $10.0 \mathrm{~K} / \mathrm{s}$ are plotted in two panels in Figure 8 and have been temperature-corrected as discussed in the experimental section. The difference in the lower panel from the upper is only that the yaxis has been rescaled to show more detail in the desorption peak for the five lowest heating rates. We have also simulated TPD at these heating rates using the desorption energy vs. coverage curve discussed previously which was calculated by inversion of a TPD taken at $0.6 \mathrm{~K} / \mathrm{s}$ (highest peak in Figure 4), using the assumed prefactor value of $v=$ $10^{15.7} \mathrm{~s}^{-1}$. These simulations are plotted as solid lines in Figure 8 . The leading edges of the simulated curves line up perfectly as they are simulated without temperature gradients. We see that the agreement between simulation and experiment is excellent for the choice of $v=10^{15.7} \mathrm{~s}^{-1}$, demonstrating the robustness of our inversion method over a wide range of heating ramp rates. The simulation matches experiment best at ramp rates of $0.5-3.0$ $\mathrm{K} / \mathrm{s}$ (i.e. near the heating rate of the inverted spectrum, $0.6 \mathrm{~K} / \mathrm{s}$ ). In Figure 8 we see that at higher ramp rates the simulation misses the experimental data by about $0.5 \mathrm{~K}$, an error of less than $0.5 \%$ in temperature or desorption energy. The desorption peak shape is reproduced well by the simulation, even at high ramp rate. The high temperature tail due to desorption from defect states is matched well by the experiment and scales appropriately with heating rate.

Using the set of TPD data at various heating rates, the desorption energy and prefactor information can be extracted from the set of desorption peak temperatures, using the Redhead method. ${ }^{26-28}$ This method has been used in several previous studies of the desorption of alkanes, $\mathrm{C}_{\mathrm{x}} \mathrm{H}_{2 \mathrm{x}+2} .{ }^{8,11,13,15}$ In this method, the Polanyi-Wigner equation is evaluated at the desorption peak temperature and rearranged to make an Arrhenius form for the peak desorption temperature, $T_{p}$, at different heating rates, $\beta$ :

$$
\ln \left(\frac{\beta}{T_{p}^{2}}\right)=-\ln \left(\frac{E_{d}}{k v}\right)-\frac{E_{d}}{k} \frac{1}{T_{p}} .
$$

Implicit in this analysis is the assumption of coverage-independent desorption-kinetics parameters ( $v$ and $E_{d}$ ). We have compiled the peak temperatures $T_{p}$ for the TPD data shown in Figure 8 and we plot $\ln \left(\frac{\beta}{T_{p}^{2}}\right)$ Vs. 1/T in Figure 9. We see that these points fit well

to a line (shown in Fig. 9). The slope of the linear fit in Fig. 9 corresponds to a desorption energy of $36.6 \pm 0.7 \mathrm{~kJ} / \mathrm{mol}$. The $y$-intercept of that line corresponds to a prefactor value of $10^{16.7 \pm 0.3} \mathrm{~s}^{-1}$ (quoted errors correspond to statistical error in the linear regression). These results are in agreement with the results of the inversion analysis, $v=10^{15.7 \pm 1.6} \mathrm{~s}^{-1}$ and $E_{d}(\theta=0.5 \mathrm{ML})=34.9 \pm 3.4 \mathrm{~kJ} / \mathrm{mol}$, within the error of the two analysis methods. We can also compare the peak temperature analysis results to previous TPD studies of butane on 
$\mathrm{Al}_{2} \mathrm{O}_{3}(0001)$ analyzed using this Redhead peak temperature method. On that surface, a desorption energy of $35.1 \pm 5.9 \mathrm{~kJ} / \mathrm{mol}$ with a prefactor of $10^{10.6 \pm 2.5} \mathrm{~s}^{-1}$ was measured. $^{13}$

We have used the result from the simplified Redhead peak temperature analysis to simulate the TPD experiment, and have plotted these in Figure 10. The solid points represent the experimental data and dashed lines are simulations based on the Redhead analysis approach. For comparison, we also plot the simulations from our inversion analysis as solid lines. We see that the Redhead method matches the desorption peak temperature for the higher initial coverages well, but that its ideal first-order peak shape does not match the experimental peak shape. Also, this method completely misses the small peak (tail) due to desorption from defect states, since it assumes coverageindependent kinetics. The simulation based on the inversion analysis (solid lines) contains coverage-dependent parameters and hence matches the data very well.

A common analysis method which yields coverage-dependent values for both prefactor and desorption energy is referred to as complete analysis. ${ }^{29,30}$ This technique $^{3}$ consists of measuring TPD over a wide range of coverages and/or heating ramp rates to allow measurement of $\frac{d \theta}{d t}(T, \theta)$ for various combinations of $\theta$ and $T$. From these data, isosteric (constant coverage) Arrhenius lines of $\ln \left[\frac{d \theta}{d t}(T, \theta)\right]$ vs. 1/T are constructed and fit to lines. Then the desorption energy and prefactor can be calculated for discrete coverages corresponding to each isostere. ${ }^{21}$ A significant disadvantage to this complete analysis approach is that kinetic parameters are obtained only at discrete coverage values, which may make it difficult to accurately describe the desorption kinetics if these parameters vary rapidly in coverage, as in the present case. The inversion analysis that we have described here calculates a desorption energy value for each point on the TPD spectrum.

We have carried out this complete analysis on our data and obtained results that are generally in good agreement with the inversion analysis result. The TPD points lined up well for isosteres in the range 0.4 to $0.9 \mathrm{ML}$. In that range, the prefactor and desorption energy show little coverage dependence, varying by less than $2 \%$, and have values of $v=10^{16.4} \mathrm{~s}^{-1}$ and $E_{d}=36.4 \mathrm{~kJ} / \mathrm{mol}$. For coverages less than $0.4 \mathrm{ML}$, the isosteric plots of desorption rate from different initial coverage sets of TPD did not fall on the same line and so there is a large statistical uncertainty $( \pm 4-12 \%)$ in those results. The complete analysis provides coverage-dependent energy and prefactor information at discrete coverages in the range 0.4-0.9 ML. The inversion method presented above produces reliable coverage-dependent desorption energy results at continuous coverages over the full range submonolayer coverage range.

\section{Conclusion}

We have conducted temperature programmed desorption experiments to obtain coverage dependent kinetics parameters for desorption of n-butane from the $\mathrm{MgO}(100)$ surface. We invert the TPD data to obtain desorption energy vs. coverage information for an assumed value of the prefactor and show that the choice of prefactor strongly affects the quality of simulated TPD. By optimizing the match of the TPD simulation to data, we obtain a best fit prefactor and coverage-dependent desorption energy which can be used 
to accurately simulate TPD spectra over a wide range of initial coverages and heating rates. In the next article, we employ this methodology to study the chain length dependence of desorption kinetic parameters over a range of small alkane molecules. ${ }^{17}$

\section{Acknowledgements}

BDK and ZD funded by the U. S. Department of Energy Office of Basic Energy Sciences and Pacific Northwest National Laboratory (PNNL) LDRD funds. SLT supported by a graduate student fellowship award from the University of Washington/PNNL Joint Institute for Nanoscience and acknowledges participation in PNNL's Interfacial and Condensed Phase Summer Research Institute. PNNL is a multiprogram National Laboratory operated for the U. S. Department of Energy by Battelle Memorial Institute under contract DE-AC06-76RLO 1830. CTC acknowledges support by the U. S. Department of Energy, Office of Basic Energy Sciences, Chemical Sciences Division.

\section{References}

1 J. S. J. Hargreaves, G. J. Hutchings, R. W. Joyner, and C. J. Kiely, Journal of Catalysis 135, 576 (1992).

2 J. S. J. Hargreaves, G. J. Hutchings, and R. W. Joyner, Nature 348, 428 (1990).

3 H. J. Freund, H. Kuhlenbeck, and V. Staemmler, Reports on Progress in Physics 59, 283 (1996).

V. E. Henrich and P. A. Cox, The Surface Science of Metal Oxides (Cambridge University Press, Cambridge, 1994).

C. T. Campbell, Surface Science Reports 27, 1 (1997).

M. Baumer and H. J. Freund, Progress in Surface Science 61, 127 (1999).

C. R. Henry, Surface Science Reports 31, 235 (1998).

R. Z. Lei, A. J. Gellman, and B. E. Koel, Surf. Sci. 554, 125 (2004).

J. F. Weaver, A. F. Carlsson, and R. J. Madix, Surface Science Reports 50, 107 (2003).

M. Salmeron and G. A. Somorjai, Journal of Physical Chemistry 85, 3835 (1981).

J. L. Brand, M. V. Arena, A. A. Deckert, and S. M. George, Journal of Chemical Physics 92, 5136 (1990).

S. M. Wetterer, D. J. Lavrich, T. Cummings, S. L. Bernasek, and G. Scoles, Journal of Physical Chemistry B 102, 9266 (1998). 
R. M. Slayton, C. M. Aubuchon, T. L. Camis, A. R. Noble, and N. J. Tro, Journal of Physical Chemistry 99, 2151 (1995).

A. J. Gellman and K. R. Paserba, Journal of Physical Chemistry B 106, 13231 (2002).

K. R. Paserba and A. J. Gellman, Journal of Chemical Physics 115, 6737 (2001).

K. R. Paserba and A. J. Gellman, Physical Review Letters 86, 4338 (2001).

S. L. Tait, Z. Dohnalek, C. T. Campbell, and B. D. Kay, accompanying article (2004).

Z. Dohnalek, G. A. Kimmel, S. A. Joyce, P. Ayotte, R. S. Smith, and B. D. Kay, Journal of Physical Chemistry B 105, 3747 (2001).

H. Schlichting and D. Menzel, Review of Scientific Instruments 64, 2013 (1993).

S. W. Pauls and C. T. Campbell, Surf. Sci. 226, 250 (1990).

R. N. Carter and A. B. Anton, Journal of Vacuum Science \& Technology AVacuum Surfaces and Films 10, 344 (1992).

D. R. Lide, ed., CRC Handbook of Chemistry and Physics (CRC Press, Inc., Boca Raton, Florida, 1992).

M. J. Stirniman, C. Huang, R. S. Smith, S. A. Joyce, and B. D. Kay, J. Chem. Phys. 105, 1295 (1996).

R. Wichtendahl, M. Rodriguez-Rodrigo, U. Hartel, H. Kuhlenbeck, and H. J. Freund, Physica Status Solidi a-Applied Research 173, 93 (1999).

G. A. Kimmel, M. Persson, Z. Dohnalek, and B. D. Kay, J. Chem. Phys. 119, 6776 (2003).

P. A. Redhead, Vacuum 12, 203 (1962).

D. A. King, Surf. Sci. 47, 384 (1975).

J. L. Falconer and R. J. Madix, Surf. Sci. 48, 393 (1975).

A. M. de Jong and J. W. Niemantsverdriet, Surf. Sci. 233, 355 (1990).

J. L. Taylor and W. H. Weinberg, Surf. Sci. 78, 259 (1978). 


\title{
Thermal Oxidation Behavior of Polymer-Derived Si(Al)CN ceramics
}

\author{
Yiguang Wang and Linan An \\ Adv. Mats. Proc. And Analysis Center \\ University of Central Florida \\ Orlando, FL \\ And \\ Chongmin Wang and Sarah D. Burton \\ Pacific Northwest National Laboratory \\ Richland, WA
}

\begin{abstract}
The oxidation behaviors of polymer-derived amorphous Silicoaluminum carbonitride $\left[\mathrm{Si}(\mathrm{Al}) \mathrm{CN}\right.$ ] ceramics are investigated in the range of $900^{\circ} \mathrm{C}-1200^{\circ} \mathrm{C}$ in ambient air. Three kinds of ceramics were studied: $\mathrm{SiC}_{0.99} \mathrm{~N}_{0.85}(\mathrm{SiCN}), \mathrm{SiAl}_{0.02} \mathrm{C}_{0.99} \mathrm{~N}_{0.85}$ ( $\mathrm{SiCN}-5 \mathrm{Al})$, and $\mathrm{SiAl}_{0.04} \mathrm{C}_{0.83} \mathrm{~N}_{0.93}(\mathrm{SiCN}-10 \mathrm{Al})$. All of the specimens are fully dense. The oxide scale growth of SiCN ceramics in the whole temperature range can be expressed by a parabolic law. At $900^{\circ} \mathrm{C}$, the oxidation behavior of $\mathrm{Si}(\mathrm{Al}) \mathrm{CN}$ ceramics is similar to SiCN ceramics, showing parabolic growth. While at higher temperature, $\mathrm{Si}(\mathrm{Al}) \mathrm{CN}$ ceramics show two-stage oxidation behavior-- faster scale growth followed by a slower one. ${ }^{27} \mathrm{Al}$ MAS-NMR and ${ }^{28} \mathrm{Si}$ MAS-NMR indicate structural change during oxidation at different temperate and different oxidation time. The structural change of the oxide scale will account to the oxidation behavior.
\end{abstract}




\title{
Structural Evolution of Polymer-Derived Si(Al)CN Ceramics
}

\author{
Yiguang Wang and Linan An \\ Adv. Mats. Proc. And Analysis Center \\ University of Central Florida \\ Orlando, FL \\ And \\ Chongmin Wang and Sarah D. Burton \\ Pacific Northwest National Laboratory \\ Richland, WA
}

\begin{abstract}
The $\mathrm{Si}(\mathrm{Al}) \mathrm{CN}$ ceramics were prepared by pyrolysis of aluminum isopropoxide modified Polyurea(methylvinyl)silazanes $\left(\right.$ Ceraset $\left.^{\mathrm{TM}}\right)$. The structural evolution was studied by FT-IR, NMR, XRD, and TEM. The results showed that the aluminum greatly influence the structural change. The combination of aluminum in the structure retards the crystallization of these ceramics and affects the final crystallization phases.

In this summer, I worked with Chongmin Wang and Sarah Burton in PNL. With Chongmin, I worked on TEM to study the structural evolution of polymer-derived $\mathrm{Si}(\mathrm{Al}) \mathrm{CN}$ covalent ceramics. The microstructures of ceramics treated at different temperatures were observed. With Sarah, I used NMR to study the local chemical information of $\mathrm{Si}(\mathrm{Al}) \mathrm{CN}$ ceramics and their oxidation products. The ${ }^{27} \mathrm{Al}-\mathrm{MAS}$ NMR and ${ }^{28} \mathrm{Si}$-MAS NMR patterns of these materials were obtained. The results are great helpful to understand the structural change in the process of polymer to covalent ceramic transformation. The NMR results also give us the clues to better understand the extremely good oxidation resistance of these ceramics.
\end{abstract}

We start to prepare two journal papers one is Thermal Oxidation Behavior of Polymer-Derived Si(Al)CN Ceramics, which will be sent to Journal of the American Ceramic Society. The other is Structural Evolution of Polymer-Derived Si(Al)CN Ceramics, which will be presented to Chemistry of Materials. All of these results done at PNNL will be include in the two Journal papers. 


\title{
Detailed Hygroscopic Study of Sodium Nitrate Particles by SEM and FTIR
}

\author{
Zhiwei Yang \\ Department of Mechanical Engineering \\ University of Delaware \\ Newark, DE 19716, USA \\ Alexander Laskin \\ W.R. Wiley Environmental Molecular Sciences Laboratory, \\ Pacific Northwest National Laboratory, \\ PO Box 999, MSIN K8-88, Richland, WA 99352, USA \\ Hai Wang \\ Department of Aerospace and Mechanical Engineering, \\ University of Southern California, \\ Los Angeles, California 90089-1453
}

\begin{abstract}
The hygroscopic properties of $\mathrm{NaNO}_{3}$ and $\mathrm{NaNO}_{3} / \mathrm{NaCl}$ mixture particles were studied using Fourier Transform Infrared Spectroscopy and the morphology changes were imaged by Scanning Electron Microscopy. It was found that $\mathrm{NaNO}_{3}$ is very hygroscopic and will remain in aqueous form at relative humidity as low as less than $10 \%$. The implication to aged sea salt aerosol is that it will always has aqueous outer layer which has higher light scattering ability and will catalyze heterogeneous reaction at its gas-liquid surface.
\end{abstract}

\section{Introduction}

It is well-known that hygroscopic atmospheric aerosols not only can scatter sunlight directly back into space (direct effect), and they can also change could properties and affect the climate by acting as cloud condensation nuclei (indirect effect). In addition, they can participate or catalyze heterogeneous chemical reaction at the aqueous surface after absorbing water and the kinetic rate of reactions can be enhanced several magnitude $[1,2]$. For many hygroscopic aerosols, there exists a deliquescence phenomenon that the originally solid aerosols suddenly transit into liquid droplet as the relative humidity $(\mathrm{RH})$ increase up to a critical value. The droplets will grow gradually larger by further increasing RH. In the reverse direction, decreasing $\mathrm{RH}$, the droplets will remain in a supersaturated liquid form even $\mathrm{RH}$ is lower than the deliquescence point until at another critical RH the aerosols undergo efflorescence, not like bulk solution whose efflorescence happens at RH not far from that of deliquescence [3-5]. These hygroscopic hysteresis 
properties of aerosols have important effects on the air quality, visibility, radiative forcing, climate change and kinetic rate of related reactions.

Sodium nitrate, which was formed by reaction of sea salt with nitric acid, is a common component of aerosols in marine regions. It plays an important role in aged marine aerosols' properties such as light scattering. Yet only a few studies have been carried out to analyze the hygroscopic properties of sodium nitrate. Tang et al. [6-8] had used the single particle levitation or electrodynamic balance method to study $\mathrm{NaNO}_{3}$ and $\mathrm{NaCl} / \mathrm{NaNO}_{3}$ particles. Chung and Hsu [9] used a thermal conductivity detector to measure the vapor pressure, and they did not observe hysteresis effect on sodium nitrate. Gysel et al. used Tandem Mobility Analyzer to study sodium nitrate and also didn't catch the hysteresis effect.

Recently Hoffman et al. [10] used environmental scanning electron microscopy (ESEM) to monitor the morphological changes of sodium nitrate during hydration and dehydration. The conventional high vacuum SEM coupled energy dispersive analysis of $\mathrm{X}$-rays (SEM/EDX) was utilized to provide additional imaging and elemental information. And a long path Fourier Transform Infrared Spectroscopy (FTIR) was used to study the extent of water association of $\mathrm{H}_{2} \mathrm{O}$ and $\mathrm{NaNO}_{3}$ particles as a function of $\mathrm{RH}$ at $23^{\circ} \mathrm{C}$. The morphology of $\mathrm{NaCl} / \mathrm{NaNO}_{3}$ mixture particles, representative of aged sea salt particles in the atmosphere, was also studied with ESEM. And implication for aged sea salt aerosols was discussed. This work serves as a supplemental study which provides the detailed hygroscopic curves of $\mathrm{NaNO}_{3}$ and $\mathrm{NaCl} / \mathrm{NaNO}_{3}$ particles during hydration and dehydration process by FTIR, and images of the same cell studied by FTIR before and after hygroscopic studies.

\section{Experimental methods}

The schematic experimental setup of FTIR system is shown in Figure 1. The infrared absorption spectra were acquired by a Fourier Transform Infrared Microscope (Bruker, Model IFS 66/S) working in transmission mode and equipped with a MCT detector. The resolution is set at $4 \mathrm{~cm}^{-1}$ and the working range is $6000 \mathrm{~cm}^{-1}$ to $400 \mathrm{~cm}^{-1}$. The TEM grid was mounted into a sample stage (Linkam, Model THMS 600). The temperature in the sample chamber of the stage was maintained by a temperature controller (Linkam, Model TP 93). The relative humidity was provided by a portable dew point generator (LI-COR, Inc., Model LI-610) and monitored after the sample stage by a calibrator (Vaisala, Model HMP234). The pure nitrogen gas was used as a carrier for water vapor. In the normal operation, Valve 1 was closed and Valve 2 was open. The temperature of the sample stage was fixed at $23{ }^{\circ} \mathrm{C}$. The desired relative humidity was obtained by set the temperature of dew point generator to a corresponding value calculated according to Buck's formula [11]. By switching off the vapor flow (Valve 2) from the generator and switching on valve 1, close to zero relative humidity can be established in the sample stage.

An FEI XL30 digital field emission gun SEM was utilized in this study. $\mathrm{NaNO}_{3}$ particles used in this study was prepared by atomizing a solution of either $\mathrm{NaNO}_{3}$ or an $\mathrm{NaNO}_{3} / \mathrm{NaCl}$ mixture of designed ratio onto a 400 mesh carbon type-B TEM grid (Ted Pella, Inc.). For SEM imaging, $20 \mathrm{keV}$ accelerating voltage and $10 \mathrm{~mm}$ working distance 
were adopted. The image was analyzed by Genesis ${ }^{\circledR}$ software (EDAX Inc.) and the size of particles was obtained.

\section{Results and Discussion}

$\mathrm{NaNO}_{3}$ particles on one cell of the TEM grid was imaged by SEM before and after hydration process in FTIR study, as seen in Figure 2a, 2b. By selecting a $600 \mu \mathrm{m}$ secondary aperture in FIIR microscope, we can obtain the IR spectrum of particles on the same cell imaged by SEM. Water vapor was provided by passing pure nitrogen gas through the dew point generator. After hydration process, some of the smaller particles merged together or with nearby larger particles and formed larger particles, as seen Figure 2c, 2d. The mean diameters of $\mathrm{NaNO}_{3}$ particle before and after hydration process are $1.1 \mu \mathrm{m}$ and $1.4 \mu \mathrm{m}$ with standard deviation 0.7 and 1.3 , respectively. The advantage of this work over previous studies is the potential ability to examine particle size influence on IR spectrum.

The $\mathrm{NaNO}_{3}$ particles remain unchanged until the relative humidity reach $60 \%$, as shown in Figure 3. The absorption spectrum had been smoothed to reduce the noise of water vapor absorption. Above $60 \% \mathrm{RH}, \mathrm{NaNO}_{3}$ particles begin to absorb water and the $\mathrm{OH}$ absorption peak of liquid water starts to appear. At $71 \% \mathrm{RH}$, water absorption of $\mathrm{NaNO}_{3}$ particles rise drastically. At $73 \% \mathrm{RH}, \mathrm{NaNO}_{3}$ particles reach deliquescence and become aqueous droplets completely. Further increasing RH, the droplets grow slower, as seen in the slow-down increase of $\mathrm{OH}$ absorption peak at $75 \% \mathrm{RH}$.

In this experiment, we examined the hygroscopic properties of $\mathrm{NaNO}_{3}$ particles in the hydration process that relative humidity increases gradually from $0 \%$, and the dehydration process that relative humidity decreases from a $\mathrm{RH}$ higher than the deliquescence point. The $\mathrm{OH}$ absorption peak area was plotted against relative humidity, and shifted linearly with respect to the results obtain in Tang and Fung's work by electrodynamic balance method. It can be seen that the results agree well with each other, except that in our case we didn't observe efflorescence until relative humidity close to 1

$\%$. Considering the facts that their particle size is 14 to $16 \mu \mathrm{m}$ in diameter while the mean diameter in our study is $1.4 \mu \mathrm{m}$, the difference in dehydration process suggests the size of particles play an important effect on efflorescence. After the relative humidity reaching 1 $\%$, we did the hydration and dehydration process again, as shown by the solid symbols. And these processes follow that of the efflorescence curve which means there is still water associated with the particles. By comparing to electrodynamic balance method, there is about 1 molecules of water per three molecules of solutes.

As mention above, sodium nitrate is formed in marine aerosols by the reaction of sea salt and nitric acid. The result that sodium nitrate particles do not effloresce means that sodium nitrate containing sea salt aerosols will always remain aqueous form and the light scattering ability will be significantly increased compare to crystal sea salt particles. If the reaction of sea salt with nitric acid does not complete, it will result in aerosols with a mixture of $\mathrm{NaNO}_{3} / \mathrm{NaCl}$. As shown in the SEM picture in previous work [], the $\mathrm{NaCl}$ will be located in the center of the particle and $\mathrm{NaNO}_{3}$ in the outer layer. To consider the detailed hygroscopic properties of sea salt aerosol resulted from incomplete reaction, we carried out the studies of particles composed of the mixture of $\mathrm{NaNO}_{3} / \mathrm{NaCl}$ at molar ratios of $3: 1,1: 1$, and $1: 3$. 
The particles of 3:1 ratio of $\mathrm{NaNO}_{3} / \mathrm{NaCl}$ exhibited similar hydration behavior from the initial crystal form, as seen in Figure 5. In the dehydration process, the particles effloresced at $31 \% \mathrm{RH}$. We reduced the relative humidity to $25 \%$ and then increased it again. The results showed that the water absorption peak area did not follow the first hydration curve or dehydration curve, and the particles began to absorb water vapor below $40 \% \mathrm{RH}$. It means that the outer layer $\mathrm{NaNO}_{3}$ of particles did not effloresce.

When the mixture ratios of $\mathrm{NaNO}_{3} / \mathrm{NaCl}$ changes from 3:1 to 1:1, the particle underwent effloresce at $38 \% \mathrm{RH}$, when is higher than the previous mixture. And the second time hydration curve was lower than the previous one, which is reasonable by considering the different hygroscopic properties of $\mathrm{NaNO}_{3}$ and $\mathrm{NaCl}$. There are more features in the efflorescence process that it takes two steps to efflorescence. The first step is that $\mathrm{NaCl}$ crystallize out in the supersaturated solution. The second step may be $\mathrm{NaCl}$ inducing $\mathrm{NaNO}_{3}$ to effloresce partly in the outer aqueous layers. But $\mathrm{NaNO}_{3}$ never completely effloresce, as seen in Figure 6.

Further reducing $\mathrm{NaNO}_{3}$ component in the mixture, we see that the second hydration curve of is just slightly higher than the first hydration curve. However, even the relative humidity was reduced as low as the outer layer $\mathrm{NaNO}_{3}$ was still remain uneffloresced.

\section{Conclusion}

$\mathrm{NaNO}_{3}$ has important effects on the hygroscopic properties of aged sea salt aerosol in that it will remain in aqueous state in the atmosphere which as a typical relative humidity from $20 \%$ to $90 \%$. And hence it has higher lighter scattering capability than the crystal aerosol. It will also facilitate the heterogeneous reaction at the gas-liquid interface which is significantly higher than the gas-solid interface.

\section{Acknowledgements}

This work was support by the Interfacial and Condensed Phase Chemical Physics Summer Research Institute at Pacific Northwest National Laboratory.

1. A. Laskin, D.J.G., W. Wang, S. W. Hunt, J. P. Cowin, S. D. Colson, B. J. Finlayson-Pitts, Reactions at interfaces as a source of sulfate formation in seasalt particles. Science, 2003. 301: p. 340-344.

2. M. Ammann, M.K., D. T. Jost, L. Tobler, E. Rössler, D. Piguet, H. W. Gäggeler, U. Baltensperger, Heterogeneous production of nitrous acid on soot in polluted air masses. Science, 1998. 395: p. 157-160.

3. C. Orr Jr., F.K.H., and W. J. Corbett, Aerosol size and relative humidity. Journal of Colloid Science, 1958. 13: p. 472-482.

4. Tang, I.N., Deliquescence properties and particle size change of hygroscopic aerosols. Generation of Aerosols, edited by K. Willeke, Butterworth-Heinemann, Newton, MA, 1980: p. chap. 7. 
5. M. J. Rood, M.A.S., T. V. Larson, and D. S. Covert, Ubiquitous nature of ambient metastable aerosols. Nature, 1989. 337: p. 537-539.

6. Munkelwitz, I.N.T.a.H.R., Water activities, densities, and refractive indices of aqueous sulfates and sodium nitrate droplets of atmospheric importance. Journal of Geophysical Research, 1994. 99: p. 18801-18808.

7. Tang, I.N., Thermodynamic and optical properties of mixed-salt aerosols atmoshperic importance. Journal of Geophysical Research, 1997. 102: p. 18831893.

8. I. N. Tang, a.K.H.F., Hydration and Raman scattering studies of levitated microparticles: $\mathrm{Ba}(\mathrm{NO} 3) 2$, $\mathrm{Sr}(\mathrm{NO} 3) 2$, and $\mathrm{Ca}(\mathrm{NO} 3) 2$. Journal of Chemical Physics, 1997. 106(5): p. 1653-1660.

9. $\quad$ C.-T. Lee, W.-C.H., The measurement of liquid water mass associated with collected hygroscopic particles. Journal of Aerosol Science, 2000. 31(2): p. 189197.

10. R. C. Hoffman, A.L., and B. J. Finlayson-Pitts, Sodium nitrate partices: physical and chemical properties during hydration and dehydration, and implications for aged sea salt aerosols. Journal Aerosol Science, 2004. 35: p. 869-887.

11. Buck, A.L., New equations for computing vapor pressure and enhancement factor. J. Appl. Meteor., 1981. 20: p. 1527-1532. 


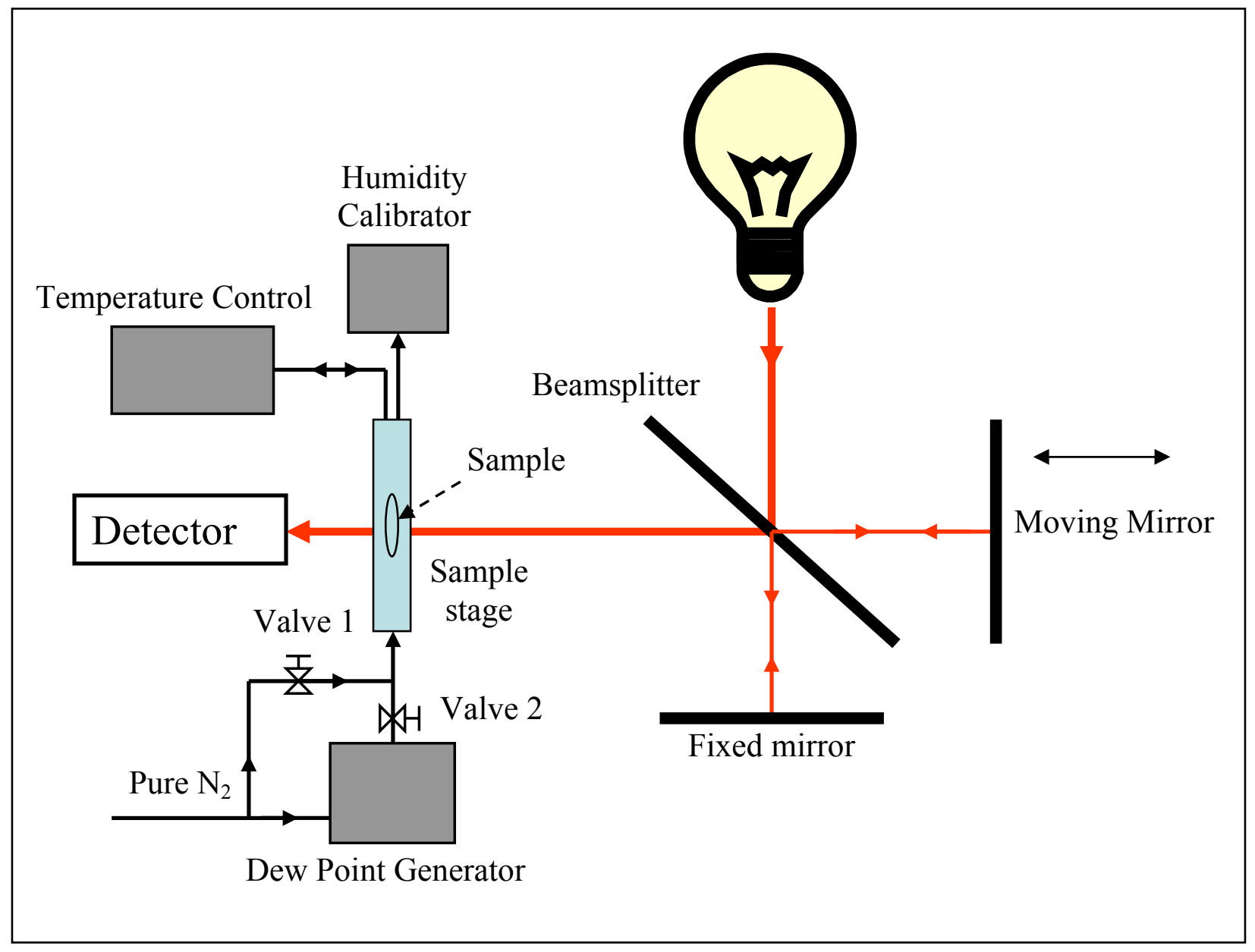

Figure 1 
(a)

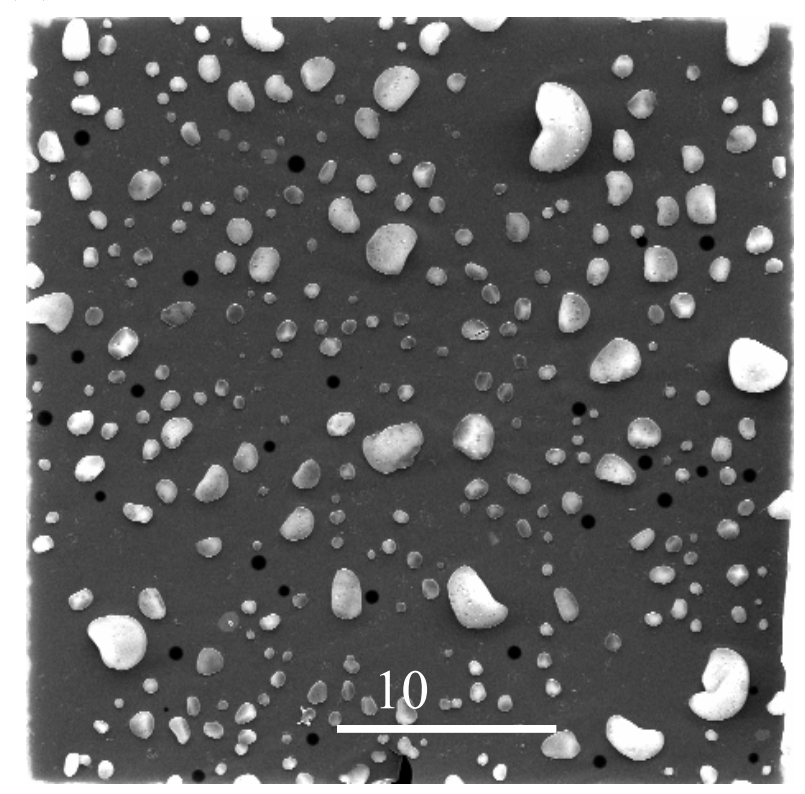

(c)

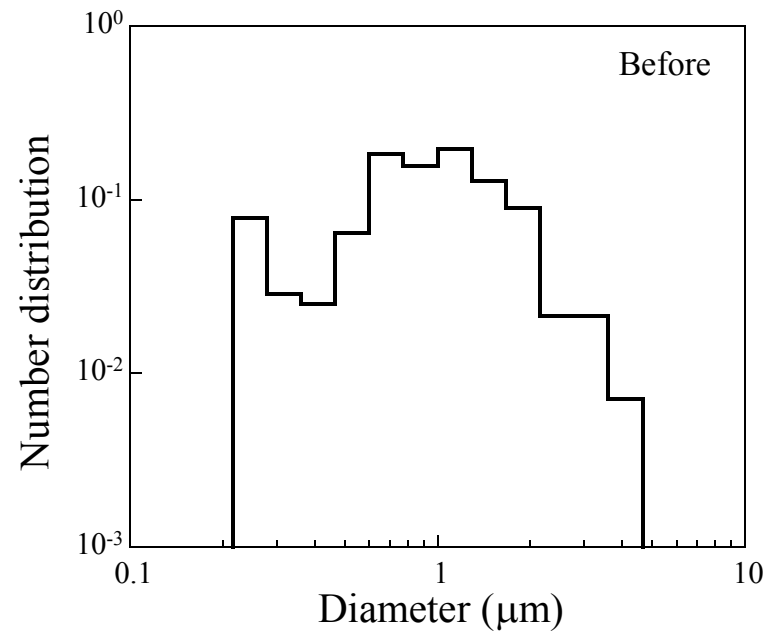

(b)

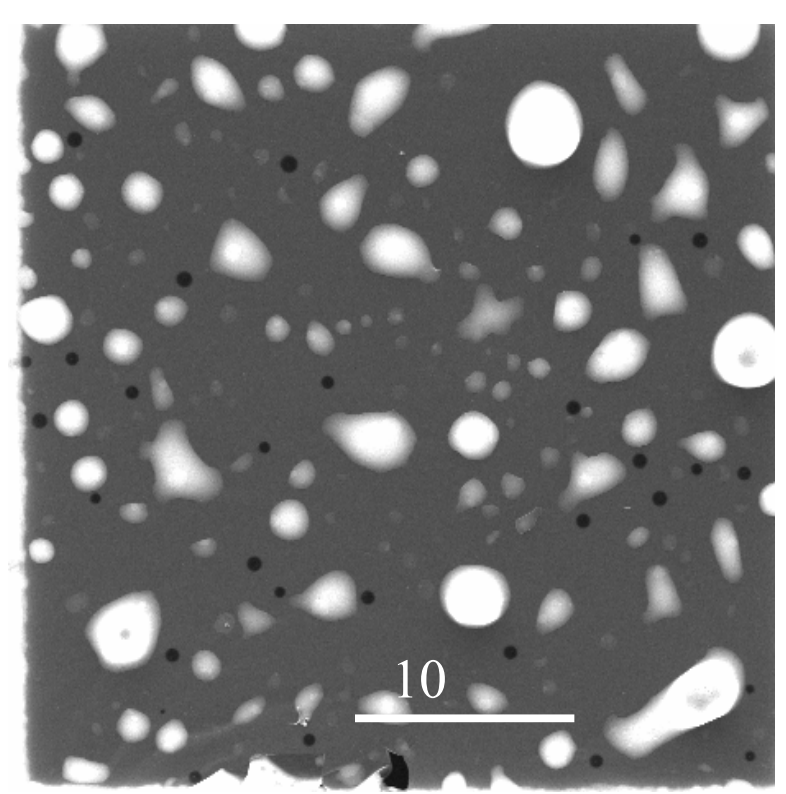

(d)

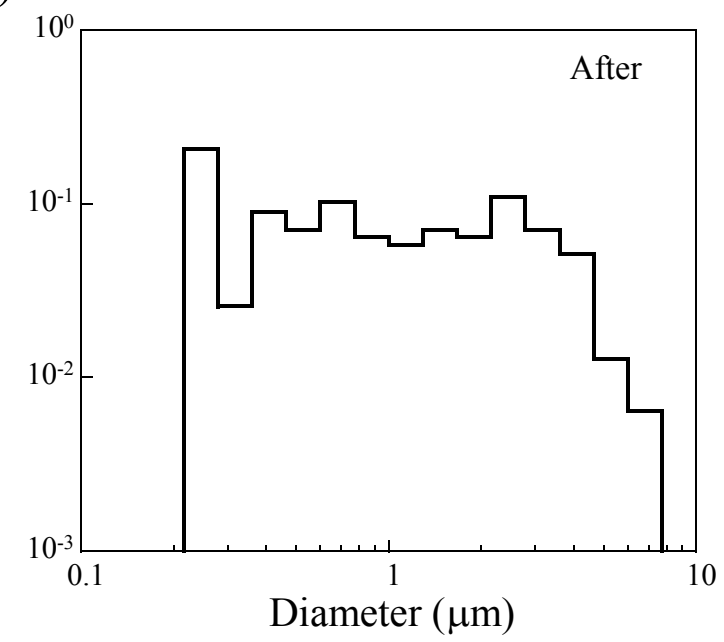

Figure 2 


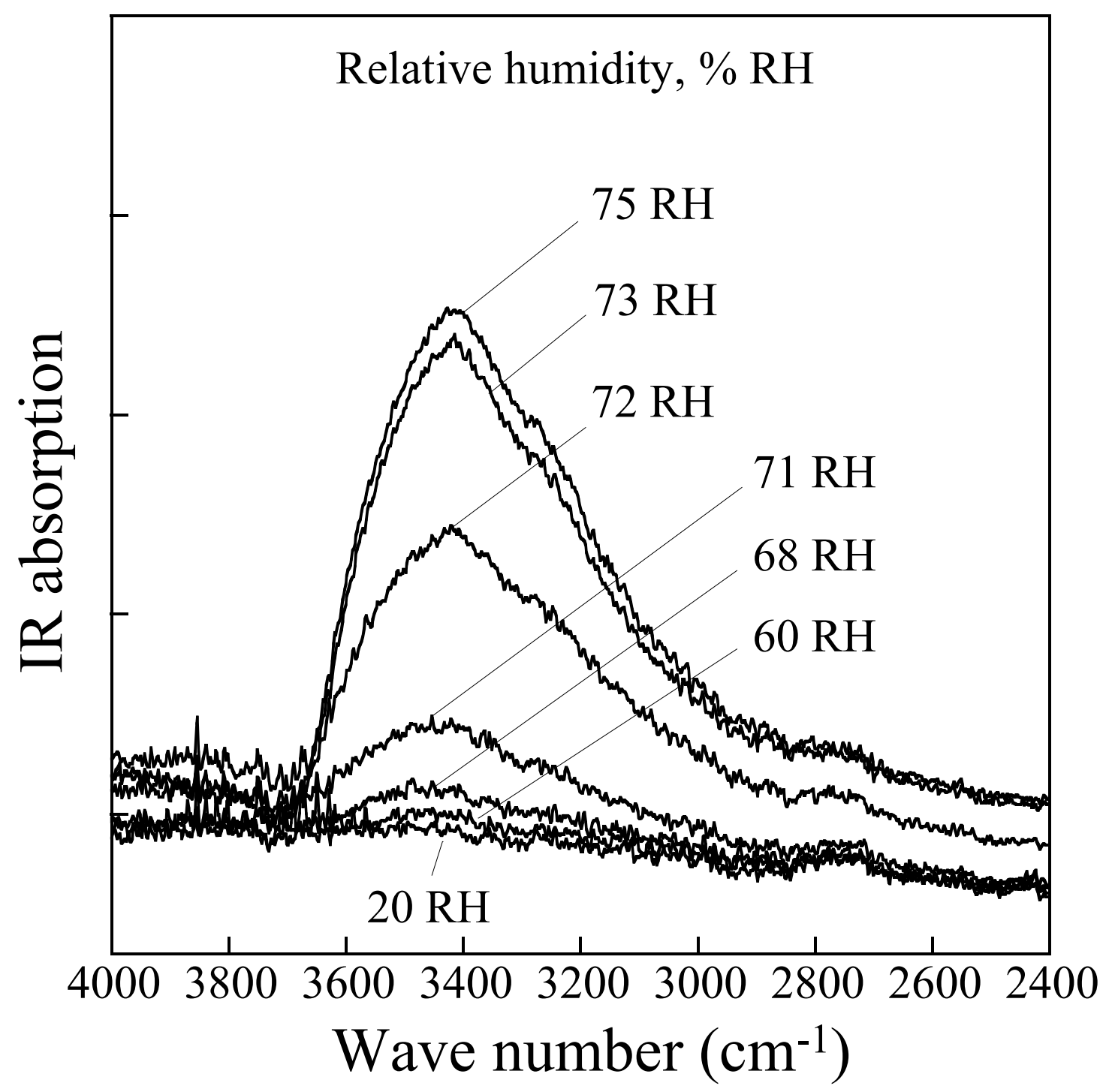

Figure 3 


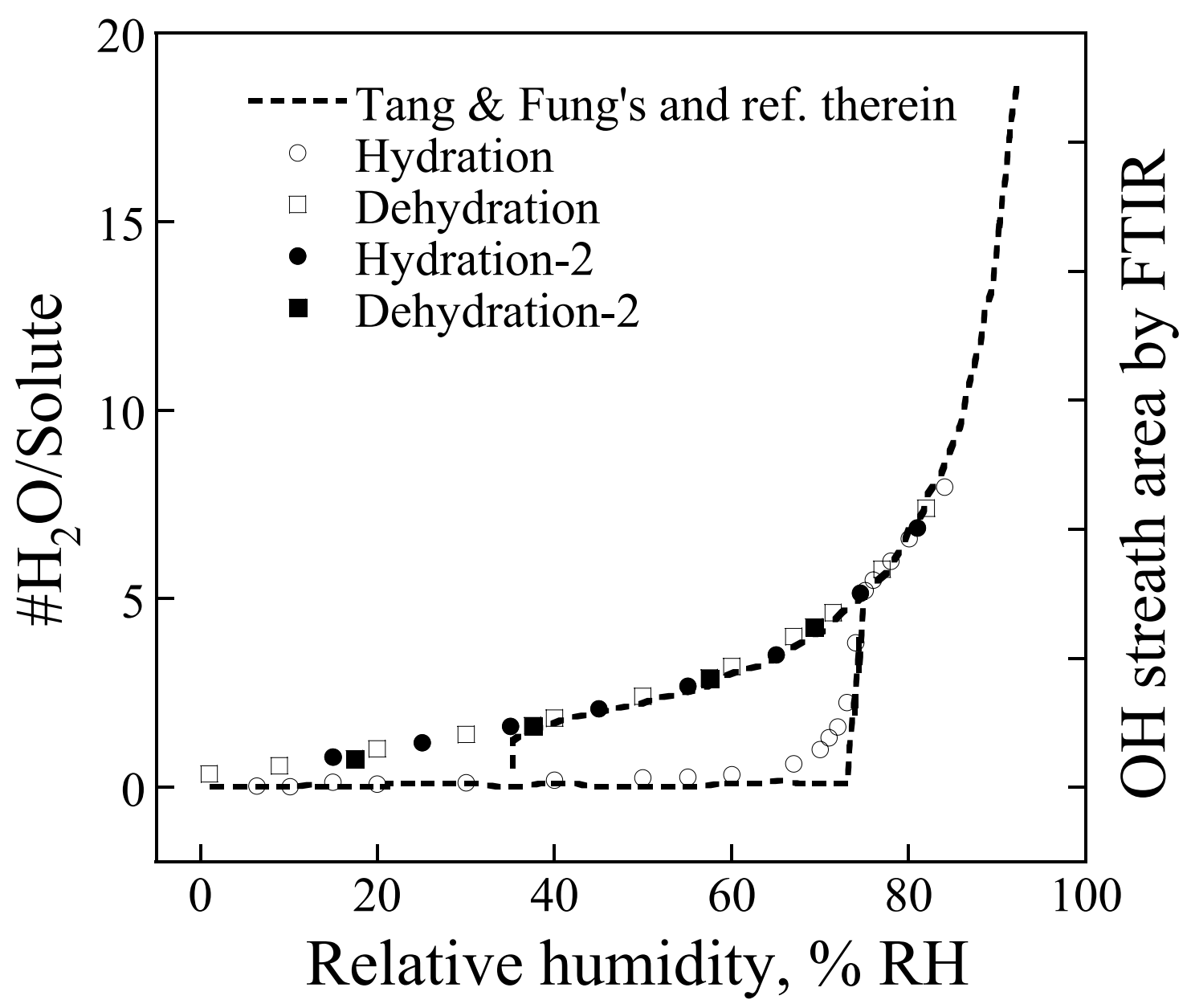

Figure 4 


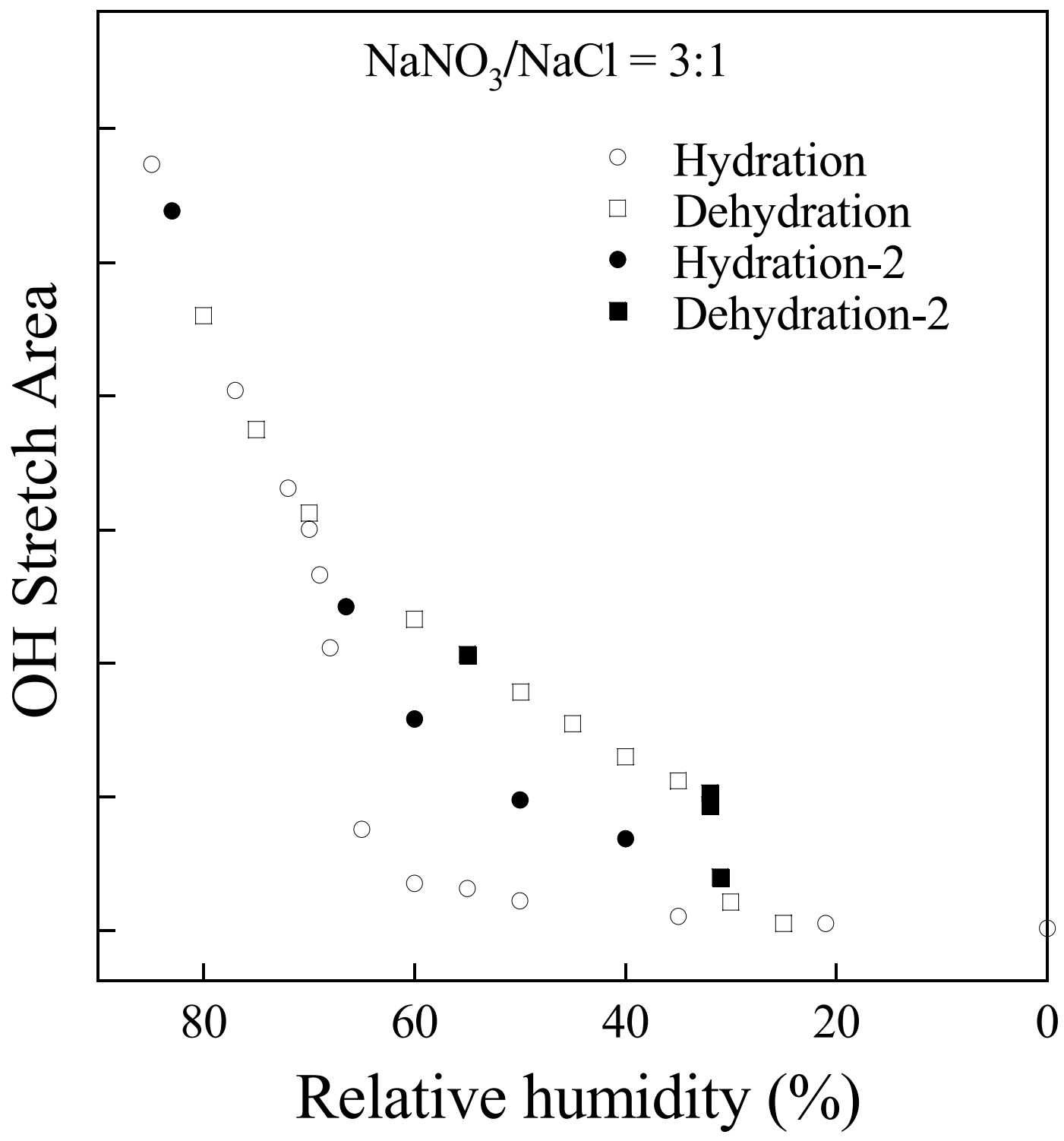


Figure 5

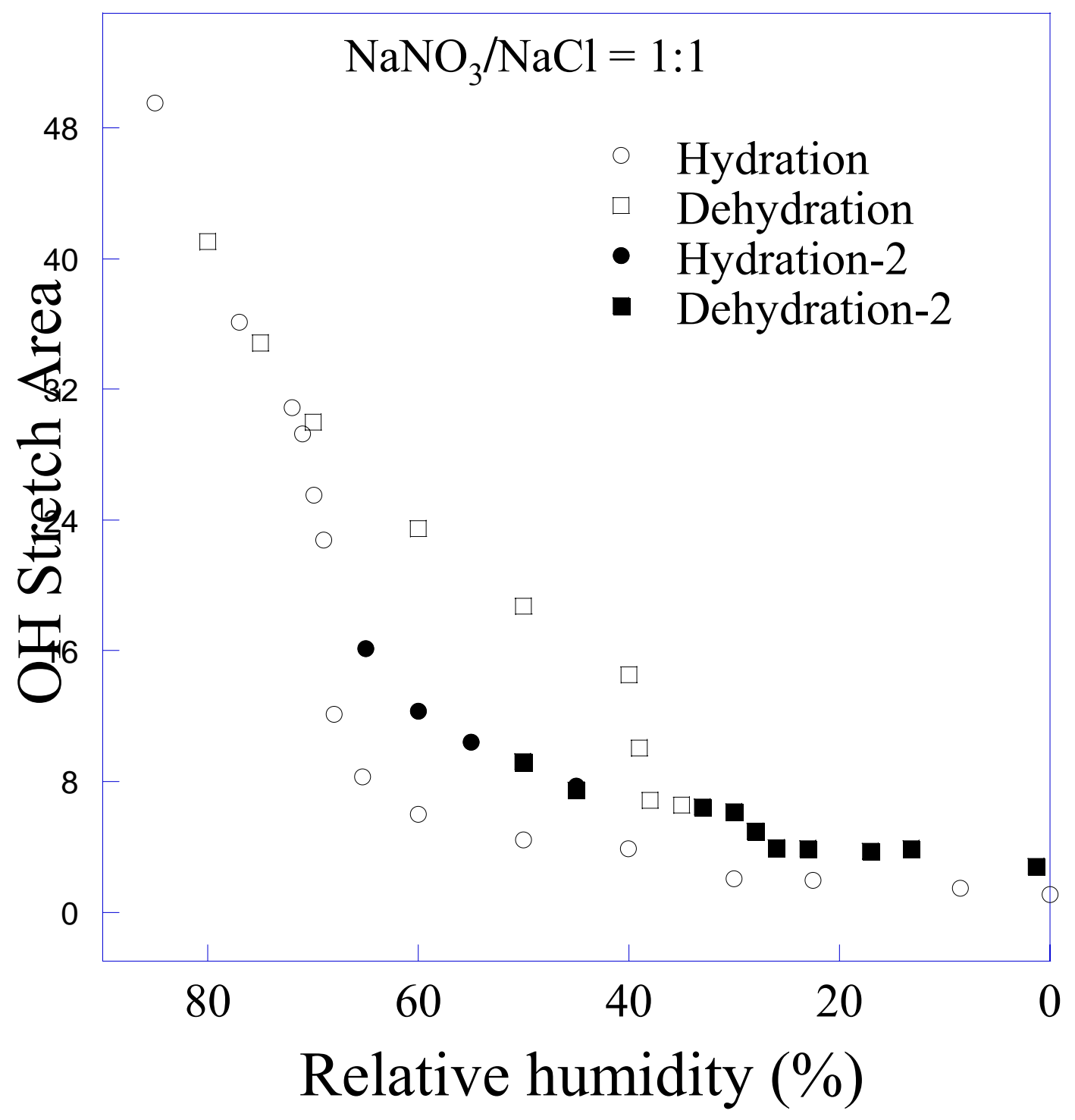




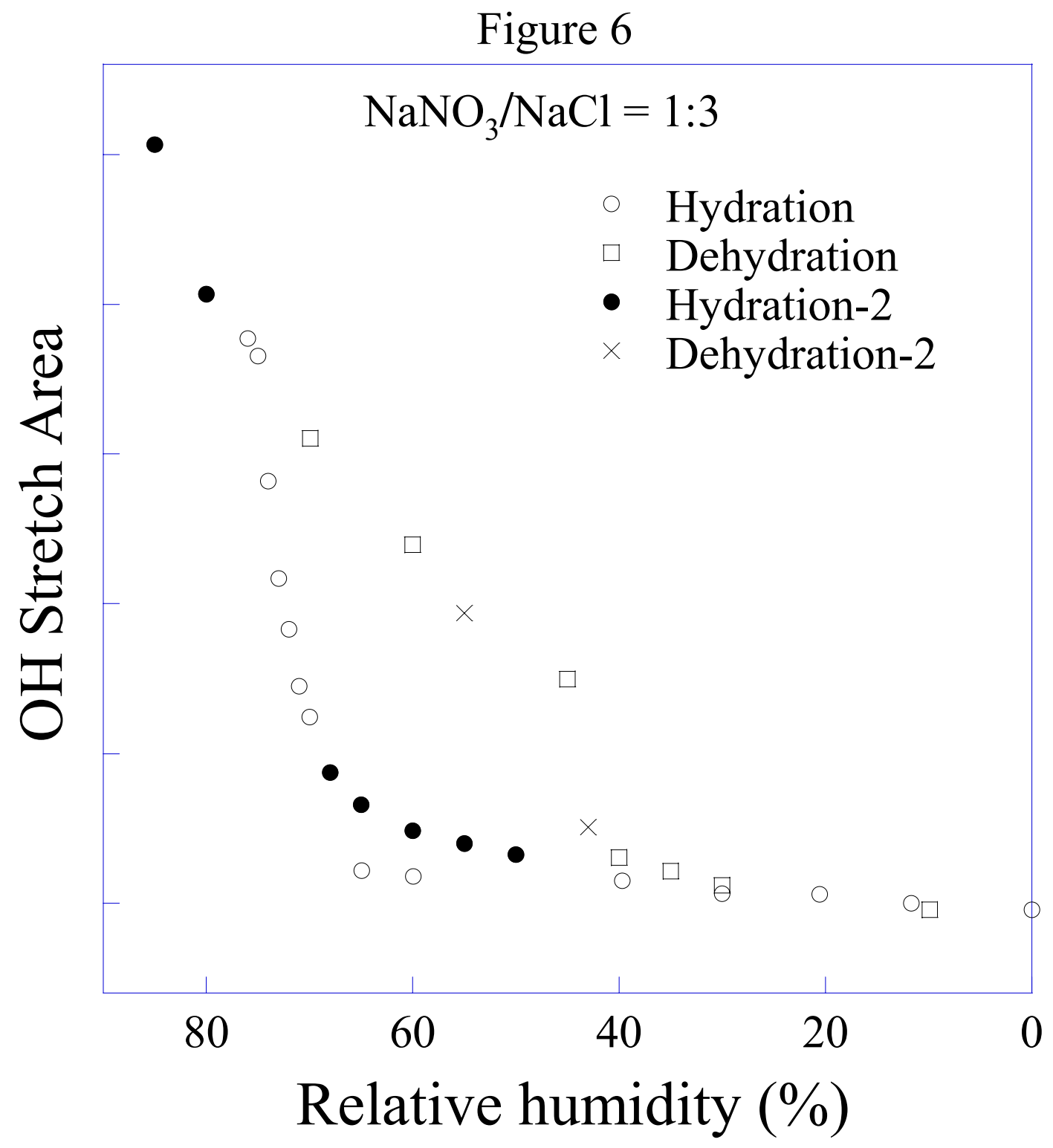

Figure 7 


\title{
Chemical Characterization of Phosphorous Containing Soot Particles
}

\author{
Z. Yang ${ }^{3}$, A. Laskin ${ }^{1}$, B.G. Bunting ${ }^{2}$, J. P. Cowin ${ }^{1}$,

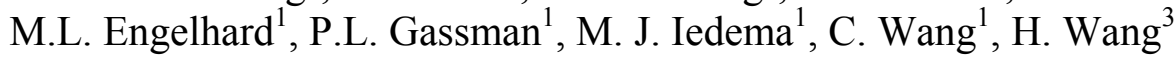 \\ ${ }^{1}$ William R. Wiley Environmental Molecular Sciences Laboratory, \\ Pacific Northwest National Laboratory, Richland, WA 99352 \\ ${ }^{2}$ National Transportation Research Center, \\ Oak Ridge National Laboratory, Knoxville, TN 37932 \\ ${ }^{3}$ Department of Mechanical Engineering, \\ University of Delaware, Newark, DE 19716
}

(Result presented at the 2004 DEER conference)

Zinc dialkyldithiophosphate (ZDDP) was used for over 50 years in lubricating oil as antioxidant and antiwear additive. Engine oil can enter combustion chamber through many routes: oil film formed on the cylinder liners' surface, pushing up effect by movement of pistons and rings, by blow-by gas, or from the valve guides and other components. And phosphorus had long been found to decrease the activity of automotive catalysts, affect the performance of oxygen sensor, and result in a degradation of emission control.

This work is to examine the chemical properties of phosphorous containing soot which may link engine characteristics, additive chemistry, and catalyst poisoning as well as environmental effects.

We have collected soot samples in the exhaust of the Engine Test Stand in Oak Ridge National Laboratory at three different working conditions: varying consumption rate of lube oil doped with ZDDP, varying load with ZDDP doping, varying load without ZDDP doping. Here as a preliminary study we focus on the study of soot in the series of varying load with ZDDP doping. The sampled was collected with Carbon Type-B TEM grid (Ted Pella, Inc.) by a Time-Resolved Aerosol Collector which was developed at W.R. Wiley Environmental Molecular Sciences Laboratory of Pacific Northwest National Laboratory.

Scanning Electron Microscopy (SEM), Transmission Electron Microscopy (TEM), Computer-Controlled SEM with Energy Dispersed detection of X-rays (CCSEM/EDX), X-ray Photoelectron Spectroscopy (XPS), and Fourier Transform Infrared Spectroscopy were used characterize the phosphorous containing soot. The advantage of the sampling technique with TEM grid was seen in that it fitted all the above experimental techniques, and more will be discussed later.

From the SEM images of soot samples of $2 \%, 29 \%, 57 \%$ and $93 \%$ loads, it shows marked difference in the particle morphology. At 2\% load, there may be unburned oil collected on the substrate. With increasing load, the morphology of soot particles changes from dispersive distribution to more aggregate distribution. TEM images provides a more 
detail information about particles. It shows that phosphorous containing soot particles changes from amorphous form into crystallized form, as seen in the images of particles of $2 \%$ and $93 \%$ loads.

With CCSEM/EDX, particle morphology changes can be quantified in aspect ratio and size distribution, and particle composition can be examined. From the CCSEM/EDX results, it can be seen that particle distribution is wider in higher load case. In the ternary plots, the most evident change of particle composition is that the sulfur content reduced drastically from $2 \%$ to $93 \%$ loads.

XPS data yields the chemical bond energy information of elements. From XPS results, it can be seen that phosphorous and zinc's chemical state changed from $2 \%$ to 93\% loads. Further experimental techniques such as Mass Spectroscopy are needed to identify the compounds that phosphorous and zinc formed in soot particles at different loads.

We used FTIR to study the hygroscopic properties of phosphorous containing soot particles. The sample grid was imaged before and after FTIR study by SEM. By carefully selecting the secondary aperture on the FTIR microscopy, we were able to study the same cell on the TEM grid that had been imaged by SEM. The sample stage was kept at $23^{\circ} \mathrm{C}$ by a temperature controller. A dew point generator was used to supply water vapor of given relative humidity. FTIR studied showed that for soot at different loads, there are marked differences in the water absorption spectrum when changing relative humidity and characteristic spectrum at zero relative humidity. However, the fresh phosphorous containing soot is generally hydrophobic.

The aging of phosphorous containing soot needs to be studied to understand their overall environmental impact, since fresh soot released into atmosphere will be oxidized gradually during their lifetime in the air. 


\title{
Understanding Chemical Processes of Bioactive Glasses
}

\author{
Todd Zeitler and Alastair N. Cormack \\ Ceramic Science Department \\ Alfred University School of Engineering \\ Alfred, NY \\ And \\ L. Rene Corrales \\ Pacific Northwest National Laboratory \\ Richland, WA
}

\section{Introduction}

The objective of my work this summer at PNNL was to augment my atomic-scale classical molecular dynamics (MD) study of the reaction of water with the surface of a bioactive glass with DFT quantum calculations, making use of the expertise, software (e.g., NWCHEM), and computing power (e.g., MPP2) available at PNNL. In particular, I planned to study the energetics of an exchange of a proton $\left(\mathrm{H}^{+}\right)$for $\mathrm{Na}^{+}$(which is native to the glass), creating an $\mathrm{OH}$ group on the glass surface; this is the initial reaction when this type of glass comes in contact with water or body fluid. An ensuing reaction, whereby an Si-O-Si or Si-O-P bridge is broken by the introduction of water, causes dissolution of the glass structure, and can also be studied using this technique. These interactions with water are described more accurately using the quantum mechanical calculations of DFT. Using NWCHEM will result in more precise energy calculations and structures.

Because it takes into account all the electrons for each atom in the simulation, and to avoid huge computational costs, a DFT calculation requires significantly fewer atoms than an MD calculation. To generate a suitable structure for a DFT calculation, a smaller MD simulation is run. The original MD simulation consisted of 2835 atoms while the one for the DFT calculation has 94 atoms. This study focused on Bioglass ${ }^{\circledR}$ (45S5 glass) of approximate composition $46.1 \mathrm{SiO}_{2} \circ 2.6 \mathrm{P}_{2} \mathrm{O}_{5} \circ 24.4 \mathrm{Na}_{2} \mathrm{O} \circ 26.9 \mathrm{CaO}$ (actual compositions given in Table I). Simulation box sizes were calculated to give a density of $2.66 \mathrm{~g} / \mathrm{cm}^{3}$. This glass is extremely bioactive - it reacts very quickly with the body to generate a hydroxylapatite layer that aides in quick bone growth. Two other compositions have been studied already with $\sim 3000$-atom simulations. 


\section{Procedure}

Initially, two approaches were taken to get a suitable structure for the DFT calculation: 1) a random configuration of 94 atoms and 2) extraction of a piece of the surface previously studied by MD (2835 atoms) that is 'frozen' through the melt and quench process.

In the second approach, a phosphorus atom with an NBO that had been hydrated in a previous 2835 -atom simulation was selected, as well as all of the atoms within a $3 \AA$ radius. It was placed as the center of the (bulk) simulation box, and the remaining atoms were randomly put around the 'cluster'. The cluster was immobilized during the melt and quench portions of the creation process, and later allowed to relax. When the bulk was 'cut' to make a surface, the cut was made so that the phosphorus (and its bonded oxygen) was in a similar orientation as the final configuration studied in the MD study.

The 2835-atom simulation has been thoroughly studied with respect to the surface reaction with water. The 8505 -atom simulation was later added as a check for the effect of simulation size on network fragment sizes (discussed later) and was conducted identically to the 2835-atom simulation. The 376-atom simulation was added as an intermediate simulation between the 2835- and 94-atom simulations and was conducted identically to the 94-atom simulation.

Table I. Composition of $8505,2835,376$, and 94 -atom MD simulations (in mol\% and number of atoms).

\begin{tabular}{|c|c|c|c|c|c|}
\hline (mol\%) & $\mathrm{SiO}_{\mathbf{2}}$ & $\mathbf{P}_{\mathbf{2}} \mathbf{O}_{\mathbf{5}}$ & $\mathbf{N a}_{\mathbf{2}} \mathbf{O}$ & $\mathbf{C a O}$ & box size $(\mathbf{\AA})$ \\
\hline $\begin{array}{c}\text { 8505-atom } \\
\text { 2835-atom }\end{array}$ & 46.10 & 2.60 & 24.40 & 26.90 & 48.68 \\
\hline 376-atom & 45.45 & 3.60 & 24.40 & 26.90 & 33.75 \\
\hline 94-atom & 45.45 & 3.03 & 24.24 & 27.27 & 17.13 \\
\hline
\end{tabular}

\begin{tabular}{|c|c|c|c|c|c|}
\hline (\# atoms) & $\mathbf{O}$ & $\mathbf{S i}$ & $\mathbf{P}$ & $\mathbf{N a}$ & $\mathbf{C a}$ \\
\hline 8505-atom & 4695 & 1383 & 156 & 1464 & 807 \\
\hline 2835-atom & 1565 & 461 & 52 & 488 & 269 \\
\hline 376-atom & 208 & 60 & 8 & 64 & 36 \\
\hline 94-atom & 52 & 15 & 2 & 16 & 9 \\
\hline
\end{tabular}

From comparing the resultant bulk TDF to the 2835 -atom simulation, this approach looked promising; however, it was later noticed that on surface creation, the 94- 
atom simulation became very 'hot' (in excess of $2000 \mathrm{~K}$ ). In an attempt to stabilise the system, the NPT ensemble was used; however, this resulted in the severe and continued shrinking of the simulation box. Many new simulation routes (i.e., combinations of NVE, NVT, NPT at different temperatures) were tested for their ability to produce stable bulk and surface structures. For simplicity's sake, this cluster approach was abandoned for the completely random configuration approach.

The simulation sequence that finally produced a reasonable structure is given in Table II and is compared to that of the 2835-atom MD simulation. The change in procedure that seemed to make the most difference was reducing the number of timesteps between updates to the Verlet neighbour list from 5 to 1 . All simulations were done with identical Buckingham potential parameters and using DL_POLY software.

Table IIa. Simulation sequence for 2835 -atom surface creation.

\begin{tabular}{|c|c|c|c|}
\hline \multicolumn{4}{|c|}{ 2835-atom and 8505-atom MD } \\
\hline ensemble & temp. (K) & teps (equilibrated) & other \\
\hline NVT & 6000 & 20000 & \\
\hline NVT & $6000-300$ & 285000 & linear quench \\
\hline NVT & 300 & $25000(5000)$ & TDF; BAD \\
\hline \multicolumn{4}{|c|}{ surface creation; addition of $20 \AA$ of vacuum } \\
\hline \multicolumn{4}{|l|}{$25 \%$ immobilised } \\
\hline NPT & 1500 & 80000 & anneal \\
\hline NPT & 300 & 80000 & \\
\hline \multicolumn{4}{|l|}{$50 \%$ immobilised } \\
\hline NPT & 300 & 80000 & ZDP \\
\hline
\end{tabular}

Table IIb. Simulation sequence for 94-atom surface creation.

\begin{tabular}{|c|c|c|c|}
\hline \multicolumn{4}{|c|}{ 94-atom and 376-atom MD } \\
\hline ensemble & temp. (K) & \#tsteps (equilibrated) & other \\
\hline NVT & 6000 & 20000 & \\
\hline NVT & $6000-300$ & 285000 & linear quench \\
\hline NVT & 300 & $25000(5000)$ & \\
\hline NVE & 300 & $100000(10000)$ & \\
\hline NPT & 300 & $500000(250000)$ & TDF; BAD \\
\hline NVT & $300-0$ & $15000(15000)$ & linear quench \\
\hline NPT & 'zero' & 50000 & \\
\hline $25 \%$ immobilised & & & \\
\hline NVE & & & \\
\hline NPT & 'zero' & 80000 & \\
\hline
\end{tabular}




\begin{tabular}{|l|c|c|l|}
\hline NPT & 300 & 80000 & \\
\hline NVT & 500 & 500000 & anneal \\
\hline NPT & 300 & 80000 & ZDP \\
\hline
\end{tabular}

\section{Results and Discussion}

Snapshots of the final bulk and surface structures are shown in Figures 1-6. Selected TDF (total distribution function), BAD (bond angle distribution) and ZDP (Zdensity profile) were calculated (Figures 7-12). TDF and BAD were calculated for the bulk structures and ZDP for the final surface structures; all were calculated over the final 20000 timesteps (400 configurations, 50 timesteps apart) of the simulations indicated in Table II. 


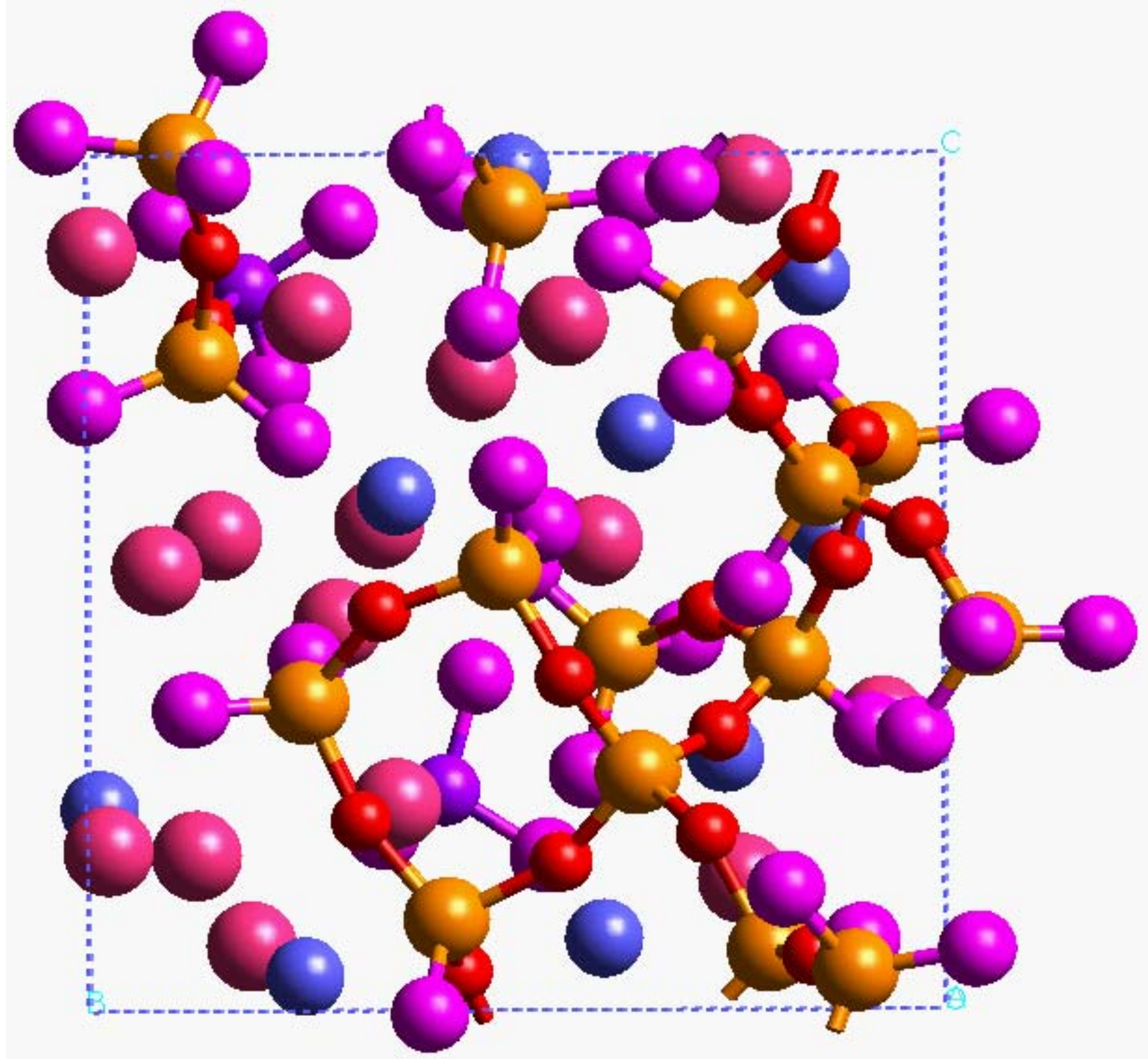

Figure 1. Bulk structure of 45S5 (94-atoms) glass (yellow=4-coordinated $\mathrm{Si}$; green=3coordinated $\mathrm{Si}$; red=BO; magenta $=\mathrm{NBO}$; turquoise $=$ free $\mathrm{O}$; purple $=\mathrm{P}$; blue $=\mathrm{Ca}$ ). 


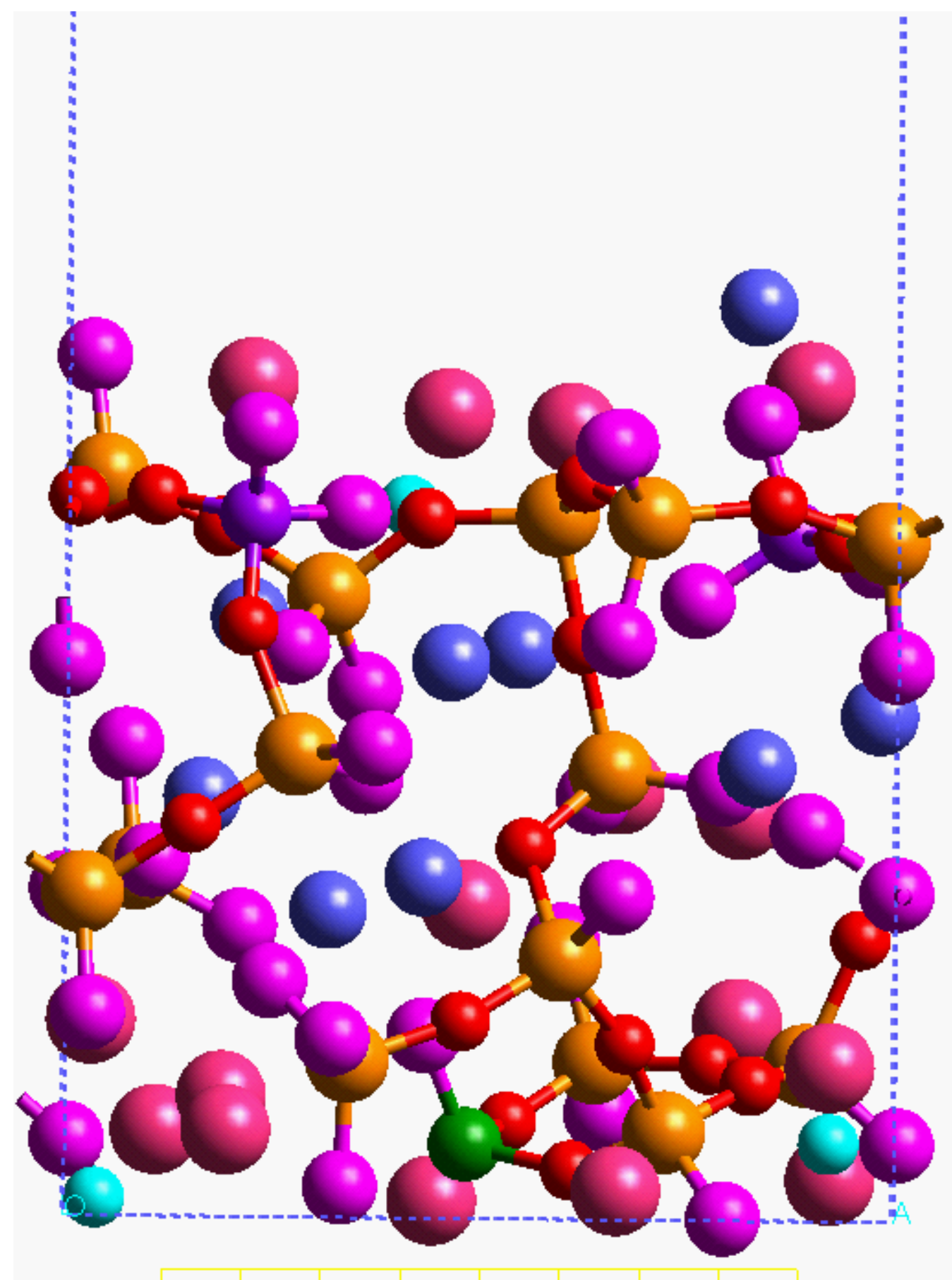

Figure 2. Surface structure of 45S5 (94-atoms) glass (yellow=4-coordinated $\mathrm{Si}$; green $=3$ coordinated $\mathrm{Si}$; red $=\mathrm{BO}$; magenta $=\mathrm{NBO}$; turquoise $=$ free $\mathrm{O}$; purple $=\mathrm{P}$; blue $=\mathrm{Ca}$ ). 


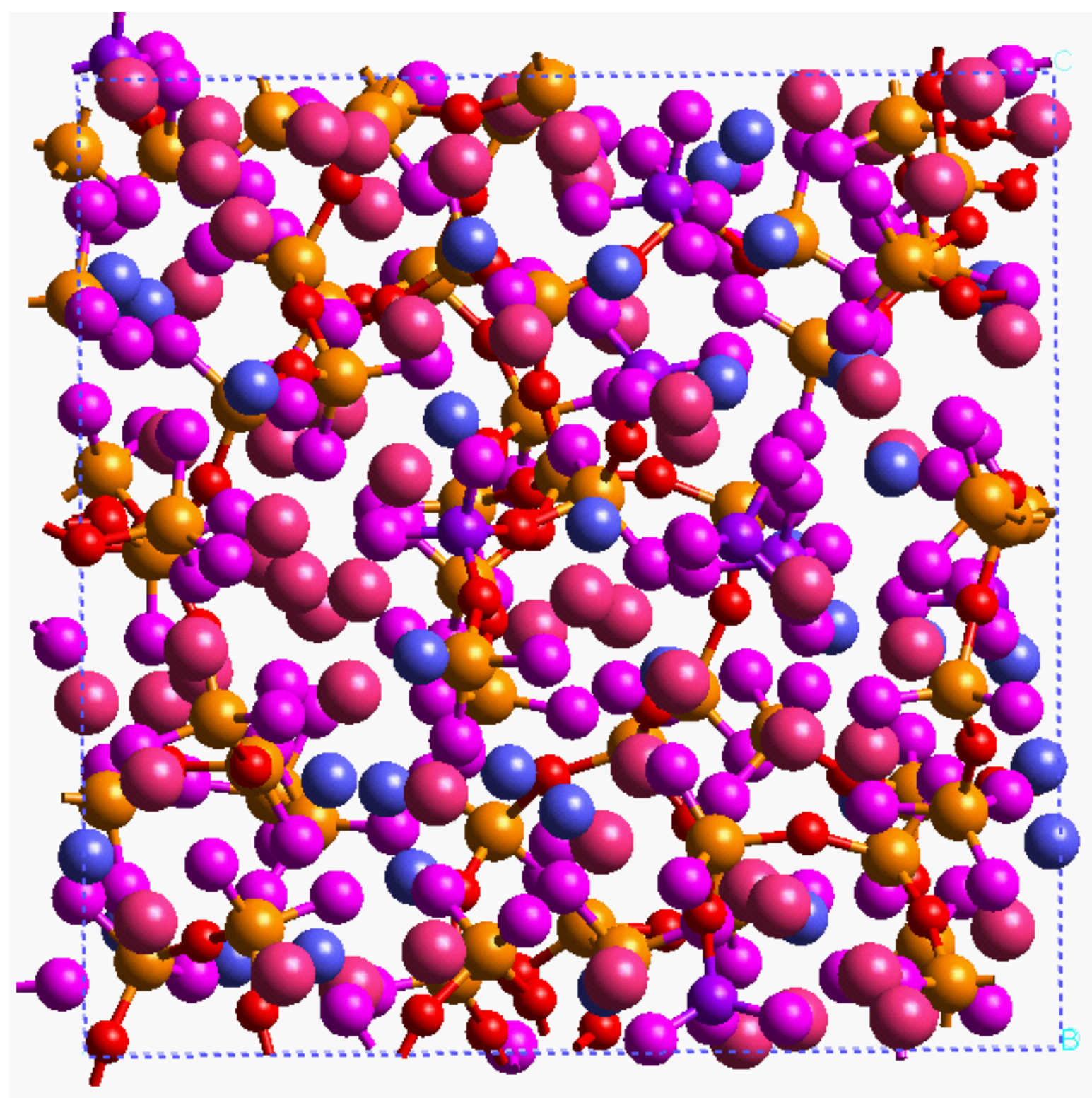

Figure 3. Bulk structure of 45S5 (376-atoms) glass (yellow=4-coordinated Si; green=3coordinated $\mathrm{Si}$; red $=\mathrm{BO}$; magenta $=\mathrm{NBO}$; turquoise $=$ free $\mathrm{O}$; purple $=\mathrm{P}$; blue $=\mathrm{Ca}$ ). 


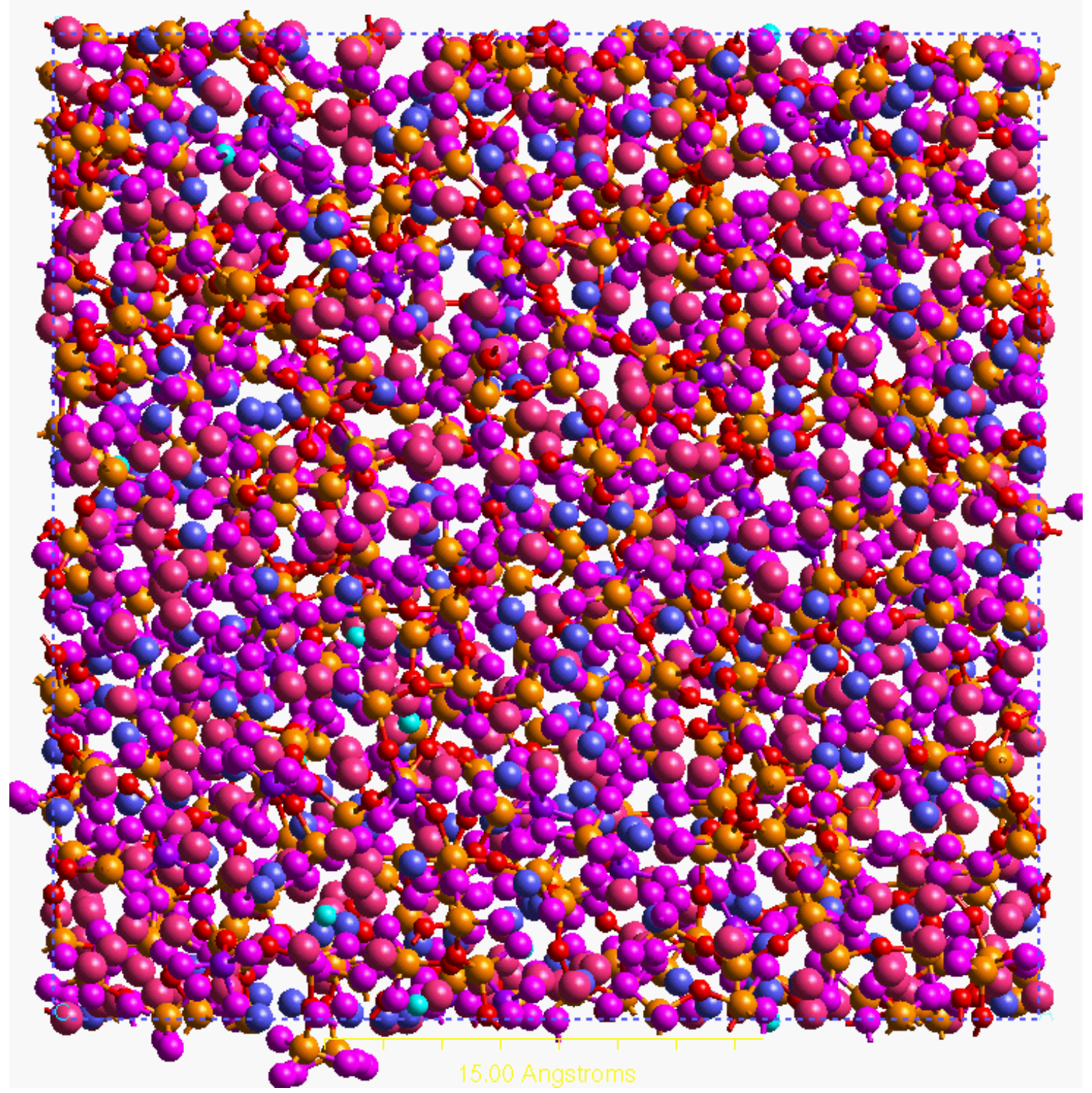

Figure 4. Bulk structure of 45S5 (2835-atoms) glass (yellow=4-coordinated Si; green=3coordinated $\mathrm{Si}$; red $=\mathrm{BO}$; magenta $=\mathrm{NBO}$; turquoise $=$ free $\mathrm{O}$; purple $=\mathrm{P}$; blue $=\mathrm{Ca}$ ). 


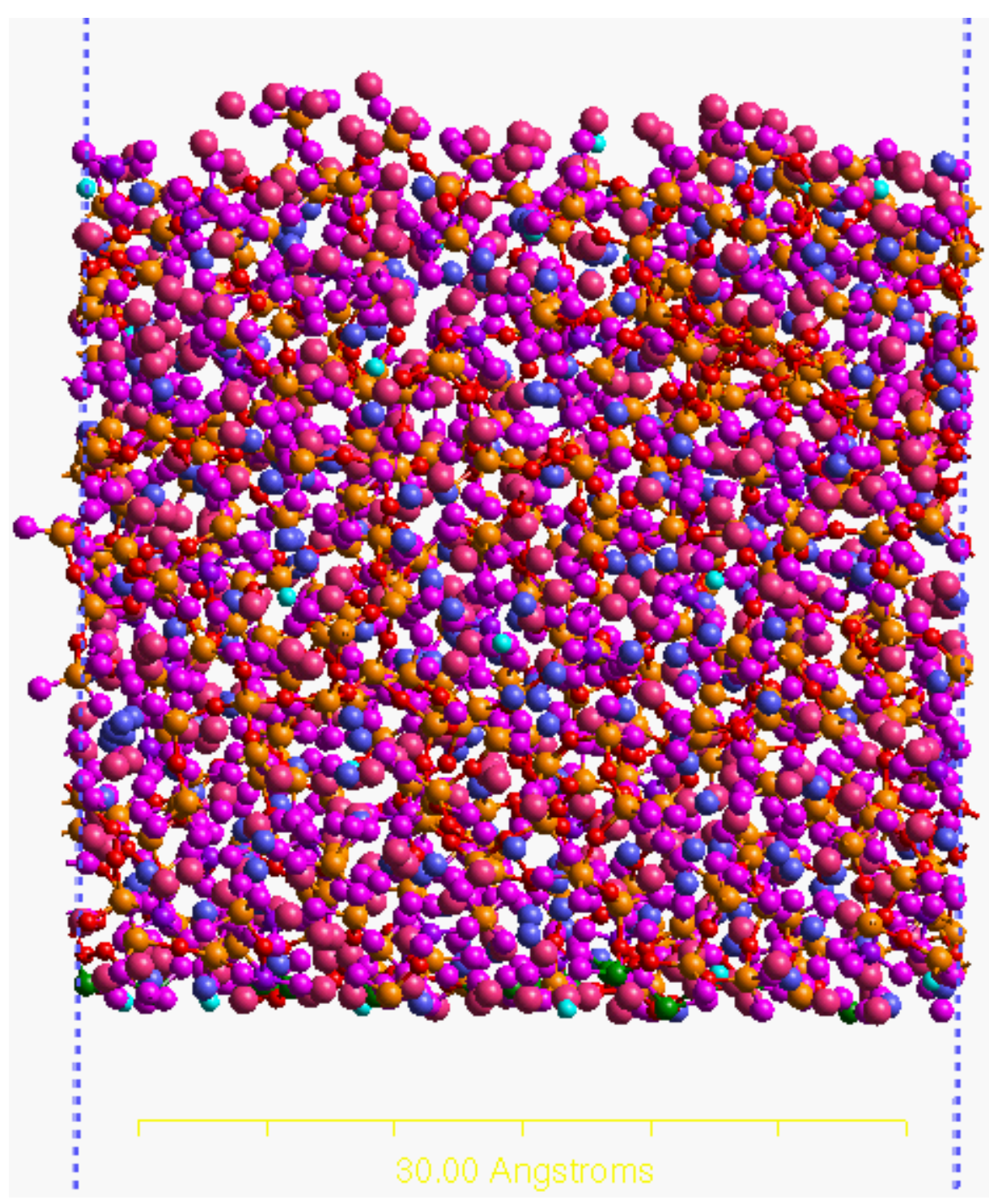

Figure 5. Surface structure of 45S5 (2835-atoms) glass (yellow=4-coordinated Si; green $=3$-coordinated $\mathrm{Si}$; red $=\mathrm{BO}$; magenta $=\mathrm{NBO}$; turquoise $=$ free $\mathrm{O}$; purple $=\mathrm{P}$; blue $=\mathrm{Ca}$ ). 


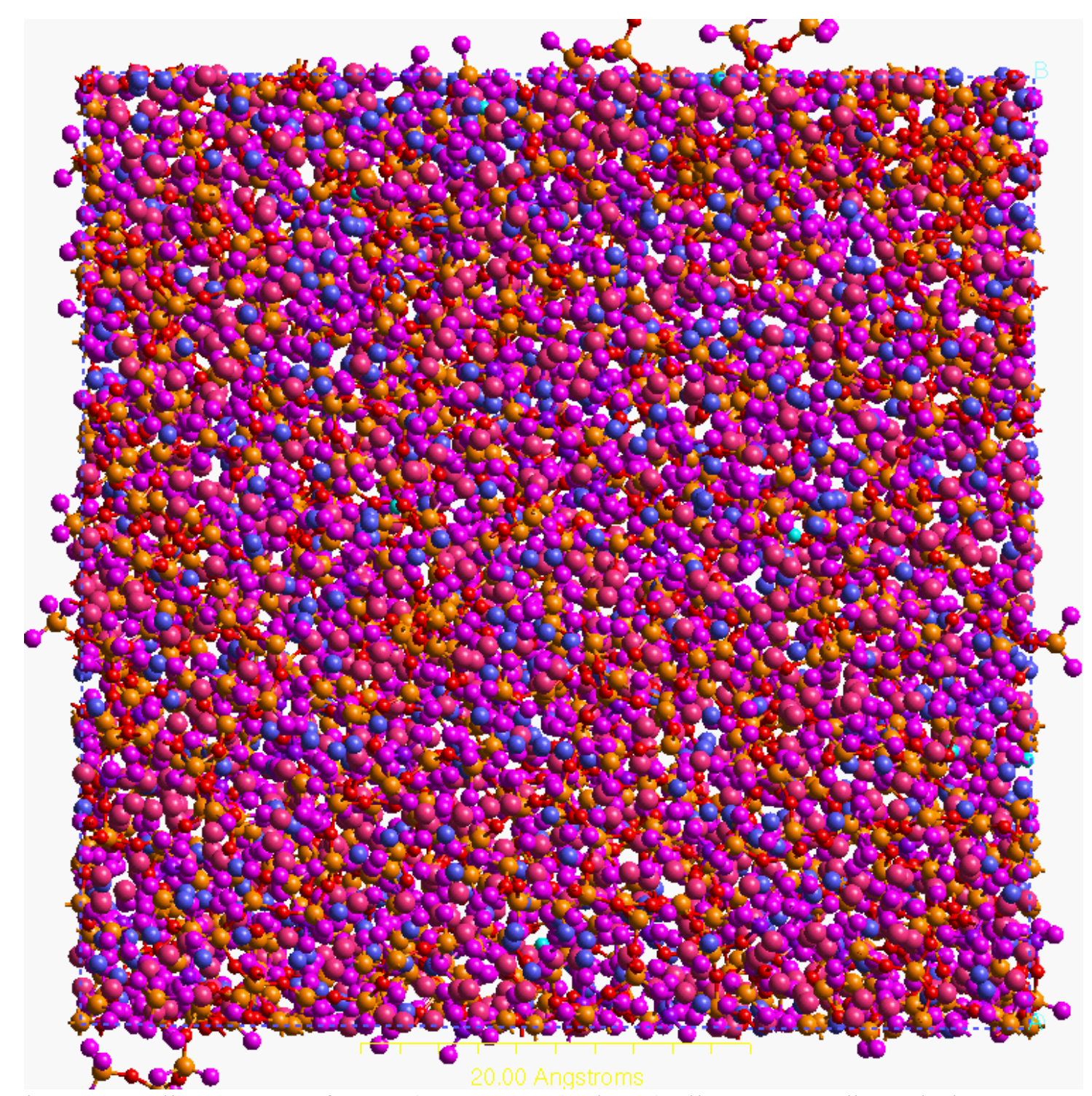

Figure 6. Bulk structure of 45S5 (8505-atoms) glass (yellow=4-coordinated Si; green=3coordinated $\mathrm{Si}$; red $=\mathrm{BO}$; magenta $=\mathrm{NBO}$; turquoise $=$ free $\mathrm{O}$; purple $=\mathrm{P}$; blue $=\mathrm{Ca}$ ).

\section{Qn distribution}

Table III. shows the $\mathrm{Q}_{\mathrm{n}}$ distribution for $\mathrm{Si}$ and $\mathrm{P}$ in the four simulations (where $n$ is the number of bridging oxygen on the network former). The $\mathrm{Si} \mathrm{Q}_{\mathrm{n}}$ distribution seems relatively consistent among the simulations, with the exception of no $\mathrm{Q}_{0}$ groups present in the two smallest simulations. Also, in the 376-atom simulation, there seems to be an increase in the number of $\mathrm{Q}_{2}$ groups and decrease in number of $\mathrm{Q}_{3}$ groups.

With only two and eight $\mathrm{P}$ atoms in the 94 - and 376-atom simulations, respectively, there is understandably not much resolution in their $\mathrm{Q}_{\mathrm{n}}$ distributions. However, there seems to be fairly good agreement between the two largest simulations 
and the two smaller simulations are not drastically different. It is possible that with multiple starting configurations, the $\mathrm{Q}_{\mathrm{n}}$ distributions could average out to numbers closer to those of the larger simulations.

Table III. $\mathrm{Q}_{\mathrm{n}}$ distributions (percentage) for $\mathrm{Si}$ and $\mathrm{P}$ for three simulation sizes.

\begin{tabular}{|c|c|c|c|c|}
\hline $\mathbf{n}$ & $\mathbf{8 5 0 5 - S i}$ & $\mathbf{2 8 3 5 - S i}$ & $\mathbf{3 7 6 - S i}$ & $\mathbf{9 4 - S i}$ \\
\hline 0 & 4.3 & 3.7 & 0.0 & 0.0 \\
\hline 1 & 21.3 & 19.1 & 20.0 & 26.7 \\
\hline 2 & 42.3 & 46.0 & 60.0 & 46.7 \\
\hline 3 & 26.5 & 26.9 & 16.7 & 20.0 \\
\hline 4 & 5.6 & 4.3 & 3.3 & 6.7 \\
\hline
\end{tabular}

\begin{tabular}{|c|c|c|c|c|}
\hline $\mathbf{n}$ & $\mathbf{8 5 0 5 - P}$ & $\mathbf{2 8 3 5 - P}$ & 376-P & 94-P \\
\hline 0 & 57.1 & 53.8 & 37.5 & 50.0 \\
\hline 1 & 33.3 & 38.5 & 50.0 & 50.0 \\
\hline 2 & 9.6 & 7.7 & 12.5 & 0.0 \\
\hline 3 & 0.0 & 0.0 & 0.0 & 0.0 \\
\hline 4 & 0.0 & 0.0 & 0.0 & 0.0 \\
\hline
\end{tabular}

\section{Oxygen states}

The relative amount of each state of oxygen is fairly consistent among the simulations (Table IV.). One noticeable difference is the presence of free oxygen in the two largest simulations.

Table IV. Oxygen states (percentage) for three simulation sizes.

\begin{tabular}{|c|c|c|c|}
\hline \#atoms & BO & NBO & freeO \\
\hline 8505 & 31.5 & 68.1 & 0.4 \\
\hline 2835 & 31.7 & 67.7 & 0.6 \\
\hline 376 & 30.8 & 69.2 & 0.0 \\
\hline 94 & 30.8 & 69.2 & 0.0 \\
\hline
\end{tabular}

\section{TDF}

Overall, the Si-O TDF agree fairly well, including the peak around $4 \AA$, for all simulation sizes to about $6 \AA$ where the 94 -atom simulation deviates due to the simulation box size (Fig. 7). A close-up of the first peak shows that there is some deviation, likely due to the small sample size, in the 94-atom simulation.

The P-O TDF shows quite a bit more scatter among the three smallest simulations, even in the first peak, though the two largest simulations lay nearly on top of each other even at large separations (Fig. 8). This is at least in part attributed to the smaller number of $\mathrm{P}$ atoms as compared to $\mathrm{Si}$ atoms. 

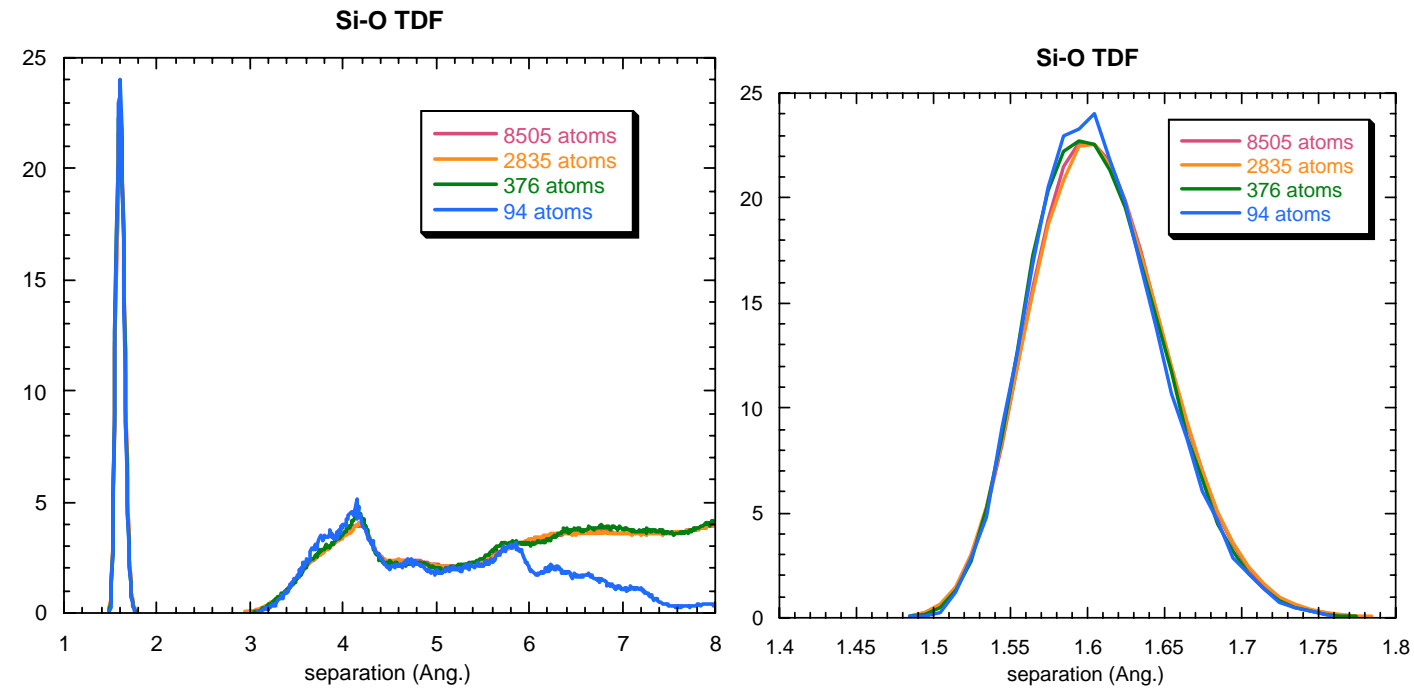

Figure 7. Si-O TDF (first peak) for 3 sizes of simulation.
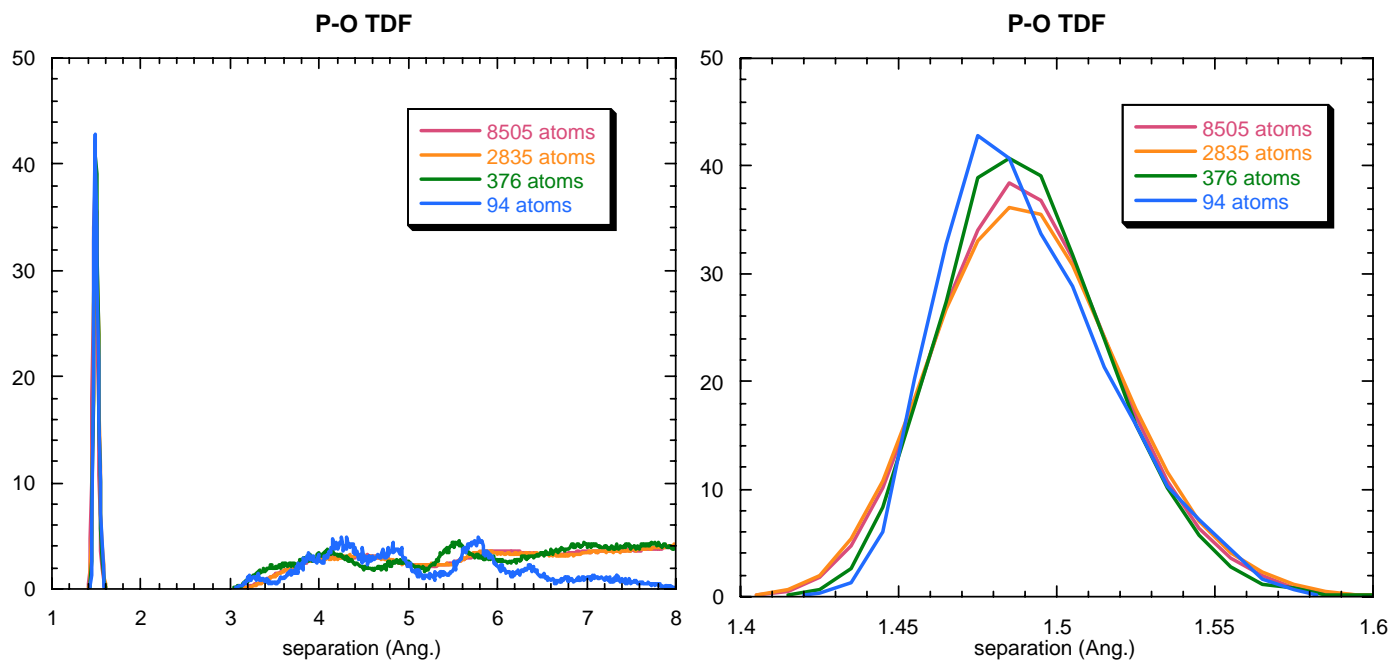

Figure 8. P-O TDF (first peak) for 3 sizes of simulation.

Another interesting TDF is the P-P TDF (Fig. 9). There is a discrepancy among all four simulations, even the two largest. This may be attributed also in part to the small number of $\mathrm{P}$ atoms, but the difference between the 2835- and 8505-atom simulations is still quite striking. It appears that even at 2835 atoms, the P-P TDF may not be a good representation of an actual bulk sample. The single peak of the 94-atom simulation comes about because it contains only $2 \mathrm{P}$ atoms. It is unclear why there is a large peak in the 376-atom simulation at around $4 \AA$ that is not present in any of the other simulations. This may show some effect of the particular configuration and may be averaged out by using multiple starting configurations. 


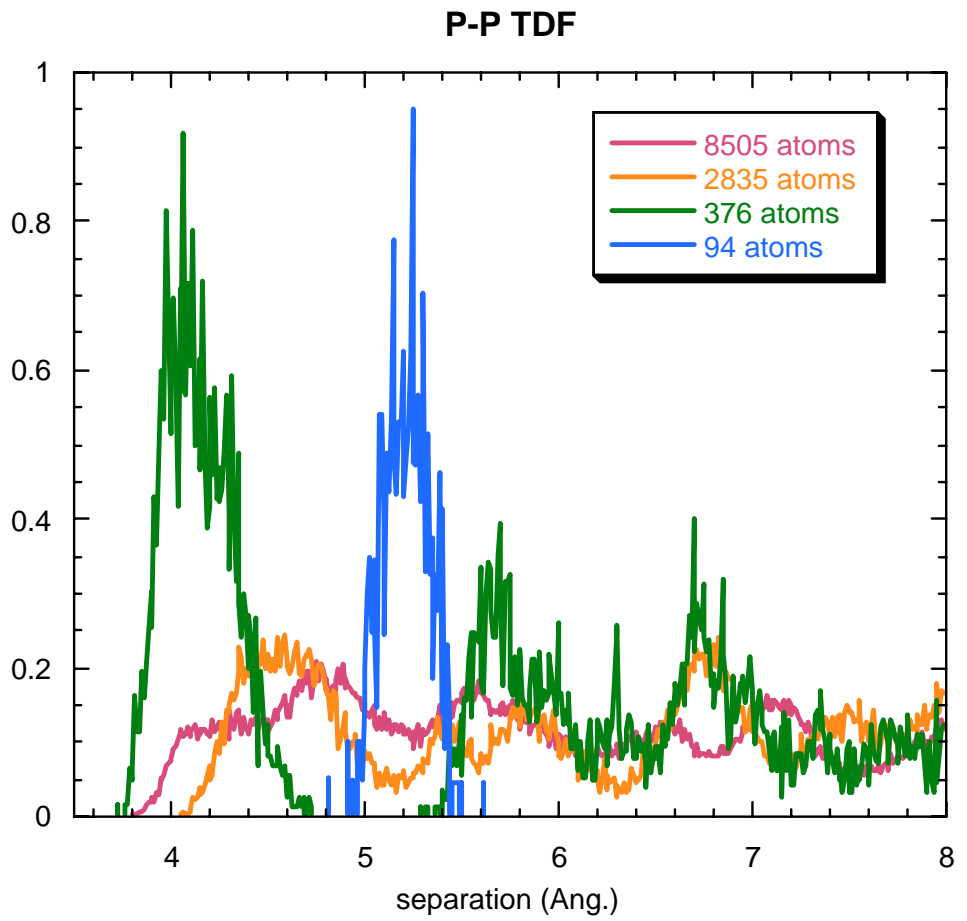

Figure 9. P-P TDF for 3 sizes of simulation.

\section{BAD}

The O-Si-O and O-P-O BAD seem to be centered at about the same points, but with much scatter for the two smallest simulations (Fig. 10). The O-P-O shows more overall scatter, again due to the fewer number of $\mathrm{P}$ atoms present. There is a small difference in the O-P-O peak of even the two largest simulations.
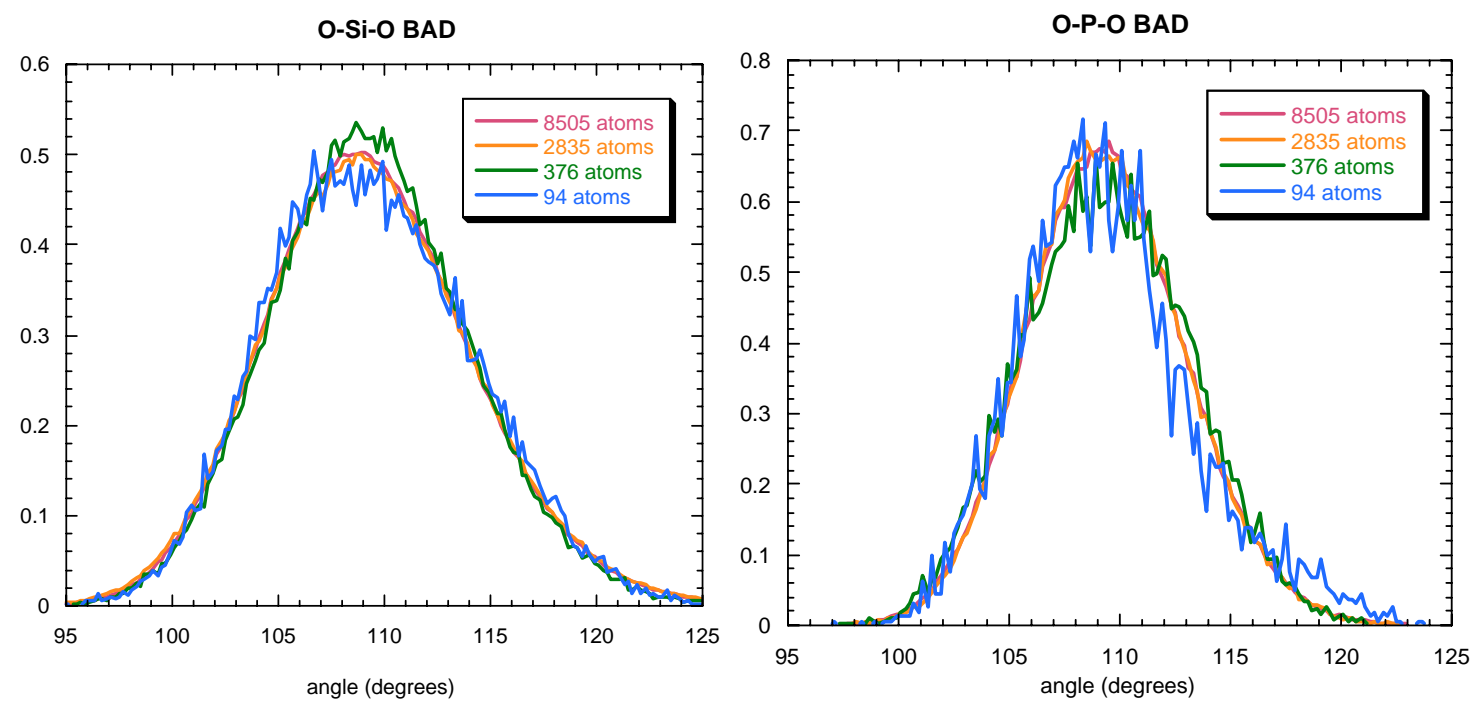

Figure 10. O-Si-O and O-P-O BAD for 3 sizes of simulation.

ZDP

The ZDP (atomic density profile perpendicular to surface) of the 2835- and 94atom simulations are shown in Figure 11. It should be noted that the 94 -atom simulation 
had only the lower $25 \%$ of its box frozen while the 2835 -atom simulation had $50 \%$ frozen. One of the obvious differences between the two ZDP is the seemingly erratic nature of the overall density in the 94-atom simulation. The major contributor to this seems to be the influence of the BO component to the overall density. It is not clear that there is a natural explanation for this, and it may be another case of the sample-size effect, though there is a small increase in the BO density near the surface in the 2835atom simulation.

Figure 12 shows the same data, this time normalized to the total density at each point. While the 2835-atom simulation shows that an increased proportion of the $\mathrm{Na}$ atoms are at the surface, the 94-atom simulation shows an increase in $\mathrm{Ca}$ atoms. Again, this may be averaged out over multiple simulations.
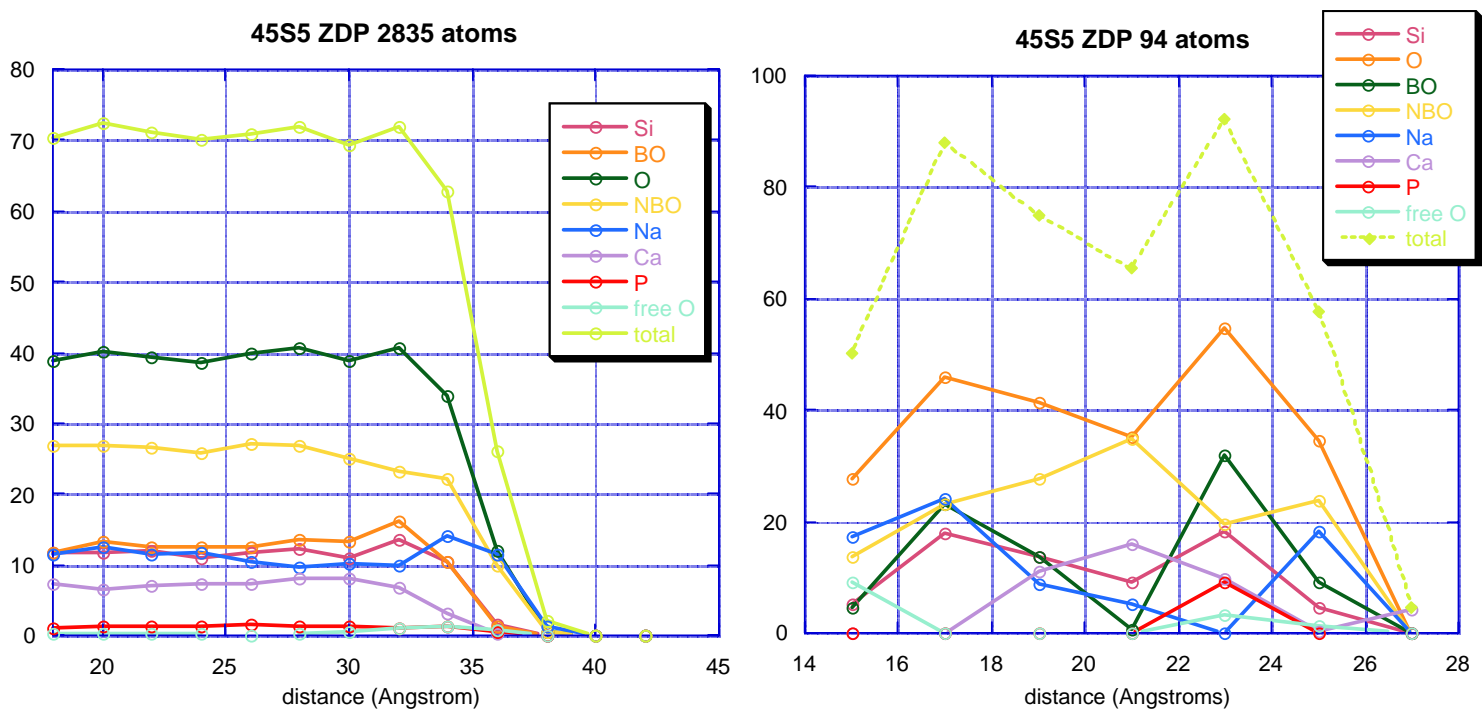

Figure 11. ZDP for 2835 and 94 -atom simulations (\#atoms $/ \mathrm{nm}^{3}$ ).
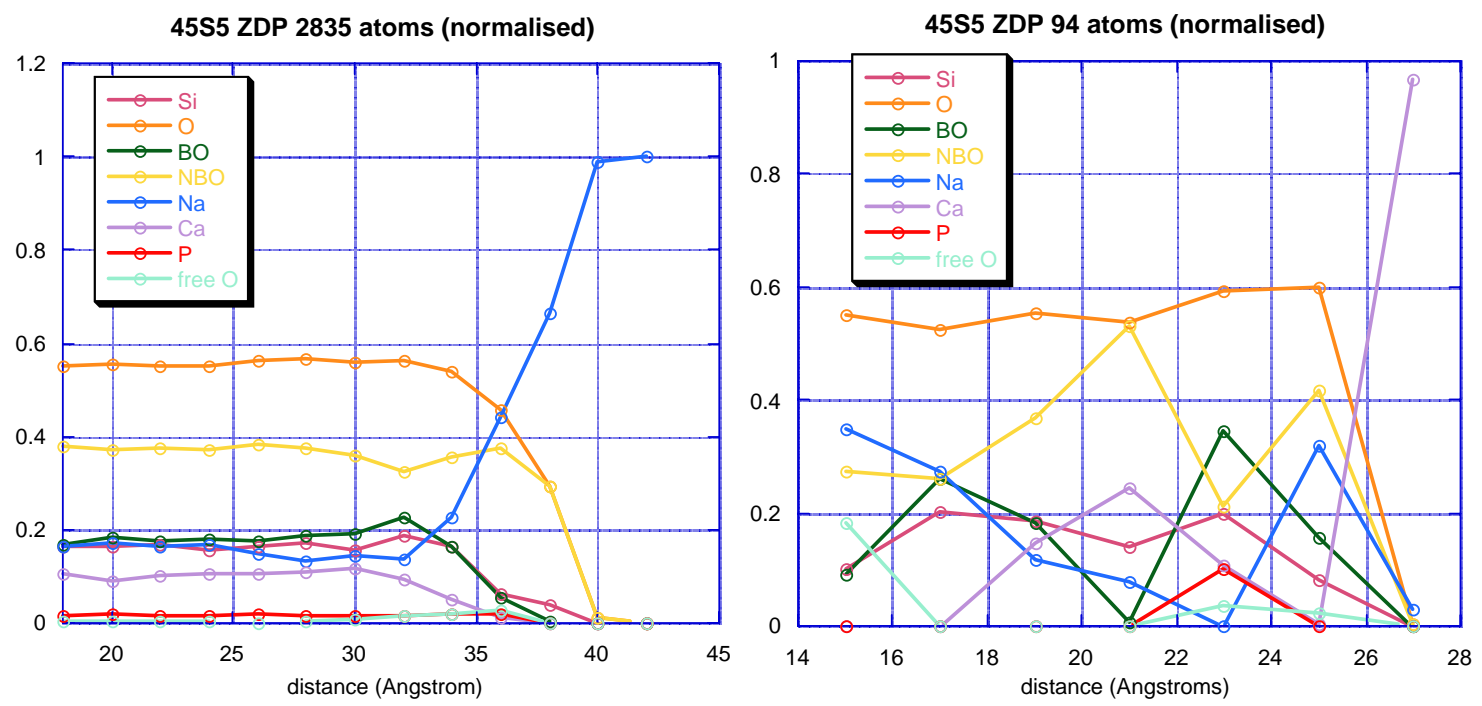
Figure 12. ZDP for 2835 and 94-atom simulations (normalised to total density).

\section{Network Fragmentation}

One issue with regard to small simulation size that is particular to this multicomponent glass is that of the size of bulk network fragments (continuous pieces of the network composed of $\mathrm{O}, \mathrm{P}$, and $\mathrm{Si}$ in the bulk simulation). In the 2835-atom simulation, it was noted that the network structure is greatly fragmented, as compared to a pure silica glass which is continuous throughout the entire simulation. This fragmentation is expected to aide in disintegration of the glass in vivo; indeed, of three compositions studied (at around 3000 atoms each), the more fragmented structures corresponded to a greater bioactivity of the glass in vivo.

For the 2835-atom 45S5 simulation, network fragments on the order of 25-60 atoms were seen, though $80 \%$ of the network atoms were contained in one large fragment (Table V). This $80 \%$ figure is fairly consistent among the three smallest simulations, though the largest simulation has only about $70 \%$ of network atoms in the largest fragment. This is a little troubling as it indicates a fundamental influence of simulation size on the overall bulk structure of the glass. It is also worth noting that the largest fragment in the 94-atom simulation is on the order of a smaller fragment in the 2835atom simulation and that the second and third largest fragments of the 8505-atom simulation are larger than the entire 94-atom simulation. This is important when considering the effect of simulation size on what is actually simulated; is the smaller simulation indicative of the bulk or a simulation of a smaller fragment of the larger simulation?

Table V. shows the sizes of network fragments (left) in each of the simulations and the fraction (right) of the total number of network atoms that fragment represents. The 21-, 17-, 13-, and 9-atom fragments for the 8505-atom simulation are consolidated into one entry each. For all simulations, the 5-atom $\mathrm{PO}_{4}$ (purple boxes) and $\mathrm{SiO}_{4}$ (yellow boxes) groups are also consolidated in Table V. The second largest fragment in the 94atom simulation is much larger fraction than that of any other simulation. The proportion of $\mathrm{PO}_{4}$ groups seems fairly consistent among all simulations. The proportion of $\mathrm{SiO}_{4}$ groups in the two largest simulations is consistent though there are no $\mathrm{SiO}_{4}$ groups present in the two smallest simulations. 
Table V. Network fragments by number of atoms (left) and that fragment's fraction of the total network atoms in the bulk simulation; 5-atom $\mathrm{PO}_{4}$ groups and $\mathrm{SiO}_{4}$ groups are consolidated in the purple and yellow boxes, respectively. The four boxes preceding the purple box for the 8505 simulation indicated the number of $21-, 17-, 13-$, and 9 -atom fragments.

\begin{tabular}{|c|c|c|c|}
\hline 8505-atom & 2835-atom & 376-atom & 94-atom \\
\hline 4455 & 1668 & 226 & 52 \\
\hline 109 & 57 & 17 & 13 \\
\hline 104 & 40 & 9 & $1 * 5=5$ \\
\hline 72 & 25 & 9 & 0 \\
\hline 65 & 25 & $3 * 5=15$ & \\
\hline 57 & 9 & 0 & \\
\hline 41 & 9 & & \\
\hline 41 & 9 & & \\
\hline 29 & 9 & & \\
\hline 25 & 9 & & \\
\hline 24 & $28 * 5=140$ & & \\
\hline $5 * 21=105$ & $17 * 5=85$ & & \\
\hline $5 * 17=85$ & & & \\
\hline $10 * 13=130$ & & & \\
\hline $18 * 9=162$ & & & \\
\hline $89 * 5=445$ & & & \\
\hline $60 * 5=300$ & & & \\
\hline
\end{tabular}

\begin{tabular}{|c|c|c|c|}
\hline 8505-atom & 2835-atom & 376-atom & 94-atom \\
\hline 71.4 & 80.6 & 81.9 & 75.4 \\
\hline 1.7 & 2.8 & 6.2 & 18.8 \\
\hline 1.7 & 1.9 & 3.3 & 7.2 \\
\hline 1.2 & 1.2 & 3.3 & 0.0 \\
\hline 1.0 & 1.2 & 5.4 & \\
\hline 0.9 & 0.4 & 0.0 & \\
\hline 0.7 & 0.4 & & \\
\hline 0.7 & 0.4 & & \\
\hline 0.5 & 0.4 & & \\
\hline 0.4 & 0.4 & & \\
\hline 0.4 & 6.8 & & \\
\hline 1.7 & 4.1 & & \\
\hline 1.4 & & & \\
\hline 2.1 & & & \\
\hline 2.6 & & & \\
\hline 7.1 & & & \\
\hline 4.8 & & & \\
\hline
\end{tabular}

\section{Conclusion}

The project this summer began as a study to get a better description of the energetics associated with the interaction of water with the surface of a bioactive glass. A few unanticipated roadblocks presented themselves with the use of a 94-atom simulation box in creating both bulk and surface multicomponent glasses. While the final structures seem stable, they were created using a different technique, so the resulting structures demanded further scrutiny. By comparing them with larger simulations, many differences have become evident.

While these final 94-atom structures can be used with NWCHEM to do some DFT calculations, the results must be put in the perspective of these structural differences noticed in both the bulk and surface structures. Clearly, a simulation-size effect has shown itself in a glass in which network fragmentation has been related to the glass' function, so these issues should be cleared up when considering the results of the DFT calculations and the suitability of small simulations for multicomponent glasses.

\section{Comments on PNNL's Interfacial and Condensed Phase Summer Research Institute}

Though the project did not proceed 'smoothly' throughout the summer, I still have the opportunity to remotely use MPP2 to run DFT calculations. The institute gave me a great opportunity to experience research in a national laboratory setting. It gave me access to software (NWCHEM) and computing power (MPP2) that I would not otherwise have been able to use in my project, and without which a DFT study would be impossible 
to undertake at my current institution. I was able to get a better perspective on the project I have been working on as my thesis project in Alfred. I think this report reflects that; though most of the results presented here were not gotten at PNNL, the results I did get helped me significantly get a better understanding of the project as it stood before my participation in the institute.

Most of all, the expertise of the staff was invaluable in my learning about density functional theory and molecular dynamics (a technique I have been using for four years). Particularly helpful during my stay in Richland were René Corrales, Jincheng Du, Shawn Kathmann, Jun Li, and Danny Chang. They enriched my experience through their conversation, both in and out of the lab. 


\section{Attachement A \\ Interfacial and Condensed Phase Chemical Physics Summer Research Institute (ICPCPSRI): follow-up Questionnaire}

Dear Colleague;

From my perspective, the inaugural year of PNNL's ICPCPSRI went well. However, your perspectives are much more important and will largely determine the fate of this program. Therefore, I encourage each of you to take a few minutes to answer some questions and to append any additional comments, suggestions, etc. to your reply. I would appreciate reply by 16 September, even if it is incomplete. If at a later time you wish to add anything, please do so.

\section{Directions}

Not every question applies to everyone, and it is not necessary to answer everything that does apply. The more complete your answers, the more useful they are. I may wish to use your comments in follow-on documentation, please indicate whether you wish for me to attribute the comments to you or if you would prefer to remain anonymous.

- May I quote you with attribution?

\section{Section I. Contact Information}

Please provide me with current contact information, your name address, e-mail, phone, etc.

Are you willing to be contacted for further follow-up questions?

\section{Section II. Measures of Success (or not)}

\section{PNNL mentors and faculty advisors}

1. Was this summer's activity part of an on-going collaboration?

2. How likely is this summer's experience to contribute to future collaboration outside the auspices of the summer program?

3. Did the work contribute to your research or project aims? How?

All

4. How likely is this work to appear in publication(s), presentation(s)? (If you are planning to publish or present, please provide some detail—where, when, etc.approximately.) 
5. (Grad students only) How likely is this work to contribute to your Ph.D. Thesis?

6. Were any new avenues of research opened?

7. Did your work here produce any intellectual property (IP) or is it likely to lead to IP development? (If yes, please give some description without disclosing the IP itself.)

8. Would you recommend others to apply to or participate in this program?

Please add any additional comments you wish on the intellectual, technical or scientific success or shortcomings of the program.

Section III. The Experience

All

1. What aspects of the program did you feel were particularly good?

2. What can we do to improve the program?

3. (Participants who visited PNNL) What difficulties did you encounter coming here or during your stay?

\section{Section IV. Comments}

Please take a moment to add any additional comments, opinions or anecdotes about the program, your visit, the research, etc.

Thank you,

Stephan E. Barlow

Director

ICPCPSRI

(509) 376-9051

se.barlow@pnl.gov

PS: The name of this program and even the alphabet soup "ICPCPSRI" is quite cumbersome; I welcome suggestions for a better name. (SEB) 

Stephan E. Barlow Director ICPCPSRI (509) 376-9051

se.barlow@pnl.gov 